\title{
Elucidation of protein interactions in complex samples by protein-protein cross-linking of synaptosomes
}

\author{
Dissertation \\ for the award of the degree \\ "Doctor rerum naturalium" \\ of the Georg-August-Universität Göttingen
}

within the doctoral program

"Biomolecules: Structure-Function- Dynamics"

of the Georg-August University School of Science (GAUSS)

\author{
submitted by \\ Iwan Parfentev \\ from Tallinn, Estonia
}

Göttingen, 2019 


\section{Thesis Committee}

Prof. Dr. Henning Urlaub, Bioanalytical M ass Spectrometry group, Max-Planck-Institute for Biophysical Chemistry

Prof. Dr. Reinhard Jahn, Department of Neurobiology, Max-Planck-Institute for Biophysical Chemistry

Prof. Dr. Silvio Rizzoli, Department of Neuro- and Sensory Physiology, University M edical Center Göttingen

\section{Members of the Examination Board}

Referee: Prof. Dr. Henning Urlaub, Bioanalytical Mass Spectrometry group, M ax-Planck-Institute for Biophysical Chemistry

$2^{\text {nd }}$ Referee: Prof. Dr. Reinhard Jahn, Department of Neurobiology, Max-Planck-Institute for Biophysical Chemistry

\section{Further members of the Examination Board}

Prof. Dr. Silvio Rizzoli, Department of Neuro- and Sensory Physiology, University M edical Center Göttingen

Dr. Juliane Liepe, Quantitative and Systems Biology group, M ax-Planck-Institute for Biophysical Chemistry

Dr. Vladimir Pena, M acromolecular crystallography Research Group, M ax-Planck-Institute for Biophysical Chemistry

Alexis C. Faesen, Ph.D., Biochemistry of Signal Dynamics, Max-Planck-Institute for Biophysical Chemistry 


Dedicated to 277 rats, who gave their brains for this thesis. 



\section{Abstract}

Synapses are essential structures for inter-cellular communication in the central nervous system between neuronal cells. They form highly-specialized compartments that convert electrical to chemical signals, i.e. neurotransmitter release by synaptic vesicle fusion. A highly dynamic network of interacting proteins facilitates the cycle of synaptic vesicle recruitment, docking, priming and $\mathrm{Ca}^{2+}$ triggered exocytosis. Subsequently, fused vesicles are retrieved by endocytosis and are prepared for another round of the cycle. Synaptosomes are pinched-off synaptic nerve terminals that can be prepared from neuronal tissue. Synaptosomes are model systems for synapse function, because they are physiologically active and can be stimulated to release neurotransmitters.

The goal of the present study was to identify and quantify the protein interaction dynamics present in the synapse. This was attempted by quantitative chemical cross-linking mass spectrometry (XL-MS) of synaptosomes in resting and excited state. XL-MS is an established method in structural biology that provides low resolution structural information about protein conformations and interactions. However, analysis of more complex samples like synaptosomes is challenging. The database search space exponentially increases with the number of theoretically cross-linkable peptides derived from a protein sequence database, which impairs sensitivity. Furthermore, synaptosome preparations frequently contain co-migrating myelin fragments and intra- and extrasynaptosomal mitochondria. Therefore, proteomic analyses of synaptosomes are dominated by proteins of mitochondrial and myelinic origin. This thesis has established a biochemical workflow to deplete contaminant proteins originating from myelin fragments and intrasynaptosomal mitochondria, thereby enabling a stronger focus on synaptic proteins. In addition, a peptide-focused database search approach for XL-M S was developed, which first identifies peptides that participate in a cross-linking reaction followed by providing these cross-linking candidates for database search. In contrast to considering all theoretically cross-linkable peptides derived from a proteome, the search space is much smaller resulting in a higher sensitivity. The novel approach was validated on purified cross-linked complexes of known structure and on in vivo cross-linked bacteria.

Combinging the peptide-focused database search approach with the improved protocol for synaptosome purification resulted in the creation of a cross-linking protein interaction network of resting and excited synaptosomes. Numerous known and novel protein interactions were identified involving, e.g. ion transporting ATPases, synapsins, 14-3-3 scaffold proteins, G-proteins and Stxbp1/M unc18-1. Furthermore, quantitative XL-M S allowed the quantification of significant changes in protein conformations and interactions upon stimulation of synaptosomes. Significantly changed cross-linked residues were observed in $\mathrm{Ca}^{2+}$ and $\mathrm{Ca}^{2+} /$ calmodulin-binding proteins, e.g. synaptotagmin, Anxa6, alpha spectrin, and Camkv. Ion channels like PM CA, $\mathrm{Na}^{+} / \mathrm{K}^{+}$ATPase, SERCA, I3PR and VDAC exhibited significantly changed cross-linked residues under excited conditions. The implied conformational changes agreed with the respective ion channel function. Moreover, previously unknown conformational changes were observed in this thesis, e.g. a major domain movement in I3PR that might turn the channel inactive, a possible monomerization of Cend1, and an enhanced interaction between Camkll and neutral ceramidase.

A quantitative XL-MS analysis of changing protein interactions in complex samples like stimulated synaptosomes was not attempted before. This thesis therefore analyzed the most complex and transiently changing system by quantitative XL-MS, to date. 


\section{Abbreviations}

\section{Abbreviation}

\section{$A B C$}

$\mathrm{ACN}$

Anxa6

Camkll

Camkv

CSM

CV

DDA

DIA

DSP

DSS

DTT

EGTA

ER

fC

GABA

I3PR

IAA

LB medium

$\mathrm{m} / \mathrm{z}$

OD

PBS

PMCA

Pol II

ppm

pSEC

rpm

SCX

SDS-PAGE

SERCA

Snap25

Stx1

Stxbp1/M unc18-1

Syt1

TFA

TFIIH

XL-MS
Ammonium bicarbonate

acetonitril

Annexin A6

calcium/calmodulin-dependent protein kinase II

CaM kinase-like vesicle-associated protein

Cross-link to spectrum match

Coefficient of variance

Data-dependent acquisition, (shotgun proteomics)

Data-independent acquisition

dithiobis(succinimidyl propionate)

Disuccinimidyl suberate

Dithiothreitol

Triethylene glycol diamine tetraacetic acid

endoplasmic reticulum

final concentration

gamma-aminobutyric acid

inositol-1,4,5-trisphosphate receptor

lodoacetamide

Lysogeny broth medium

mass to charge

Optical density

Phosphate buffered saline

Plasma membrane $\mathrm{Ca}^{2+}$-transporting ATPase

RNA polymerase II

parts per million

peptide size-exclusion chromatography

revolutions per minute

Strong cation exchange

Sodium dodecyl sulphate polyacrylamide gel electrophoresis

Sarcoendoplasmic reticulum $\mathrm{Ca}^{2+}$-transporting ATPase

synaptosomal-associated protein 25

Syntaxin-1

Syntaxin-binding protein 1, mammalian homolog of Unc18

Synaptotagmin-1

trifluoroacetic acid

Transcription factor IIH

Cross-linking mass spectrometry 


\section{List of figures}

Figure 1: Scheme of the main steps of the synaptic vesicle cycle and involved proteins. ..................10

Figure 2: Schematic representation of the peptide-focused cross-linking workflow.........................30

Figure 3: Comparison of conventional cross-linking with the peptide-focused approach on purified

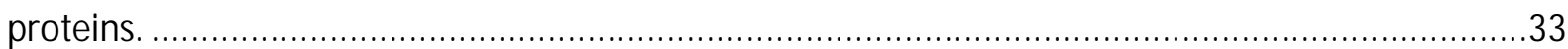

Figure 4: Comparison of DSS and DSP cross-linking without cleavage of cross-linker. ......................34

Figure 5: Interaction surfaces of EF-Tu on the ribosome. Bacteria were treated as described in chapter

2.2.3.

Figure 6: Scatter plot of fold changes of synaptosomes prepared with discontinuous Ficoll gradient and an additional continuous sucrose gradient plotted against the negative $\log _{10} p$-value (volcano plot)..

Figure 7: Proteomics label-free quantification analysis of synaptosomal plasma membrane preparations with a reported ${ }^{123}$ (A) and an adapted protocol (B).

Figure 8: Scatter plot of fold changes of synaptosomes prepared by Ficoll and continuous sucrose gradient in comparison to subsequent lysis and mitochondria depletion plotted against the negative $\log _{10}$ p-value (volcano plot).

Figure 9: Venn diagram comparing unique cross-links identified from differentially fractionated replicates of cross-linked synaptosomes..

Figure 10: Gain in unique peptides per replicate for the generation of a combined peptide database. Synaptosomes were cross-linked with thiol-cleavable DSP.

Figure 11: Distribution of proteins that are represented in the peptide database over the abundance range of the synaptosome proteome.

Figure 12: Cross-linking interaction map of identified synapse-related proteins.

Figure 13: Cross-linking interaction map of identified synaptic membrane receptors and channel proteins. See figure 12 .

Figure 14: Cross-linking interaction map of identified cell adhesion and cytoskeleton-related proteins. See figure 12

Figure 15: Scatter plot of significant fold-changes of unique cross-linked residues in synaptosomes under excited conditions in comparison to resting conditions plotted against the negative $\log _{10} p$-value.

Figure 16: Significantly changed cross-links displayed on different conformations of the sarcoendoplasmic $\mathrm{Ca}^{2+}$ transporting ATPase.

Figure 17: Significantly changed cross-links within plasma membrane $\mathrm{Ca}^{2+}$ transporting ATPase (PMCA).

Figure 18: Significantly changed cross-links for $\mathrm{Na}^{+} / \mathrm{K}^{+} \mathrm{ATPase}$.

Figure 19: Significantly changed cross-links within homotetrameric inositol-1,4,5-trisphosphate receptor (I3PR).

Figure 20: Significantly changed cross-links within calcium/calmodulin-dependent protein kinase II (Camkll).

Figure 21: Significantly changed cross-links within and between spectrin alpha, beta and ankyrin-2..

Figure 22: Significantly changed cross-links within synaptotagmin I (Syt1) ................................... 70

Figure 23: Significantly changed cross-links within annexin A6 (Anxa6).........................................71 
Figure 24: Significantly changed cross-links under excited conditions within cell cycle exit and neuronal

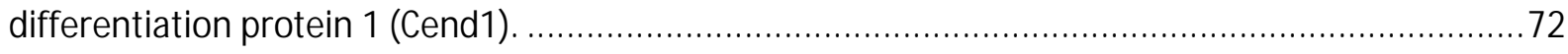

Figure 25: Significantly changed cross-links within voltage-dependent anion channel (VDAC) ..........74 Figure 26: Scatter plot of fold-changes of unique cross-link sites under excited conditions in comparison to resting conditions plotted against the negative $\log _{10} p$-value for three exemplary complexes that did

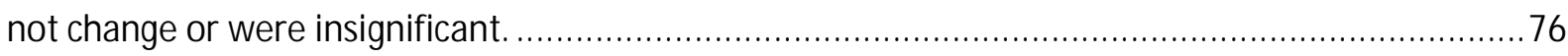




\section{List of tables}

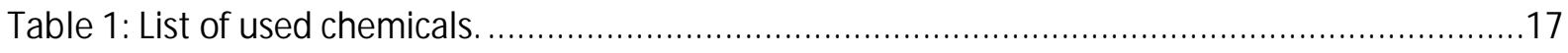

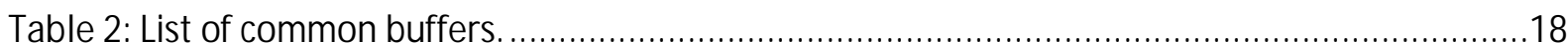

Table 3: List of consumables used in the study ..................................................................... 18

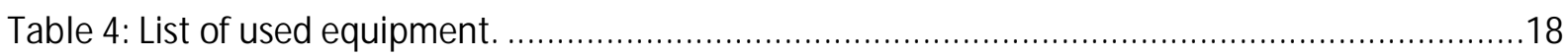

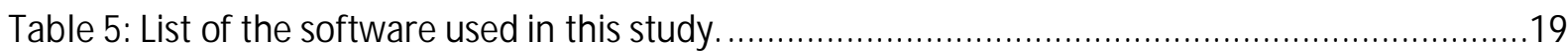

Table 6: Number of CSM s identified with the conventional and peptide-focused approach for purified

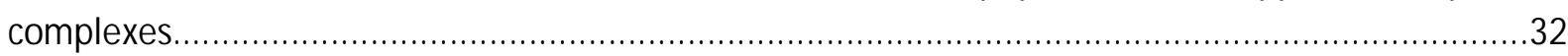

Table 7: Comparison of a conventional and peptide-focused database approach applied on Bacillus

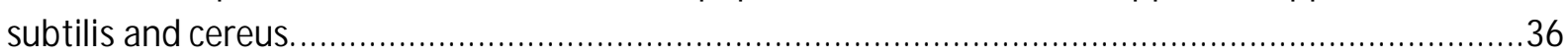

Table 8: Comparison of a conventional and peptide-focused database approach applied on rattus norvegicus.

Table 9: List of significantly changed unique cross-linked residues in synaptosomes upon stimulation (1.3 $\mathrm{mM} \mathrm{CaCl} 2,50 \mathrm{mM} \mathrm{KCl}$ ) in comparison to resting conditions ( $0.5 \mathrm{mM}$ EGTA, $50 \mathrm{mM} \mathrm{KCl})$. 


\section{Table of Contents}

Abstract

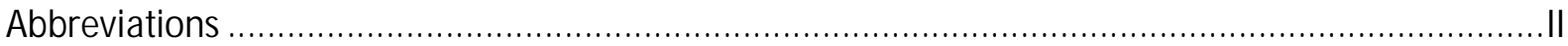

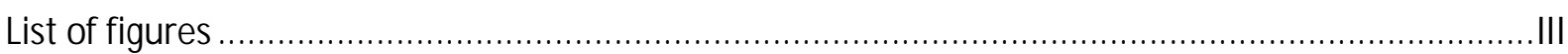

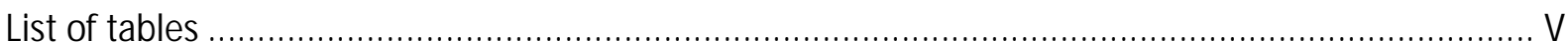

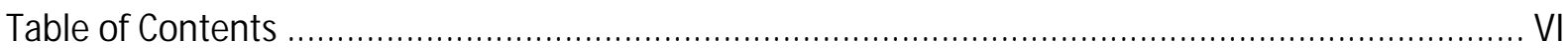

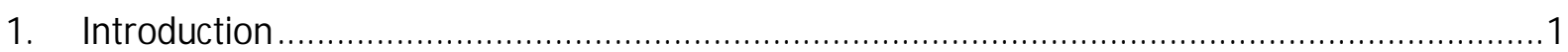

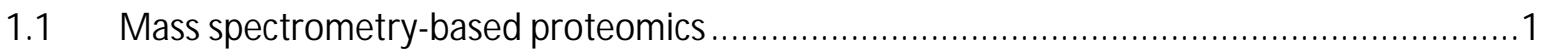

1.1.1 Principle of M S-based proteomics......................................................................

1.1.2 Protein identification by database searching ...................................................... 2

1.1.3 Quantitative mass spectrometry ....................................................................

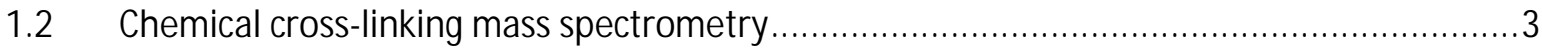

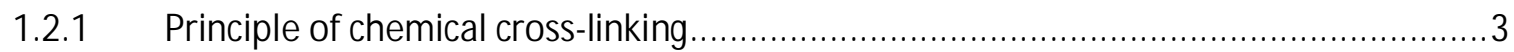

1.2.2 Identification of cross-linked peptides by mass spectrometry ...................................

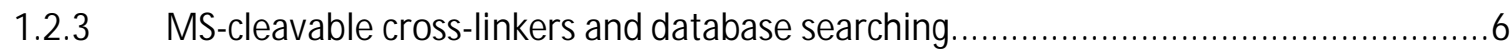

1.2.4 Quantitative cross-linking mass spectrometry .................................................

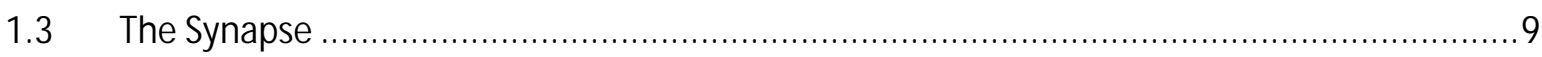

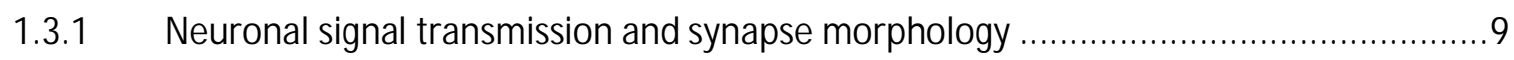

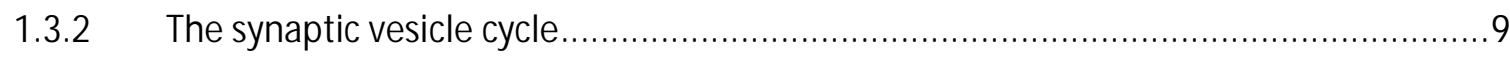

1.3.3 Protein composition of synaptic vesicles............................................................

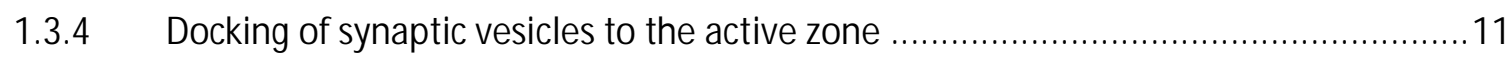

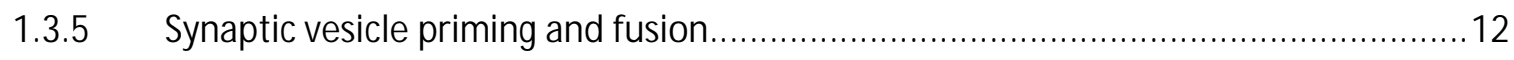

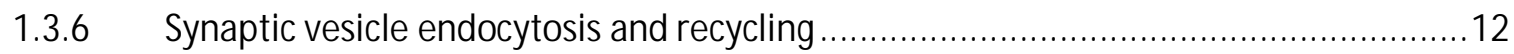

1.3.7 Signal transmission on the postsynaptic side ....................................................... 13

1.3.8 Synaptic cytoskeleton and cell adhesion proteins.................................................. 13

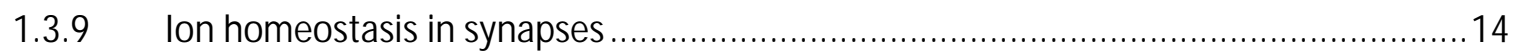

1.3.10 Synaptosomes as model systems for the study of synapses.................................15

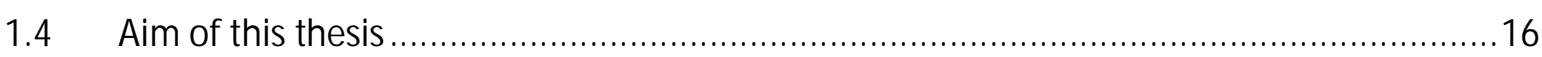

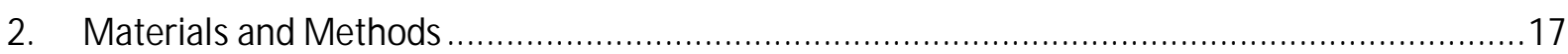

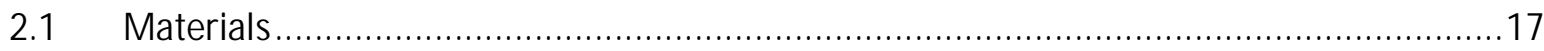

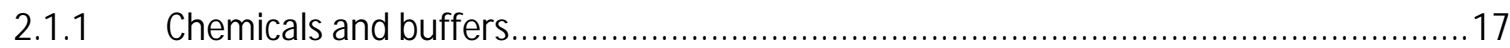

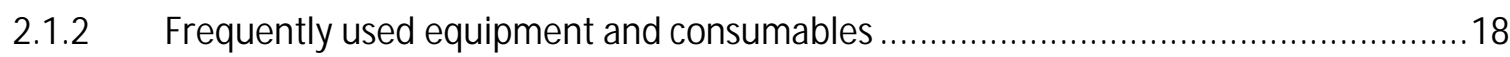

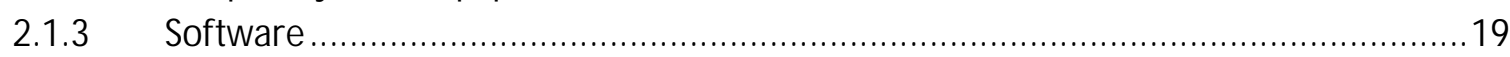

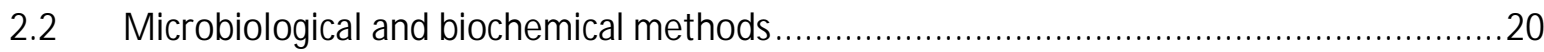

2.2.1 Purification and cross-linking of Pol II, TFIIH, and $20 \mathrm{~S}$ proteasome ...........................20

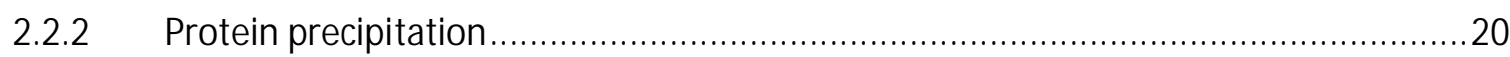

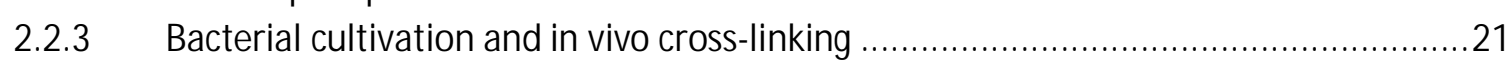

2.2.4 Synaptosome preparation, cross-linking and depletion of myelin fragments and

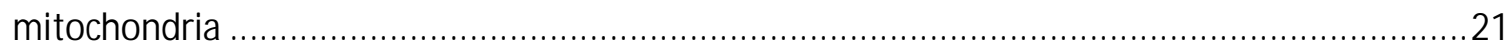

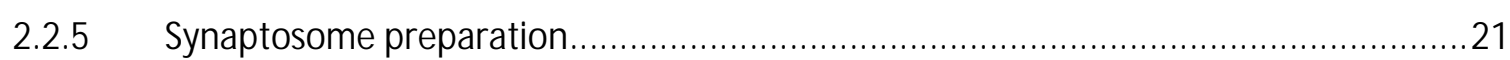

2.2.6 Depletion of myelin fragments by continuous sucrose gradient centrifugation ..........22

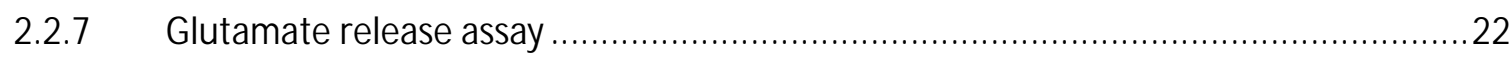




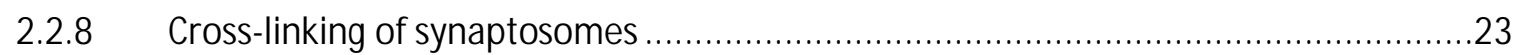

2.2.9 Depletion of mitochondria by discontinuous sucrose gradient centrifugation ............23

2.3 M ass Spectrometry and Proteomics methods ............................................................24

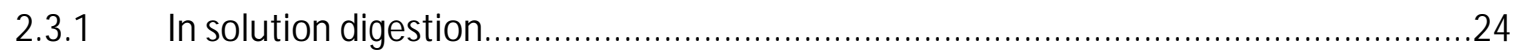

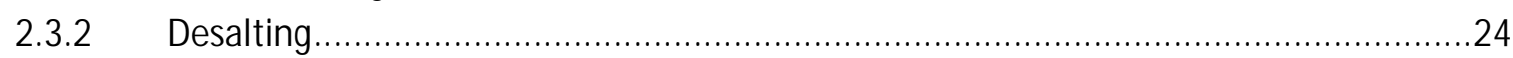

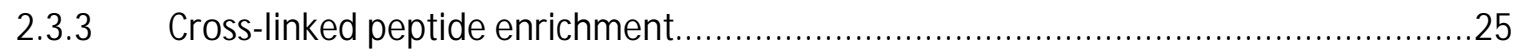

2.3.4 Enrichment by strong cation exchange chromatography ........................................25

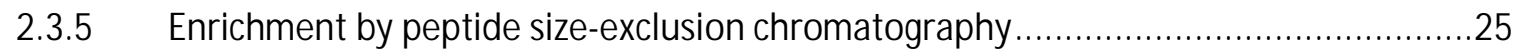

2.3.6 Cleavage of disulphide bridges in DSP-cross-linked samples .....................................25

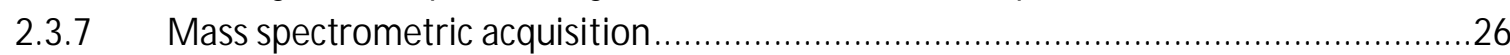

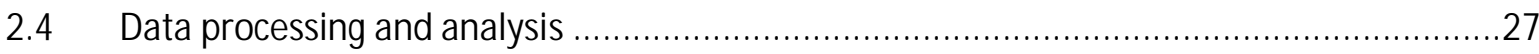

2.4.1 Proteomics label-free quantification analysis of myelin- and mitochondria-depleted

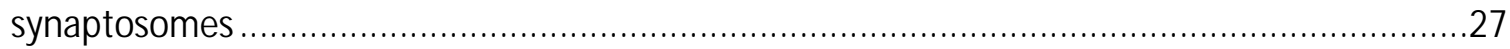

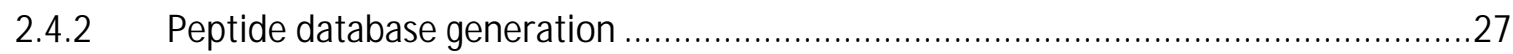

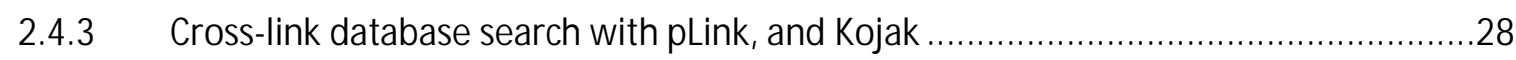

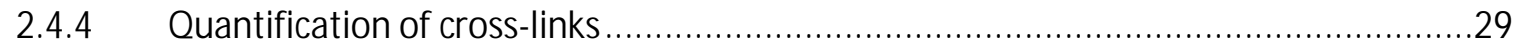

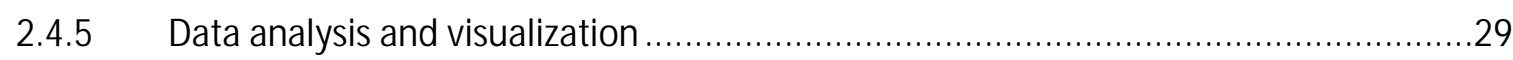

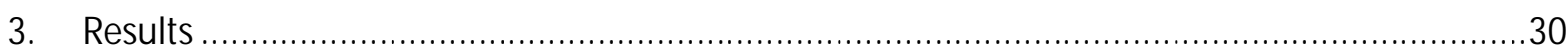

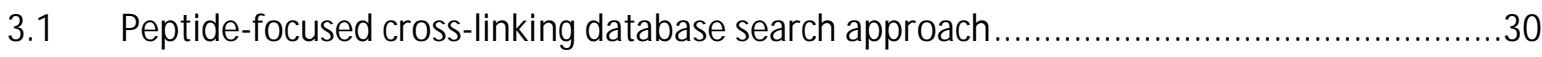

3.2 Application of the peptide-focused approach on purified complexes ................................31

3.3 Application of the peptide-focused approach on cross-linked bacteria..............................35

3.3.1 Performance comparison of the conventional and peptide-focused approach ............35

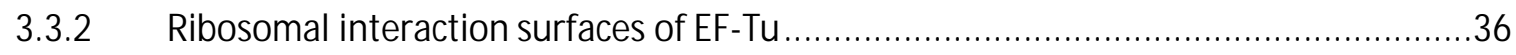

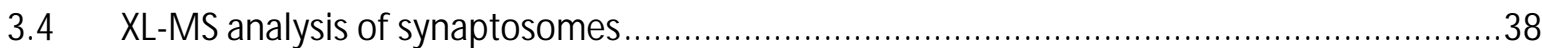

3.4.1 Modifications of the synaptosome preparation protocol.......................................38

3.4.2 Depletion of myelin fragments by continuous sucrose gradient centrifugation............38

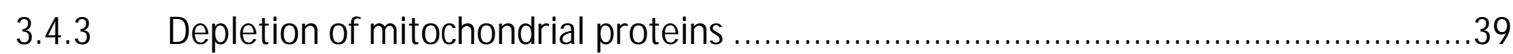

3.4.4 Strong cation exchange chromatography as an additional method for cross-linked

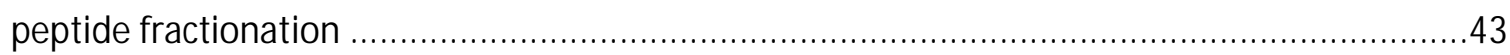

3.4.5 Application of a peptide-focused approach on rat brain synaptosomes......................44

3.4.6 The protein cross-linking interactome of rat brain synapses....................................47

3.4.7 Quantitative changes of cross-links in excited synaptosomes ...................................53

3.4.8 Significantly changed cross-links within ion-channels .............................................61

3.4.9 Significantly changed cross-links within calcium-binding proteins............................68

3.4.10 Significantly changed cross-links in proteins, which do not directly bind calcium ........72

3.4.11 Significantly changed cross-links within mitochondrial proteins ..............................73

3.4.12 Proteins with no significantly changed cross-links .................................................. 74

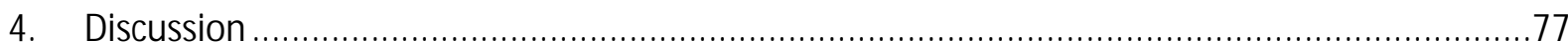

4.1 Advantages and disadvantages of applying a peptide-focused database search approach for XL-MS 77

4.1.1 Validation of the peptide-focused approach on purified cross-linked complexes........78

4.1.2 Generation of a peptide database ...................................................................... 
4.1.3 Comparison of the peptide-focused approach to alternative strategies for database

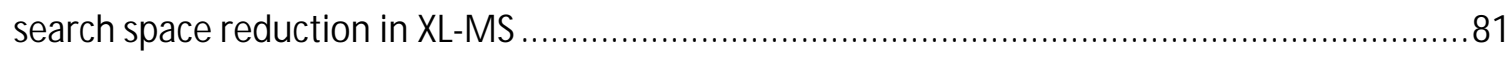

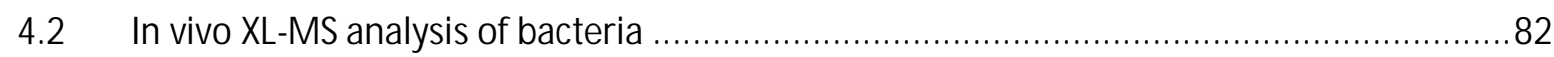

$4.3 \mathrm{XL}-\mathrm{MS}$ analysis of purified and functional synaptosomes ............................................ 83

4.3.1 Depletion of mitochondrial and myelin proteins for a stronger focus on synaptic proteins 83

4.3.2 The protein interaction network of functional synaptosomes captured by XL-MS ......85

4.3.3 Novel protein interactions captured by XL-MS .................................................. 87

4.4 Quantitative XL-M S applied on synaptosomes in resting and excited state .....................91

4.4.1 General considerations regarding quantitative XL-MS of stimulated synaptosomes ...92

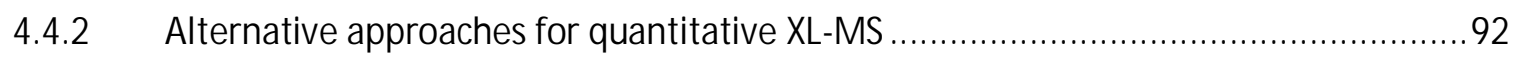

4.4.3 Proteins residing in intracellular $\mathrm{Ca}^{2+}$-storage organelles...................................... 94

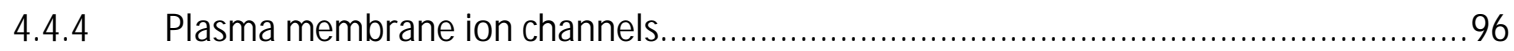

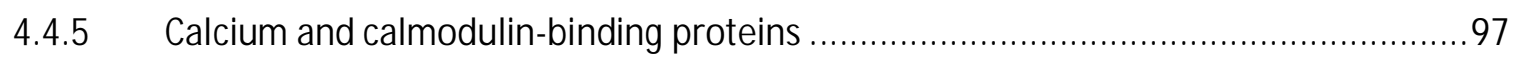

4.4.6 Cytoskeleton and proteins that do not bind calcium ............................................99

4.4.7 Synaptic protein complexes that did not display conformational changes upon

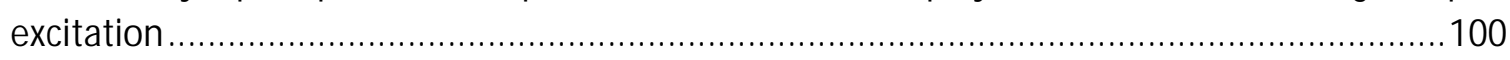

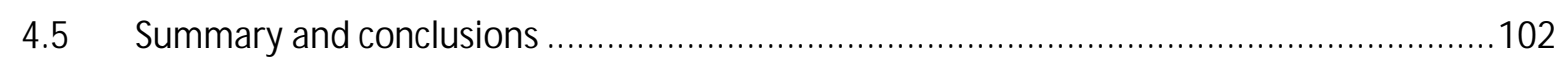

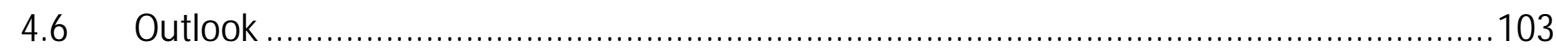

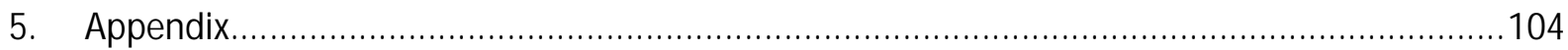

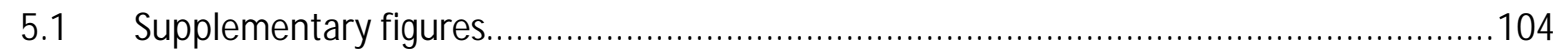

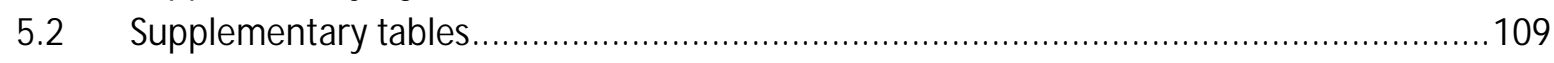

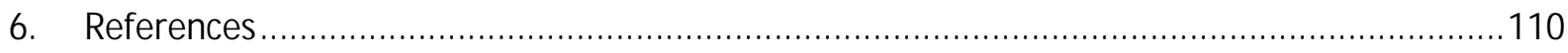

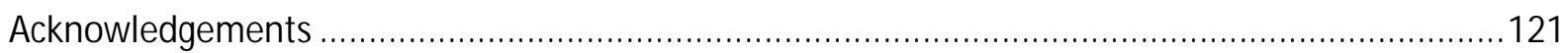

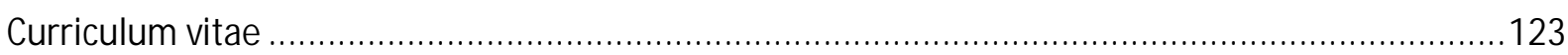




\section{Introduction}

\subsection{M ass spectrometry-based proteomics}

Mass spectrometry-based (MS) proteomics is a powerful tool to analyze most, if possible all, proteins in a sample at certain time points and under specific conditions ${ }^{2}$. This includes the identification and quantification of proteins, their post-translational modifications, interactions, structural conformations, and subcellular localizations. The plethora of information retrieved from distinct MS experiments holds the promise to advance our understanding of organisms and their physiology 3 .

\subsubsection{Principle of MS-based proteomics}

The basic principle of mass spectrometry is the determination of the mass to charge ratio $(\mathrm{m} / \mathrm{z})$ of ionized molecules in the vacuum. Although the precise measurement of $\mathrm{m} / \mathrm{z}$ of an intact protein is possible and helpful to determine distinct proteoforms of a protein ("top-down proteomics"), it is not suitable to analyze complex samples. Therefore, "bottom-up proteomics" has evolved as the state-ofthe-art technique for the identification of proteins and the analysis of complete proteomes. During a typical bottom-up experiment, proteins are cleaved to peptides with a specific protease, e.g. trypsin. Enzymatic digestion dramatically increases the number of analytes for MS experiments of a given protein sample. Mammalian proteomes consist of tens of thousands of unique proteins, which give rise to millions of peptides after protease digest. Therefore, the complexity of a sample has to be reduced prior to MS analysis. The peptide mixture is separated by a reversed-phase liquid chromatography that is coupled directly to the mass spectrometer (LC-MS) to reduce sample complexity ${ }^{3}$. Eluting peptides are ionized in an electrospray by solvent evaporation and attachment of charged ions, usually protons, to the peptide 4 . lonized peptides enter the mass spectrometer and are guided in a vacuum through the ion optics of the instrument to the mass analyzer. Examples for the latter are orbitraps $\mathrm{s}^{5}$ and quadrupoles, which separate ions according to their $\mathrm{m} / \mathrm{z}$ and detect them or guide them to a separate detector. Different types of mass analyzers are often combined in hybrid instruments like the $Q$ Exactive HF- $X$, which contains a quadrupole-orbitrap set-up ${ }^{6,7}$.

The instrument first records an M S1 spectrum of all analytes, so-called precursor ions, eluting from the $\mathrm{LC}$ at a specific time point. Subsequently, in a data-dependent acquisition (DDA) mode, also known as shotgun proteomics, the most abundant peptide ions are isolated and fragmented separately by collisions with an inert gas $\left(\mathrm{N}_{2}\right)$ and another spectrum is recorded. Peptides preferentially break along the peptide bond creating a so-called $\mathrm{N}$-terminal b-ion and a C-terminal y-ion series. The delta-masses between fragment ions correspond to amino acid masses. Therefore, fragment ion spectra, also called MS2 or MS/MS spectra, can be used to deduce the exact amino acid sequence of the peptide by de novo sequencing. The peptide sequence can in turn be mapped to the respective protein that was initially present in a sample? 


\subsubsection{Protein identification by database searching}

With the advent of the genomic era, peptide sequencing was replaced by matching experimental with theoretical fragment ion spectra derived from in silico digested proteins from sequence databases. This development facilitated the identification of thousands of proteins from a complex sample $e^{2,3}$. Early database search algorithms like Sequest assessed the similarity of experimental and theoretical spectra by cross-correlation analysis, which was efficient for low resolution data ${ }^{8}$. With the evolution of faster and more accurate MS instruments, new algorithms were developed, which can handle high resolution data. One example is the Andromeda algorithm integrated in the Maxquant software ${ }^{9}$. Similar to other database search software, Maxquant employs a probabilistic scoring model, which calculates the probability of $\mathrm{n}$ fragment ions from theoretical and experimental spectra matching by chance. Subsequently, a score is reported as the negative decadic logarithm of the probability of an identification being random ${ }^{10}$. Obviously, such a strategy features a certain degree of uncertainty whether an identified peptide is a true positive hit, especially for identifications with a low or moderate score. In addition to scoring, a target-decoy competition approach was therefore included to assess the number of false-positive identifications in an analysis. Acquired spectra are searched against the protein sequences of the species at hand as well as artificial decoy sequences that do not correspond to any known proteins and are generated by sequence reversal or shuffling. By definition, any match to a decoy peptide sequence is random and therefore enables an estimation of false-positive identifications in a dataset ${ }^{11}$. A false discovery rate (FDR) analysis after database search sorts all identifications according to score and applies a user-defined cut-off, usually $1 \%$, at spectral, peptide, or protein level.

The proteome of any organism is considered as complex. For example, protein concentrations in blood plasma were reported to have a dynamic range of eleven orders of magnitude ${ }^{12}$. In contrast to DNA and RNA, it is impossible to amplify proteins or peptides prior to analysis. Therefore, MS struggles to detect low abundant protein species in complex proteome samples. To reduce complexity, the sample is often prefractionated on protein and/or peptide level in addition to online reversed-phase LC. For example, proteins can be separated by their differences in molecular weight (gel electrophoresis, size exclusion), charge (strong cation/anion exchange), isoelectric point (off-gel electrophoresis, isoelectric focusing), or hydrophobicity (high $\mathrm{pH}$ reversed-phase) ${ }^{13-16}$.

\subsubsection{Quantitative mass spectrometry}

Undoubtedly, quantitative information is desirable in order to study alterations between two physiological states. The peptide ion intensity over the LC retention time detected by a mass spectrometer is linearly related to the peptide abundance at the exact same experimental conditions for the same peptide species. A label-free quantification option is included in many software suites such as $\mathrm{M}$ axquant ${ }^{17}$. However, label-free quantification is prone to variations occurring during sample processing, which accumulate to a non-systematic error that can distort quantification ${ }^{18}$. Stable isotope labelling was introduced to MS-based proteomics to overcome these limitations. Isotopic forms of a peptide have the same chemical properties and therefore behave near-identical during sample processing. Therefore, two samples that are tagged with different isotopic labels can be 
combined and processed together. Their isotopic mass difference separates the individual peptide signals during MS analysis allowing for a highly accurate relative quantification by comparing the respective signal intensities. Notew orthy, sample prefractionation does not influences quantification accuracy. Isotopic mass tags can be introduced metabolically, chemically, enzymatically, or are provided as a synthetic peptide standard. Metabolic stable isotope labelling by amino acids in cell culture (SILAC) ${ }^{19}$ enables the introduction of a mass tag at the earliest possible time point, i.e. during cultivation and cell growth. However, it is required that the used organism readily takes up and incorporates the respective amino acids from the medium and does not synthesize or metabolize them, as is often the case for the conversion of arginine to proline ${ }^{20}$.

Another possibility of isotopic tag incorporation is by the chemical derivatization of primary amine groups in peptides with, e.g. isobaric tandem mass tags (TMT). TMT consists of a reporter group containing the isotopic mass tag and a balance group, which renders the molecule isobaric. With this approach, peptides from different samples can be labelled with different TM T reagents and still appear as a single signal peak in MS1 scans. Only after fragmentation, the balance group is released as a neutral fragment and the signal peak of the reporter group provides quantitative information about the peptide and accordingly the respective protein in the sample ${ }^{21}$. On the one hand, TMT labels are universally applicable, allow multiplexing and do not increase the sample complexity. On the other hand, labelling and sample mixing is performed at a late stage, which renders quantification prone to variability occurring in earlier sample processing steps ${ }^{20}$.

All quantification methods mentioned above are based on DDA, which is characterized by low reproducibility. Because of the semi-stochastic precursor ion selection based on abundance, repeated LC-M S acquisitions of a sample always lead to a slightly different list of identifications. In contrast, no precursor ion selection is performed for data-independent acquisition (DIA). Several large consecutive $\mathrm{m} / \mathrm{z}$ windows covering the entire $\mathrm{m} / \mathrm{z}$ region are isolated and fragmented together enabling the acquisition of nearly all precursors and their respective fragment ions. Resulting complex chimeric fragment ion spectra are subsequently queried for the presence of peptide and fragment ion masses with assay libraries ${ }^{22}$. Due to the almost complete acquisition of ion signals, the number of missing values is low in comparison to data-dependent acquisition but necessitates the generation of spectral and assay libraries prior to quantification ${ }^{22}$.

Proteomic approaches are well suited to identify and quantify protein abundance changes in complex samples in response to environmental changes. However, the information about the protein threedimensional conformation and protein interactions is lost in bottom-up proteomics. Chemical crosslinking of proteins in conjunction with mass spectrometry has evolved as the method of choice to obtain structural information about single proteins and protein complexes ${ }^{23}$.

\subsection{Chemical cross-linking mass spectrometry}

\subsubsection{Principle of chemical cross-linking}

Chemical cross-linking of proteins was established in the 1970s for the analysis of oligomerization properties of histone proteins, ribosomes, and ATP synthases, among others ${ }^{24}$. In addition, it was used for the stabilization of large complexes like the ribosome for visualization with electron microscopy ${ }^{25}$. 
Chemical cross-linkers are molecules consisting of a spacer with two reactive groups on either end. When added to a protein sample, they react with functional groups on susceptible amino acid side chains to form a covalent link that bridges two amino acid residues in close proximity and within the distance restraint of the spacer ${ }^{23}$. Different reactivities are available, for example $\mathrm{N}$-hydroxy succinimide (NHS) esters targeting primary amines on lysine and at the protein N-terminus (e.g. disuccinimidyl suberate, DSS), maleimides targeting sulfhydryl groups on cysteines, UV-inducible diazirines reacting with any amino acid, and dihydrazides in combination with a coupling reagent targeting carboxyl groups on glutamate, aspartate and the protein C-terminus ${ }^{26,27}$. Cross-linkers can carry identical or different reactive groups, i.e. so-called homo- and heterobifunctional cross-linkers. Water-insoluble but membrane-permeable NHS-ester-based cross-linkers can contain a sulfonic acid residue on the NHS group that turns them water-soluble but membrane-impermeable. In addition, spacers of various kinds are available, i.e. different lengths, possibility of cleavage (e.g. thiol cleavable dithiobis[succinimidyl propionate], DSP), or a spacer with an affinity group for enrichment, so-called trifunctional cross-linkers ${ }^{26}$. Almost all aforementioned options can be freely combined to tailor the cross-linker towards the sample of interest.

After cross-linking and proteolytic digestion, the linkage between peptides can originate from two different proteins (inter-protein cross-link), from the same protein (intra-protein cross-link), within the same peptide (loop-link), or the cross-linker can react with one group only, while the other reactive group hydrolyses (dead-end link). However, the majority of theoretically reactive sites within a protein does not react with the cross-linker and gives rise to linear, i.e. not cross-linked, peptides ${ }^{28}$. Although this leads to a low yield in identifications of cross-linked peptides, a sub-stoichiometric cross-linking is desired for two reasons. First, the cross-linking reaction can lead to artificial, structural alterations of the protein that can already impair enzymatic activity ${ }^{29}$. For example, amide bonds created by reaction of NHS esters with primary amines lose their positive charge under physiological $\mathrm{pH}$, thereby disrupting electrostatic interactions ${ }^{26}$. Second, excessive cross-linking interferes with proteolytic digestion. This is especially true for NHS-based cross-linkers, since they target lysine residues that in turn cannot be cleaved by trypsin anymore ${ }^{30}$. In addition, excess cross-linker can generate aggregates involving more than two peptides.

Early use of the method involved cross-linking and selective cleavage in combination with gel electrophoresis and antibody detection of subunits ${ }^{24}$. With the emergence of M S-based proteomics, chemical cross-linking was combined with LC-MS to identify proteins forming oligomers in shifted gel bands ${ }^{31}$. However, an ideal combination of chemical cross-linking and mass spectrometry (XL-MS) has the capability to identify interacting proteins and the exact residues involved in a cross-link. Due to the covalent bond introduced, proteolytic digestion of cross-linked proteins yields cross-linked/ conjugated peptides that can be identified. XL-MS thereby provides low resolution structural information about spatially close amino acid residues in a protein or protein complex at the time of reaction ${ }^{28}$. This information proved to be a valuable supplement for other structural methods like cryogenic electron microscopy (cryo-EM). Reconstructed three-dimensional volumes of large protein complexes often have a low resolution, which does not allow for de novo modeling of protein subunits. Distance restraints obtained from XL-MS facilitate the exact localization of subunit structures within the density of the protein complex ${ }^{32}$. Such hybrid approaches of combining XL-M S and cryo-EM have led to the structural elucidation of spliceosomal complexes ${ }^{33}$ and the RNA polymerase transcription factor $F$ complex ${ }^{34}$, among others. 


\subsubsection{Identification of cross-linked peptides by mass spectrometry}

Analogous to the workflow outlined in chapter 1.1.1 and 1.1.2, cross-linked peptides can be analyzed and identified like non-cross-linked linear peptides. Due to the increased complexity of reaction products and the dominance of linear peptides, MS acquisition settings are often modified to preferentially target cross-linked peptides. For example, the LC gradient is adapted and singly and doubly charged precursor ions are excluded from fragmentation to favor more hydrophobic and multiply charged cross-linked peptides ${ }^{30}$. In comparison to linear peptides, M S2 spectra of cross-linked peptides are more complex. They contain linear fragment ions originating from the two peptides, fragment ions containing a peptide fragment, the cross-linker and the other intact linked peptide, as well as ions originating from multiple fragmentation events. Due to the higher complexity of MS2 spectra, a high mass resolution on MS2 level is important to avoid misassignments of fragment ions ${ }^{35}$. Theoretically, proteins in a database are in silico digested and all possible combinations of peptides need to be considered for database search of cross-linked peptides. This amounts to $\left(n^{2}+n\right) / 2$ possible pairs for $n$ peptides. This so-called " $n^{2}$ problem" describes that the database search space expands with the square of the number of peptide candidates in a database, thereby increasing computational costs and the risk of random matches ${ }^{23}$. For example, the number of theoretical cross-linked peptide candidates from 50 proteins already corresponds to the number of linear peptides derived from the human proteome ${ }^{36}$. XL-MS is often restricted to the analysis of samples of moderate size. More complex samples can be analyzed with prior knowledge of the most abundant proteins in a sample and usage of a limited database. The rationale behind this approach is the higher probability of abundant proteins to cross-link, the resulting cross-linked peptides to be selected for fragmentation, and that these cross-linked peptides yield a comprehensive fragmentation pattern ${ }^{37}$.

Early attempts for an automated analysis of cross-linked peptides involved the creation of a database of concatenated sequences of all possible peptide pairs and considering the cross-linker as a modification. This approach enabled the use of common search algorithms but had several drawbacks, e.g. scoring was not suited for cross-linked peptides and not all possible fragment ion types were considered $^{38,39}$. The first automated cross-linking search algorithm that could also handle large sequence databases was $x Q$ uest ${ }^{40}$. The algorithm initially depended on cross-linking of a protein sample with a mixture of isotopically labelled and non-labelled cross-linker, and fragmentation of both precursor ions arising from the isotopic shift. Spectra from the light and heavy cross-linked peptide pair are compared and fragment ions are sorted in common and uncommon ions. Uncommon ions derive from fragments that contain the isotopically labelled cross-linker. Common ions derive from linear fragments of the peptides and are used for a candidate peptide search based on ion indexing. This step facilitates database searching and enables the analysis of complex samples ${ }^{40}$. Another strategy realized by the Link $^{41}$ and $\mathrm{Kojak}^{42}$ software is similar to an error-tolerant database search. A database search of M S2 spectra is performed for linear peptides irrespective of the precursor mass, thereby considering the cross-linker and the other linked peptide as a variable modification. After a fast pre-scoring, peptide candidates are paired and checked whether the summed peptide masses plus cross-linker mass are equal to the precursor mass. Finally, fitting peptide pair candidates are fine scored and the best-scoring match is reported ${ }^{41,42}$. In addition, an expectation value is calculated by pLink, i.e. the probability of the match being random ${ }^{41}$. Target-decoy competition and FDR analysis can be performed for cross-linked peptides analogous to the FDR analysis of linear peptides and is often included in the database search software. However, due to peptide pairing in XL-MS database search, target peptides can be identified with a link to decoy peptides. The decoy database search space is 
therefore three times larger, i.e. combinations of decoy-decoy, target-decoy, and decoy-target peptides, which complicates FDR calculation ${ }^{41,43}$.

Because of probabilistic scoring and FDR calculation, a small portion of identified cross-links will be false positive assignments. The same is true for MS-based proteomics, however, it is far more critical in XL-MS, because every misassigned cross-link can lead to a wrong structural model or falsely reported protein interactions. Reasons for a misassignment include isobaric cross-linked peptides and an incomplete fragmentation pattern of one or both of the peptides in a cross-link pair ${ }^{44}$. To avoid false positives, spectral identifications can be manually inspected for qualitative attributes, e.g. a minimum number of identified fragment ions per peptide, the number of unassigned peaks in the spectrum, and spectral quality in terms of signal-to-noise ratio ${ }^{44}$. However, manual inspection of spectra is highly subjective and not feasible for large scale experiments yielding tens of thousands of spectra. Another strategy to evaluate the confidence of obtained identifications is to map cross-links onto available model structures of proteins present in the sample and measure the $\mathrm{C} \alpha-\mathrm{C} \alpha$ atom distance of crosslinked residues. The length of a fully extended DSS spacer is $11.4 \AA$. Adding the length of two lysine side chains, the cross-linker can therefore span the distance of approximately $24 \AA$. Due to intrinsic protein flexibility, a cut-off of 26 to $30 \AA$ was determined from molecular dynamics simulations that can account for the majority of observed lysine $\mathrm{C} \alpha-\mathrm{C} \alpha$ atom distances ${ }^{45}$. Cross-links are mapped onto available model structures and an individual score cut-off can be applied to control the number of cross-linked residues that exceed a $\mathrm{C} \alpha-\mathrm{C} \alpha$ atom distance of $30 \AA^{46}$. However, protein structures are rigid models of a protein and do not account for possible domain movements upon, e.g. ligand binding. Since all cross-links are reported equivalently, mapping them onto a model structure of one particular conformational state can lead to the observation of overlength links that actually arise from another conformational state. Therefore, overlength links can also indicate conformational flexibility and have to be evaluated individually for each protein model structure.

The above-mentioned database search algorithms have found an elegant way to address the $n^{2}$ problem and thereby enable the analysis of more complex samples like cross-linked bacteria, and even eukaryotic proteomes ${ }^{40,41}$. However, these studies identified only a few cross-links when applied on large database sizes. It was hypothesized that due to the large database search space, high-scoring decoy matches accumulate leading to a loss in sensitivity ${ }^{47}$. Due to the observed limitations, XL-MS analysis of complex samples is still performed with databases limited to the most abundant proteins of a sample ${ }^{37}$.

\subsubsection{MS-cleavable cross-linkers and database searching}

M S-cleavable cross-linkers were reported in the past, but the development of disuccinimidyl sulfoxide (DSSO) by Kao and colleagues has pioneered the usage of MS-cleavable cross-linkers for identification of protein cross-links in complex samples ${ }^{48,49}$. DSSO is an NHS-ester based cross-linker with a fourcarbon spacer and a central sulfoxide group. During fragmentation in MS2, the cross-linker breaks between the sulfoxide group and neighboring carbon atoms and releases the two peptides, one with a short fragment of the cross-linker and the other with a long one containing the sulfoxide group. Subsequently, the sulfoxide group hydrolyzes leading to a delta mass corresponding to sulfur between the two characteristic reporter ion pairs. These reporter ion pairs enable the calculation of the linear peptide mass, which facilitates database searching. By selecting the cleaved peptide ions in M S2 for 
an MS3 fragmentation event, linear peptides can be identified independently from their respective cross-linked peptide pair ${ }^{48,} 49$. This converts the $n^{2}$ problem of XL-MS to a $2 n$ problem thereby facilitating whole-proteome cross-linking and analysis. Liu and colleagues have developed a database search software called XlinkX, which can analyze data obtained from different fragmentation methods. For example, a cross-linked peptide precursor is first fragmented with collision-induced dissociation, which generates the mentioned reporter ion pairs. In addition, a fragmentation event with electron transfer dissociation ${ }^{50}$ is triggered on the same precursor that preferentially leads to peptide backbone fragmentations (so-called c- and z-ions) that provide sequence information for database search ${ }^{49,51}$. Furthermore, XlinkX 2.0 incorporated the analysis of M S3 spectra that can be triggered, if reporter ion peaks with a delta mass are present in the MS2 spectrum ${ }^{49}, 51$. The usage of MS-cleavable DSSO in combination with XlinkX enabled the analysis of complex samples like HeLa cells ${ }^{49}, 51$, mitochondria ${ }^{52}$ and nuclei ${ }^{53}$. However, sequential fragmentation of the same cross-linked peptide precursor with different methods requires more acquisition time, which reduces the number of precursors that are analyzed during an LC-M S run, thereby decreasing sensitivity.

\subsubsection{Quantitative cross-linking mass spectrometry}

Structural biology methods like x-ray crystallography and cryo-EM generate rigid models of a protein. However, proteins are flexible structures that include side chain rotations, peptide backbone dynamics and major domain movements. Protein flexibility is essential for protein function regarding cellular processes and protein interactions. As mentioned above, cross-links can therefore appear as overlength, if plotted on a structural model of a particular state. A change in protein conformation or complex composition also changes the positioning of lysines. Accordingly, the probability that particular lysines can cross-link with each other changes as well, which is displayed by the presence or absence of a particular cross-link or changed cross-link abundances between two states. Therefore, quantitative XL-MS is an important method to study protein dynamics in solution ${ }^{54}$.

Analogous to quantitative proteomics methods described in chapter 1.1.3, various quantitative methods exist to quantify cross-linked peptides. For example, cross-linkers can be used to introduce an isotopic label by cross-linking a protein in two functional states with a light and heavy cross-linker such as DSS-d0 and DSS-d4. The latter contains four deuterium atoms in the carbon spacer. After crosslinking of both protein states individually, samples are pooled, processed and analyzed together. Crosslinked peptide precursors are distinguishable in MS1 by a nominal delta mass of 4 Da originating from the label and can be used for relative quantification of precursor ion intensities. One of the first applications of this method was the quantification of the conformational change of a chloroplast Ftype ATPase in response to dephosphorylation ${ }^{55}$. Schmidt and colleagues ${ }^{55}$ extracted ion intensities over the retention time manually, which was tedious and not feasible for more complex samples. Fischer and colleagues ${ }^{1}$ published a program called $\mathrm{XiQ}$, which automated the extraction of ion intensities over the retention time. Isotope-labelled cross-link quantification was subsequently incorporated in the Maxquant software to benefit from its advantages regarding chromatographic feature detection and mass recalibration ${ }^{56}$.

Müller and colleagues ${ }^{57,58}$ have developed two approaches for label-free quantification by direct comparison of precursor ion intensities between different experiments. The first approach is based on DDA and was benchmarked for cross-linked bovine serum albumin ${ }^{57}$. The second is a workflow for the 
generation of assay libraries of cross-linked peptides for DIA label-free quantification. The sample was again benchmarked with cross-linked albumin alone and with a background of tryptic E. coli lysate. Reproducibility was very high for single albumin, but the complex peptide background deteriorated quantification reproducibility of cross-linked peptides significantly. Apparently, samples that are more complex than a single protein or protein complex represent a challenge for label-free quantification of cross-linked peptides ${ }^{58}$.

Yu and colleagues ${ }^{59}$ have developed a TMT-based isotopic labelling approach in combination with DSSO cross-linking. The method was benchmarked on a relatively simple sample, i.e. bovine heart cytochrome $c^{59}$. Thus, its feasibility on more complex cross-linking samples that necessitate extensive prefractionation is yet to be demonstrated.

The only quantitative cross-linking analyses on a proteome-wide scale were performed by Chavez and colleagues ${ }^{60,61}$. They cultivated HeLa cells with isotopically labelled lysine and arginine according to the SILAC method described in chapter 1.1.3. The differentially labelled cells were either treated by a permanent stimulus, i.e. resistance to chemotherapeutics and a chaperone inhibitor, or were untreated as a control, followed by sample pooling and cross-linking. Subsequent M Sanalysis enabled the quantification of altered protein abundances as well as cross-linked peptides, which are a result of changes in protein conformations and interactions in response to the stimulus ${ }^{60,61}$.

Quantitative XL-M S has become a valuable tool to study protein conformational dynamics in solution, although sample complexity is still a challenge ${ }^{54}$. The method is now applicable on more complex samples to study changing protein interactions and conformations in organelles and cells in response to a stimulus, e.g. in neuronal synapses. 


\subsection{The Synapse}

\subsubsection{Neuronal signal transmission and synapse morphology}

Neurons are electrically excitable cells that are characterized by a negative membrane potential under non-stimulated conditions, called resting potential. It is established by concentration gradients of sodium, potassium, chloride, and calcium across the membrane, which are maintained by energydependent ion pumps. Changes in membrane potential are sensed by voltage-dependent ion channels that control the ion flux across the membrane and thereby perpetuate or counteract the altered membrane potential. The neuron integrates changing membrane potentials and triggers an action potential if a particular threshold is exceeded, which is propagated along the axon to target cell $5^{62}$. Synapses are essential structures for inter-cellular communication in the central nervous system between neuronal cells. They form distinctive compartments consisting of a presynapse at axonal endings, a postsynapse on dendrites of a target cell and a synaptic cleft in between. To accomplish their task of signal transmission, synapses are highly-specialized morphologically and in terms of protein and lipid composition. When an action potential arrives at the synapse, the electrical signal is

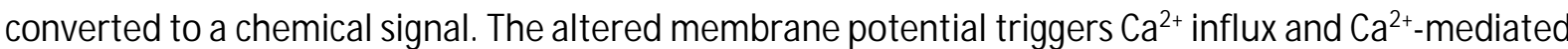
exocytosis of synaptic vesicles and the release of neurotransmitters into the synaptic cleft. The latter diffuse across the cleft to the post-synapse and bind to specific receptors. Neurotransmitter binding to postsynaptic receptors leads to an opening of an ion channel and subsequent depolarization and eventually the generation of an action potential and downstream signaling in the target cell ${ }^{63,64}$ Several types of synapses and neuronal cells exist that employ different neurotransmitters, e.g. excitatory synapses using glutamate, which is the most abundant type, inhibitory synapses using gamma-aminobutyric acid (GABA), and others ${ }^{65}$.

Early morphological characterizations of synapses were performed by electron microscopy ${ }^{66}$. The presynapse is filled with endosomes, a large number of synaptic vesicles ${ }^{67}$, and mitochondria that supply the energy needed for maintaining ion gradients and for processes involved in signal transmission ${ }^{68}$. The endoplasmic reticulum (ER) also forms long filaments that pervade from the neuron cell body into dendrites, axons and synapses ${ }^{69-71}$. Synaptic vesicles are scattered throughout the presynapse and a subset is docked along the membrane opposite the postsynapse. The postsynaptic membrane exhibits electron dense material in electron microscopic pictures after staining with heavy metals, which was therefore named postsynaptic density. The presynaptic side displays a less dense band called the active $z o n e^{66}$.

\subsubsection{The synaptic vesicle cycle}

Synapses have a finite pool of synaptic vesicles. After the arrival of an action potential and $\mathrm{Ca}^{2+}$ mediated exocytosis, synaptic vesicles have to be retrieved from the membrane by endocytosis, refilled with neurotransmitters, and armed for another exocytosis event. This process is called the synaptic vesicle cycle. It was postulated that all processes in the presynapse directly or indirectly 
provide the means to facilitate the synaptic vesicle cycle and enable fast, regulated, and continuous cycles of neurotransmitter release ${ }^{72}$. As stated in chapter 1.3.1, synapses are defined by the presence of synaptic vesicles in the presynaptic terminal. Apart from a minority of synaptic vesicles that are attached to the active zone, the majority of synaptic vesicles do not observe any morphological characteristics and appear as a homogenous population. Based on release kinetics, it was proposed that there are three distinct pools of vesicles in the synapse: the readily releasable pool, the recycling pool, and the reserve pool. The readily releasable pool constitutes approximately $1 \%$ of synaptic vesicles. It is replenished by the recycling pool, which accounts for 5 to $20 \%$ of all vesicles. The reserve pool constitutes 80 to $90 \%$ of all vesicles. It is only tapped under intense stimulation and rarely recruited during physiological activity ${ }^{73}$.

Synaptic vesicles are filled with neurotransmitters, and dock to the active zone of the presynapse. Priming turns vesicles receptive for a $\mathrm{Ca}^{2+}$ trigger and immediate fusion with the plasma membrane, leading to an exocytosis of neurotransmitters. Recovery of the synaptic vesicle is mediated by three possible ways: the fusion pore opens, closes and the vesicle is replenished with neurotransmitters without undocking ("kiss and stay"); the vesicle undocks and is recycled ("kiss and run"); the vesicle fuses with the membrane completely and synaptic vesicle proteins are retrieved by adaptor proteins and clathrin-mediated endocytosis ${ }^{72,74}$. Figure 1 shows a schematic overview of the synaptic vesicle cycle and involved proteins ${ }^{63}$. In the following chapters, the single steps of the synaptic vesicle cycle and the key proteins involved in spatiotemporal regulation of neurotransmission will be described in detail.

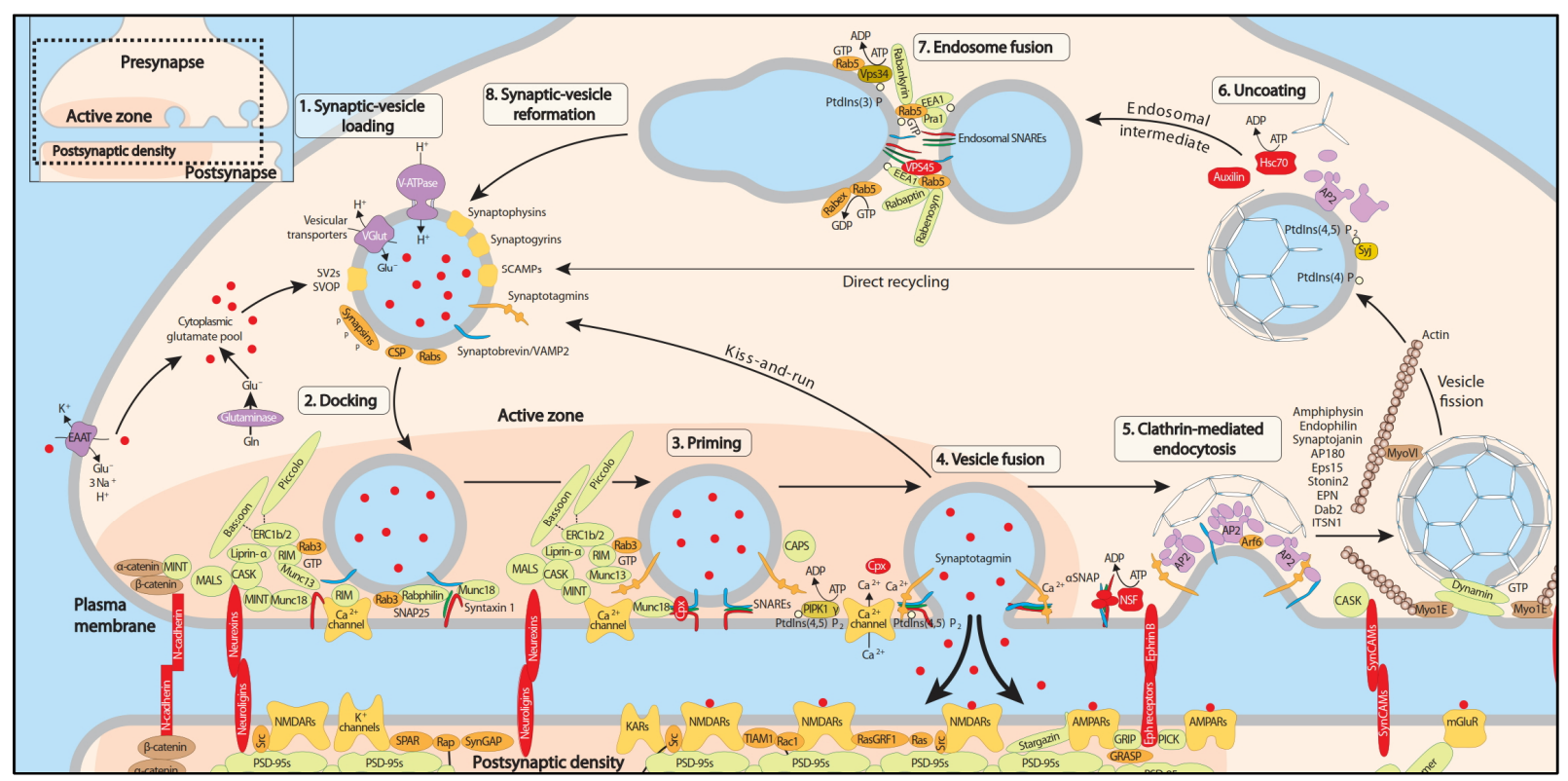

Figure 1: Scheme of the main steps of the synaptic vesicle cycle and involved proteins. See text for details. Image adapted from Chua et al. ${ }^{63}$. 


\subsubsection{Protein composition of synaptic vesicles}

Takamori and colleagues ${ }^{75}$ have developed a molecular model of an average synaptic vesicle. It represents a membrane-enclosed entity of approximately $40 \mathrm{~nm}$ in diameter and its protein composition includes integral and peripheral membrane proteins ${ }^{75}$. The largest multi-subunit protein complex on the surface is the V-type proton transporting ATPase, which pumps protons into synaptic vesicles ${ }^{76}$. The resulting electrochemical gradient across the synaptic vesicle membrane is utilized by vesicular glutamate transporters for neurotransmitter uptake into the vesicle ${ }^{63}$. The most abundant protein is synaptobrevin/VAM P2, a soluble NSF attachment protein receptor (SNARE) protein, whose function is described in chapter 1.3.2.3. Other high abundant constituents are synaptotagmin isoforms, synaptophysin and synapsins. The latter is a phosphoprotein that oligomerizes with itself, tethers synaptic vesicles to each other and maintains synaptic vesicle pools ${ }^{77}$. Synaptophysin is often used as marker protein for synaptic vesicles in electron and fluorescence microscopy ${ }^{78}$. Synaptotagmin 1 is a $\mathrm{Ca}^{2+}$-sensor that, apart from being anchored in the membrane by a transmembrane domain, binds to phospholipids in a $\mathrm{Ca}^{2+}{ }_{-}$dependent manner ${ }^{79}$. It has an important role in $\mathrm{Ca}^{2+}{ }^{2+}$-mediated exocytosis described in chapter 1.3.2.3. Other proteins on the surface of synaptic vesicles are synaptoporins, synaptic vesicle glycoprotein, caM kinase-like vesicle-associated protein (Camkv), and GTP-binding rasrelated in brain (Rab) proteins ${ }^{63,75}$.

\subsubsection{Docking of synaptic vesicles to the active zone}

Synaptic vesicles fuse with the plasma membrane and release neurotransmitters at specialized sites, i.e. active zones. Its constituents are therefore responsible for key functions like synaptic vesicle docking and priming and the recruitment of voltage-gated $\mathrm{Ca}^{2+}$ channels to spatially facilitate signaling. In addition, they are responsible for the exactly opposite localization of the pre- and postsynaptic machinery, which is mediated by cell adhesion molecules ${ }^{80}$. The main components of the active zone scaffold are bassoon, piccolo, Rab3-interacting molecule (RIM), RIM-binding protein (RIM-BP), Munc13, ELKS/RAb6-interacting/CAST family members (ERC), MALS, CASK and $\alpha$-liprin ${ }^{64,80}$. Bassoon and Piccolo are large proteins with redundant functions and no direct involvement in exocytosis ${ }^{81}$. They seem to cluster synaptic vesicles together and are important in guiding vesicles from the reserve pool to release sites under high-frequency stimulation ${ }^{81,82}$. RIM is a central scaffold in the active zone: a ternary complex of RIM, Rab3, and M unc13 recruits synaptic vesicles to the active zone and primes them to a readily releasable state ${ }^{83}$. Acting in concert, RIM and RIM -BP also bind and recruit voltagedependent $\mathrm{Ca}^{2+}$ channels to the active zone ${ }^{80,84}$. At last, another ternary complex consisting of CASK, M int1, and vertebrate homolog of Lin-7 (Veli) connect exocytosis and cell adhesion together by binding to syntaxin-binding protein-1 (Stxbp1/M unc18-1), and cell adhesion molecule neurexin ${ }^{85}$. 


\subsubsection{Synaptic vesicle priming and fusion}

Fusion of synaptic vesicles docked at the active zone is mediated by the assembly of the four-fold alpha-helical SNARE complex. It consists of integral VAM P2 on the vesicle membrane, integral syntaxin1 on the plasma membrane and cytosolic SNAP25, which is palmitoylated and associates with the membrane. It is thought that the zipper-like assembly of the complex towards the membrane anchors pulls the membranes together and provides the energy for membrane fusion ${ }^{86}$. This process is regulated by multiple accessory proteins and is still not understood completely. Docked vesicles are primed, which turns them ready for $\mathrm{Ca}^{2+}$ triggered membrane fusion. The priming process includes the disassembly of already assembled SNARE complexes by N-ethylmaleimide sensitive factor (NSF) and its co-factor $\alpha$-soluble NSF attachment protein ( $\alpha$-SNAP) ${ }^{63,87}$. Stxbp1/M unc18-1 keeps syntaxin-1 in a closed state thereby regulating syntaxin-1 availability for SNARE assembly. M unc13 enables the change of syntaxin- 1 to an open conformation and binding of SNAP $25^{88,89}$. After binding of VAMP2 on the vesicle membrane, the three proteins partially assemble to a trans-SNARE complex, i.e. a pre-fusion state with VAMP2 still on the synaptic vesicle membrane and syntaxin-1 on the plasma membrane. This pre-formed complex is bound and stabilized by multiple synaptotagmin-1 molecules and complexin, which prevents spontaneous full zippering and membrane fusion ${ }^{90}$. With the arrival of an action potential, voltage-gated $\mathrm{Ca}^{2+}$ channels that are clustered at the active zone open and generate a high transient $\mathrm{Ca}^{2+}$ concentration ${ }^{63} . \mathrm{Ca}^{2+}$ is bound by synaptotagmin-1 in the primed pre-fusion complex, which leads to conformational rearrangements and unlocking of the SNARE complex ${ }^{90}$. The latter assembles completely including the membrane anchors of syntaxin-1 and VAMP2, thereby pulling the membranes together and destabilizing the hydrophilic surface of membranes. A fusion pore is formed, which eventually expands and transforms the trans- to a cis-SNARE complex ${ }^{91}$. The neurotransmitter content of the vesicle is released into the synaptic cleft and binds to postsynaptic receptors triggering downstream signaling cascades and an action potential.

\subsubsection{Synaptic vesicle endocytosis and recycling}

Synaptic vesicles that fused with the plasma membrane have to be retrieved and recycled for future neurotransmission events. The cis-SNARE complex is bound by $\alpha$-SNAP and NSF, which disassemble the complex in an ATP-dependent manner ${ }^{87}$. Synaptophysin binds VAM P2 and prevents a reassembly of SNARE complexes ${ }^{92}$. Synaptic vesicle membrane protein clustering and clearance from the neurotransmitter release site is mediated by protein interactions, e.g. oligomerization of synaptophysin and VAM P2, interaction of synaptotagmin- 1 and synaptic vesicle glycoprotein $2 A$, and an unknown effect of vesicular glutamate transporter on other cargo ${ }^{92,93}$. Subsequently, synaptic vesicles are retrieved by clathrin-mediated endocytosis. Adaptor proteins like heterotetrameric adaptor protein 2 (AP-2) complex, and monomeric stonin, epsin, and AP180 bind phosphatidylinositol $(4,5)$ bisphosphate $\left(\mathrm{PI}-4,5-\mathrm{P}_{2}\right)$ on the plasma membrane. In addition, they bind to specific ligand motifs on membrane cargo, e.g. within vesicular glutamate transporter and synaptotagmin-1. Cargo-bound AP-2 stimulates the PIPKI $\gamma$ kinase to generate more PI-4,5-P , which recruits more adaptor proteins ${ }^{94}$. AP-2 also recruits and binds to clathrin leading to clathrin coat assembly and membrane invagination. Clathrin coated vesicles are detached from the membrane by 
the coordinated action of motor proteins like dynamin, myosin $1 \mathrm{E}$ and VI that generate opposing forces along actin filaments ${ }^{63,}{ }^{94}$. Subsequently, synaptic vesicles are uncoated. Synaptojanin 1 binds to endocytosis-related protein endophilin and dephosphorylates $\mathrm{Pl}-4,5-\mathrm{P}_{2}{ }^{95}$. The clathrin cage is disassembled by heat shock cognate $71 \mathrm{kDa}$ protein $(\mathrm{Hsc70})$ and auxilin in an ATP-dependent manner ${ }^{96}$. Other mechanisms for endocytotic retrieval of synaptic vesicles were described, e.g. kissand-run/stay, clathrin-independent endocytosis, and activity-dependent bulk endocytosis, but are less well characterized and/or are subject of controvers ${ }^{93}$. Vesicles finally mature to functional synaptic vesicles via possible intermediate protein sorting steps in endosomes ${ }^{97}$, are loaded with neurotransmitters and can participate in another round of the synaptic vesicle cycle ${ }^{63}$.

\subsubsection{Signal transmission on the postsynaptic side}

Glutamate, which is released from synaptic vesicles, diffuses across the synaptic cleft, and binds to different tetrameric glutamate receptors that are ion channels (ionotropic) and are named after their agonists, e.g. NMDA- (N-methyl-D-aspartate), AM PA- ( $\alpha$-amino-3-hydroxy-5-methylisoxazole-4propionate), and kainate- type receptors. In addition, it can bind to metabotropic glutamate receptors, which are G-protein-coupled receptors. Accordingly, downstream signaling pathways depend on the receptor that binds released glutamate. All ionotropic receptors trigger an excitatory postsynaptic potential, but the speed of channel opening, and cation specificity differs and also depends on the isoform composition of the homo/heterotetramer ${ }^{98}$. Therefore, glutamate binding to the receptors can evoke a postsynaptic action potential by sodium influx, as well as signaling cascades mediated by a transient $\mathrm{Ca}^{2+}$ influx. $\mathrm{Ca}^{2+}$ is quickly cleared from the cytosol to the extracellular side or into intracellular storages by ATP-dependent pumps. Before $\mathrm{Ca}^{2+}$-clearance, ions can bind to proteins that have $\mathrm{Ca}^{2+}$-binding motifs like $\mathrm{C} 2$ domains, e.g. protein kinase $\mathrm{C}$, and E-F-hand motifs, e.g. calmodulin ${ }^{99}$. The latter is usually sequestered by neuromodulin and neurogranin, which regulate the availability of calmodulin under non-stimulated and low $\mathrm{Ca}^{2+}$ conditions ${ }^{100}$. Calmodulin is activated upon $\mathrm{Ca}^{2+}$-binding and undergoes a conformational change that turns it to an activator of various downstream proteins and signaling cascades, for example adenylate cyclase, Ras-guanine nucleotide-releasing factor, neuronal nitric oxide synthase, phosphodiesterase, and calcium/calmodulin-dependent kinase II $(\text { CamkII })^{101}$. The latter oligomerizes to dodecamers and is activated by calcium/calmodulin binding. Subsequent phosphorylation at T286 by neighboring kinase domains keeps the kinase activated independent of a $\mathrm{Ca}^{2+}$ stimulus and is thought to be a form of molecular memory involved in synaptic plasticity, long-term potentiation, and depression ${ }^{102}$.

\subsubsection{Synaptic cytoskeleton and cell adhesion proteins}

The peculiar morphology of neuronal cells with branched dendrites, dendritic spines, axonal ramifications, and synaptic nerve terminals is mediated by a sophisticated system of cytoskeleton proteins and cell adhesion molecules. This system is dynamic, and its reorganization is the molecular basis for neuronal development, and synaptic plasticity ${ }^{103}$. The axon is stabilized by periodic scaffolds of ring-like actin-adducin filaments that are connected by spectrin alpha and beta heterotetramers. 
Voltage dependent sodium channels also follow this periodic localization, which is mediated by ankyrin $G$ binding to sodium channels and spectrin alpha. In contrast, dendrites are characterized by long actin filaments that run along the axis of dendrites ${ }^{104}$. Microtubules similarly traverse along dendrites and axons and serve as infrastructure for motor protein-mediated cargo transport of organelles, vesicles, and proteins that are synthesized in the cell soma and are required at the synapse ${ }^{105}$. Actin and microtubule dynamics in the post-synapse are regulated by NM DA receptor-mediated calcium influx and activation of the Camkll signaling pathway and are thought to drive synaptic plasticity ${ }^{106,107}$. Spectrin alpha also reacts to signaling by directly binding $\mathrm{Ca}^{2+}$ as well as $\mathrm{Ca}^{2+} /$ calmodulin, which was described to promote neuronal plasticity ${ }^{108}$.

\subsubsection{Ion homeostasis in synapses}

Neuronal signal transmission is mediated by changing membrane potentials. For this purpose, ATPdependent ion channels are constantly pumping ions across membranes to restore and maintain ion gradients. The family of P-type ATPases contains members that transport $\mathrm{Ca}^{2+}$ (sarcoendoplasmic reticulum, and plasma membrane $\mathrm{Ca}^{2+}$ transporting $A T P a s e$, SERCA, PMCA), as well as $\mathrm{Na}^{+}$and $\mathrm{K}^{+}$ $\left(\mathrm{Na}^{+} / \mathrm{K}^{+} \mathrm{ATPase}\right)$. This protein family is characterized by an autophosphorylation activity, followed by a consecutive conformation change from an E1 to E2 state, which enables the transport of ions across a membrane ${ }^{109}$. Several model structures of SERCA were reported of the E1 and E2 state as well as substates with and without a phospho-group and binding different ions ${ }^{110-112}$. The most profound conformational changes happen in the cytoplasmic head group of the ATPase, consisting of an actuator $(A)$, nucleotide-binding $(N)$, and phosphorylation domain $(P)$. The head group is wide open in the E1 $2 \mathrm{Ca}^{2+}$ state with the $\mathrm{N}$ - and $\mathrm{A}$-domain far apart from each other. In E2 state, the head group is closed, and the A domain rotated by $110^{\circ}$ into the vicinity of the $\mathrm{N}$-domain. These movements in the head group are triggered by phosphorylation and mediate conformational changes in transmembrane domains. These conformational changes in turn lead to the sequential opening of the ion binding site towards the cytoplasm and the lumen, respectively ${ }^{112}$. PMCA and SERCA are localized in the cytoplasmic and ER membrane, respectively and terminate the transient $\mathrm{Ca}^{2+}$ signal after depolarization by pumping $\mathrm{Ca}^{2+}$ to the extracellular side and the lumen of the ER. The ER and mitochondria are intracellular $\mathrm{Ca}^{2+}$ storage organelles. The latter also plays a role in $\mathrm{Ca}^{2+}$ clearance after prolonged stimulation ${ }^{113}$.

The inositol-1,4,5-trisphosphate receptor (I3PR) is another $\mathrm{Ca}^{2+}$ ion channel, which is of interest for synaptic physiology and which resides mainly in the ER membrane. It is a homotetrameric $1.2 \mathrm{MDa}$ complex that releases $\mathrm{Ca}^{2+}$ from the ER in response to binding of its ligand, which is generated by phospholipase $\mathrm{C}$ as a second messenger in cellular signaling cascades. Subsequent $\mathrm{Ca}^{2+}$ release triggers downstream signaling ${ }^{114}$.

$\mathrm{Na}^{+} / \mathrm{K}^{+}$transporting ATPase, responsible for the $\mathrm{K}^{+}$and $\mathrm{Na}^{+}$balance across the synaptic membrane periodically binds $3 \mathrm{Na}^{+}$ions in E1 state on the cytoplasmic side and releases them on the extracellular side followed by binding of $2 \mathrm{~K}^{+}$ions in E2 state and release on the cytoplasmic side. It generates $\mathrm{Na}^{+}$ and $\mathrm{K}^{+}$ion gradients and a resting potential across the membrane, which is the fundament of the excitability of neuronal cells ${ }^{115}$. In addition, the ion gradient is utilized for transport processes, e.g. the reuptake of glutamate after neurotransmitter release via excitatory amino acid transporters ${ }^{63}$. 


\subsubsection{Synaptosomes as model systems for the study of synapses}

The biochemical research on synapses was pioneered by Victor $P$. Whittaker, who was the first to isolate synaptic nerve endings ${ }^{116}$. Isolation involves brain homogenization, which pinches presynapses off from axons and dendrites. Subsequently, pinched-off synaptic nerve terminals automatically reseal to form so-called "synaptosomes", which can be purified by sequential density gradient and differential centrifugations steps ${ }^{117}$. Electron microscopic studies of synaptosomes morphologically characterized these organelles with the following criteria: They are enclosed by a plasma membrane, contain mitochondria and synaptic vesicles, and frequently exhibit an electron-dense postsynaptic membrane juxtaposed to the presynaptic active zone. This postsynaptic density can occasionally include entire dendrites ${ }^{118}$. In addition, synaptosomes are physiologically active. They take up ions, oxygen, and metabolites like glucose, produce ATP in intra-synaptosomal mitochondria, generate and maintain a membrane potential and neurotransmitter release can be stimulated by membrane depolarization in a $\mathrm{Ca}^{2+}$-dependent manner ${ }^{117}$, 119. The value of synaptosomes for the in vitro study of neurotransmitter release and the action of drugs and toxins was recognized early on ${ }^{118}$. However, synaptosome preparations are inhomogeneous and often contain membranes from myelin fragments, vacuoles of unknown origin, and extra-synaptosomal mitochondria ${ }^{120}$. Different protocols were developed for synaptosome preparation, which differ in the purity and metabolic activity of obtained synaptosomes. For example, preparations involving sucrose density gradient centrifugation constitute a relatively pure synaptosome fraction. However, the osmotic shock of high sucrose concentrations impairs the metabolic activity of synaptosomes, which can be partially restored by incubating synaptosomes in an iso-osmotic solution ${ }^{117,121}$. Utilization of discontinuous Ficoll density gradient centrifugation in isotonic sucrose solutions does not impair synaptosomal activity. Ficoll is a polymer with a high molecular weight that is not osmotically active and does not dehydrate synaptosomes. In addition, shorter centrifugation times are possible with a discontinuous Ficoll gradient, which is beneficial for synaptosome metabolic activity. However, synaptosome fractions obtained with a Ficoll gradient are more contaminated with non-synaptosomal structures in comparison with synaptosomes derived from a sucrose gradient. Only $60 \%$ of the particles in Ficoll gradient-based preparations were recognizable as synaptosomes ${ }^{117,121}$. Neurotransmitter release can be studied in synaptosomes, e.g. by the glutamate release assay ${ }^{119}$. Intra-synaptosomal processes like glutamate uptake into synaptic vesicles is amenable to manipulation by toxin-mediated synaptosome permeabilization ${ }^{122}$. In addition, protocols were established to purify sub-fractions of synaptosomes and to biochemically characterize synaptic proteins ${ }^{123}$. A recent detailed characterization of synaptosomes enabled the generation of a three-dimensional model of a synapse including absolute copy numbers and intra-synaptosomal localization of proteins ${ }^{120}$. 


\subsection{Aim of this thesis}

Synapses are complex structures for intercellular neuronal signal transmission. They contain an intricate and dynamic protein network that enables synaptic vesicle recruitment and docking to the active zone, priming, fusion and retrieval of synaptic vesicle proteins from the plasma membrane. This synaptic vesicle cycle and its underlying dynamic mechanisms are not completely understood so far. The aim of the thesis was to capture and to identify known and novel protein interactions in synaptosomes by cross-linking mass spectrometry. Furthermore, it was intended to quantify changing conformations and protein interactions in response to stimulation by utilizing isotopically labelled cross-linkers, which was not attempted for synaptosomes before.

Cross-linking mass spectrometry analysis of complex samples like synaptosomes is challenging due to the $n^{2}$ problem associated with the identification of cross-linked peptide pairs from fragment ion spectra. The database search space increases exponentially with increasing number of proteins and tryptic peptide candidates in a sample, which compromises the sensitivity of the analysis. In addition, synaptosome preparations are often contaminated with myelin fragments and extra- as well as intrasynaptosomal mitochondria. Accordingly, LC-MS based proteomics analyses of synaptosomes are predominated by mitochondrial and myelin proteins, which is equally challenging for cross-linking mass spectrometry. Therefore, a peptide-focused database search approach was developed that circumvents the $\mathrm{n}^{2}$ problem in cross-linking mass spectrometric analysis of complex samples. Prior to applying the peptide-focused approach on synaptosomes, it was validated on purified cross-linked complexes of known structure and on in vivo cross-linked bacteria. The peptide-focused approach was combined with improved biochemical protocols to deplete myelin and mitochondrial contaminant proteins and enable a stronger focus on cross-linked peptides derived from synaptic proteins. Taking all approaches and workflows together, this thesis aims to present the first qualitative and quantitative cross-linking mass spectrometric analysis of synaptic protein interactions in resting and excited state. 


\section{M aterials and Methods}

\subsection{Materials}

\subsubsection{Chemicals and buffers}

Commonly employed chemicals like salts were obtained from either Sigma-Aldrich (St. Louis, USA), Carl-Roth (Karlsruhe, Germany), Merk M illipore (Billerica, USA), Fluka (Buchs, Germany), or Thermo Fisher Scientific (Waltham, USA). Further chemicals and reagents are listed in Table 1. Commonly used buffers are listed in Table 2.

Table 1: List of used chemicals.

\begin{tabular}{ll}
\hline Chemical & Supplier \\
\hline Acetonitrile, Lichrosolv & Merck Millipore \\
Beta-glycerol phosphate & Sigma-Aldrich \\
Beta-nicotinamide adenine dinucleotide phosphate hydrate (NADP) & Sigma-Aldrich \\
Bond-Breaker TCEP solution (Tris-(2-carboxyethyl)-phosphin) & Thermo Fisher Scientific \\
Chloroform & Merck Millipore \\
Dimethyl sulfoxide (DM SO) & Sigma-Aldrich \\
DSP (dithiobis(succinimidyl propionate)) & Thermo Fisher Scientific \\
DSS-d4, isotopically labelled & Proteochem \\
DSS-No-Weigh Format, (Disuccinimidyl Suberate) & Thermo Fisher Scientific \\
DTT (Dithiothreitol) & Merck Millipore \\
Ficoll 400 & Sigma-Aldrich \\
lodoacetamide (IAA) & Sigma-Aldrich \\
Lauryl maltose neopentyl glycol & Sigma-Aldrich \\
L-glutamic dehydrogenase & Sigma-Aldrich \\
M ethanol, Lichrosolv & Merck Millipore \\
Pepstatin A & Sigma-Aldrich \\
Phosphate Buffered Saline, 10x & Invitrogen \\
Phenylmethylsulfonylfluorid (PM SF) & Roth \\
Trifluoroacetic acid (TFA) & Carl-Roth \\
Trypsin, sequencing grade modified & Promega \\
Water, Lichrosolv & Merck Millipore \\
\hline
\end{tabular}




\subsection{Materials}

Table 2: List of common buffers.

\begin{tabular}{|c|c|}
\hline Buffer & Formulation \\
\hline 20 S proteasome buffer & $\begin{array}{l}100 \mathrm{mM} \text { HEPES, pH 7.5, } 5 \mathrm{mM} \text { beta-glycerol phosphate, } 5 \mathrm{mM} \\
\text { dithiothreitol (DTT), } 5 \% \text { sucrose (w/v) and } 0.01 \% \text { lauryl maltose } \\
\text { neopentyl glycol }(\mathrm{w} / \mathrm{v}) \text {. }\end{array}$ \\
\hline Hypotonic lysis buffer & 5 mM Tris- $\mathrm{HCl}, \mathrm{pH} 8.1$ \\
\hline Lämmli buffer, $1 x$ & $25 \mathrm{mM}$ Tris, $192 \mathrm{mM}$ glycine, 0.1 \% SDS (w/v) \\
\hline Peptide size-exclusion running buffer & $30 \%$ acetonitrile (ACN), $0.1 \%$ trifluoroacetic acid (TFA) (v/v) \\
\hline Phosphate buffered saline (1x, PBS) & $\begin{array}{l}2.67 \mathrm{mM} \mathrm{KCl}, 1.47 \mathrm{mM} \mathrm{KH}_{2} \mathrm{PO}_{4}, 8.06 \mathrm{mM} \mathrm{Na}_{2} \mathrm{HPO}_{4}, \mathrm{pH} 7.4,137.93 \mathrm{mM} \\
\mathrm{NaCl}\end{array}$ \\
\hline RNA Pol II / TFIIH buffer & $\begin{array}{l}150 \mathrm{mM} \text { potassium acetate, } 25 \mathrm{mM} \mathrm{HEPES}, \mathrm{pH} \text { 7.5, } 5 \% \text { glycerol, } 2 \mathrm{mM} \\
\text { magnesium acetate }\end{array}$ \\
\hline Sodium buffer & $\begin{array}{l}140 \mathrm{mM} \mathrm{NaCl}, 5 \mathrm{mM} \mathrm{KCl}, 20 \mathrm{mM} \text { HEPES, pH 7.4, } 5 \mathrm{mM} \mathrm{NaHCO}_{3} \text {, } \\
1.2 \mathrm{mM} \mathrm{Na}_{2} \mathrm{HPO}_{4}, 1 \mathrm{mM} \mathrm{M} \mathrm{gCl}, 10 \mathrm{mM} \text { glucose }\end{array}$ \\
\hline Synaptosome homogenization buffer & $\begin{array}{l}5 \mathrm{mM} \text { HEPES, pH 7.5, } 320 \mathrm{mM} \text { sucrose; } 0.2 \mathrm{mM} \mathrm{PMSF} \text { and } 1 \mu \mathrm{g} / \mathrm{ml} \\
\text { Pepstatin A were added freshly before use }\end{array}$ \\
\hline
\end{tabular}

\subsubsection{Frequently used equipment and consumables}

Table 3: List of consumables used in the study.

\begin{tabular}{ll}
\hline Consumables & Manufacturer \\
\hline C18 microspin columns & Harvard Apparatus \\
Empore C18 extraction disks & 3 M \\
Gel TG PRiM E 4-12\% & Serva \\
NuPAGE 4-12\% Bis-Tris gels & Invitrogen \\
Pierce BCA Protein Assay & Thermo \\
Reprosil-Pur 120 C18-AQ, 1.9 $\mu \mathrm{m}$ & Dr. Maisch \\
SCX cartridge, Strata-X-C & Phenomenex \\
Sep Pak C18 cartridges & Waters \\
\hline
\end{tabular}

Table 4: List of used equipment.

\begin{tabular}{ll}
\hline Equipment & Manufacturer \\
\hline Bioruptor sonication apparatus UCW-201TM & Diagenode \\
Cooling microcentrifuge Heraeus Fresco17 & Thermo Fisher Scientific \\
Freeze dryer alpha 2-4 & Christ \\
Gel chamber Novex M ini-cell & Invitrogen \\
Gradient master mixer B107-202M & Biocomp \\
Incubator BK-600 & Heraeus \\
Incubator M ultitron & Infors-HT \\
Lab scale BP 211D & Sartorius \\
Lab scale CPA 423S & Sartorius
\end{tabular}




\begin{tabular}{ll}
\hline Equipment & Manufacturer \\
\hline LCD Disk-Rotator & Sunlab \\
Mass Spectrometer Q Exactive HF-X & Thermo Fisher Scientific \\
Microcentrifuge Heraeus Pico17 & Thermo Fisher Scientific \\
Nano-LC Dionex UltiM ate 3000 RSLCnano & Thermo Fisher Scientific \\
Optima L-90K Ultracentrifuge & Beckman \\
Peristaltic pump M inipuls 2 & Gilson \\
Power supply power-pac 200 & Bio-Rad \\
Precision scale BP 4100 & Sartorius \\
Rotor SS 34 & Thermo Fisher Scientific \\
Rotor SW 32 & Beckman \\
Rotor SW 41 & Beckman \\
Rotor S100 AT4 & Thermo Fisher Scientific \\
SM ART system & Pharmacia Biotech \\
Sonication bath Sonorex & Bandelin \\
Sorval M TX 150 Micro-ultracentrifuge & Thermo Fisher Scientific \\
Sorvall RC6 PLUS & Thermo Fisher Scientific \\
Spectrophotometer Fluorolog & Horiba \\
Spectrophotometer Nanodrop ND-1000 & Thermo Fisher Scientific \\
Spectrophotometer Ultrospec 3000 & Pharmacia Biotech \\
Speedvac concentrator 5301 & Eppendorf \\
Superdex peptide 3.2/300 size exclusion column & Pharmacia Biotech \\
Thermomixer C & Eppendorf \\
\hline
\end{tabular}

\subsubsection{Software}

Table 5: List of the software used in this study.

\begin{tabular}{ll}
\hline Software & Developer \\
\hline Kojak 1.6.1 & Institute for Systems Biology, Seattle \\
M axquant 1.5.60, and 1.6.0.16 & Cox group, M PI of Biochemistry, M artinsried \\
percolator 3.0 & Käll Lab, KTH Royal Institute of Technology, Stockholm \\
Perseus 1.6.1.2 & Cox group, M PI of Biochemistry, Martinsried \\
pLink 1.23 & pFind Team, Institute of Computing Technology, Beijing \\
pLink 2.35 & pFind Team, Institute of Computing Technology, Beijing \\
Proteome Discoverer 2.1 & Thermo Fisher Scientific \\
PyM OL 1.8.4.1 & Schrodinger, LLC \\
R 3.4 & R core team \\
Rstudio 1.1.463 & Rstudio \\
Tune 2.9 & Thermo Fisher Scientific \\
UCSF Chimera 1.9 & RBVI, University of California \\
Xcalibur 4.1.41.9 & Thermo Fisher Scientific \\
xlinkanalyzer 1.1 & Kosinski Lab, EM BL Hamburg \\
\hline
\end{tabular}




\subsection{Microbiological and biochemical methods}

\subsubsection{Purification and cross-linking of Pol II, TFIIH, and $20 \mathrm{~S}$ proteasome}

Yeast RNA polymerase II (Pol II), and transcription factor IIH (TFIIH) were prepared and generously provided by Dr. Sandra Schilbach (Department of Molecular Biology, Max-Planck-Institute for Biophysical Chemistry, Göttingen, Germany) according to Sydow et al. ${ }^{124}$ and Schilbach et al. ${ }^{125}$, respectively. Human $20 \mathrm{~S}$ proteasome was purified and kindly provided by Dr. Fabian Henneberg (Department of Structural Dynamics, Max-Planck-Institute for Biophysical Chemistry, Göttingen, Germany) as described in Schrader et al. ${ }^{126}$. Complexes were snap-frozen until further use. Pol II complex consisting of 12 subunits Rpb1 to 12 was accommodated in $150 \mathrm{mM}$ K-acetate, $25 \mathrm{mM}$ HEPES, $\mathrm{pH} 7.5,5 \%$ glycerol and $2 \mathrm{mM}$ M g-acetate. TFIIH, a decameric complex, was purified and stored in two parts. One contained Ssl1 and 2, Rad3 and Tfb1 to Tfb5 without Tfb3. The other part contained Kin28, $\mathrm{Ccl} 1$, and Tfb3. Both parts were combined in equal stoichiometries in the same buffer as Pol II and were incubated at $4{ }^{\circ} \mathrm{C}$ for one hour for complex formation prior to cross-linking. $20 \mathrm{~S}$ proteasome consisting of Psa1 to Psa7 and Psb1 to Psb7 was taken up in $100 \mathrm{mM} \mathrm{HEPES,} \mathrm{pH} \mathrm{7.5,} 5$ mM beta-glycerol phosphate, $5 \mathrm{mM}$ dithiothreitol (DTT), $5 \%$ sucrose $(\mathrm{w} / \mathrm{v})$ and $0.01 \%$ lauryl maltose neopentyl glycol $(\mathrm{w} / \mathrm{v})$. All complexes were diluted in their respective buffer without reducing agent to $1 \mu \mathrm{M}$. Small aliquots of complexes were cross-linked with cross-linker amounts ranging from 50x to 2,000x molar excess and were analysed by sodium dodecyl sulphate polyacrylamide gel electrophoresis (SDS-PAGE). An optimal cross-linker concentration was determined at which subunits shifted towards higher molecular weights, but did not disappear completely ${ }^{30}$. Cross-linking of complexes was performed at room temperature with 200 -fold (PoI II), 500 -fold (TFIIH), or 1000 -fold (20 S proteasome) molar excess of disuccinimidyl suberate (DSS), and dithiobis(succinimidylpropionate) (DSP), respectively, for $30 \mathrm{~min}$ or $1 \mathrm{~h}$ in case of $20 \mathrm{~S}$ proteasome. Reactions were quenched with $50 \mathrm{mM}$ Tris- $\mathrm{HCl}$ for $15 \mathrm{~min}$ on ice and Protein complexes were precipitated as described in chapter 2.2.2.

\subsubsection{Protein precipitation}

Protein was precipitated with chloroform/methano| ${ }^{127}$ to remove lipids, DNA and detergents and to exchange the buffer. Briefly, 4 volumes of methanol, 1 volume of chloroform, and 3 volumes of water were added to the sample in succession, each addition followed by vortexing and spinning down the liquid. After adding water, two phases formed, which were separated by centrifugation at $9,000 \mathrm{~g}$ for $1 \mathrm{~min}$. The upper aqueous phase was removed as much as possible without disturbing the protein pellet at the interphase. The phases merged with the addition of another 3 volumes of methanol. After centrifugation for $2 \mathrm{~min}$ at 9,000 $\mathrm{g}$ the liquid was removed and the residual protein pellet was dried under the hood. Subsequent in solution digestion is described in chapter 2.3.1. 


\subsubsection{Bacterial cultivation and in vivo cross-linking}

Single colonies from Bacillus subtilis strain 168 and Bacillus near cereus were picked from a lysogeny broth (LB) agar plate or frozen matter was scratched directly from the glycerol stock and transferred to an overnight culture in LB medium at $37^{\circ} \mathrm{C}$ and $160 \mathrm{rpm}$. Subsequently, a second culture was inoculated 1:50 (v/v) from the overnight culture and grown for approximately $3 \mathrm{~h}$ until reaching an optical density (OD) of 1 at $600 \mathrm{~nm}$ wavelength. $2.4 \mathrm{ml}$ per replicate were harvested, corresponding to estimated $1.2 \times 10^{9}$ cells and $240 \mu \mathrm{g}$ of total protein ${ }^{128,}{ }^{129}$. Cells were washed 1 to 3 times at room temperature with phosphate buffered saline (PBS). The cell suspension was incubated in PBS under rotation at $37^{\circ} \mathrm{C}$ for $15 \mathrm{~min}$ for equilibration, followed by the addition of $1 \mathrm{mM}$ DSS and DSP, respectively. The cross-link reaction proceeded for $10 \mathrm{~min}$ at $37^{\circ} \mathrm{C}$ until it was quenched by adding $50 \mathrm{mM}$ Tris- $\mathrm{HCl}$ and shifting the cells on ice for $15 \mathrm{~min}$. After centrifugation and discard of the supernatant, cells were resuspended in $2 \% \mathrm{SDS}(\mathrm{w} / \mathrm{v})$ and $50 \mathrm{mM}$ Tris- $\mathrm{HCl}$. Cells were disrupted by $30 \mathrm{~s}$ sonication intervals and $15 \mathrm{~s}$ breaks for $20 \mathrm{~min}$ with full power at $4{ }^{\circ} \mathrm{C}$ in a Bioruptor sonication apparatus, followed by $10 \mathrm{~min}$ boiling at $95^{\circ} \mathrm{C}$ and another $10 \mathrm{~min}$ sonication. Small aliquots were taken for SDS-PAGE to check whether the cross-linking reaction was efficient. This was visible as a smear over the entire gel lane. Initially, different durations for cross-linking were tested and evaluated by SDS-PAGE. Protein was precipitated as described in chapter 2.2 .2 and submitted to in solution digestion described in chapter 2.3.1.

\subsubsection{Synaptosome preparation, cross-linking and depletion of myelin fragments and mitochondria}

\subsubsection{Synaptosome preparation}

Synaptosomes were isolated following a previously published protocol ${ }^{119}$ with some modifications. Briefly, 6 to 25 Wistar rats of 5 to 6 weeks age were sacrificed in accordance to the German animal welfare act. Animals were decapitated and cortices and cerebella were dissected from the brains on ice. Samples were kept at $4{ }^{\circ} \mathrm{C}$ throughout the workflow unless stated otherwise. The material was homogenized in $10 \mathrm{ml}$ of homogenization buffer ( $5 \mathrm{mM}$ HEPES, pH 7.5, $320 \mathrm{mM}$ sucrose, freshly supplemented with $0.2 \mathrm{mM} \mathrm{PM} \mathrm{SF} \mathrm{and} 1 \mu \mathrm{g} / \mathrm{ml}$ Pepstatin A, if not stated otherwise) per animal with 9 strokes of a glass-teflon homogenizer at $900 \mathrm{rpm}$. Cell debris and blood clots were removed by 2 min centrifugation at 5,000 rpm in an SS 34 fixed-angle rotor, while the supernatant was re-centrifuged at $11,000 \mathrm{rpm}$ for $12 \mathrm{~min}$. The crude synaptosome pellet was cautiously resuspended with $3 \mathrm{ml}$ of buffer per animal, while avoiding to stir the brown mitochondrial fraction in the pellet in the process. The suspension was loaded on top of discontinuous gradients (one gradient per animal) consisting of $4 \mathrm{ml}$, $1 \mathrm{ml}$ and $4 \mathrm{ml}$ of $13 \%, 9 \%$, and $6 \%(\mathrm{w} / \mathrm{v})$ of Ficoll in homogenization buffer without protease inhibitors, respectively. Gradients were centrifuged for $35 \mathrm{~min}$ at 22,500 rpm in an SW 41 swing-out rotor and the band at the interface of the $13 \%$ and $9 \%$ Ficoll layer was collected. Synaptosome fractions were diluted with homogenization buffer, centrifuged with 11,000 rpm for 12 min in an SS 34 
rotor to get rid of Ficoll and resuspended again with approximately 1 to $1.5 \mathrm{ml}$ homogenization buffer per animal. Either samples were subjected to gradient centrifugation described in chapter 2.2.4.2, or the protein content was determined with a BCA protein assay according to manufacturer's instructions, followed by a glutamate release assay described in chapter 2.2.4.3. Dr. Momchil Ninov (Bioanalytical Mass Spectrometry, Max-Planck-Institute for Biophysical Chemistry, Göttingen, Germany) in part performed synaptosome preparations.

\subsubsection{Depletion of myelin fragments by continuous sucrose gradient centrifugation}

Continuous 25 to $55 \%$ sucrose gradients were prepared as described previously ${ }^{130}$ in SW 32 rotor tubes using standard settings on a Biocomp gradient mixer. Material resulting from two animals was loaded on top of one gradient and was centrifuged for $3 \mathrm{~h}$ at 28,800 rpm in an SW 32 swing-out rotor. Fractions were collected with a peristaltic pump at approximately $0.2 \mathrm{ml} / \mathrm{min}$ flow from the bottom in $2 \mathrm{ml}$ steps and fractions 6 to 12 were pooled and diluted with homogenization buffer. Samples were centrifuged for 12 min at 11,000 rpm in an SS 34 rotor to get rid of high sucrose concentrations and the pellets were resuspended in approximately $0.75 \mathrm{ml}$ homogenization buffer per animal. Subsequently, protein concentration was determined via BCA protein assay according to manufacturer's instructions, followed by evaluation of synaptosome excitability described in chapter 2.2.4.3. Dr. Momchil Ninov (Bioanalytical Mass Spectrometry, Max-Planck-Institute for Biophysical Chemistry, Göttingen, Germany) ran all sucrose gradients.

\subsubsection{Glutamate release assay}

An aliquot of synaptosomes corresponding to $1 \mathrm{mg}$ of protein was centrifuged at $6,000 \mathrm{~g}$ for $3 \mathrm{~min}$ and the supernatant was discarded. The pellet was resuspended in $1 \mathrm{ml}$ of pre-warmed sodium buffer ${ }^{119}$ (140 mM NaCl, $5 \mathrm{mM} \mathrm{KCl}, 20 \mathrm{mM}$ HEPES, pH 7.4, $5 \mathrm{mM} \mathrm{NaHCO}_{3}, 1.2 \mathrm{mM} \mathrm{Na}_{2} \mathrm{HPO}_{4}, 1 \mathrm{mM} \mathrm{M} \mathrm{gCl}$, $10 \mathrm{mM}$ glucose) and was placed in a water bath at $37^{\circ} \mathrm{C}$ for $5 \mathrm{~min}$ for buffer and temperature adaptation. The sample was transferred into a quartz cuvette and supplemented with $1 \mathrm{mM}$ betanicotinamide adenine dinucleotide phosphate hydrate (NADP) and $1.3 \mathrm{mM} \mathrm{CaCl}$, or $0.5 \mathrm{mM}$ EGTA, respectively, for the actual experiment or the control. The cuvette was placed inside the Fluorolog spectrophotometer and the measurement was started. Magnetic stirring was set to a speed of 7 to 8 , and excitation and emission was measured every $2 \mathrm{~s}$ at $330 \mathrm{~nm}$ and $440 \mathrm{~nm}$, respectively, for a total of $1,000 \mathrm{~s}$. Three min into the measurement, $50 \mu \mathrm{l}$ of L-glutamic dehydrogenase was added, followed by $50 \mathrm{mM} \mathrm{KCl}$ after $6 \mathrm{~min}$, which triggered neurotransmitter release. Change in fluorescence emission was measured for roughly 10 min resulting from enzymatic turnover of released glutamate to alphaketoglutarate and generation of NADPH. Data was normalized against the value right before $\mathrm{KCl}$ addition. Dr. Momchil Ninov (Bioanalytical Mass Spectrometry, Max-Planck-Institute for Biophysical Chemistry, Göttingen, Germany) carried out some of the assays. 


\subsubsection{Cross-linking of synaptosomes}

Conditions for synaptosome cross-linking were chosen to closely resemble the glutamate release assay described in chapter 2.2.4.3. Simultaneous cross-linking and assay monitoring via spectrophotometry was not possible due to the reactivity of DSS and DSP towards NADP, which contains primary amines. Synaptosome suspension corresponding to 4 to $5 \mathrm{mg}$ protein content per replicate was centrifuged at $6,000 \mathrm{~g}$ for $10 \mathrm{~min}$. Supernatant was discarded and the synaptosome pellet was resuspended in $1.24 \mathrm{ml}$ of pre-warmed sodium buffer. $1.3 \mathrm{mM} \mathrm{CaCl} 2$ or $0.5 \mathrm{mM}$ EGTA was added for excited or resting conditions, respectively, and synaptosomes were incubated rotating for $10 \mathrm{~min}$ at $37^{\circ} \mathrm{C}$ for buffer and temperature adaptation. Subsequently, the cross-linker was added to a final concentration of $1 \mathrm{mM}$ together with $50 \mathrm{mM}$ of $\mathrm{KCl}$ to trigger glutamate release. DSP or DSS was employed for either peptide database generation, or the actual cross-linking experiment, respectively. For quantification experiments, the two conditions were cross-linked with differentially isotope-labelled DSS-d0, or DSS$\mathrm{d} 4$ containing four deuterium atoms. After $10 \mathrm{~min}$, the reaction was quenched with $50 \mathrm{mM}$ Tris, $\mathrm{pH} 8.1$, for 15 min on ice. Synaptosomes were pelleted for $5 \mathrm{~min}$ at 8,000 $\mathrm{g}$ and the supernatant was discarded. Synaptosomes were either lysed and tryptically digested for LC-MS measurement, or were subjected to synaptosomal membrane enrichment described in chapter 2.2.4.5.

\subsubsection{Depletion of mitochondria by discontinuous sucrose gradient centrifugation}

The workflow described here was modified from Carlin et al. ${ }^{131}$. Synaptosomal pellets were resuspended vigorously in $1.5 \mathrm{ml} 5 \mathrm{mM}$ Tris- $\mathrm{HCl}, \mathrm{pH} 8.1$. For quantification experiments, samples cross-linked at resting conditions were pooled with samples cross-linked at excited conditions with a differentially labelled cross-linker DSS-d0, or DSS-d4, respectively. Conditions and isotopic labels were swapped for different replicates. Synaptosome suspensions were lysed by $15 \mathrm{~s}$ intervals of sonication and cool-down for $10 \mathrm{~min}$ in a Bioruptor sonication apparatus and were placed on top of $9 \mathrm{ml}$ of $5 \mathrm{mM}$ Tris- $\mathrm{HCl}, \mathrm{pH} 8.1$, and $1.05 \mathrm{M}$ sucrose. Samples were centrifuged for $2 \mathrm{~h}$ at 27,600 rpm in an SW 41 rotor. A clear band was visible at the interface of the applied sample and the sucrose cushion, while

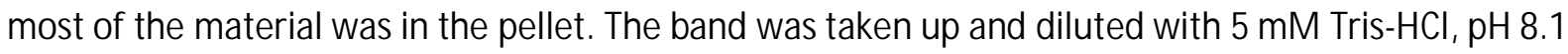
to a final volume of $3.5 \mathrm{ml}$ to get rid of sucrose. Samples were centrifuged for $20 \mathrm{~min}$ at 50,000 rpm in an S100 AT4 rotor and the supernatant was discarded. The pellet containing synaptosomal membranes was resuspended in $1 \% \mathrm{SDS}, 5 \mathrm{mM}$ Tris- $\mathrm{HCl}$, $\mathrm{pH} 8.1$, and heated at $70{ }^{\circ} \mathrm{C}$ for $10 \mathrm{~min}$. A small aliquot was taken for determination of protein concentration by BCA protein assay according to manufacturer's instructions, while the majority was precipitated as described in chapter 2.2.2 and submitted to in solution digestion described in chapter 2.3.1. 


\subsection{Mass Spectrometry and Proteomics methods}

\subsubsection{In solution digestion}

Chloroform/methanol-precipitated protein was dried and resuspended in 40 to $125 \mu \mathrm{l} 8 \mathrm{M}$ urea, depending on the protein amount. Pellets were dissolved by vigorously vortexing and shaking at $1,400 \mathrm{rpm}$, or $10 \mathrm{~min}$ of $15 \mathrm{~s}$ interval sonication in a Bioruptor sonication apparatus, if necessary. $100 \mathrm{mM}$ ammonium bicarbonate (ABC, $50 \mathrm{mM}$ final concentration) was added and the sample was again shaken, or sonicated, if necessary. Following, the same volume of buffers, i.e. between 80 and $250 \mu \mathrm{l}$ depending on the starting volume, were added per pipetting step to dilute urea to $1 \mathrm{M}$ final concentration prior to trypsin addition. To reduce cysteines and disulphide bridges within the DSP cross-linker, protein suspensions were mixed with $10 \mathrm{mM}$ dithiothreitol (DTT) in $50 \mathrm{mM} \mathrm{ABC}$ to a final concentration of $5 \mathrm{mM}$ and incubated for $30 \mathrm{~min}$ at room temperature and 1,400 rpm. Sulfhydryl groups were alkylated with $55 \mathrm{mM}$ iodoacetamide (IAA, $18.33 \mathrm{mM}$ final concentration) in $50 \mathrm{mM} \mathrm{ABC}$ for 30 min at 1,400 rpm in the dark. Finally, residual IAA was quenched by adding $10 \mathrm{mM}$ DTT $(2.5 \mathrm{mM}$ final concentration) in $50 \mathrm{mM} \mathrm{ABC}$. DTT and IAA was omitted for DSP cross-linked synaptosomes and bacteria for keeping cross-links intact and being able to enrich them as described in chapter 2.3.3. Trypsin digestion was performed overnight rotating at $37^{\circ} \mathrm{C}$, or room temperature to reduce carbamylation side reactions. Purified complexes were digested with a 1:20 (w/w) trypsin to protein ratio, while a 1:50 (w/w) ratio was utilized for bacteria and synaptosomes. The digest was stopped by adding $0.5 \%$ trifluoroacetic acid (TFA) and $5 \%$ acetonitrile (ACN, v/ v), followed by desalting described in chapter 2.3.2.

\subsubsection{Desalting}

C18 cartridges or microspin columns were wetted and equilibrated with 500 or $200 \mu$ l of solvents, respectively, in the following order: methanol, $\mathrm{ACN}, 50 \% \mathrm{ACN}$ and $0.1 \% \mathrm{TFA}(\mathrm{v} / \mathrm{v})$, and three times $5 \%$ ACN and $0.1 \%$ TFA (v/v). Samples were applied and passed by gravity. Bound peptides were desalted three times with $5 \% \mathrm{ACN}$ and $0.1 \%$ TFA (v/v) and eluted twice with $80 \% \mathrm{ACN}$. Eluates were snap-frozen and kept at $-80{ }^{\circ} \mathrm{C}$ until further use. 


\subsubsection{Cross-linked peptide enrichment}

\subsubsection{Enrichment by strong cation exchange chromatography}

Cross-linked peptide enrichment by polymeric strong cation exchange (SCX) chromatography was based on a previously published protocol ${ }^{132}$. Desalted peptides were lyophilized almost completely and resuspended by vigorous shaking in $10 \%$ methanol, $0.5 \%$ acetic acid. Meanwhile, the polymeric SCX Strata-X-C cartridge was wetted and equilibrated with $1 \mathrm{ml}$ of solvents in the following order: methanol, $0.5 \%(\mathrm{v} / \mathrm{v})$ acetic acid, $2.5 \mathrm{M}$ ammonium acetate in $80 \%(\mathrm{v} / \mathrm{v})$ methanol, and two times $0.5 \%(\mathrm{v} / \mathrm{v})$ acetic acid. The sample was applied twice by gravity and non-bound peptides were removed with $2 \mathrm{ml} 0.5 \%(\mathrm{v} / \mathrm{v})$ acetic acid. Subsequently, peptides were eluted in two fractions with $1 \mathrm{ml} 1.5 \mathrm{M}$ ammonium acetate in $50 \%$ methanol $(\mathrm{v} / \mathrm{v})$ and $2.5 \mathrm{M}$ ammonium acetate in $80 \%$ methanol $(\mathrm{v} / \mathrm{v})$, respectively. Fractions were snap-frozen and dried for $3 \mathrm{~h}$ down to 100 to $150 \mu \mathrm{l}$, at which it was assumed that all methanol evaporated and only water and ammonium acetate was left, which substantially slowed down the evaporation process. Samples were acidified to $\mathrm{pH} 2$ with TFA, filled up to $1 \mathrm{ml}$ with water and desalted as described in chapter 2.3.2.

\subsubsection{Enrichment by peptide size-exclusion chromatography}

Cross-linked peptides were enriched as described before ${ }^{133}$. Briefly, Peptides were lyophilized, resuspended in running buffer consisting of $30 \% A C N, 0.1 \%$ TFA (v/v), and injected on a Superdex peptide 3.2/300 size-exclusion column, operated by a SM ART chromatography system. Peptides were separated with a $50 \mu \mathrm{l} / \mathrm{min}$ flow. Fractions of $25 \mu \mathrm{l}$ between 1.075 and $1.35 \mathrm{ml}$ elution volume were collected. The first three fractions within this window were pooled as well as the three last fractions, respectively, resulting in a total of seven fractions, which were finally dried.

\subsubsection{Cleavage of disulphide bridges in DSP-cross-linked samples}

Peptide size-exclusion fractions of DSP cross-linked bacteria and synaptosomes were resuspended in $100 \mathrm{mM} \mathrm{ABC}, 10 \mathrm{mM}$ Tris-(2-carboxyethyl)-phosphin (TCEP), and $3 \% \mathrm{ACN}(\mathrm{v} / \mathrm{v})$, sonicated and incubated at $56{ }^{\circ} \mathrm{C}$ for $30 \mathrm{~min}$. Samples were shortly put at room temperature to cool down, after which $20 \mathrm{mM}$ IAA was added to alkylate newly-formed thiol groups from the cleaved cross-linker for $30 \mathrm{~min}$ at room temperature in the dark. Alkylation was stopped by the addition of $0.5 \%$ TFA (v/v). Samples were finally desalted with custom-built C18 StageTips ${ }^{134}$. Briefly, three to four small disks of $1 \mathrm{~mm}$ diameter were punched out of teflon C18 solid phase extraction material and were stacked within a $200 \mu \mathrm{l}$ pipette tip. Desalting was performed similar to chapter 2.3.2 with the exception of passing $50 \mu \mathrm{l}$ volumes of solvents through the resin by centrifugation. Eluates were snap-frozen and stored at $-80^{\circ} \mathrm{C}$ until LC-M S measurement. 


\subsubsection{Mass spectrometric acquisition}

All measurements were performed on a Q Exactive HF-X hybrid quadrupole-Orbitrap mass spectrometer with a Dionex UItiM ate 3000 RSLCnano system at the front. Chromatographic separation was achieved on a C18 PepM ap100 $\mu$-Precolumn $(0.3 \times 5 \mathrm{~mm}, 5 \mu \mathrm{m})$, and an in-house packed main column $(75 \mu \mathrm{m} \times 30 \mathrm{~cm})$ at a flow of $300 \mathrm{nl} / \mathrm{min}$.

Peptides from purified protein complexes cross-linked with DSS were dried and resuspended in $5 \%$ ACN , $0.1 \%$ TFA (v/v) and analyzed in technical duplicates, or triplicates. Gradients were performed for 165 min from $10 \%$ to $50 \%$ buffer B ( $80 \%$ ACN, $0.08 \%$ formic acid, v/v) and an overall 180 min method duration. Precursor ion scans were acquired with 120,000 resolution, $1 \times 10^{6}$ automatic gain control (AGC) target, and $50 \mathrm{~ms}$ maximum injection time from 380 to $1,580 \mathrm{~m} / \mathrm{z}$. The 20 most abundant precursor ions were selected with a $1.4 \mathrm{~m} / \mathrm{z}$ isolation window and were fragmented with a normalized collision energy of 30 . Fragment ion scans were acquired with 30,000 resolution, $5 \times 10^{3}$ minimum and $2 \times 10^{5}$ target AGC, $200 \mathrm{~ms}$ maximum injection time and a fixed first mass of $110 \mathrm{~m} / \mathrm{z}$. Only precursor charges between 3 and 7 were included and precursors, that were fragmented once, were excluded from reselection for $5 \mathrm{~s}$.

Purified protein complexes cross-linked with cleaved and alkylated DSP were analyzed similarly with the following changes: Peptides were resuspended in 3\% ACN, $0.1 \%$ TFA (v/v). The gradient was ranging from $3 \%$ to $36 \% \mathrm{~B}$ during the first $155 \mathrm{~min}$, followed by a steeper gradient to $45 \% \mathrm{~B}$ for $13 \mathrm{~min}$. M S1 scans were ranging from 300 to $1,600 \mathrm{~m} / \mathrm{z}$. Fragment ion scans of the 30 most abundant precursors with charges 2 to 6 were acquired with 15,000 resolution and maximum 128 ms injection time. Once fragmented precursors were excluded from selection for $10 \mathrm{~s}$.

Peptide size-exclusion fractions of DSS cross-linked bacteria and synaptosomes were separated by 75 min gradients (90 min total run time) of varying percentage of buffer B to account for a proportionality between size and hydrophobicity. Gradients were ranging from $20 \%$ to $50 \%$ and $16 \%$ to $48 \%$ B for early fractions and $14 \%$ to $46 \%$ and $12 \%$ to $45 \%$ B for late eluting fractions. MS acquisition settings were identical to DSS cross-linked purified protein complexes, except for $128 \mathrm{~ms}$ maximum injection time and $10 \mathrm{~s}$ dynamic exclusion.

Cleaved and alkylated fractions of DSP cross-linked bacteria and synaptosomes were separated by a 71 min gradient from $3 \%$ to $36 \%$ B, followed by a gradient to $45 \%$ B for 7 min. M S acquisition settings were identical to DSP cross-linked purified protein complexes with following exceptions: $60,000 \mathrm{MS1}$ resolution, 54 ms maximum injection time, a loop count of 40 and $30 \mathrm{~s}$ dynamic exclusion. 


\subsection{Data processing and analysis}

\subsubsection{Proteomics label-free quantification analysis of myelin- and mitochondria-depleted synaptosomes}

Analysis of linear (non-cross-linked) peptides of synaptosomes after myelin depletion (chapter 2.2.4.2) and mitochondria depletion (chapter 2.2.4.5) was performed using M axquant ${ }^{135}$ with default settings. Each bioreplicate of a fraction after depletion and its corresponding control without a depletion step was defined as a separate experiment. Label-free quantification and the match-between-runs option was enabled, and raw files were searched against the Uniprot rattus norvegicus reference proteome $(29,806$ entries, retrieved on $06 / 07 / 17)$.

\subsubsection{Peptide database generation}

Raw acquisition files of purified cross-linked complexes were submitted to a Maxquant database search against the fasta sequences of the proteins involved in the respective complexes. Cross-linked $20 \mathrm{~S}$ proteasome samples were first searched against the entire human proteome. A fasta database was built from ActB, Cap1 and p97 in addition to the fourteen subunits of the proteasome, reflecting the most abundant and most likely proteins to be involved in cross-links.

Cross-linked bacteria and synaptosomes were searched against the respective Uniprot reference proteomes (Bacillus subtilis strain 168, 4264 entries, retrieved on 07/23/18; Bacillus cereus strain ATCC 14579, 5240 entries, retrieved on 07/24/18; rattus norvegicus as stated in chapter 2.4.1). Database search was performed with default settings with the following exceptions below. The cleaved and alkylated DSP cross-linker modification was defined as variable (145.0197 Da; H(7) C(5) N O(2) S) on lysines. Minimum peptide length was lowered to 5 amino acids and maximum peptide mass was increased to $10,000 \mathrm{Da}$. Three trypsin missed cleavages were considered. In the interest of obtaining a near complete coverage of peptides involved in cross-links, protein, PSM and site false discovery rate (FDR) was put to 0.1 and minimum score and delta score for modified peptides was set to 0 . The accompanying increase of false-positives was considered negligible, since it only increased the size of the resulting peptide database and not necessarily compromised the FDR calculation of the cross-link analysis.

The resulting peptides.txt table was used to generate a database consisting of peptides that were identified with a cleaved DSP cross-link modification using a custom R script. Peptide entries from reverse and contaminant proteins were filtered and only peptides with an entry in the cleaved DSP site ID column were retained in the list, i.e. peptides that were identified with a cleaved DSP cross-link modification. For cross-linked bacteria and synaptosomes, peptides with 0 and 3 missed cleavages were filtered out, whereas for purified complexes, peptides with 3 missed cleavages were retained. The remaining peptides were concatenated with a unique identifier, which consisted of a uniprot accession number of the corresponding protein and an integer, and a newline in between, thereby following the FASTA nomenclature. 
Direct use of the resulting database with any cross-linking search algorithm that automatically generates decoys by sequence reversal would lead to a biased target-decoy competition. Since sequence reversal would be performed post in silico digestion, all decoy sequences would have an $\mathrm{N}$ terminal lysine or arginine and would inherently differ from target sequences. In addition, all decoy sequences would have the same molecular composition, whereas approximately half of all peptide decoy sequences from reverted proteins would have a different molecular mass due to an altered tryptic end. Therefore, decoy peptide sequences were generated resembling sequences that were first reversed and subsequently in silico digested. C-terminal lysine or arginine from target peptides was omitted, the sequence was reversed and the respective amino acid from the 'amino acid before' column was appended. In case of protein C-terminal peptides lacking a tryptic end, no amino acid was omitted. Moreover, no amino acid was appended in case of protein N-terminal peptides. Decoy sequences that were identical to target sequences (e.g. a palindromic sequence), were shuffled. The resulting decoy sequences were combined with a unique header containing a "REVERSE_"-tag, appended to the target sequences and saved as fasta file.

\subsubsection{Cross-link database search with pLink, and Kojak}

Raw acquisition files of DSS cross-linked samples were converted to mgf format with Proteome Discoverer and were subjected to a database search with pLink $1^{41}$, or pLink 2 against the sequences of the respective protein complexes, proteomes, or generated peptide databases.

The conventional database search of the $20 \mathrm{~S}$ proteasome sample against its protein sequences was performed with reversed sequences of Pol II subunits and glutamate dehydrogenase as decoys with a similar number of decoy peptide candidates. Low identification numbers were observed with decoys derived from $20 \mathrm{~S}$ proteasome protein sequences.

The following parameters were applied: Cross-linker DSS (138.068 Da), and DSS-d4 (142.093 Da) for quantitative experiments, Carbamidomethylation on cysteine as fixed and oxidation on methionine as variable modification, peptide lengths between 6 and 100 amino acids, peptide masses between 400 and $10,000 \mathrm{Da}, 10 \mathrm{ppm}$ precursor mass tolerance, three missed cleavages for purified protein complexes, and 2 missed cleavages for cross-linked bacteria and synaptosomes, open-search mode (search_mode=1), and 1\% FDR cut-off on spectral level. For peptide database searches, enzyme specificity was set to $\mathrm{C}$-terminal cleavage after $\mathrm{B}$, i.e. peptides were not in silico digested and taken as is. DSS cross-link specificity was limited to lysine to lysine links omitting protein $\mathrm{N}$-terminal reactivity, or else peptide N-termini would be interpreted as additional linkage sites.

Resulting spectral identifications referred to artificial protein headers with wrong linkage sites, which were transformed to correct protein accession numbers and linkage sites with a custom R script. The unique identifier was used to retrieve the correct protein header from the proteinGroups.txt table and the start and end positions of the two peptides in the peptides.txt table to determine the correct linkage sites in the protein.

In addition, raw acquisition files of DSS cross-linked bacteria and synaptosomes were analyzed with Kojak ${ }^{42}$ version 1.6.1. Cross-link specificity, missed cleavages, precursor mass tolerance, and modifications were set identical to pLink along with the following settings: Isotope error of 2, peptide masses between 500 and 10,000 Da, use of 200 peaks per spectrum, supplemented precursor prediction, and a top_count of 30. FDR analysis of the Kojak output was performed on inter and intra 
links jointly with percolator ${ }^{136}$ after excluding peptides shorter than 6 amino acids. Identifications were reported with a $1 \%$ FDR cut-off.

\subsubsection{Quantification of cross-links}

Result tables from pLink database searches were reformatted according to the needs of XiQ quantification software described in Fischer et al. ${ }^{1}$. Quantification was performed by extracting areas under the curve from raw files of the first to third isotopic peak delimited by a drop to $10 \%$ signal intensity and dividing the average of heavy peaks by the average of light peaks. Quantitative values were $\log _{2}$ transformed, median normalized, and the leading sign of ratios was changed, in case of labelswap experiments, to have a ratio of excited to resting synaptosomes in all bioreplicates. Subsequently, quantified redundant spectral assignments were condensed to unique linked sites with an R script based on a previous publication ${ }^{56}$. First, median ratios were calculated for each charge state per peptide, which were then summarized to unique peptides as a weighted average. Unique peptides were finally summarized to unique linked residues as median ratios of all supporting peptides.

\subsubsection{Data analysis and visualization}

Label-free quantification of myelin- and mitochondria-depleted synaptosomes with three bioreplicates each was processed with Perseus software. Contaminants, reverse hits, and identifications based on only modified peptides were filtered out. Data was $\log _{2}$ transformed and a two-sided t-test was applied with $5 \%$ FDR and an artificial within groups variance of 1 . Not all proteins of the respective group needed to be labelled for the observation of mitochondrial and synaptic proteins. Exemplarily proteins with the term 'mitochondrial' in their fasta header were labelled as such in the respective plot. Likewise, synaptic proteins (in total 118) were chosen as representatives from proteins identified in a previous study ${ }^{130}$ with the following gene ontology terms: Active zone proteins, adhesion and cell surface molecules, endocytosis-related proteins, postsynaptic, SNARE and trafficking proteins, synaptic vesicle proteins, channels, transporter and receptors.

Identified cross-links of protein complexes, bacteria and synaptosomes, were visualized with xiNET ${ }^{137}$, and were plotted on available crystal structures with xlinkanalyzer ${ }^{46}$ within Chimera, or with pyM OL, respectively. A pLink score cut-off and a minimum number of spectral identifications was applied for purified protein complexes in addition to the FDR cut-off, as stated in the figure legends. Inter-links of cross-linked bacteria and synaptosomes were manually checked for plausibility, e.g. whether an interaction is reported in the literature, if proteins reside in the same compartment, or if they have a related function, while also considering the pLink score and the number of spectral hits.

Quantitative cross-link data consisted of three bioreplicates and three cross-link reaction replicates, each. Ratios were filtered for at least three valid values per cross-linked residue pair and a two-sided t-test was performed with 0 as $\mathrm{H}_{0}$-hypothesis and a $\mathrm{p}$-value threshold of 0.05 . Fold-change difference below 1.5 were considered to be insignificant. 


\section{Results}

\subsection{Peptide-focused cross-linking database search approach}

The cross-linking analysis of a complex sample like synaptosomes cross-linked with a non-cleavable cross-linker, e.g. BS3 and DSS, is challenging. The database search space exponentially increases with the number of peptide sequences derived from a database ${ }^{28}$. A large search space derived from mammalian proteomes is accompanied by many false positive identifications and a low sensitivity ${ }^{47}$. Therefore, a workflow was developed that circumvents the so-called $n^{2}$ problem that is observed in XL-MS (described in 1.2.2). Peptides, which were involved in cross-links, were identified in advance, and were provided for database search, instead of considering all theoretically cross-linkable peptides. The sample was split, and two cross-linking reactions were performed in parallel with a non-cleavable and thiol-cleavable cross-linker with equal reactivity and similar spacer length (figure 2)

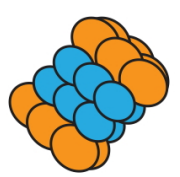

Purified protein complexes
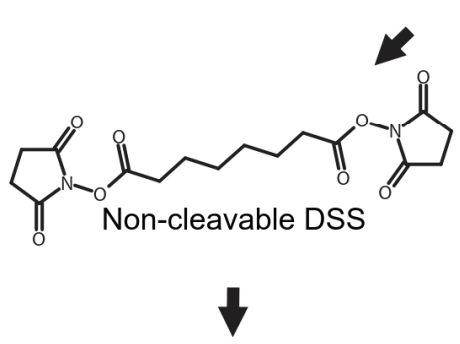

Reduction \& alkylation

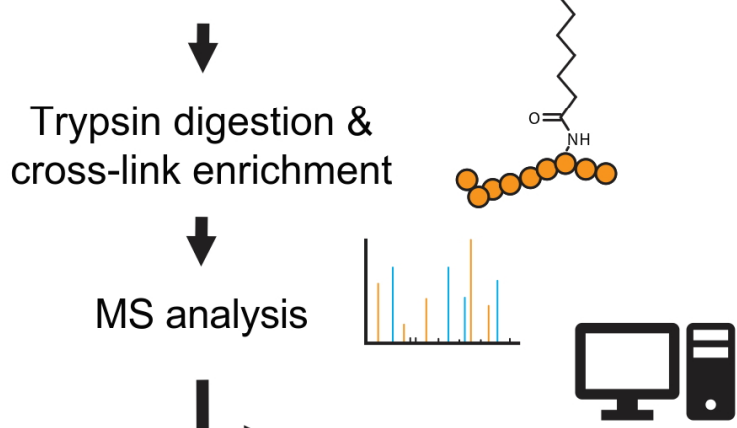

Cross-link database search

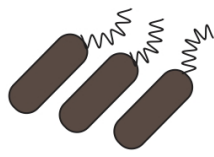

Bacteria
Sample preparation

Cross-linking

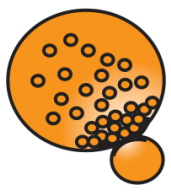

Synaptosomes<smiles>O=C(CCSCCC(=O)ON1C(=O)CCC1=O)ON1C(=O)CCC1=O</smiles>

Trypsin digestion \& cross-link enrichment

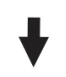

Reduction \& alkylation
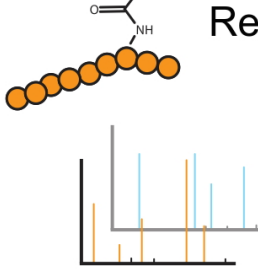

MS analysis

Peptide database generation

Figure 2: Schematic representation of the peptide-focused cross-linking workflow. See text for details. 
The sample cross-linked with the non-cleavable linker was reduced and alkylated, and proteins were tryptically digested. After enrichment of cross-linked peptides, the latter were analyzed by LC-MS (figure 2, left side). The thiol-cleavable counterpart was tryptically digested without prior reduction and alkylation. Thiol-cleavable DSP cross-links were therefore kept intact. Only after tryptic digestion and cross-link enrichment, the cross-linked peptides were cleaved by reduction and alkylation and analyzed by LC-MS (figure 2, right side). An initial Maxquant $^{9}$ database search against the Rattus norvegicus database was performed and the cleaved and alkylated DSP cross-linker was set as variable modification on the reactive lysine. This search served the purpose of identifying the proteins, to which the modified peptides belonged. The latter ones were extracted and merged in a much smaller database that was used for the analysis of the actual sample treated with a non-cleavable cross-linker. Due to a smaller search space and a focus on peptides that were experimentally shown to be involved in cross-links, it was hypothesized that the analysis time and false positive rate is decreased, thereby enabling the cross-link analysis of complex mammalian proteomes.

\subsection{Application of the peptide-focused approach on purified complexes}

Prior to applying the approach described in chapter 3.1 on complex samples like synaptosomes, its integrity was tested on less complex samples with a reported structural model. identified cross-linked residues can be mapped on the respective structure to measure the $\mathrm{C} \alpha-\mathrm{C} \alpha$ atom distance. As described in chapter 1.2.2, DSS cross-linkers can span a $\mathrm{C} \alpha$-C $\alpha$ atom distance of up to $30 \AA$. Distances longer than $30 \AA$ are either false positive identifications, or an indication for conformational flexibility. A C $\alpha-C \alpha$ atom distance below $30 \AA$ on a model structure can provide an additional assessment whether a reported cross-link is a true positive identification.

Therefore, human $20 \mathrm{~S}$ proteasome ${ }^{126}$ with co-purified transitional endoplasmic reticulum ATPase ${ }^{138}$ (p97), yeast polymerase $\mathrm{II}^{125}$ (Pol II), and transcription factor IIH ${ }^{125}$ (TFIIH) were processed individually as explained in chapter 3.1. Pol II and TFIIH were prepared and generously provided by Dr. Sandra Schilbach (Department of Molecular Biology, Max-Planck-Institute for Biophysical Chemistry, Göttingen, Germany). 20 S proteasome and p97 were purified and kindly provided by Dr. Fabian Henneberg (Department of Structural Dynamics, Max-Planck-Institute for Biophysical Chemistry, Göttingen, Germany).

Complexes were cross-linked with non-cleavable DSS and thiol-cleavable DSP. The latter was cleaved and alkylated during the workflow. Samples were analyzed by LC-MS and a peptide database was generated for each complex based on identified peptides with a cleaved cross-linker. Subsequently, LC-M S data derived from complexes cross-linked with non-cleavable DSS was searched against the respective protein sequences, termed the "conventional" approach. In parallel, the same LC-M S data

was also searched against the peptide database, termed the "peptide-focused" approach. Spectral identifications, termed "cross-link to spectrum match" (CSM) are summarized in table 6. 
Table 6: Number of CSM s identified with the conventional and peptide-focused approach for purified complexes.

\begin{tabular}{rcc}
\hline Identified CSM s per complex and approach & Conventional approach & Peptide-focused approach \\
\hline Human 20 S proteasome and p97 & 6,193 & 5,279 \\
Yeast Pol II & 4,553 & 3,689 \\
Yeast TFIIH & 4,468 & 4,032 \\
\hline
\end{tabular}

For example, 4,553 CSM s were identified for yeast Pol II with the conventional approach. The peptidefocused approach yielded 3,689 CSM s. In terms of cross-link identification numbers, the peptidefocused strategy did not outperform the conventional database search for these complexes.

The $\mathrm{C} \alpha-\mathrm{C} \alpha$ atom distances between identified cross-linked lysine residues were measured on the respective crystal structures and the distribution was plotted in figure 3 and supplemental figure 1. Multiple identified CSM s describing the same cross-linked lysine residue pair were collapsed to unique cross-linked residues. A pLink score and CSM cut-off was applied individually for each complex to exclude identifications with low confidence and to compare high confidence identifications only. Higher pLink scores mean more confidence in the identification. Comparing both approaches, the number of identified unique cross-linked residues and the distribution of $\mathrm{C} \alpha-\mathrm{C} \alpha$ atom distances was very similar between both approaches (figure 3 and supplemental figure 1 ). The peptide-focused approach did not result in additional overlength cross-links greater than $30 \AA$ with the exception of one cross-link for $20 \mathrm{~S}$ proteasome (figure $3, \mathrm{~A}$ ). Five more unique cross-linked residues with a minimum pLink score of five were identified with the peptide-focused approach, though. No additional or even one less overlength cross-link was identified for $\mathrm{p} 97$ and TFIIH, respectively (supplemental figure 1). The number of overlength cross-links was rather depending on the sample and complex than on the analysis strategy. $20 \mathrm{~S}$ proteasome, which constitutes a stable barrel-shaped complex, yielded almost no cross-links greater than $30 \AA$, whereas Pol II and TFIIH have a less compact structure and returned 6 to $9 \%$ overlength cross-links. In contrast to previous structural studies ${ }^{125}$ of Pol II and TFIIH, no protein size exclusion chromatography step was performed after complex assembly and crosslinking here. Such a step would eliminate denatured and precipitated complexes, which are a source for overlength cross-links.

It was reasoned that the difference in identified CSM s between the two approaches (table 6) was partially a consequence of an incomplete peptide database. Peptides involved in cross-links that were exclusively identified by the conventional approach tended to be very short or very long. For example, peptides of four and five amino acids length as well as peptides longer than 50 amino acids were often absent from the peptide database and were therefore not identified. Very short and very long peptides are not covered by default database search settings, which led to the modifications described in chapter 2.4.2. Furthermore, both the cross-linking reaction and the LC-M S analysis in data-dependent acquisition mode are inherently stochastic processes, meaning that two independently prepared crosslinking reactions will not bear the same set of identified cross-links and every LC-MS acquisition will lead to slightly different identifications. Indeed, acquiring an additional DSP-cross-linked and cleaved replicate of Pol II expanded the peptide database and led to $43 \%$ more identified CSM s. Thus, it is advisable to perform multiple experimental replicates to expand the peptide database. A majority of $70 \%$ of the cross-linked peptides, which were identified by the conventional approach but not by the peptide-focused approach, were indeed not present in the peptide database. Possible reasons for an incomplete database are discussed in detail in chapter 4.1.2. The remaining $30 \%$ had a low average pLink score of 2.6 in comparison to an average 6.7 score for cross-links identified by the conventional approach. A low average pLink score indicated the presence of low-quality CSMs in that subset. 
However, as short peptides have a poor fragmentation pattern and often cannot be assigned unambiguously to one protein, the minimum peptide length was raised to six amino acids for crosslinking database search.

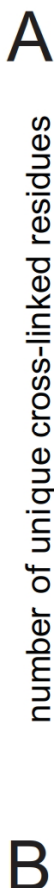
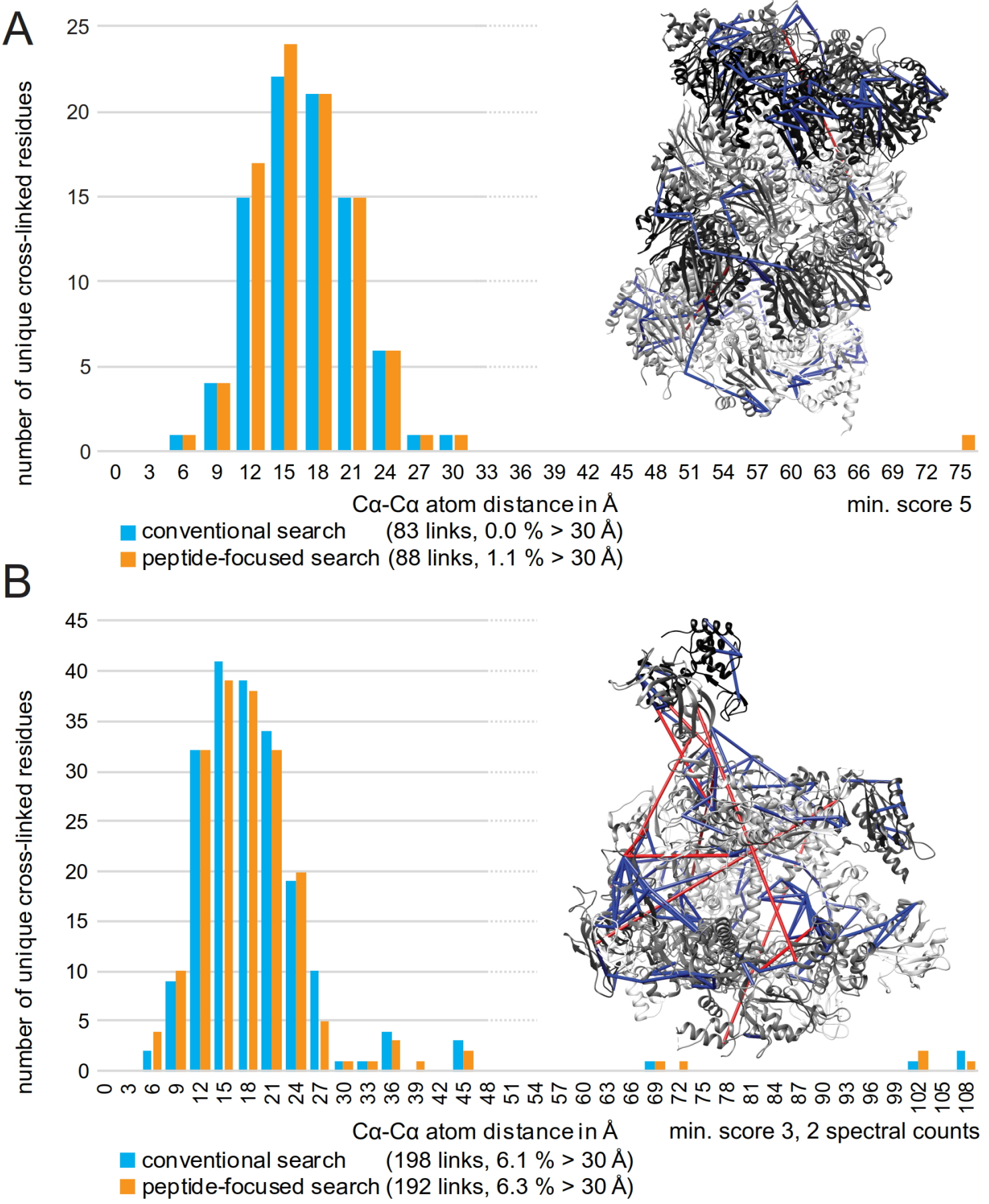

Figure 3: Comparison of conventional cross-linking with the peptide-focused approach on purified proteins. 20 Sproteasome $(A, p d b: 5 L E 5)^{126}$, and Pol II (B, pdb: 50QM $)^{125}$ were processed as outlined in chapter 3.1 and resulting cross-links were filtered for a minimum pLink score of 5 ( 20 S proteasome) or 3 and at least 2 identified CSM s (Pol II). C $\alpha$-C $\alpha$ atom distances between cross-linked lysines are plotted as histogram and are exemplary shown on the respective crystal structure (grey to black) for the peptide-focused approach. Cross-link distances greater than $30 \AA$ are considered overlength and are depicted in red. 
It is possible to cross-link the sample with thiol-cleavable DSP and perform the cross-linking analysis and peptide database generation with the same sample. Splitting the sample early during the workflow and cross-linking with different agents might have the disadvantage that any variation occurring thereafter, including cross-linker reactivity, will accumulate and ultimately lead to a peptide database, which poorly represents the actual cross-linking sample. To overcome this vulnerability, it appeared reasonable to cross-link the sample only with thiol-cleavable DSP and split the sample after cross-link enrichment. One part was analyzed directly LC-M S, while the other was reduced and alkylated followed by LC-MS analysis for peptide database generation. This approach seemed unfeasible, because DSP cross-links scattered strongly (figure 4). Comparing cross-links obtained with DSS and DSP for Pol II, the latter scattered over the entire structure of Pol II. The majority of identified cross-linked residues had a $\mathrm{C} \alpha-\mathrm{C} \alpha$ atom distance larger than $30 \AA$. The scattering was probably due to a reducing agent being present initially in the sample and due to an alkaline $\mathrm{pH}$ during digestion ${ }^{139}$. Thus, cross-links could interchange randomly. As a consequence, the alternative approach with only DSP was abandoned, since the eventual goal was to apply the peptide-focused approach on complex samples like synaptosomes, which feature a reducing environment in the cytoplasm.

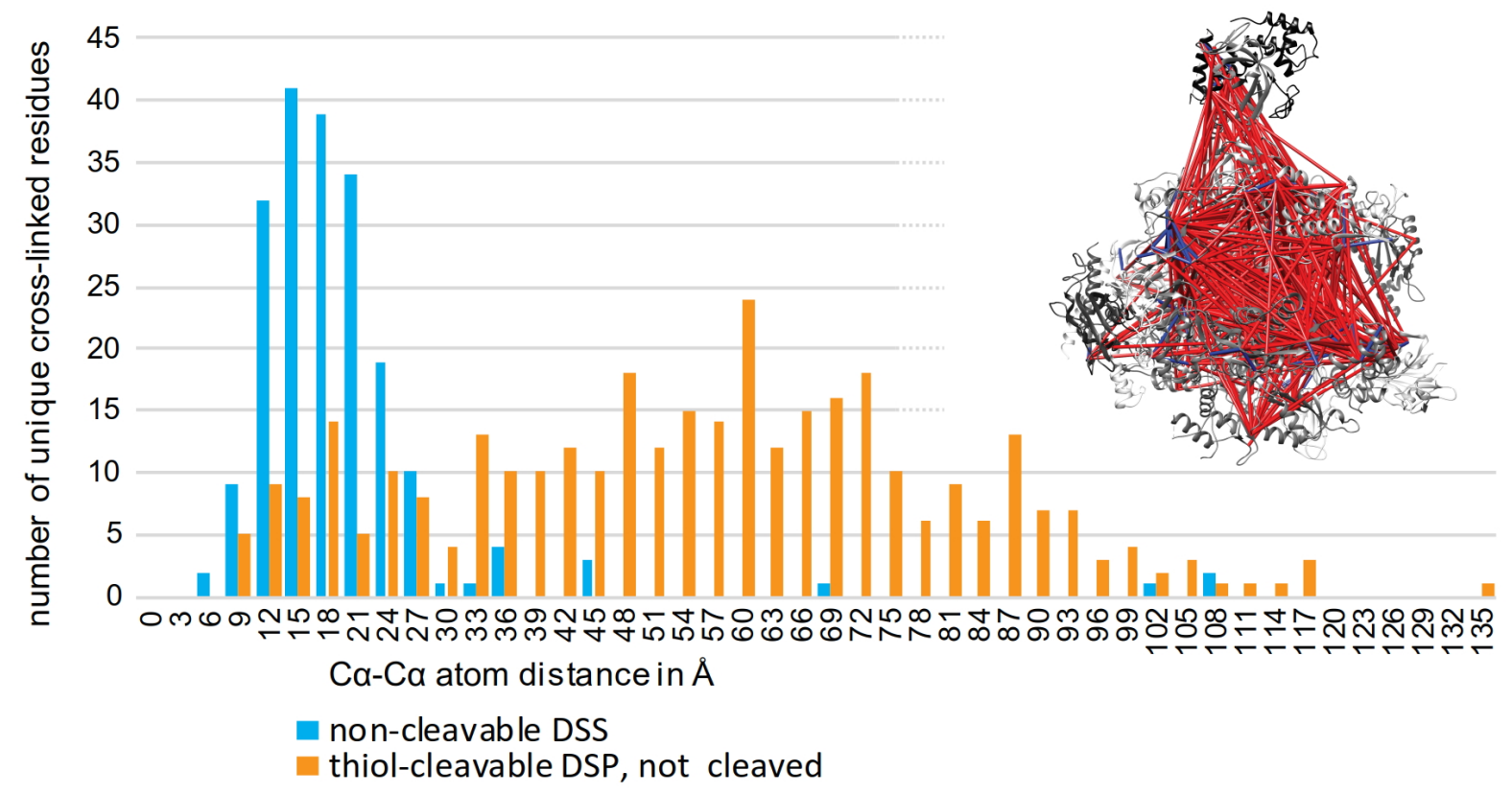

Figure 4: Comparison of DSS and DSP cross-linking without cleavage of cross-linker. Pol II ( $p d b: 50 Q M))^{125}$ was cross-linked in parallel with both cross-linkers, samples were analyzed by LC-MS and searched against the respective protein sequences. Identified cross-linker distances were measured on the crystal structure and were plotted as histogram. DSP cross-links are exemplary shown on the Pol II crystal structure (grey to black). Cross-link distances greater than $30 \AA$ are considered overlength and are depicted in red.

Overall, both approaches identify cross-links that fit to the respective crystal structure with a similar distance distribution and high overlap. Cross-linked peptides that are not identified by the peptidefocused approach are either characterized by a low pLink score or the peptide candidates are not present in the peptide database. 


\subsection{Application of the peptide-focused approach on cross-linked bacteria}

\subsubsection{Performance comparison of the conventional and peptide-focused approach}

The peptide-focused database search approach was benchmarked on purified cross-linked complexes and it was shown that the results obtained with the approach are similar to the conventional approach. Next, the approach was applied on cross-linked bacteria to test its performance on more complex samples. Bacteria have the largest proteomes that current algorithms for non-cleavable cross-linkers can evaluate in a reasonable time frame $\mathrm{e}^{41,140}$.

Bacillus subtilis and cereus were grown in LB medium and cross-linked in vivo during exponential growth phase without prior cell disruption. After XL-M S analysis, a conventional and a peptide-focused database search approach was applied in comparison to each other. Escherichia coli was also employed for in vivo cross-linking, but the migration pattern of DSS and DSP cross-linked proteins differed in SDSPAGE (data not shown). It was hypothesized that the outer membrane of gram-negative bacteria interferes with cross-linker diffusion into the cell, which was also described in the past ${ }^{141}$.

Table 7 summarizes the identifications obtained with both approaches. The proteomes of the two species were in silico digested and peptides with a lysine within the sequence besides the C-terminus were considered. These peptides constitute the cross-linking database search space of theoretically cross-linkable peptides. Bacillus subtilis and cereus contain 202,109 and 246,013 theoretically crosslinkable peptides. The generated peptide databases were much smaller in comparison but are based on experimental data (table 7). Nonetheless, peptide databases covered 60 to $73 \%$ of the reference proteome with at least one peptide per protein, which approximately corresponded to the expressed proteome for bacteria during exponential growth ${ }^{142}$. Proportional to the ratio of theoretically crosslinkable peptides, the database search was five to ten times faster than the conventional approach. The number of identified CSM s, cross-linked residues and inter-protein cross-linked residues were similarly high for Bacillus subtilis for both approaches. However, the peptide-focused approach outperformed the conventional approach for Bacillus cereus. Approximately 9,000 more CSM s, 200 more unique cross-linked residues and 62 more inter-protein cross-linked residues were identified with the peptide-focused approach. Bacillus cereus has approximately 1,000 protein sequences more than Bacillus subtilis. Hence, approximately 50,000 additional theoretically cross-linkable peptides need to be considered for the database search space.

By narrowing down the search space to peptides that were proven cross-link candidates in a parallel experiment, it was possible to obtain similar or more identified CSM s and cross-linked residues for complex samples like bacteria in a much shorter analysis time. 
Table 7: Comparison of a conventional and peptide-focused database approach applied on Bacillus subtilis and cereus. Cells were processed as described in chapter 2.2.3. Data was searched with pLink 1 against the reference proteome (conventional approach) and against a peptide database generated in parallel.

\begin{tabular}{rcc}
\hline Badillussubtilis (4,260 proteins) & Conventional approach & Peptide-focused approach \\
\hline Theoretically cross-linkable peptides & 202,109 & 29,689 \\
Proteins covered in database & 4,260 & 3,115 \\
Identified CSMs & 12,786 & 14,187 \\
Identified unique cross-linked residues & 1,197 & 1,167 \\
Inter-protein cross-linked residues & 271 & 262 \\
Relative average search time & $100 \%$ & $18.18 \%$ \\
\hline Badilluscereus (5,240 proteins) & & \\
\hline Theoretically cross-linkable peptides & 246,013 & 20,337 \\
Proteins covered in database & 5,240 & 3,103 \\
Identified CSMs & 13,909 & 22,950 \\
Identified unique cross-linked residues & 884 & 1,077 \\
Inter-protein cross-linked residues & 200 & 262 \\
Relative average search time & $100 \%$ & $10.77 \%$ \\
\hline
\end{tabular}

\subsubsection{Ribosomal interaction surfaces of EF-Tu}

In vivo cross-linking of bacteria as a benchmarking experiment has shown that the peptide-focused approach yielded similar or more identified cross-linked residues than the conventional approach. Cross-linked residues identified with the peptide-focused approach were subsequently manually inspected without a fixed CSM or pLink score cut-off. Inter-protein cross-links were evaluated whether an identification was described in the past or made sense in terms of biological function. A map of protein interactions based on cross-links in exponentially growing bacilli was obtained (supplemental figure 2). Most cross-links involved the ribosome and RNA polymerase. Their dominance makes sense, since biomass production is a focus of bacteria during exponential growth phase. After all, these complexes belong to the most abundant proteins in the cell. Cross-links within multimeric complexes of the tricarboxylic acid cycle like pyruvate dehydrogenase and succinate-CoA ligase, and the aspartyl/glutamyl-tRNA amidotransferase were identified in both species (supplemental figure 2).

In addition, numerous hitherto unknown cross-linked protein interactions were identified (supplementary table 1). Exemplarily, cross-links obtained for elongation factor Tu (EF-Tu) to the ribosome were highlighted (figure 5). A model structure from E. coli is shown in figure 5, because there is no structure available for Bacilli that includes EF-Tu. The positioning of ribosomal subunits in the ribosome is identical for Bacillus subtilis, cereus and Escherichia coli. EF-Tu served as a central interaction node in the interaction map of both bacilli species. Cross-links to ribosomal proteins RL14, RL17, and RL19 were either in cross-link distance or were located very close to EF-Tu bound to the tRNA in the aminoacyl site. Therefore, they could have been cross-linked together when EF-Tu was approaching the ribosome. Surprisingly, several cross-links of EF-Tu to ribosomal proteins RS5, RS8, RS9, and RS10 of the small subunit were identified, which mostly cluster on one surface (figure 5 , central right view of the ribosome). These cross-links are not explainable by the function of EF-Tu delivering aminoacyl-tRNAs to the A-site. Some cross-links to e.g. RS9 and RS10 could be disregarded as a singular cross-linked residue, but especially RS5 and RS8 exhibited three and two unique crosslinked residues, respectively, and cross-links to RS8 and RS9 were identified in both species. The crosslinks indicated that EF-Tu might also bind at the bottom side of the ribosome and fulfil a so far unknown function. 

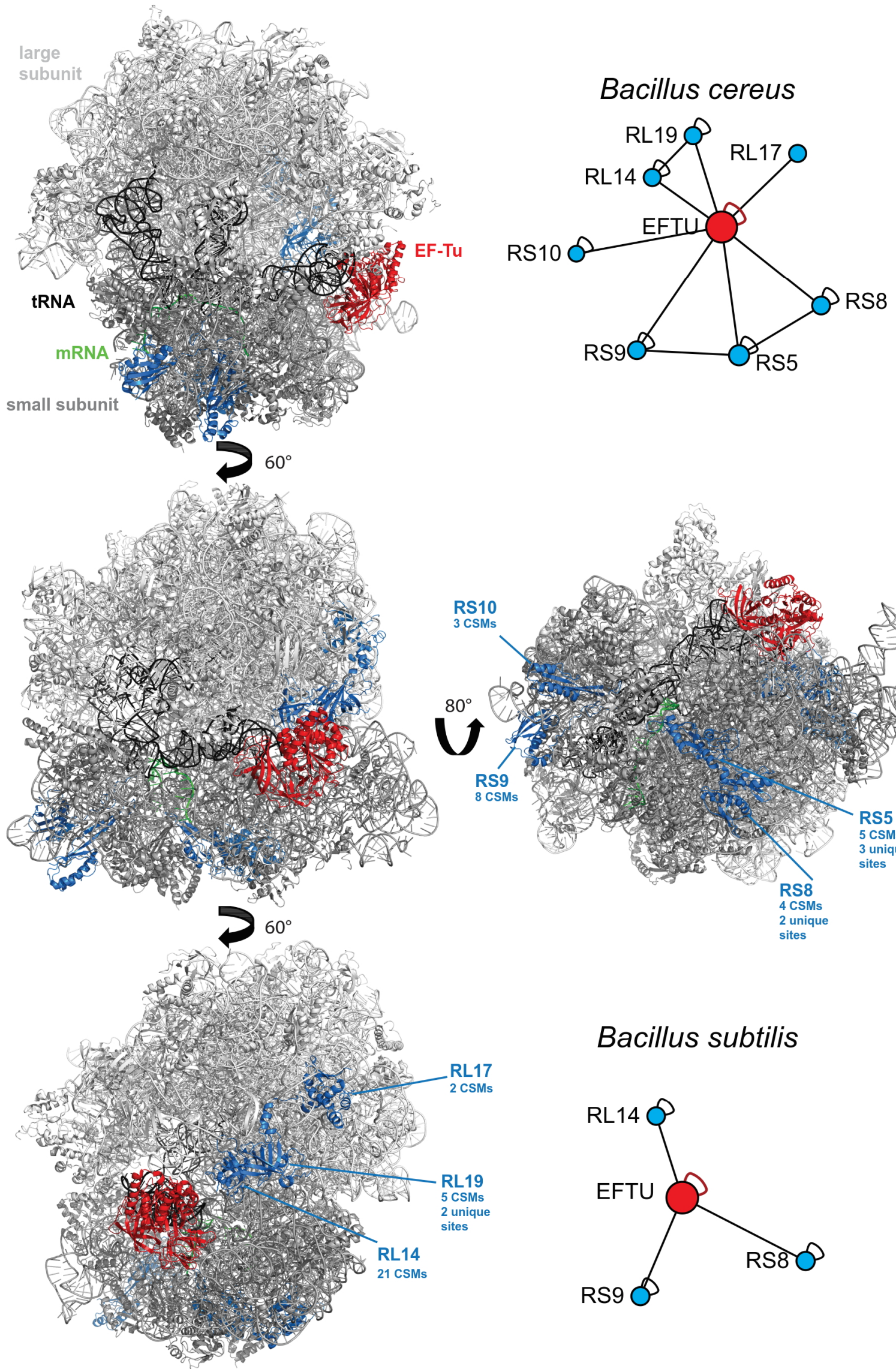

Figure 5: Interaction surfaces of EF-Tu on the ribosome. Bacteria were treated as described in chapter 2.2.3. Cross-links from EF-Tu (red circle and cartoon) to the ribosome (pdb: 5UYK, from E. coli) ${ }^{143}$ are shown as interaction maps in the bottom left and right corner. Those ribosomal subunits that cross-linked to EF-Tu are colored blue in the crystal structure. The number of identified cross-link spectral matches (CSMs) and unique linked sites is stated for each subunit. 


\subsection{XL-M S analysis of synaptosomes}

\subsubsection{M odifications of the synaptosome preparation protocol}

Once the peptide-focused approach was established on purified cross-linked complexes and crosslinked bacteria, it was possible to apply it on the actual sample of interest: purified synaptosomes. However, synaptosomes are not inherently compact and stable compartments but are generated from synapses by mechanic shearing during brain homogenization and subsequent enrichment ${ }^{119}$. Synaptosome preparations are therefore substantially contaminated with proteins and membranes of non-synaptic origin. In addition, synaptosomes contain mitochondria that tend to dominate proteomic experiments of synapses. Cross-linking mass spectrometry analysis of complex samples is currently mainly limited to the most abundant proteins. Cross-linked peptides are competing for fragmentation in a data-dependent LC-MS acquisition and they are a minority among linear peptides. Hence, it was crucial to obtain a clean synaptosome fraction without contaminant proteins and to deplete mitochondrial proteins after cross-linking of interacting synaptic components by cross-linking.

\subsubsection{Depletion of myelin fragments by continuous sucrose gradient centrifugation}

Myelin fragments are common extracellular contaminants in synaptosome preparations that comigrate with synaptosomes to some extent. Since myelin is not a functional part of synaptosomes, its depletion was desirable.

After Ficoll-based synaptosome preparation, samples were submitted to an additional continuous sucrose gradient centrifugation (chapter 2.2.4.2). Aliquots were taken before and after the depletion step and analyzed by LC-MS based proteomics. Label-free quantification could visualize how the synaptosomal protein composition changed after an additional enrichment step. As shown in figure 6, the proteome was mostly unchanged and only few proteins were significantly depleted after continuous sucrose gradient centrifugation. M ost prominently depleted proteins were myelin proteins (orange), while depletion of histones (blue) and hemoglobin (red) was observed as well. The depletion also affected the subsequent cross-linking analysis. In a follow-up experiment, synaptosomal preparation was either terminated at the step of discontinuous Ficoll gradient centrifugation (chapter 2.2.4.1) or extended by an additional 25 - $55 \%$ continuous sucrose gradient centrifugation (chapter 2.2.4.2). Samples from either purification strategy were cross-linked and cross-linking products were analyzed by LC-M S (chapter 2.2.4.4 and 2.3). The Ficoll-only sample led to the identification of 2,751 CSM s attributed to the myelin proteins colored in orange in figure 6 , which corresponds to $7.9 \%$ of all identified CSM s in that sample. In contrast, the additional centrifugation step decreased their preeminence in two independent samples to 543 and 640 CSM s corresponding to $1.3 \%$ and $1.6 \%$ of all identified CSM s, respectively. However, 2,3-cyclic-nucleotide 3-phosphodiesterase was not as strongly depleted as myelin basic and proteolipid protein. The phosphodiesterase is lysine-rich and accounted for 75 to $80 \%$ of identified CSM s among myelin-associated proteins Overall, an additional continuous sucrose gradient centrifugation step led to cleaner synaptosomes. The latter were still metabolically 
active according to a glutamate release assay performed after each preparation (data not shown). Thereby, a subsequent cross-linking analysis could have a stronger focus on synaptic proteins. Dr. Momchil Ninov (Bioanalytical Mass Spectrometry, Max-Planck-Institute for Biophysical Chemistry, Göttingen, Germany) performed all continuous sucrose gradient centrifugations.

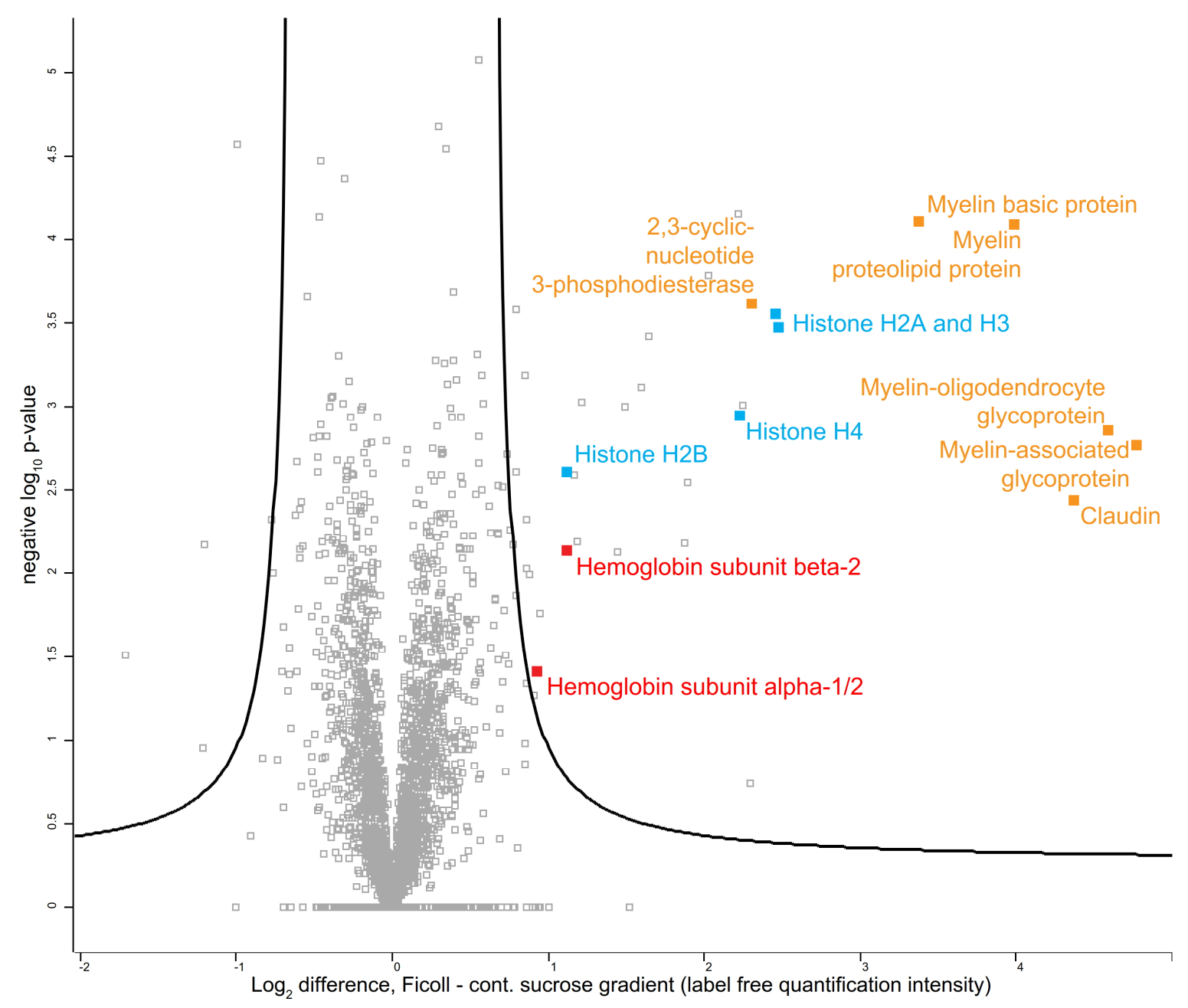

Figure 6: Scatter plot of fold changes of synaptosomes prepared with discontinuous Ficoll gradient and an additional continuous sucrose gradient plotted against the negative $\log _{10} \mathrm{p}$-value (volcano plot). Three independent bioreplicates were prepared as described in chapter 2.2.4.1 and 2.2.4.2. Data analysis was performed as described in chapter 2.4.1. See text for details.

\subsubsection{Depletion of mitochondrial proteins}

An additional sucrose gradient centrifugation step was able to deplete extra-synaptosomal myelin protein contamination. In contrast, mitochondria are a common source for contaminant proteins that are of extra- as well as intra-synaptosomal origin. Therefore, a depletion of intra-synaptosomal mitochondria after lysis of synaptosomes was pursued. 
After synaptosome preparation (chapter 2.2.4.1) without myelin depletion, synaptosomes were crosslinked to freeze near-native interactions (chapter 2.2.4.4) followed by lysis. Subsequently, a protocol was set up to deplete mitochondria prior to LC-M S analysis.

A discontinuous sucrose gradient centrifugation protocol was chosen as a starting point, which was suitable to enrich synaptosomes as well as synaptosomal plasma membranes after osmotic lysis (figure 7A, left icon). Both have similar buoyant densities in sucrose, i.e. they have comparable migration properties in density centrifugation, and should enrich at the interface of the $1 \mathrm{M}$ and $1.2 \mathrm{M}$ sucrose layer ${ }^{123}$. Synaptosomes were cross-linked, lysed, and fractionated by discontinuous sucrose gradient. The interface between $1 \mathrm{M}$ and $1.2 \mathrm{M}$ sucrose that supposedly contains synaptosomal membranes and the pellet below the 1.2 M sucrose layer were collected. Similar to chapter 3.4.1.1, the effect on protein composition was monitored by LC-MS based label-free quantification of linear, non-crosslinked peptides in comparison to a control sample that was not lysed and fractionated (figure 7A). The pellet fraction was enriched for mitochondrial and depleted for synaptic proteins (figure 7A, right), while the layer on top of $1.2 \mathrm{M}$ sucrose equally contained mitochondrial as well as synaptic proteins (figure 7A, center).

The mitochondrial contamination of the $1.2 \mathrm{M}$ layer, which should have been devoid or depleted of mitochondrial proteins, was possibly due to the cross-linking reaction changing the migration behavior of different membranes and organelles. Thus, the gradient was modified to deplete mitochondria in a cross-linked sample. Since it was not aimed to enrich or deplete other synaptic sub-fractions like free synaptic vesicles, the $0.85 \mathrm{M}$ and $1 \mathrm{M}$ sucrose layers were omitted in favor of a single sucrose cushion. Different sucrose concentrations were prepared for centrifugation in parallel and the effect on mitochondrial proteins was analyzed by LC-MS (Figure 7B). With decreasing sucrose concentration of the cushion, the bulk of mitochondrial proteins was increasingly less abundant in the fraction as compared to a non-fractionated control. The depletion of mitochondrial proteins was visible as a deviation from the $y=x$ diagonal. The use of a $1.05 \mathrm{M}$ sucrose cushion led to a two- to four-fold depletion of mitochondrial proteins on average, while the abundance of synaptic proteins did not change. Notably, the protein amount also decreased proportionally and necessitated a scale-up to obtain enough material for subsequent LC-M S analysis. 


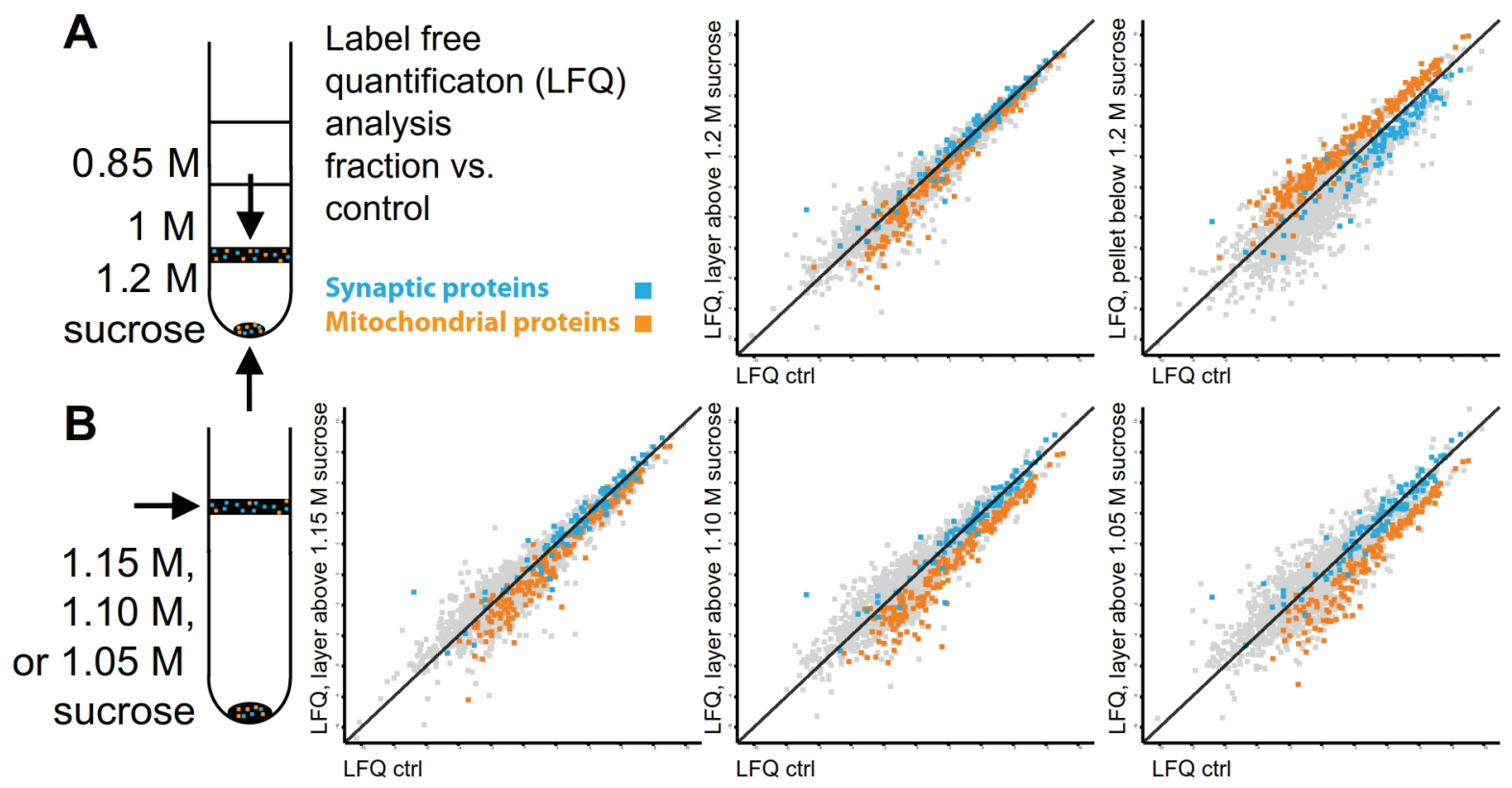

Figure 7: Proteomics label-free quantification analysis of synaptosomal plasma membrane preparations with a reported 123 (A) and an adapted protocol (B). The icons on the left show the formulation of the discontinuous sucrose gradient layers with arrows indicating the respective analyzed sample. A: scatter plots of the $1.2 \mathrm{M}$ layer and pellet of a regular discontinuous sucrose gradient $\mathbf{1 2 3}$ in comparison to a control. B: scatter plots of fractions obtained from different sucrose concentrations as single cushion on top of which the sample was carefully placed and centrifuged. M itochondrial proteins were decreasing in abundance with decreasing sucrose concentration.

The reproducibility of mitochondria depletion was assessed by preparing three bioreplicate synaptosome preparations (chapter 2.2.4.1 and 2.2.4.4), LC-M S and statistical analysis (chapter 2.4.1 and 2.4.5). Utilizing a modified discontinuous sucrose gradient with a $1.05 \mathrm{M}$ sucrose cushion led to a significant depletion of mitochondrial and significant enrichment of synaptic proteins on average (figure 8). In total, 2,111 proteins were quantified, of which 479 were significantly enriched and 521 were significantly depleted (supplementary table 2). Exemplary representatives for synaptic vesicles, the active zone, endocytosis-related proteins, and plasma membrane channels were enriched or did not change in their abundance. Interestingly, presynaptic active zone scaffold proteins like piccolo, bassoon and ERC2 were significantly depleted, while others, i.e. Cask and Liprin-alpha-4, did not change in their abundance. Likewise, complexin 1 and 2 were less abundant after enrichment, while SNARE proteins, synaptotagmin, and other factors involved in synaptic vesicle fusion were enriched or have not changed in abundance. 


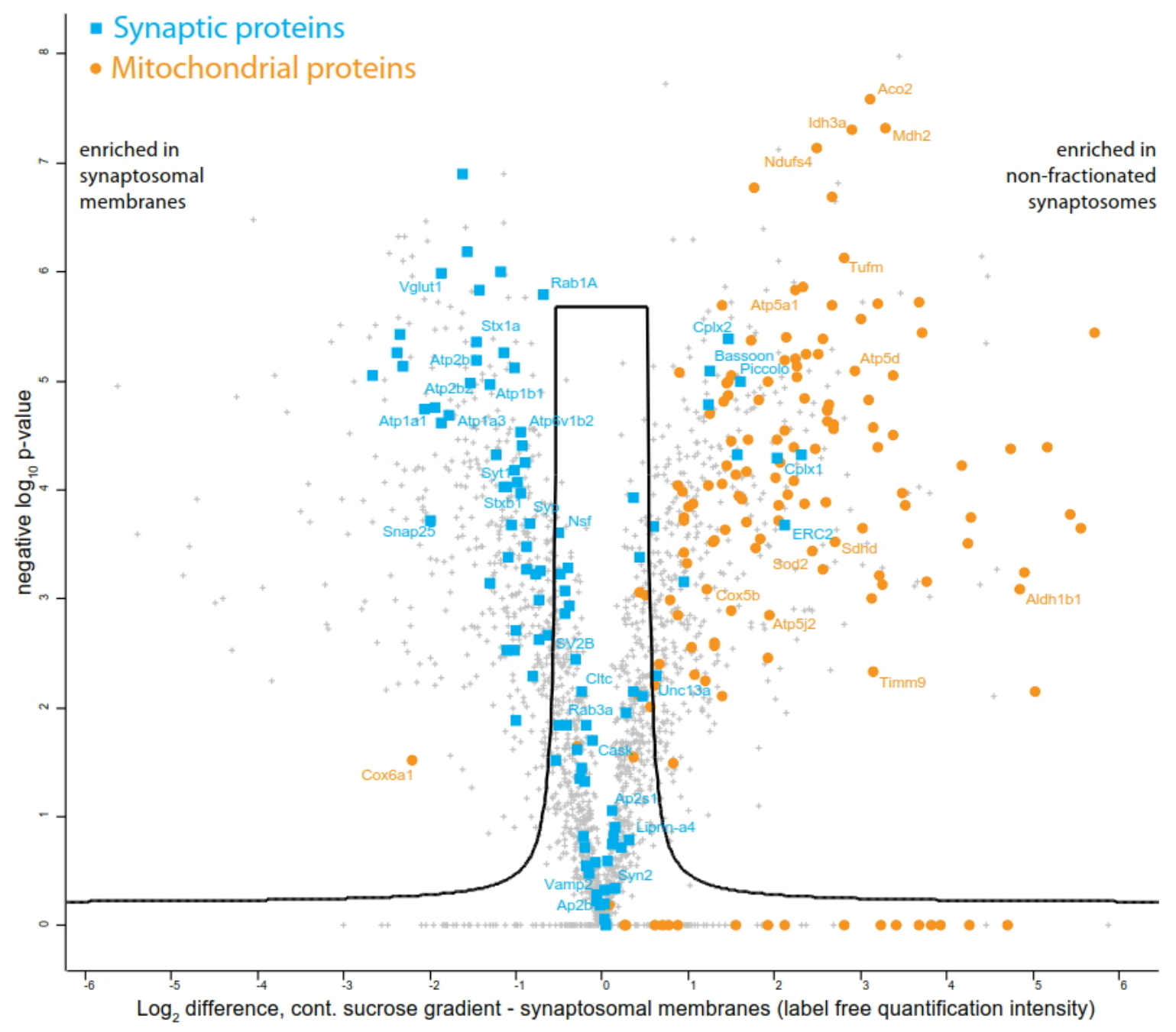

Figure 8: Scatter plot of fold changes of synaptosomes prepared by Ficoll and continuous sucrose gradient in comparison to subsequent lysis and mitochondria depletion plotted against the negative $\log _{10} p$-value (volcano plot). Three independent bioreplicates were prepared as described in chapter 2.2.4.1 to 2.2.4.5. Data analysis was performed as described in chapter 2.4.1 and 2.4.5. Some proteins of mitochondrial (orange) and synaptic (blue) origin were labelled as an example. The majority of synaptic proteins did not change or were significantly enriched after lysis and centrifugation, while the contrary was true for mitochondrial proteins.

Similar to myelin fragment removal, the depletion of mitochondrial proteins had an effect on subsequent cross-linking analysis. Without a depletion step, approximately $30 \%$ of all identified CSM s were within and between mitochondrial proteins. Including a mitochondria depletion step, the proportion of mitochondria-related CSMs dropped to $8 \%$, which was proportional to the depletion factor observed in the LFQ analysis. Thus, the removal of mitochondrial proteins enabled a stronger focus on proteins involved in synaptic functions. 


\subsubsection{Strong cation exchange chromatography as an additional method for cross-linked peptide fractionation}

After the establishment of two biochemical workflows for the depletion of myelin and mitochondrial contaminant proteins, the next focus was the improvement of the detection of cross-linked peptides in LC-MS.

Current methods for cross-linked peptide enrichment were developed for moderately large protein samples, like purified multimeric complexes ${ }^{30}$. Cell and organelle cross-linking samples are several orders of magnitude more complex and would therefore profit from a more elaborate fractionation procedure. Since peptide size exclusion (pSEC) ${ }^{30,133}$ and strong cation exchange chromatography $(\mathrm{SCX})^{34,132}$ are popular methods for the enrichment of cross-linked peptides, it was reasonable to combine both.

Three cross-linking reaction replicates were processed in parallel. Two of them yielded approximately 200 to $250 \mu \mathrm{g}$ of protein after mitochondria depletion, which was the minimum amount necessary for pSEC and obtaining satisfactory cross-linking identification results according to personal experience. The third sample yielded $450 \mu \mathrm{g}$ and was therefore split by SCX to two fractions prior to PSEC to assess the gain in identifications that is possible by more extensive fractionation (chapter 2.3.4). Indeed, an accessory fractionation step by SCX led to more identifications in total and more cross-links unique to the respective sample (figure 9 , blue circle). The high salt fraction from the additional SCX fractionation exhibited higher charge states and identified precursors were larger on average in comparison to a sample fractionated by pSEC only (data not shown). An additional SCX peptide fractionation step prior to PSEC necessitates a larger starting amount of sample, though, which could represent a challenge for certain samples, e.g. synaptosomal plasma membranes after mitochondria depletion. Given a reproducible availability of larger amounts of cross-linked synaptosomal plasma membranes, e.g. 0.5 to $1 \mathrm{mg}$ of protein after mitochondria depletion, SCX would be an effective method in addition and prior to SEC for a deeper fractionation.

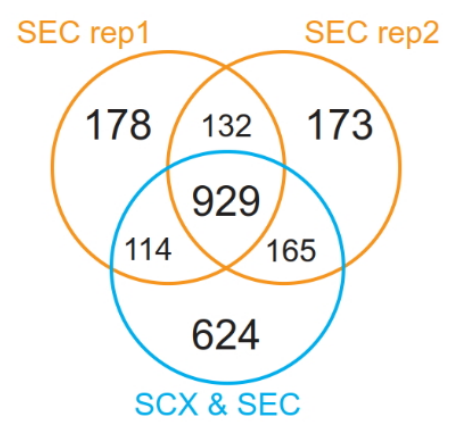

Figure 9: Venn diagram comparing unique cross-links identified from differentially fractionated replicates of cross-linked synaptosomes. Three cross-linking reaction replicates of synaptosomes were digested, and two were fractionated by peptide size exclusion chromatography (SEC) prior to LC-MS analysis (chapter 2.3.5). The third was additionally fractionated in two fractions by polymeric strong cation exchange chromatography (SCX, chapter2.3.4) before peptide size exclusion and LCMS analysis. 


\subsubsection{Application of a peptide-focused approach on rat brain synaptosomes}

The peptide-focused approach was established and benchmarked on purified cross-linked complexes and cross-linked bacteria. After optimizing the protocol for synaptosome preparation including myelin and mitochondria depletion, the peptide-focused approach was evaluated for cross-linked synaptosomes.

Therefore, a peptide database of cross-linked synaptosomes was generated and the approach was compared with the conventional approach of searching against the entire proteome of rattus norvegicus (table 8). pLink 1 was not able to process a database search against a mammalian proteome in a reasonable time frame, thus Kojak was used for the following comparison ${ }^{42}$. An in silico digestion of the rattus norvegicus proteome and a consideration of peptides with a lysine within the sequence besides the C-terminus leads to 1,481,335 theoretically cross-linkable peptides as the peptide candidate search space. In contrast, the search space of the peptide-focused approach comprised only 35,604 peptides, which is $2.4 \%$ compared to the entire cross-linkable peptide proteome. The peptide database contained peptides referring to 12,280 proteins, which corresponded to a coverage of $41.8 \%$ of the Rattus norvegicus uniprot reference proteome (29,351 proteins). Accordingly, the search took less than one twelfth of the time. At the same time, it was able to identify one and a half times as much CSM s and unique cross-linked residues.

During the course of the present study, a novel version of the cross-linking identification software pLink was made publicly available, i.e. pLink 2 , but was not published in a peer-reviewed journal, yet. It is much faster and, according to the developers, has an improved precision. With pLink 2 , the peptidefocused approach only identified $80 \%$ of CSMs in comparison with a search against the entire proteome. However, it took $3.36 \%$ of the search time and identified $51 \%$ more inter-protein CSM s and $22 \%$ more unique inter-protein cross-linked residues. However, the comparison with plink 2 needs to be regarded with suspicion, since the new algorithm is not published, yet, and its mode of operation is not known. The comparison made here therefore could have been set up incorrectly.

In conclusion, a peptide-focused approach was faster than a conventional search and identified more cross-links and inter-links, respectively, depending on the search engine.

Table 8: Comparison of a conventional and peptide-focused database approach applied on rattus norvegicus. Synaptosomes were prepared from rat brains, followed by cross-linking and synaptosomal plasma membrane enrichment. Samples were proteolytically digested and analyzed by LC-MS. Data was searched with Kojak against the reference proteome (conventional approach) and against a peptide database generated from six independent DSP cross-link and cleavage replicates. Percentages in brackets refer to results obtained with the peptide-focused approach in relation to those obtained with the conventional approach.

\begin{tabular}{rcc}
\hline Rattus nonegias (29,351 proteins) & Conventional approach & Peptide-focused approach \\
\hline Theoretically cross-linkable peptides & $1,481,335$ & 35,604 \\
Proteins covered in database & 29,351 & 12,280 \\
Identified CSM s & 14,348 & 20,770 \\
Identified unique cross-linked residues & 1,603 & 2,420 \\
Average search time & $100 \%$ & $8.15 \%$ \\
\hline
\end{tabular}

Due to the stochastic nature of cross-linking and data-dependent LC-MS acquisition and because of the limited sensitivity of mass spectrometry, it was assumed that a single dataset of cleaved and 
alkylated DSP-linked peptides would not cover all possibly cross-linkable peptides. Therefore, several datasets of DSP cross-linked, and cleaved samples were acquired and the gain in unique peptides with a cleaved DSP cross-linker was observed (figure 10). Each dataset of cross-linked and cleaved proteins derived from synaptosomal plasma membranes led to some additionally identified unique peptides, but the individual gains declined with a saturation curve approaching 37,000 peptides. A saturation of newly identified peptides with a cleaved residual cross-linker indicated that cross-linking of synaptosomal membranes was reproducible to some extent.

Therefore, the present peptide database could be considered as near complete and sufficient for the analysis of synaptosomes.

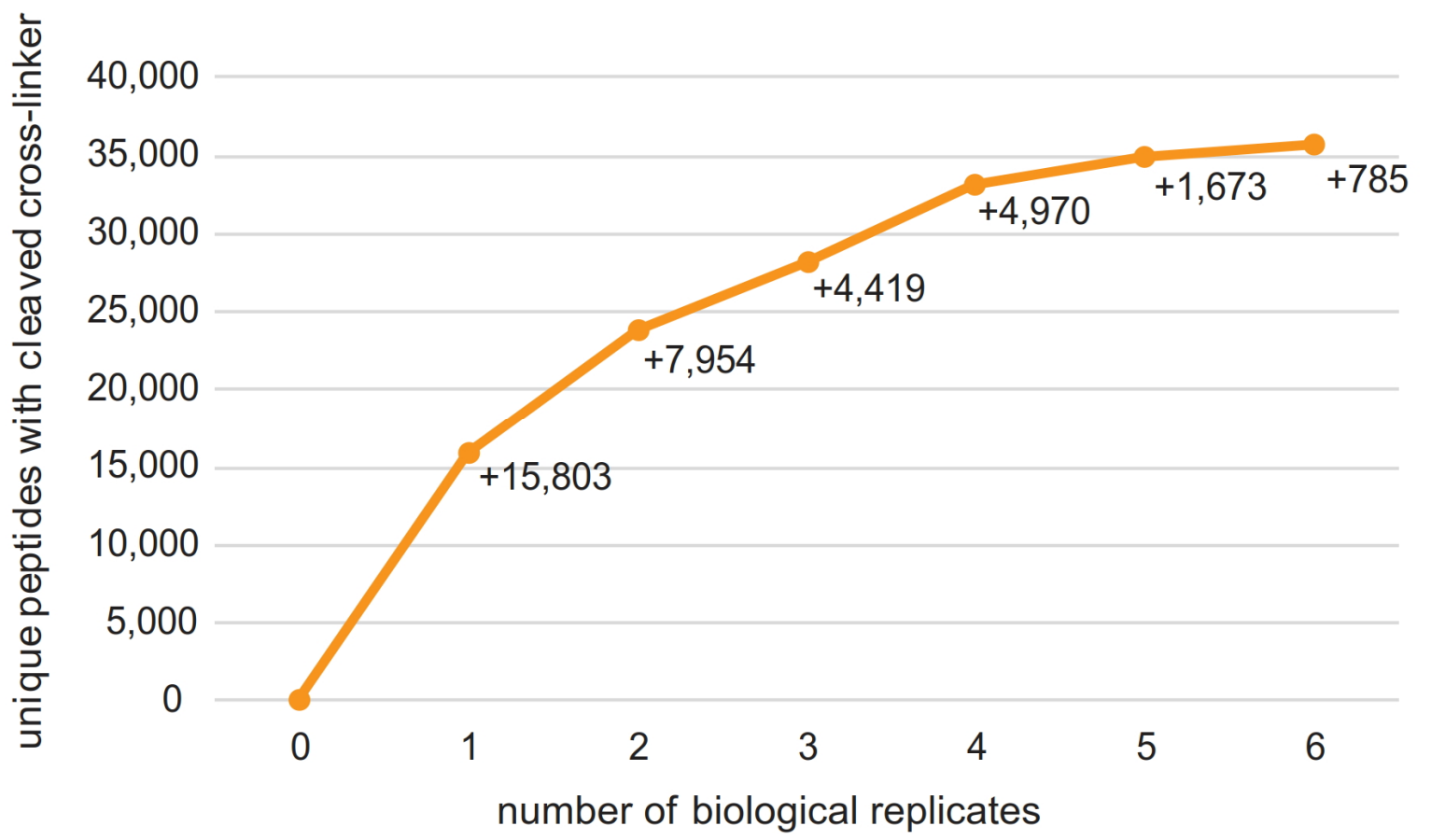

Figure 10: Gain in unique peptides per replicate for the generation of a combined peptide database. Synaptosomes were cross-linked with thiol-cleavable DSP. Cross-links were enriched after digestion, cleaved by reduction and alkylation and analyzed by LC-MS. With increasing number of biological replicates, the gain in previously unidentified peptides with a cleaved DSP-cross-linker is decreasing.

The peptide database of cross-linked synaptosomes covered approximately $42 \%$ of the rattus norvegicus reference proteome (table 8). It was examined, whether the peptide database has a bias and does not cover low abundant proteins in an LC-M S analysis of synaptosomes.

Figure 11 displays the ranking of proteins according to their abundance. High abundant proteins are on the left, while low abundant proteins are on the right side of the plot. Proteins that are represented in the peptide database (blue squares) are distributed across the entire protein abundance range.

The peptide database has no apparent bias in the representation of low abundant proteins according to the LC-MS analysis of a synaptosomes sample. The approach therefore enables the cross-linking analysis of low abundant proteins, which would be missed in a cross-linking analysis with a limited database containing only the most abundant proteins as described in chapter 1.2.2. 


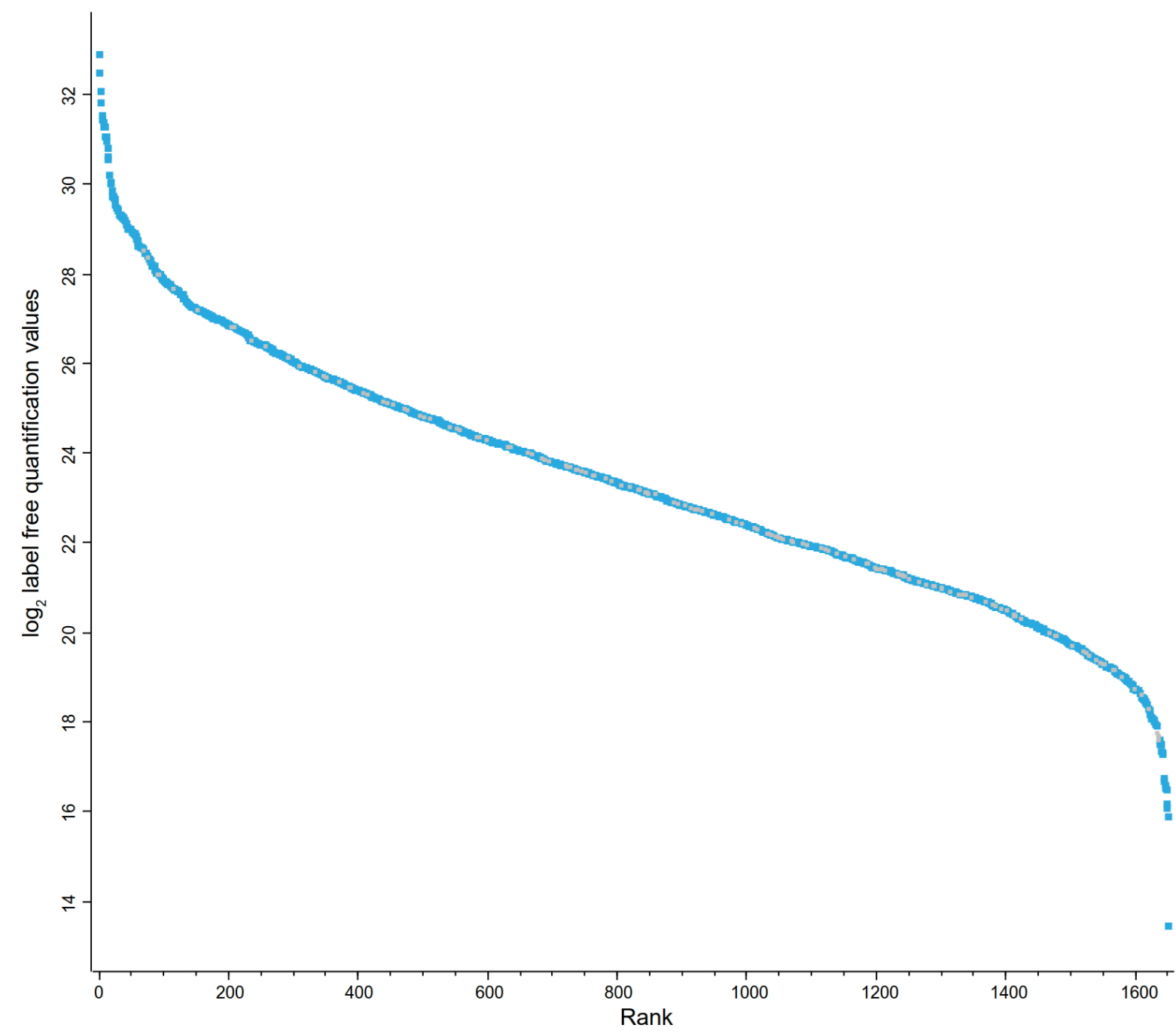

Figure 11: Distribution of proteins that are represented in the peptide database over the abundance range of the synaptosome proteome. A synaptosome sample was analyzed by label-free quantification proteomics (chapter 2.2.4.1 to 2.2.4.5). Identified proteins were ranked and plotted according to their abundance (chapter 2.4.1). Proteins that are represented in the peptide database generated for the analysis of cross-linked synaptosomes (chapter 2.4.2) are labelled blue. 


\subsubsection{The protein cross-linking interactome of rat brain synapses}

A peptide-focused approach for the XL-M S analysis of complex samples was established as well as an improved biochemical preparation for the analysis of synaptic proteins. Next, all improvements were brought together for the identification of protein interactions in the synapse by XL-M S.

Synaptosomes from rat brains were prepared and myelin fragments were depleted (chapter 2.2.4.1 and 2.2.4.2). Subsequently, synaptosomes were cross-linked under resting and excited conditions, were lysed, and mitochondria were depleted (chapter 2.2.4.4 and 2.2.4.5). After XL-M S acquisition, cross-linked residues were identified with the peptide-focused approach using pLink 1 and a peptide database consisting of 35,604 peptides (table 8 , figure 10 ) to obtain a protein interaction map (chapter 2.4.3). Overall, data from eleven cross-linking reaction replicates corresponding to four biological replicates was analyzed and combined to a total of 4,377 unique cross-linked residues (supplementary table 3). All inter-protein links were manually evaluated. No strict pLink score or CSM cut-off was applied. Due to high interconnectedness, the interaction map was split in five sub-maps of proteins not typical for synapses (supplemental figure 3), mitochondrial proteins (supplemental figure 4), synaptic proteins (figure 12), membrane receptors and channels (figure 13), and cell adhesion and cytoskeleton proteins (figure 14). Some proteins were present in multiple sub-maps, for example, gamma-actin (ACTG) was cross-linked to most of its interactors in the sub-map of cytoskeleton proteins, but also appeared cross-linked to sodium/potassium transporting ATPase subunit beta-1 (AT1B1) in the sub-map of membrane receptors and channels. Several cross-linked proteins were identified that were not specific for synapses, e.g. subunits of $20 \mathrm{~S}$ proteasome and ribosomes (supplemental figure 3). Despite the depletion efforts, myelin basic protein, myelin proteolipid protein and 2' 3 '-cyclic nucleotide 3'-phosphodiesterase were found cross-linked but were mostly identified with intra-protein cross-links. All enzymes of the glycolysis pathway were detected with numerous intra-links, next to few endoplasmic reticulum proteins and inter-protein cross-linked subunits of the TRiC chaperonin complex, $20 \mathrm{~S}$ proteasome and the ribosome. The latter two were described as common contaminants of synaptic vesicle preparations in the past ${ }^{75}$.

Although mitochondrial proteins were depleted two- to four-fold, numerous cross-links were still identified between and within proteins that were localized in the mitochondrion (supplemental figure 4). Most prominently, subunits of all complexes of the electron transport chain were detected crosslinked, which comprised roughly half of all identified mitochondrial cross-links. In addition, all enzymes of the tricarboxylic acid cycle exhibited intra-links and inter-links in case of multimeric enzymes.

According to manual evaluation, two thirds of identified cross-linked residues were valid cross-links within and between synaptic proteins. Along with cell-signaling components, figure 12 depicts proteins that are part of the active zone, reside in synaptic vesicles, or are involved in their fusion and/or recycling by endocytosis. V-type proton ATPase was the largest coherent complex found cross-linked. Alongside, several other proteins residing at the membrane of synaptic vesicles and thus in tight spatial constraints were found cross-linked to each other, e.g. synapsin isoforms, and synaptophysin, synaptoporin and synaptic vesicle glycoprotein A (SV2A). The subunits of the SNARE complex, i.e. vesicle-associated membrane protein 2 (Vamp2, synaptobrevin), Syntaxin-1 (Stx1), and synaptosomalassociated protein 25 (Snap25), were not cross-linked to each other, but to several factors involved in SNARE complex assembly, disassembly, and regulation. Syntaxin-binding protein 1 (Stxbp1/M unc181) appeared as a main hub in that matter being cross-linked to Stx1A, 1B, and synaptotagmin-1 (Syt1). Furthermore, Stxbp1/M unc18-1 was cross-linked to factors involved in synaptic vesicle recruitment, docking and retrieval by endocytosis after fusion, i.e. synaptojanin, Rab3A, and VP33B. Subunits of 
guanine-nucleotide binding proteins (e.g. Gnao, Gng) that are involved in cell signaling were mostly cross-linked among each other but interestingly, Gnao also cross-linked to Stxbp1, Nsf and a subunit of the V-type proton ATPase. In addition, Gnao cross-linked to ryanodine receptor 1 and subunit alpha2 of $\mathrm{Na}^{+} / \mathrm{K}^{+}$-transporting ATPase, while $\mathrm{Gng} 3$ (guanine nucleotide binding protein gamma-3) crosslinked to voltage-dependent anion channel 3 (VDAC) (figure 13).

The $\mathrm{Na}^{+} / \mathrm{K}^{+}$-transporting ATPase complex formed a major node in the interaction map of membrane channels and receptors as shown in figure 13. In addition to cross-links between alpha, beta and gamma subunits, there were also cross-links between alpha isoforms indicating the existence of oligomeric trimer complexes. Oligomerization was also observed for isoforms of plasma membrane calcium transporting ATPase (PM CA). A multitude of protein classes cross-linked to different subunits and isoforms of $\mathrm{Na}^{+} / \mathrm{K}^{+}$-transporting ATPase. Interestingly, pyruvate kinase, which is generating ATP by phosphoenolpyruvate cleavage, was cross-linked to this ATP-dependent ion transporter as well as to a subunit of the V-type proton ATPase (figure 12). 14-3-3 proteins and N-myc downstream-regulated gene proteins (NDRG) were cross-linked to several alpha isoforms of the $\mathrm{Na}^{+} / \mathrm{K}^{+}$-transporting ATPase and a Calcium transporting ATPase. $1433 Z$ was also cross-linked to a voltage-dependent calcium channel. Furthermore, several proteins with a cytoskeletal function, cell adhesion and scaffold proteins cross-linked to various membrane channels, e.g. spectrin alpha and beta, Ankyrin-2, clathrin, and actin. Voltage-dependent anion channels are depicted among mitochondrial proteins in supplemental figure 4 as well as in figure 13, because there is evidence for their expression in the plasma membrane besides the mitochondrial outer membrane ${ }^{144}$. Several ionotropic and metabotropic glutamate and GABA receptors were identified with intra- and inter-links between oligomeric receptor subunits, but did not cross-link to other protein classes, for example guanine nucleotide-binding proteins.

Apart from cross-links to membrane channels, cytoskeletal proteins also displayed numerous crosslinks among each other and to regulators of cytoskeleton assembly and disassembly (figure 14). Gamma-actin was the main node for cytoskeleton-related protein interactions exhibiting cross-links to known actin binders like subunits of the actin-related protein (Arp) complex, F-actin capping proteins and regulators of actin polymerization, e.g. WDR1, and twinfilin-1. Other sub-clusters were formed by septins, spectrins, tubulins, 14-3-3 proteins and cell adhesion proteins assembled around Contactin-1. Chaperones formed a cluster around Hsp7c and are depicted in figure 14 because of a cross-link to a 14-3-3 protein, although not all of the reported chaperones have a cytoplasmic or synaptic localization. In summary, XL-M S analysis of synaptosomes with the peptide-focused approach was able to capture an extensive protein-protein interaction map of the synapse. 


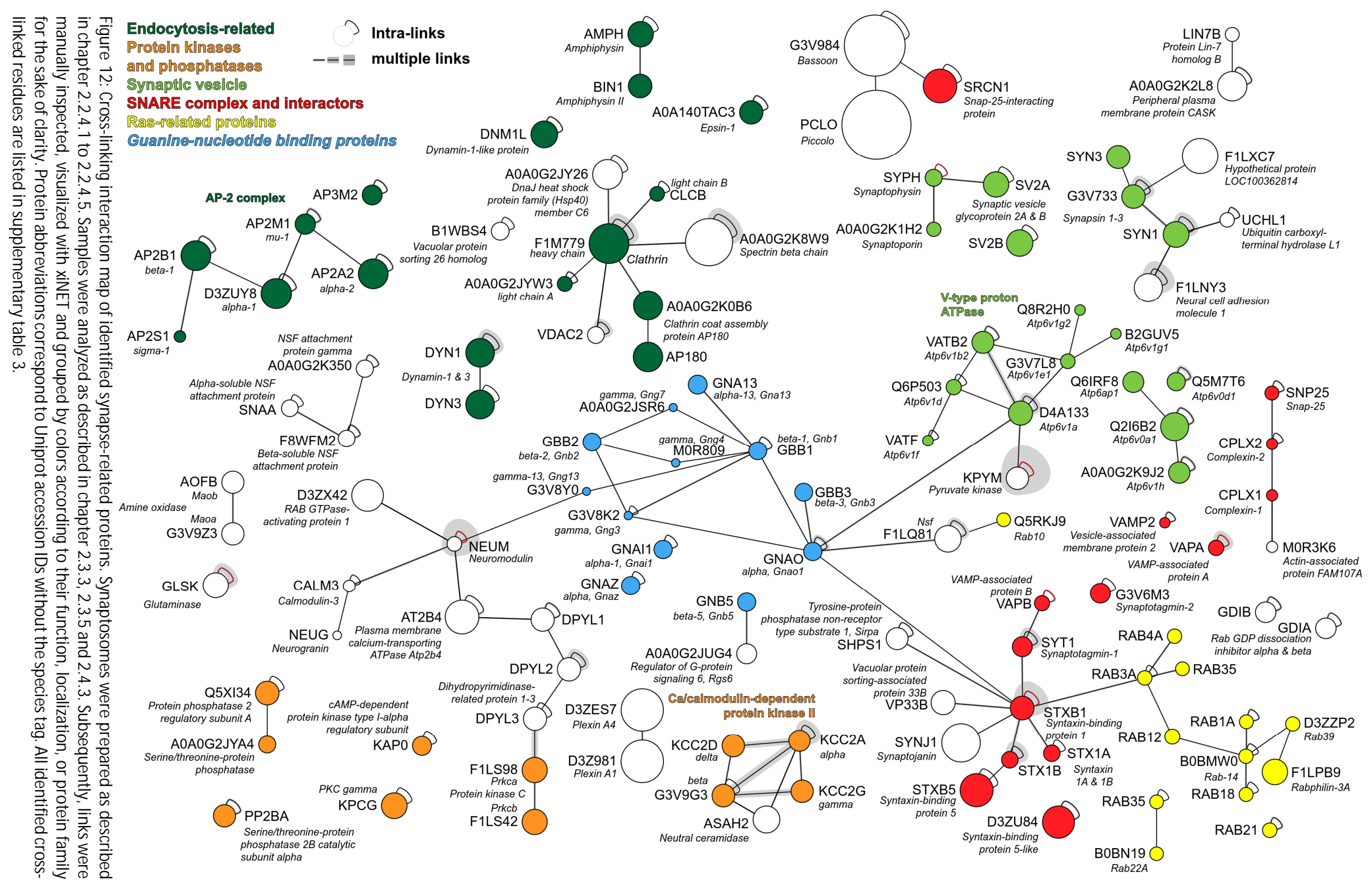


Cyrtoskeletall, cell ad|hesion \& $\quad-==$ multiple links

scaffolding proteins

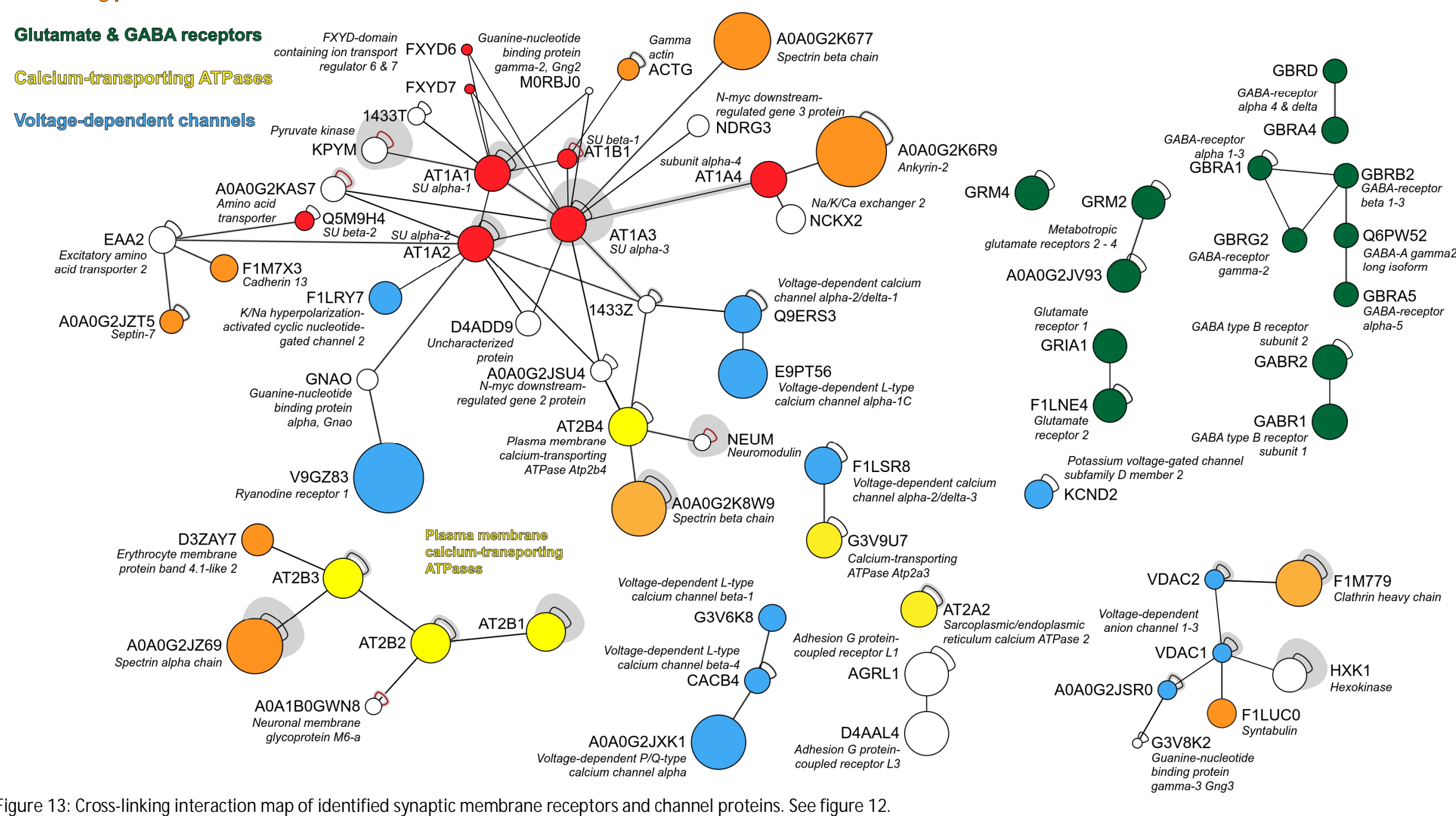

Figure 13: Cross-linking interaction map of identified synaptic membrane receptors and channel proteins. See figure 12. 
Actin-binding proteins

Spectrin-binding proteins

Hyaluronan and
proteoglycan link protein 1

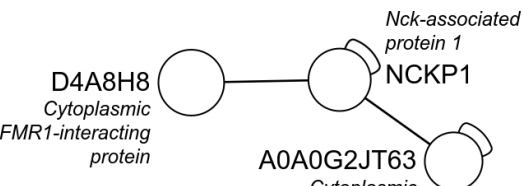

Arp 2/3 complex

ARP5L

D3ZN61

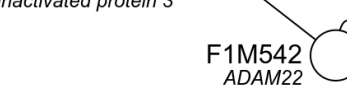

ADAM22

G3V8R2

FMR1-interacting
protein

A0A0G2JT63

subunit 4
surp
B $2 / 3272$

$\begin{array}{rr}\text { beta-3 } & \begin{array}{r}\text { Cytoplasmic } \\ \text { TBB3 }\end{array} \\ \text { FMR-interacting } \\ \text { protein 2 }\end{array}$
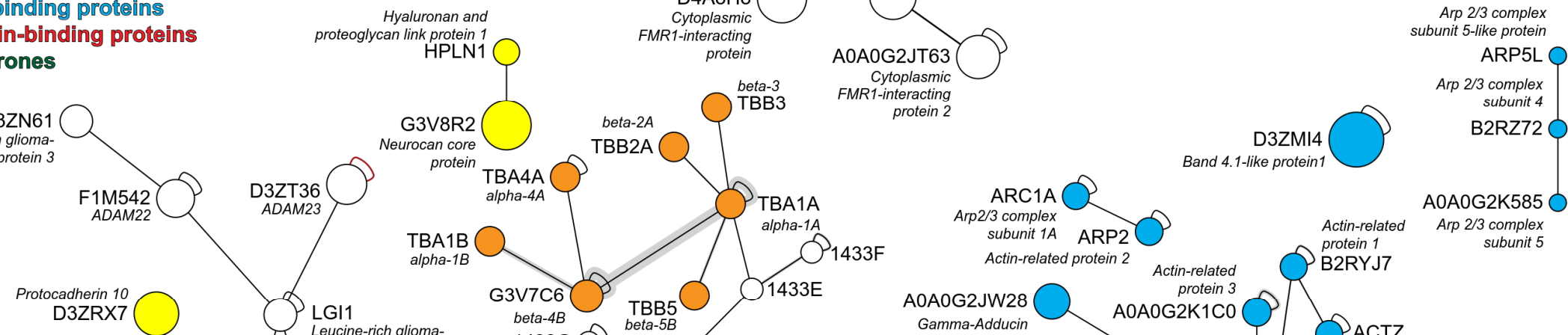

alpha-1B

$P^{\text {TBB2A }}$

Neural cell adhesion
molecule L1
A0A0G2KA95

PACN1 1O Contactin 2

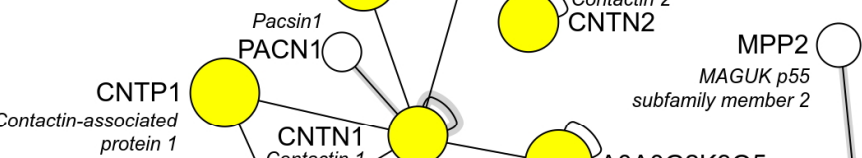

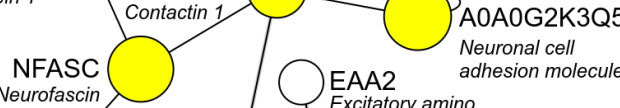

To96MJE

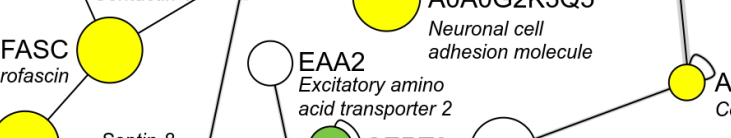

G3V9Z6 $\begin{array}{ll}\text { Stress-70 protein } & \begin{array}{c}\text { Hs70- } \\ \text { interacting protein } \\ \text { F10A1 }\end{array}\end{array}$

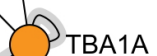

\section{TBA1A}
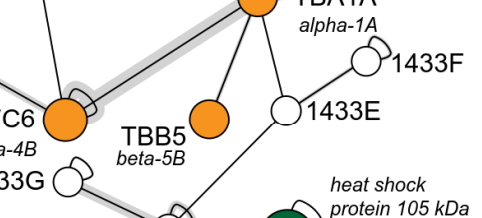

1433B/Z/T

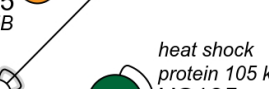

heat shock
protein $105 \mathrm{kD}$
HS105

heat shock
protein 90-alpha
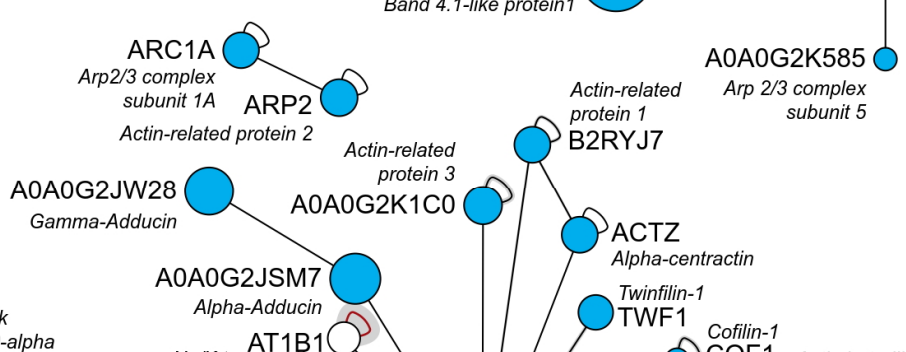

Alpha-Adducin 1 TWF1

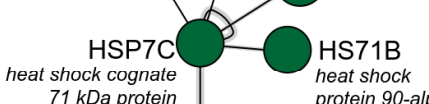

Phosphatase and

GRP75

$$
\begin{aligned}
& \text { WDR1 } \\
& \text { WD repeat } \\
& \text { domain } 19
\end{aligned}
$$
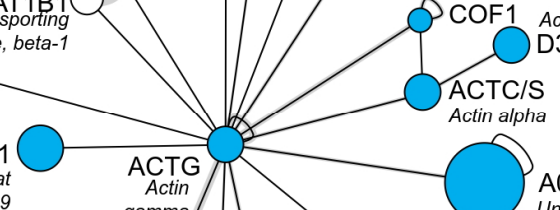

COF1 Actin beta-like

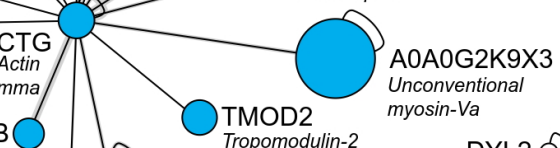
DYL2
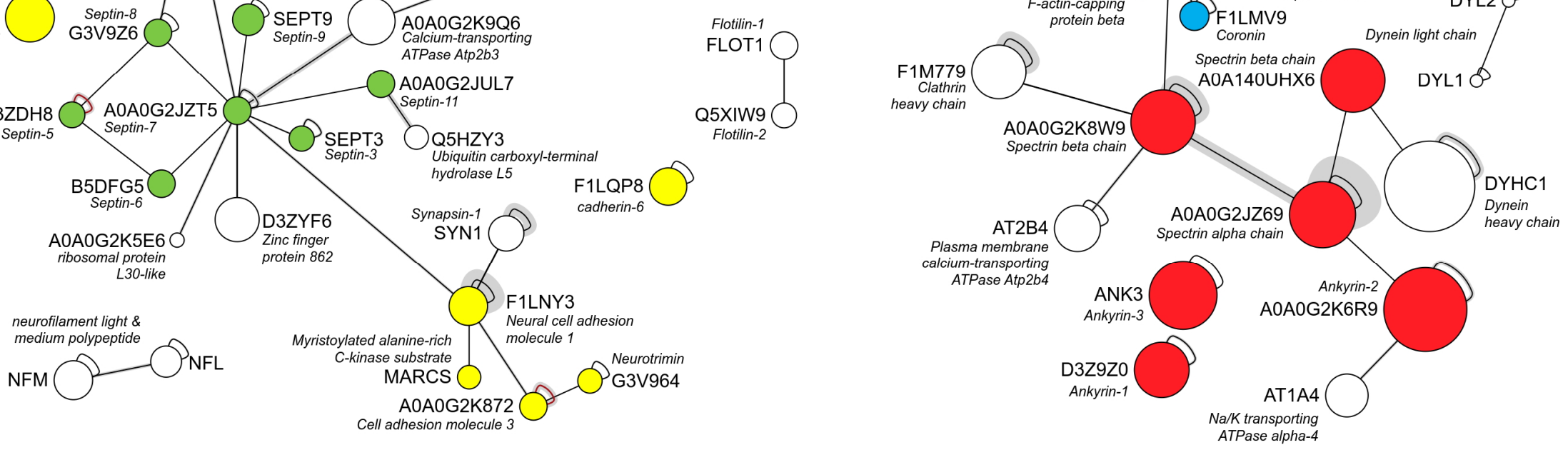



\subsubsection{Quantitative changes of cross-links in excited synaptosomes}

After establishing a static protein interaction network of synaptosomes with XL-M S, a fitting follow-up experiment was to cross-link synaptosomes under resting and excited conditions and to quantify crosslink changes in response to a conformational change. Synaptosomes are model systems for research on synapse function, because they are biochemically active and excitable compartments that release transmitters upon change of plasma membrane potential (chapter 2.2.4.3) ${ }^{119}$. The machinery, which is responsible for synapse functions like synaptic vesicle docking, priming, fusion, and recycling, is largely intact and its dynamically changing protein interactions can be studied ex vivo ${ }^{117}$. Synaptosomes depleted of contaminants were therefore cross-linked in a resting $(0.5 \mathrm{mM} \mathrm{EGTA}$ and $50 \mathrm{mM} \mathrm{KCl})$ and excited state $(1.3 \mathrm{mM} \mathrm{CaCl}, 50 \mathrm{mM} \mathrm{KCl}$ ) with isotopically labelled cross-linkers (DSS-d0 and deuterated DSS-d4, chapter 2.2.4.4). Subsequently, samples were pooled and processed jointly. XL-MS analysis was performed with the peptide-focused approach and the ratios between labels were quantified with $\mathrm{XiQ}^{1}$ (chapter 2.4.3, 2.4.4). Quantitatively changing cross-links in response to stimulation were a sign for conformational changes and changing protein interactions. In total, 3,818 unique cross-linked residues were identified and quantified from nine cross-linking reaction replicates corresponding to three biological replicates. Identified cross-linked residues were also incorporated in the interaction network in chapter 3.4.3. These nine reaction replicates included label-swapping, i.e. five stimulated synaptosome samples were cross-linked with DSS-d4 and were pooled with resting synaptosome samples cross-linked with DSS-d0 and vice versa for the other four samples. Of these cross-links, 1,848 were quantified in at least three cross-linking reaction replicates and were evaluated by a one-sample t-test. 224 cross-links had a $p$-value below 0.05 , and 129 exhibited a fold-change of at least 1.5 between excited and resting state (figure 15 and table 9). Only a minority of 24 inter-protein cross-links changed significantly, of which ten changed at least 1.5 -fold. Similar to a qualitative study focusing on identified cross-links, most cross-links changing upon excitation were quantified among the most abundant proteins.

Overall, the present study was largely able to identify changing intra-links, i.e. changes in the conformation of proteins, as a response to calcium influx and its resulting effects. Accordingly, significantly changed cross-links were quantified in calcium-binding proteins, e.g. calmodulin (Cam), synaptotagmin I (Syt1), annexin A6 (Anxa6) and CaM kinase-like vesicle-associated protein (Camkv; chapter 3.4.4.2). Because of altered ion concentration gradients across the membrane, i.e. high potassium and calcium concentrations in the buffer, and thus a changed membrane potential, many ion channels displayed significant changes. Among those were, e.g. plasma membrane and sarcoplasmic/endoplasmic reticulum calcium transporting ATPase (PM CA, SERCA), $\mathrm{Na}^{+} / \mathrm{K}^{+}$ATPase, and inositol-1,4,5-trisphosphate receptor type 1 (I3PR; chapter 3.4.4.1). Several proteins displayed significant changes that are neither reported to bind calcium ions, nor are involved in calcium signaling, but have other functions like energy metabolism (chapter 3.4.4.4), protein folding, or are located in the plasma membrane and face the extracellular side (chapter 3.4.4.3). However, several proteins, which are known to change their conformation during the events involved in synaptic vesicle exo- and endocytosis, did not exhibit significantly changed cross-links, e.g. Stx1 and Stxbp1 heterodimer ${ }^{88}$, adaptor protein 2 (AP-2) complex ${ }^{145}$, and voltage-dependent calcium channels (chapter3.4.4.5).

Since the observed changing cross-links are a consequence of structural changes that are highly individual for the respective proteins, significant cross-links are partially discussed in the following chapters. In conclusion, the calcium/potassium stimulation of synaptosomes led to significant structural effects for a wide variety of proteins in the synaptosome. 


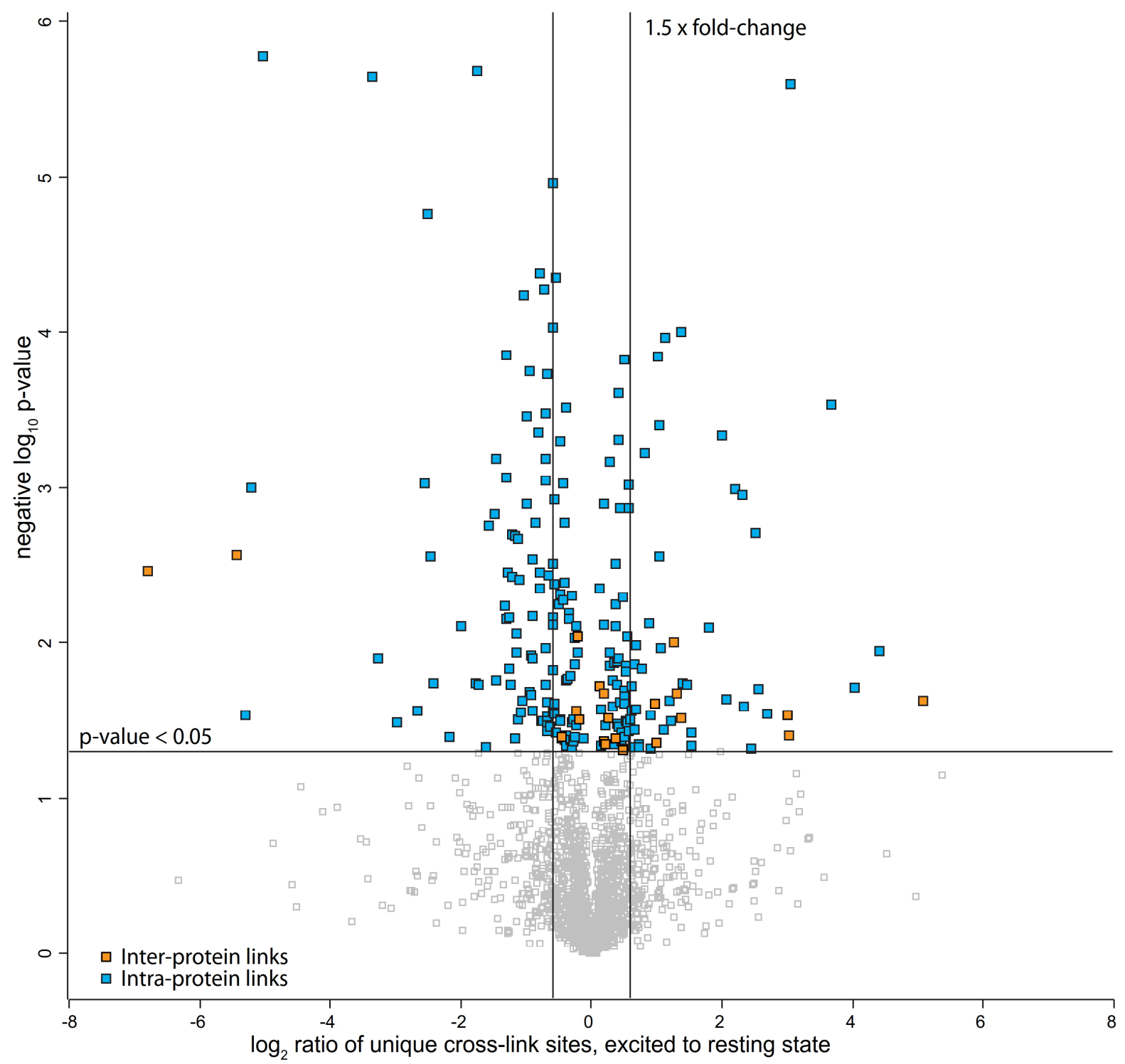

Figure 15: Scatter plot of significant fold-changes of unique cross-linked residues in synaptosomes under excited conditions in comparison to resting conditions plotted against the negative $\log _{10} p$-value. Synaptosomes were prepared from rat brains and cross-linked with isotopically labelled cross-linkers in an excited and resting state, respectively (chapter 2.2.4.4). Samples were pooled, and mitochondria were enriched (chapter 2.2.4.5). Cross-linked peptides were analyzed by LC-M Sand identified with pLink 1 at $1 \%$ FDR against a peptide database (chapter 2.4.3), followed by quantification with XiQ ${ }^{1}$ (chapter 2.4.4). Results from nine cross-linking replicates corresponding to three bioreplicates were merged, filtered for at least three valid values and a t-test was performed with a p-value cut-off below 0.05 (chapter 2.4.5).

Table 9: List of significantly changed unique cross-linked residues in synaptosomes upon stimulation $(1.3 \mathrm{mM} \mathrm{CaCl} 2,50 \mathrm{mM}$ $\mathrm{KCl}$ ) in comparison to resting conditions $(0.5 \mathrm{mM} \mathrm{EGTA}, 50 \mathrm{mM} \mathrm{KCl})$. Synaptosomes were prepared from rat brains and cross-linked with an isotopically labelled linker in an excited and resting state, respectively (chapter 2.2.4.4). Samples were pooled, and mitochondria were depleted (chapter 2.2.4.5). Cross-linked peptides were analysed by LC-M S and identified with plink 1 at $1 \%$ FDR with the peptide-focused approach (chapter 2.4.3), followed by quantification with XiQ ${ }^{1}$ (chapter 2.4.4). Results from nine cross-linking reaction replicates corresponding to three bioreplicates were merged, filtered for at least three valid values and at-test was performed with a p-value cut-off below 0.05 and a difference of at least 1.5 (chapter 2.4.5). A positive value in $\log _{2}$ ratio $\mathrm{Ca}^{2}+$ EGTA means an increased intensity of the respective cross-linked residue in the excited state and vice versa. Inter-protein links are highlighted in bold. Some links between protein isoforms occur as interlinks, but actually link to homologous peptides and are therefore probably intra-links. 


\begin{tabular}{|c|c|c|c|c|c|c|}
\hline Protein 1 & Protein 2 & AccessionID 1 - (residue) & AccessionlD 2 - (residue) & \# Replicates & $-\log _{10} p$-value & Log $_{2}$ ratio $\mathrm{Ca}^{2+} /$ EGTA \\
\hline $\begin{array}{l}\text { 4-nitrophenylphosphatase domain } \\
\text { and non-neuronal SNAP25-like } \\
\text { protein homolog } 1 \text { (C. elegans) }\end{array}$ & $\begin{array}{l}\text { 4-nitrophenylphosphatase domain } \\
\text { and non-neuronal SNAP25-like } \\
\text { protein homolog } 1 \text { (C. elegans) }\end{array}$ & G3V728_RAT - (73) & G3V728_RAT - (143) & 3 & 1.49 & -2.97 \\
\hline ADAM metallopeptidase domain 23 & ADAM metallopeptidase domain 23 & D3ZT36_RAT - (376) & D3ZT36_RAT - (376) & 9 & 3.40 & 1.05 \\
\hline ADP/ATP translocase 2 & ADP/ATP translocase 1 & ADT2_RAT - (245) & Q6P9Y4_RAT - (272) & 7 & 1.53 & 0.91 \\
\hline Alpha-centractin & Beta-centractin & ACTZ_RAT - (96) & B2RYJ7_RAT - (61) & 8 & 1.60 & 0.97 \\
\hline Amine oxidase [flavin-containing] B & Amine oxidase [flavin-containing] B & AOFB_RAT - (230) & AOFB_RAT - (222) & 9 & 3.85 & -1.31 \\
\hline \multirow[t]{2}{*}{ Annexin A6 } & Annexin A6 & Q6IMZ3_RAT - (75) & Q6IMZ3_RAT - (102) & 5 & 3.03 & -2.55 \\
\hline & & Q6IMZ3_RAT - (580) & Q6IMZ3_RAT - (610) & 4 & 1.95 & 4.40 \\
\hline $\begin{array}{l}\text { Aspartate aminotransferase, } \\
\text { mitochondrial }\end{array}$ & $\begin{array}{c}\text { Aspartate aminotransferase, } \\
\text { mitochondrial }\end{array}$ & AATM_RAT - (387) & AATM_RAT - (159) & 8 & 1.44 & 1.10 \\
\hline $\begin{array}{l}\text { ATP synthase subunit alpha, } \\
\text { mitochondrial }\end{array}$ & $\begin{array}{l}\text { ATP synthase subunit alpha, } \\
\text { mitochondrial }\end{array}$ & ATPA_RAT - (305) & ATPA_RAT - (167) & 3 & 1.33 & 0.65 \\
\hline $\begin{array}{l}\text { ATP-dependent 6- } \\
\text { phosphofructokinase }\end{array}$ & $\begin{array}{l}\text { ATP-dependent 6- } \\
\text { phosphofructokinase }\end{array}$ & PFKAP_RAT - (747) & PFKAP_RAT - (737) & 4 & 1.73 & -1.25 \\
\hline $\begin{array}{l}\text { Calcium/ calmodulin-dependent } \\
\text { protein kinase II, beta }\end{array}$ & $\begin{array}{l}\text { Calcium/calmodulin-dependent } \\
\text { protein kinase type Il subunit alpha }\end{array}$ & G3V9G3_RAT - (43) & KCC2A_RAT - (56) & 8 & 3.48 & -0.70 \\
\hline $\begin{array}{l}\text { Calcium/calmodulin-dependent } \\
\text { protein kinase II, beta }\end{array}$ & Neutral ceramidase & G3V9G3_RAT - (408) & ASAH2_RAT - (737) & 5 & 1.35 & 0.99 \\
\hline $\begin{array}{l}\text { Calcium/calmodulin-dependent } \\
\text { protein kinase type II subunit alpha }\end{array}$ & $\begin{array}{l}\text { Calcium/calmodulin-dependent } \\
\text { protein kinase type Il subunit alpha }\end{array}$ & KCC2A_RAT - (408) & KCC2A_RAT - (226) & 6 & 3.36 & -0.82 \\
\hline \multirow[t]{2}{*}{ Calcium-transporting ATPase } & Calcium-transporting ATPase & A0A0G2K9Q6_RAT - (238) & A0A0G2K9Q6_RAT - (525) & 6 & 1.50 & -1.13 \\
\hline & & A0A0G2K9Q6_RAT - (238) & A0A0G2K9Q6_RAT - (599) & 3 & 1.76 & -1.46 \\
\hline Calcium-transporting ATPase & $\begin{array}{l}\text { Plasma membrane calcium- } \\
\text { transporting ATPase } 1\end{array}$ & A0A0G2K9Q6_RAT - (525) & AT2B1_RAT - (193) & 9 & 5.60 & 3.06 \\
\hline \multirow[t]{2}{*}{ Calmodulin-3 } & Neuromodulin & CALM3_RAT - (22) & NEUM_RAT - (50) & 3 & 2.57 & -5.42 \\
\hline & & CALM3_RAT - (22) & NEUM_RAT - (56) & 5 & 2.46 & -6.78 \\
\hline Calreticulin & Calreticulin & CALR_RAT - (351) & CALR_RAT - (164) & 9 & 1.32 & 0.73 \\
\hline $\begin{array}{l}\text { CaM kinase-like vesicle-associated } \\
\text { protein }\end{array}$ & $\begin{array}{c}\text { CaM kinase-like vesicle-associated } \\
\text { protein }\end{array}$ & CAM KV_RAT - (260) & CAM KV_RAT - (294) & 5 & 1.38 & -1.17 \\
\hline $\begin{array}{l}\text { CDGSH iron-sulfur domain- } \\
\text { containing protein } 1\end{array}$ & $\begin{array}{l}\text { CDGSH iron-sulfur domain- } \\
\text { containing protein } 1\end{array}$ & CISD1_RAT - (104) & CISD1_RAT - (104) & 4 & 1.55 & -1.08 \\
\hline \multirow[t]{2}{*}{$\begin{array}{l}\text { Cell cycle exit and neuronal } \\
\text { differentiation protein } 1\end{array}$} & $\begin{array}{l}\text { Cell cycle exit and neuronal } \\
\text { differentiation protein } 1\end{array}$ & CEND_RAT - (25) & CEND_RAT - (38) & 4 & 1.93 & -1.14 \\
\hline & & CEND_RAT - (25) & CEND_RAT - (41) & 3 & 1.68 & -0.95 \\
\hline
\end{tabular}




\begin{tabular}{|c|c|c|c|c|c|c|}
\hline Protein 1 & Protein 2 & AccessionID 1 - (residue) & AccessionlD 2 - (residue) & \# Replicates & $-\log _{10} p$-value & Log $_{2}$ ratio $\mathrm{Ca}^{2+} /$ EGTA \\
\hline & & CEND_RAT - (25) & CEND_RAT - (42) & 7 & 2.45 & -1.29 \\
\hline & & CEND_RAT - (25) & CEND_RAT - (6) & 9 & 2.69 & -1.17 \\
\hline & & CEND_RAT - (42) & CEND_RAT - (33) & 9 & 1.43 & -0.69 \\
\hline & & CEND_RAT - (42) & CEND_RAT - (38) & 5 & 4.24 & -1.05 \\
\hline & & CEND_RAT - (48) & CEND_RAT - (41) & 6 & 2.67 & -1.13 \\
\hline & & CEND_RAT - (66) & CEND_RAT - (61) & 9 & 2.75 & -1.56 \\
\hline \multirow[t]{3}{*}{$\begin{array}{l}\text { Creatine kinase, mitochondrial } 1, \\
\text { ubiquitous }\end{array}$} & $\begin{array}{c}\text { Creatine kinase, mitochondrial } 1, \\
\text { ubiquitous }\end{array}$ & Q5BJT9_RAT - (140) & Q5BJT9_RAT - (417) & 5 & 2.43 & -1.22 \\
\hline & & Q5BJT9_RAT - (230) & Q5BJT9_RAT - (59) & 9 & 1.90 & -0.90 \\
\hline & & Q5BJT9_RAT - (344) & Q5BJT9_RAT - (417) & 7 & 1.83 & -1.27 \\
\hline Dynamin-1 & Dynamin-1 & DYN1_RAT - (87) & DYN1_RAT - (90) & 8 & 1.50 & 0.60 \\
\hline $\begin{array}{l}\text { Endoplasmic reticulum resident } \\
\text { protein } 29\end{array}$ & $\begin{array}{l}\text { Endoplasmic reticulum resident } \\
\text { protein } 29\end{array}$ & ERP29_RAT - (137) & ERP29_RAT - (69) & 5 & 1.73 & -0.70 \\
\hline Glutamate decarboxylase 2 & Glutamate decarboxylase 2 & DCE2_RAT - (431) & DCE2_RAT - (528) & 3 & 4.96 & -0.59 \\
\hline Heat shock cognate $71 \mathrm{kDa}$ protein & Heat shock protein 105 kDa & HSP7C_RAT - (56) & HS105_RAT - (272) & 4 & 1.51 & 1.37 \\
\hline Heat shock cognate $71 \mathrm{kDa}$ protein & Stress-70 protein, mitochondrial & HSP7C_RAT - (3) & GRP75_RAT - (206) & 4 & 1.53 & 3.01 \\
\hline Heat shock protein HSP 90-alpha & Heat shock protein HSP 90-alpha & HS90A_RAT - (74) & HS90A_RAT - (185) & 4 & 1.96 & 1.07 \\
\hline Hemoglobin subunit beta-1 & Hemoglobin subunit beta-1 & HBB1_RAT - (60) & HBB1_RAT - (67) & 6 & 1.87 & 0.66 \\
\hline Hexokinase-1 & Hexokinase-1 & HXK1_RAT - (777) & HXK1_RAT - (763) & 3 & 1.47 & -0.68 \\
\hline \multirow{10}{*}{$\begin{array}{l}\text { Inositol 1,4,5-trisphosphate receptor } \\
\text { type } 1\end{array}$} & $\begin{array}{l}\text { Inositol 1,4,5-trisphosphate receptor } \\
\text { type } 1\end{array}$ & F1LQX8_RAT - (168) & F1LQX8_RAT - (249) & 7 & 2.10 & -1.99 \\
\hline & & F1LQX8_RAT - (279) & F1LQX8_RAT - (436) & 9 & 2.18 & -0.90 \\
\hline & & F1LQX8_RAT - (335) & F1LQX8_RAT - (412) & 9 & 3.19 & -1.46 \\
\hline & & F1LQX8_RAT - (393) & F1LQX8_RAT - (249) & 9 & 2.44 & -0.67 \\
\hline & & F1LQX8_RAT - (397) & F1LQX8_RAT - (554) & 9 & 2.06 & -1.15 \\
\hline & & F1LQX8_RAT - (412) & F1LQX8_RAT - (1380) & 9 & 2.09 & 1.80 \\
\hline & & F1LQX8_RAT - (787) & F1LQX8_RAT - (1098) & 9 & 5.68 & -1.75 \\
\hline & & F1LQX8_RAT - (787) & F1LQX8_RAT - (1101) & 9 & 4.76 & -2.51 \\
\hline & & F1LQX8_RAT - (811) & F1LQX8_RAT - (787) & 8 & 3.06 & -1.31 \\
\hline & & F1LQX8_RAT - (1170) & F1LQX8_RAT - (1162) & 5 & 2.11 & -0.60 \\
\hline
\end{tabular}




\begin{tabular}{|c|c|c|c|c|c|c|}
\hline Protein 1 & Protein 2 & AccessionID 1 - (residue) & AccessionID 2 - (residue) & \# Replicates & $-\log _{10} p$-value & $\log _{2}$ ratio $\mathrm{Ca}^{2+} / \mathrm{EGTA}$ \\
\hline & & F1LQX8_RAT - (1380) & F1LQX8_RAT - (249) & 9 & 2.95 & 2.30 \\
\hline & & F1LQX8_RAT - (1380) & F1LQX8_RAT - (77) & 4 & 3.00 & -5.21 \\
\hline & & F1LQX8_RAT - (1869) & F1LQX8_RAT - (1870) & 6 & 1.63 & 1.20 \\
\hline $\begin{array}{l}\text { Inositol polyphosphate-5- } \\
\text { phosphatase A }\end{array}$ & $\begin{array}{c}\text { Inositol polyphosphate-5- } \\
\text { phosphatase A }\end{array}$ & D3ZZX1_RAT - (400) & D3ZZX1_RAT - (363) & 3 & 1.32 & 0.89 \\
\hline Integrin subunit alpha V & Integrin subunit alpha V & A0A0G2JVZ6_RAT - (165) & F1LZX9_RAT - (131) & 3 & 1.74 & 1.40 \\
\hline M etabotropic glutamate receptor 2 & M etabotropic glutamate receptor 2 & GRM 2_RAT - (43) & GRM 2_RAT - (94) & 8 & 2.16 & -1.26 \\
\hline \multirow[t]{2}{*}{ M yelin basic protein } & M yelin basic protein & MBP_RAT - (14) & M BP_RAT - (159) & 4 & 1.39 & -2.17 \\
\hline & & MBP_RAT - (14) & MBP_RAT - (166) & 3 & 1.55 & -0.60 \\
\hline $\begin{array}{c}\mathrm{Na}(+) / \mathrm{H}(+) \text { exchange regulatory } \\
\text { cofactor NHE-RF1 }\end{array}$ & $\begin{array}{c}\mathrm{Na}(+) / \mathrm{H}(+) \text { exchange regulatory } \\
\text { cofactor NHE-RF1 }\end{array}$ & NHRF1_RAT - (69) & NHRF1_RAT - (100) & 4 & 1.52 & -0.69 \\
\hline Neural cell adhesion molecule 1 & Neural cell adhesion molecule 1 & AOAOG2KOM8_RAT - (907) & A0A0G2KOM 8_RAT - (892) & 8 & 1.56 & -0.90 \\
\hline \multirow[t]{2}{*}{ NSF attachment protein gamma } & NSF attachment protein gamma & A0A0G2K350_RAT - (283) & A0A0G2K350_RAT - (281) & 8 & 1.56 & 0.62 \\
\hline & & A0A0G2K350_RAT - (291) & A0A0G2K350_RAT - (281) & 5 & 1.44 & 0.65 \\
\hline Phosphodiesterase & Phosphodiesterase & A0A0G2K876_RAT - (828) & A0A0G2K876_RAT - (804) & 3 & 1.73 & 1.46 \\
\hline \multirow[t]{12}{*}{$\begin{array}{l}\text { Plasma membrane calcium- } \\
\text { transporting ATPase } 1\end{array}$} & $\begin{array}{l}\text { Plasma membrane calcium- } \\
\text { transporting ATPase } 1\end{array}$ & AT2B1_RAT - (193) & AT2B1_RAT - (533) & 5 & 2.56 & 1.04 \\
\hline & & AT2B1_RAT - (237) & AT2B1_RAT - (601) & 6 & 2.56 & -2.46 \\
\hline & & AT2B1_RAT - (251) & AT2B1_RAT - (583) & 6 & 1.49 & -0.77 \\
\hline & & AT2B1_RAT - (252) & AT2B1_RAT - (583) & 9 & 3.46 & -1.00 \\
\hline & & AT2B1_RAT - (527) & AT2B1_RAT - (193) & 8 & 2.99 & 2.20 \\
\hline & & AT2B1_RAT - (544) & AT2B1_RAT - (193) & 8 & 3.34 & 2.00 \\
\hline & & AT2B1_RAT - (737) & AT2B1_RAT - (583) & 9 & 1.35 & 0.73 \\
\hline & & AT2B1_RAT - (778) & AT2B1_RAT - (349) & 3 & 1.83 & -0.60 \\
\hline & & AT2B1_RAT - (806) & AT2B1_RAT - (349) & 6 & 1.70 & 2.55 \\
\hline & & AT2B1_RAT - (807) & AT2B1_RAT - (351) & 4 & 1.42 & 1.53 \\
\hline & & AT2B1_RAT - (1070) & AT2B1_RAT - (921) & 9 & 1.62 & -0.67 \\
\hline & & AT2B1_RAT - (1078) & AT2B1_RAT - (807) & 6 & 1.56 & -2.67 \\
\hline \multirow[t]{2}{*}{$\begin{array}{l}\text { Plasma membrane calcium- } \\
\text { transporting ATPase } 2\end{array}$} & $\begin{array}{l}\text { Plasma membrane calcium- } \\
\text { transporting ATPase } 1\end{array}$ & AT2B2_RAT - (191) & AT2B1_RAT - (533) & 7 & 1.54 & 2.69 \\
\hline & & AT2B2_RAT - (235) & AT2B1_RAT - (583) & 9 & 1.84 & 0.77 \\
\hline
\end{tabular}




\begin{tabular}{|c|c|c|c|c|c|c|}
\hline Protein 1 & Protein 2 & AccessionlD 1 - (residue) & AccessionID 2 - (residue) & \# Replicates & $-\log _{10} p$-value & $\log _{2}$ ratio $\mathrm{Ca}^{2+} /$ EGTA \\
\hline & & AT2B2_RAT - (250) & AT2B1_RAT - (583) & 4 & 1.90 & -3.28 \\
\hline & & AT2B2_RAT - (254) & AT2B1_RAT - (583) & 9 & 5.65 & -3.35 \\
\hline & & AT2B2_RAT - (1101) & AT2B1_RAT - (807) & 9 & 2.54 & -0.90 \\
\hline $\begin{array}{l}\text { Plasma membrane calcium- } \\
\text { transporting ATPase } 2\end{array}$ & $\begin{array}{l}\text { Plasma membrane calcium- } \\
\text { transporting ATPase } 2\end{array}$ & AT2B2_RAT - (551) & AT2B2_RAT - (191) & 5 & 2.71 & 2.52 \\
\hline $\begin{array}{l}\text { Plasma membrane calcium- } \\
\text { transporting ATPase } 4\end{array}$ & $\begin{array}{l}\text { Plasma membrane calcium- } \\
\text { transporting ATPase } 4\end{array}$ & AT2B4_RAT - (337) & AT2B4_RAT - (735) & 5 & 1.53 & -5.29 \\
\hline $\begin{array}{l}\text { Plasma membrane calcium- } \\
\text { transporting ATPase } 4\end{array}$ & $\begin{array}{l}\text { Plasma membrane calcium- } \\
\text { transporting ATPase } 4\end{array}$ & AT2B4_RAT - (534) & AT2B4_RAT - (189) & 6 & 1.64 & 2.06 \\
\hline \multirow{6}{*}{$\begin{array}{l}\text { Platelet-activating factor } \\
\text { acetylhydrolase IB subunit alpha } \\
\text { Sarcoplasmic/endoplasmic } \\
\text { reticulum calcium ATPase } 2\end{array}$} & $\begin{array}{l}\text { Platelet-activating factor } \\
\text { acetylhydrolase IB subunit alpha }\end{array}$ & LIS1_RAT - (46) & LIS1_RAT - (54) & 5 & 1.50 & -0.69 \\
\hline & $\begin{array}{l}\text { Sarcoplasmic/ endoplasmic } \\
\text { reticulum calcium ATPase } 2\end{array}$ & AT2A2_RAT - (158) & AT2A2_RAT - (120) & 9 & 4.01 & 1.38 \\
\hline & & AT2A2_RAT - (205) & AT2A2_RAT - (492) & 6 & 1.74 & -2.42 \\
\hline & & AT2A2_RAT - (352) & AT2A2_RAT - (492) & 8 & 3.53 & 3.67 \\
\hline & & AT2A2_RAT - (492) & AT2A2_RAT - (205) & 9 & 5.78 & -5.03 \\
\hline & & AT2A2_RAT - (685) & AT2A2_RAT - (712) & 3 & 1.71 & 4.03 \\
\hline \multirow[t]{14}{*}{$\begin{array}{l}\text { Sodium/potassium-transporting } \\
\text { ATPase subunit alpha-1 }\end{array}$} & $\begin{array}{l}\text { Sodium/ potassium-transporting } \\
\text { ATPase subunit alpha-1 }\end{array}$ & AT1A1_RAT - (9) & AT1A1_RAT - (661) & 5 & 1.59 & 2.34 \\
\hline & & AT1A1_RAT - (67) & AT1A1_RAT - (434) & 5 & 1.32 & 2.44 \\
\hline & & AT1A1_RAT - (162) & AT1A1_RAT - (444) & 3 & 1.33 & -1.62 \\
\hline & & AT1A1_RAT - (212) & AT1A1_RAT - (487) & 9 & 3.84 & 1.02 \\
\hline & & AT1A1_RAT - (487) & AT1A1_RAT - (45) & 9 & 3.05 & -0.70 \\
\hline & & AT1A1_RAT - (487) & AT1A1_RAT - (212) & 9 & 3.97 & 1.13 \\
\hline & & AT1A1_RAT - (487) & AT1A1_RAT - (377) & 9 & 1.46 & -0.63 \\
\hline & & AT1A1_RAT - (487) & AT1A1_RAT - (625) & 8 & 1.50 & -0.75 \\
\hline & & AT1A1_RAT - (487) & AT1A1_RAT - (671) & 9 & 3.19 & -0.71 \\
\hline & & AT1A1_RAT - (671) & AT1A1_RAT - (212) & 8 & 2.83 & -1.48 \\
\hline & & ATIA1_RAT - (6 71) & AT1A1_RAT - (487) & 9 & 4.38 & -0.79 \\
\hline & & AT1A1_RAT - (726) & AT1A1_RAT - (21) & 9 & 3.75 & -0.94 \\
\hline & & AT1A1_RAT - (726) & AT1A1_RAT - (36) & 9 & 2.77 & -0.85 \\
\hline & & AT1A1_RAT - (726) & AT1A1_RAT - (37) & 9 & 2.41 & -1.11 \\
\hline
\end{tabular}




\begin{tabular}{|c|c|c|c|c|c|c|}
\hline Protein 1 & Protein 2 & AccessionID 1 - (residue) & AccessionID 2 - (residue) & \# Replicates & $-\log _{10} p$-value & Log $_{2}$ ratio $\mathrm{Ca}^{2+} / \mathrm{EGTA}$ \\
\hline & & AT1A1_RAT - (727) & AT1A1_RAT - (21) & 5 & 1.63 & -1.06 \\
\hline & & AT1A1_RAT - (727) & AT1A1_RAT - (36) & 9 & 2.15 & -1.31 \\
\hline & & AT1A1_RAT - (727) & AT1A1_RAT - (37) & 7 & 1.74 & -1.77 \\
\hline \multirow[t]{2}{*}{$\begin{array}{l}\text { Sodium/ potassium-transporting } \\
\text { ATPase subunit alpha-3 }\end{array}$} & $\begin{array}{l}\text { Sodium/ potassium-transporting } \\
\text { ATPase subunit alpha-1 }\end{array}$ & AT1A3_RAT - (202) & AT1A1_RAT - (212) & 4 & 1.40 & 3.03 \\
\hline & & AT1A3_RAT - (367) & AT1A1_RAT - (487) & 8 & 2.24 & -1.33 \\
\hline \multirow[t]{5}{*}{$\begin{array}{l}\text { Sodium/potassium-transporting } \\
\text { ATPase subunit alpha-3 }\end{array}$} & $\begin{array}{l}\text { Sodium/potassium-transporting } \\
\text { ATPase subunit alpha-3 }\end{array}$ & AT1A3_RAT - (349) & AT1A3_RAT - (146) & 5 & 2.12 & 0.87 \\
\hline & & AT1A3_RAT - (477) & AT1A3_RAT - (202) & 9 & 3.23 & 0.81 \\
\hline & & AT1A3_RAT - (661) & AT1A3_RAT - (202) & 9 & 2.35 & -0.78 \\
\hline & & AT1A3_RAT - (717) & AT1A3_RAT - (26) & 8 & 4.28 & -0.73 \\
\hline & & AT1A3_RAT - (717) & AT1A3_RAT - (27) & 9 & 3.73 & -0.69 \\
\hline Spectrin alpha chain & Ankyrin 2 & A0A0G2JZ69_RAT - (720) & $\begin{array}{c}\text { AOA0G2K6R9_RAT - } \\
\text { (1041) }\end{array}$ & 3 & 2.00 & 1.26 \\
\hline \multirow[t]{4}{*}{ Spectrin alpha chain } & Spectrin alpha chain & A0A0G2JZ69_RAT - (921) & A0A0G2JZ69_RAT - (943) & 9 & 1.72 & 0.61 \\
\hline & & A0A0G2JZ69_RAT - (1122) & A0A0G2JZ69_RAT - (1144) & 8 & 2.90 & -0.99 \\
\hline & & A0A0G2JZ69_RAT - (1205) & A0A0G2JZ69_RAT - (1105) & 3 & 1.73 & -1.74 \\
\hline & & A0A0G2JZ69_RAT - (1264) & A0A0G2JZ69_RAT - (1286) & 9 & 1.98 & 0.68 \\
\hline Spectrin alpha chain & Spectrin beta chain & A0A0G2JZ69_RAT - (921) & $\begin{array}{c}\text { AOAOG2K8W9_RAT - } \\
\text { (1527) }\end{array}$ & 3 & 1.63 & 5.07 \\
\hline Spectrin beta chain & Spectrin beta chain & A0A0G2K8W9_RAT - (394) & G3V6S0_RAT - (401) & 8 & 1.57 & 0.68 \\
\hline Succinate dehydrogenase & Succinate dehydrogenase & SDHA_RAT - (607) & SDHA_RAT - (608) & 3 & 1.49 & 1.22 \\
\hline Synaptotagmin I & Synaptotagmin II & SYT1_RAT - (332) & G3V6M3_RAT - (367) & 7 & 2.70 & -1.21 \\
\hline Synaptotagmin I & Synaptotagmin I & SYT1_RAT - (332) & SYT1_RAT - (366) & 9 & 2.46 & -0.80 \\
\hline Tripeptidyl-peptidase 2 & Tripeptidyl-peptidase 2 & TPP2_RAT - (187) & TPP2_RAT - (1204) & 5 & 1.66 & -0.92 \\
\hline Tubulin beta chain & Tubulin alpha-1A chain & G3V7C6_RAT - (439) & TBA1A_RAT - (96) & 3 & 1.67 & 1.31 \\
\hline Tubulin beta chain & Tubulin beta-2A chain & G3V7C6_RAT - (103) & TBB2A_RAT - (122) & 6 & 1.34 & 1.53 \\
\hline $\begin{array}{l}\text { Tyrosine-protein phosphatase non- } \\
\text { receptor type substrate } 1\end{array}$ & $\begin{array}{l}\text { Tyrosine-protein phosphatase non- } \\
\text { receptor type substrate } 1\end{array}$ & SHPS1_RAT - (467) & SHPS1_RAT - (445) & 4 & 1.97 & -0.71 \\
\hline $\begin{array}{c}\text { Voltage-dependent anion-selective } \\
\text { channel protein } 1\end{array}$ & $\begin{array}{c}\text { Voltage-dependent anion-selective } \\
\text { channel protein } 1\end{array}$ & VDAC1_RAT - (236) & VDAC1_RAT - (28) & 5 & 1.92 & -0.93 \\
\hline
\end{tabular}





\subsubsection{Significantly changed cross-links within ion-channels}

Quantitative XL-M S of synaptosomes was able to detect significant changes between the excited and resting state (table 9 ). Significant cross-linked residues especially accumulated in ion channels like SERCA, PM CA, Na $/ \mathrm{K}^{+}$ATPase and I3PR. Next, significantly changed cross-linked residues were mapped on reported model structures of these ion channels to deduce conformational changes in response to excitation.

Three of the four channels belong to the P-type ATPase superfamily, i.e. SERCA, PM CA, and $\mathrm{Na}^{+} / \mathrm{K}^{+}$ ATPase. Crystal structures were reported for all known conformations of SERCA including phosphorylated intermediates ${ }^{110-112}$, while model structures are not available for all states of PMCA and $\mathrm{Na}^{+} / \mathrm{K}^{+}$ATPase. Therefore, significantly changed cross-linked residues are analyzed on model structures of SERCA first, since major conformational changes are analogous for all three P-type ATPases.

All significantly quantified cross-links for SERCA were located within the head group, which also experiences the most dramatic conformational changes during a translocation cycle (figure 16). It was challenging to consolidate the data obtained in this study, which was based on two conditions, with a flexibility of at least five different conformations ${ }^{111,110,112}$.

Cross-linked residues that had a high abundance in an excited state (marked red in figure 16) should have a relatively short $\mathrm{C} \alpha-\mathrm{C} \alpha$ atom distance in a model structure in order that the structure is representative for the excited state. Accordingly, lower abundant cross-links in the excited state (marked blue in figure 16) should have long or overlength $\mathrm{C} \alpha$-C $\alpha$ atom distances in a model structure in order that the structure is representative for the excited state.

The same applies for the resting state: Cross-linked residues that had a high abundance in the resting state (marked blue in figure 16) should have a relatively short $\mathrm{C} \alpha$-C $\alpha$ atom distance in a model structure in order that the structure is representative for the resting state; Lower abundant cross-links in the resting state (marked red in figure 16) should have long or overlength $\mathrm{C} \alpha-\mathrm{C} \alpha$ atom distances in a model structure in order that the structure is representative for the resting state.

Based on this assumption, the E1 - $\mathrm{M} \mathrm{g}{ }^{2+}$ and E1 - P state and to some extent the E1 - $2 \mathrm{Ca}^{2+}$ state could be predominant conformations in an excited state. The low abundant cross-link in the excited state has a $\mathrm{C} \alpha$-C $\alpha$ atom distance beyond $30 \AA$ in these states (blue line between lysine 205 and 492, figure 16A-C). However, an excited state is characterized by high $\mathrm{Ca}^{2+}$ concentrations in the cytosol. SERCA in an $\mathrm{El}-\mathrm{M} \mathrm{g}^{2+}$ state, which has a high affinity for $\mathrm{Ca}^{2+}$ ions, should therefore quickly bind $\mathrm{Ca}^{2+}$ and proceed to the following states. In addition, the transition from E1 to E2 state involves a $110^{\circ}$ rotation of the $A$ domain and a reorganization of transmembrane helices for an opening towards the lumen ${ }^{111}{ }^{112}$, which is probably the slowest step in the cycle. Therefore, the significantly changed cross-links in an excited state match best to SERCA in an E1 - $2 \mathrm{Ca}^{2+}$ and E1 - P state. Accordingly, the predominant state for the resting state is probably the E2 state, because the high abundant cross-link in the resting state between lysine 205 and 492 (blue line, 14.7 $\AA$, figure 16D) has the shortest $\mathrm{C} \alpha$-C $\alpha$ atom distance in the E2 state. 


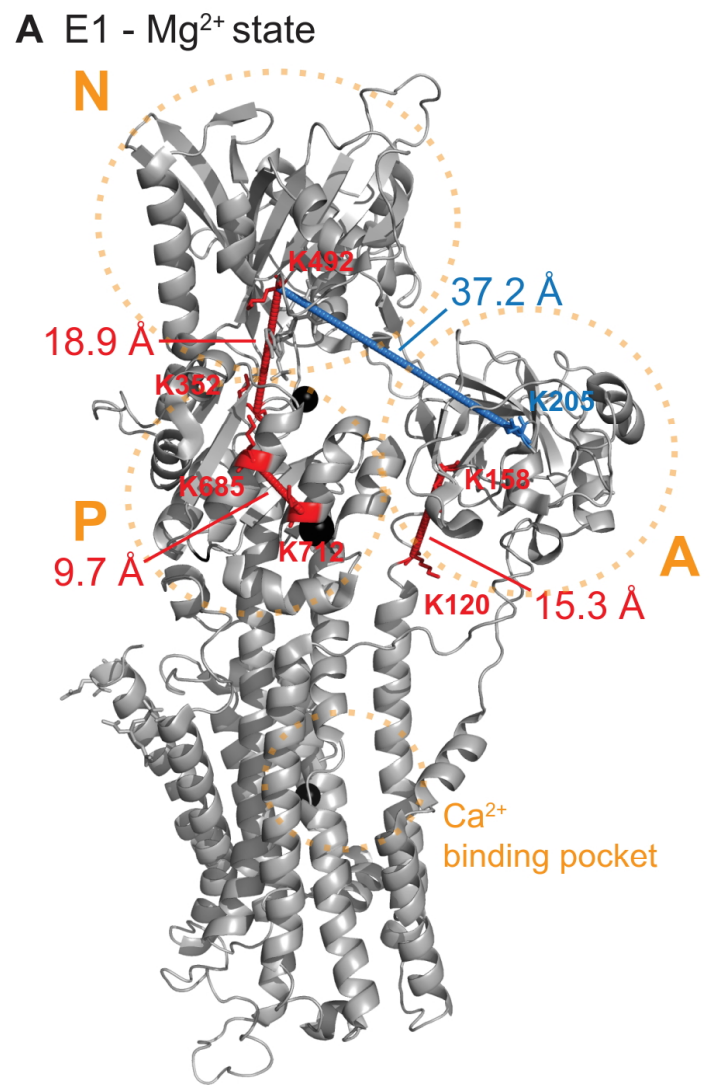

\author{
B E1 - $2 \mathrm{Ca}^{2+}$ state
}
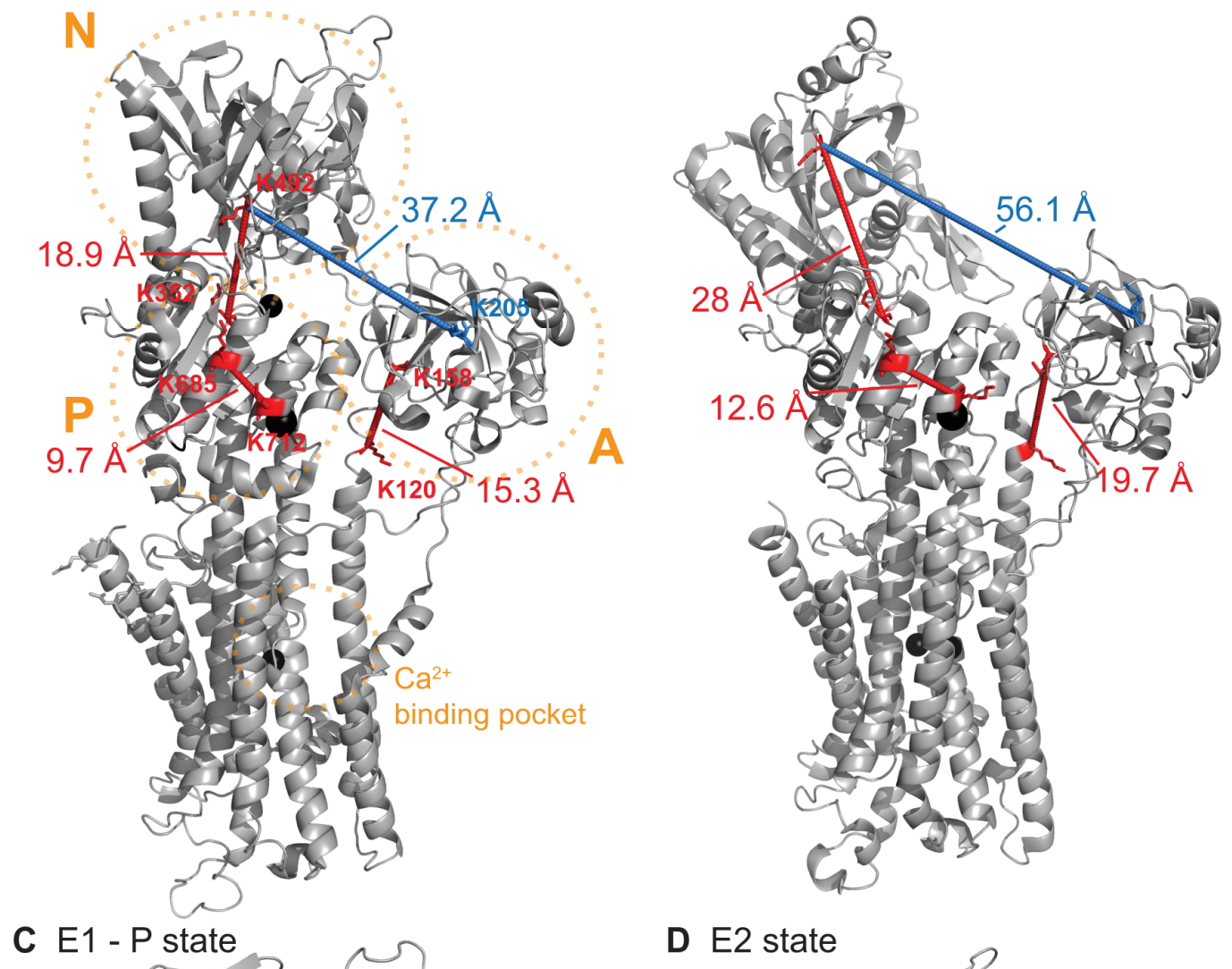

D E2 state

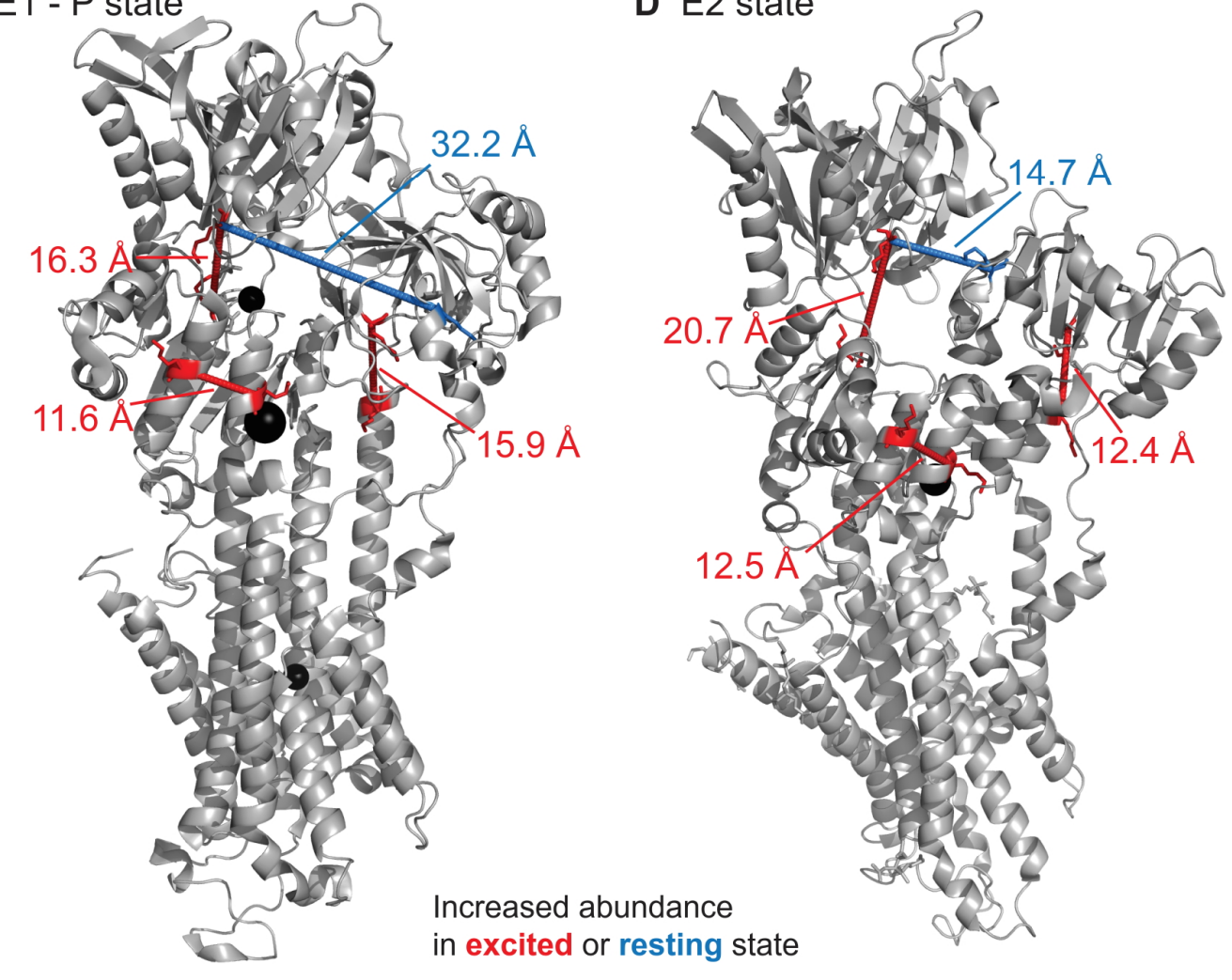

Figure 16: Significantly changed cross-links displayed on different conformations of the sarcoendoplasmic Ca2+ transporting ATPase. Cross-links from table 9 were plotted with pyM OL on highly homologous crystal structures of the E1 - M g2+(A, pdb: 3w5a) ${ }^{112}, \mathrm{E} 1-2 \mathrm{Ca}^{2+}(\mathbf{B}, \mathrm{pdb}: 1 \mathrm{su} 4)^{110}, \mathrm{E} 1-\mathrm{P}(\mathbf{C}, \mathrm{pdb}: 3 \mathrm{ba6})^{111}$, and E2 state (D, pdb: 3w5c) ${ }^{112}$ from rabbit. A (Actuator), P (phosphorylation), and $\mathrm{N}$ (nucleotide binding) domains of the head group and the $\mathrm{Ca}^{2}+$ binding pocket in the transmembrane domain are indicated by orange dashed circles in A. Cross-links and $\mathrm{C} \alpha-\mathrm{C} \alpha$ distances that had increased and decreased intensities in an excited state are depicted as red and blue lines, respectively. Cross-linked lysine positions are shown in $\mathbf{A}$. Bound ions are shown as black spheres. 
Considerably more significant cross-links were identified for the structurally similar PM CA, for which only a structure of the E1 - $\mathrm{M} \mathrm{g}^{2+}$ state was reported (figure 17) ${ }^{146}$. Cross-links mostly clustered in the head group between the actuator $(\mathrm{A})$ and nucleotide-binding $(\mathrm{N})$ domain. In addition, some cross-links were significantly altered from the phosphorylation $(\mathrm{P})$ domain to an unresolved loop and the Cterminus, which are both flexible. Similar to the cross-links observed for SERCA (figure 16), most crosslinks from $\mathrm{A}$ to $\mathrm{N}$ domain are less abundant in an excited state, while one cross-link from the $\mathrm{P}$ to $\mathrm{N}$ domain was more abundant. These cross-links match an E1 - $2 \mathrm{Ca}^{2+}$ and E1 - P state with the N domain swung open. In addition three cross-links on the right side of the $A$ and $N$ domain (figure 17B, red crosslinks, K193-K544, K193-K533 with 21 - 28 A distance) were more abundant, which was in agreement with the E1 state and a not yet rotated A domain. Interestingly, a flexible and unresolved loop (figure 17A, black dashed line) and the C-terminus, to which several significantly changed cross-links were identified, are regulatory elements. The C-terminus is known to have an auto-inhibitory function, while the unresolved loop contains an activating phospholipid-binding domain ${ }^{146,147}$. During excitation, there is a high calcium concentration in the cytoplasm and PMCA should supposedly be highly active to decrease the calcium concentration. The significantly changed cross-links to regulatory domains are therefore implications for a conformational change in the PM CA structure towards a probably more active and uninhibited state. 
A

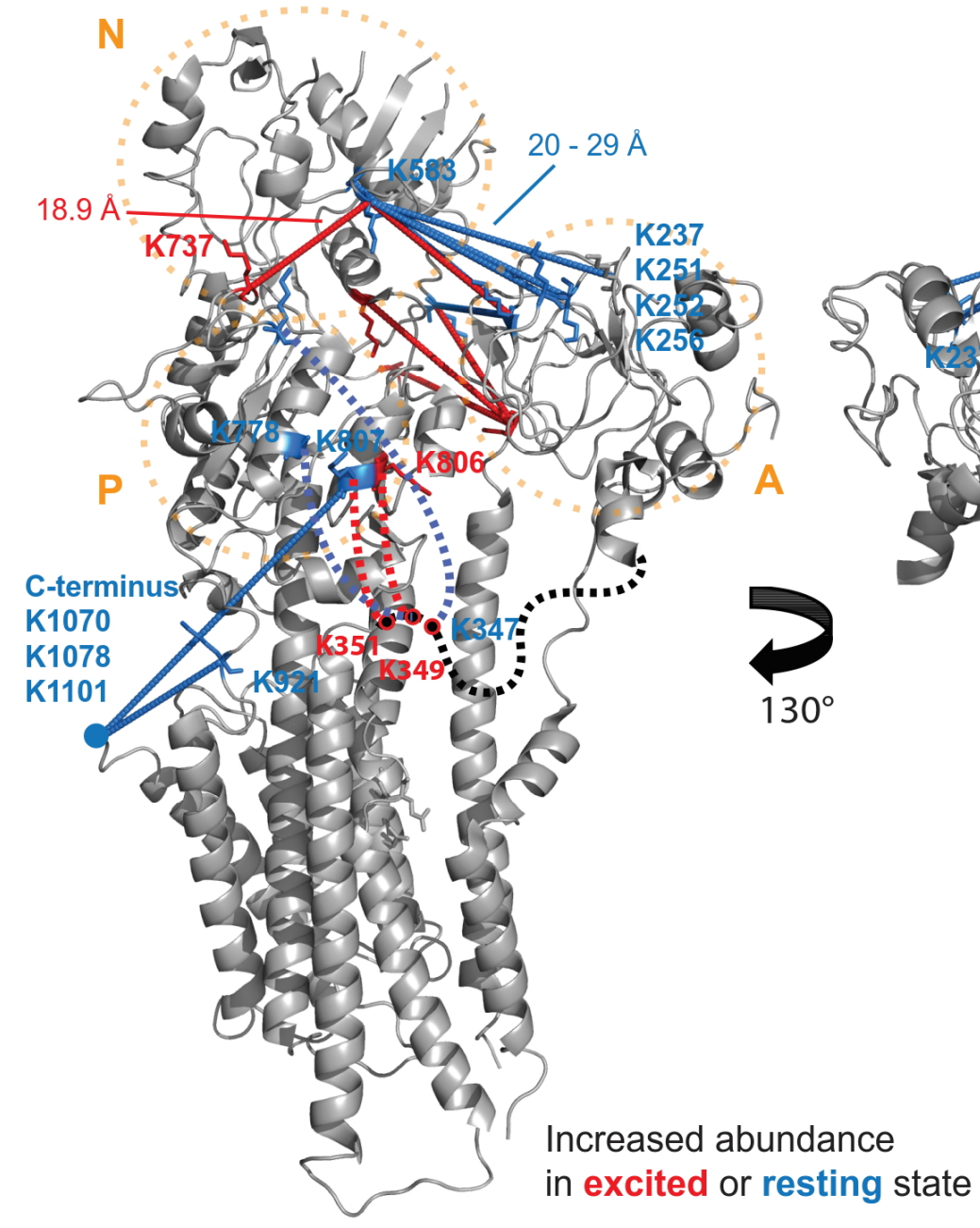

B

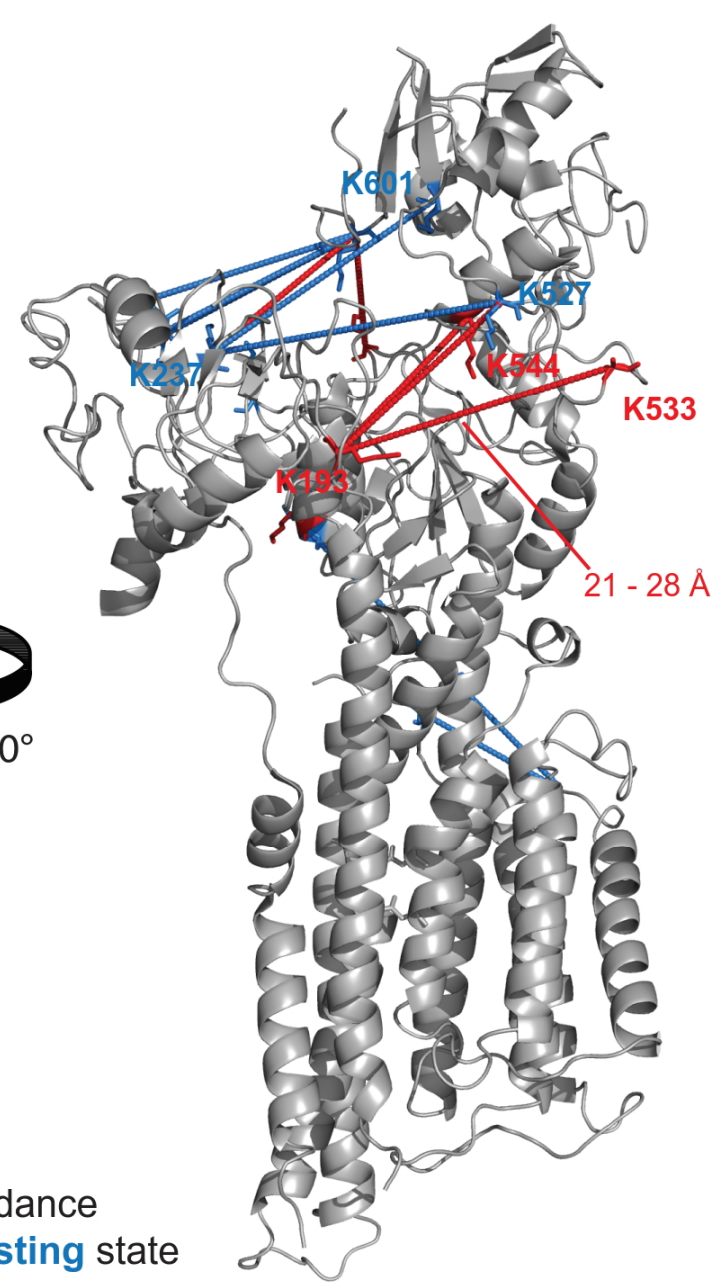

Figure 17: Significantly changed cross-links within plasma membrane Ca2+transporting ATPase (PMCA). Cross-links from table 9 were plotted with pyM OL on a highly homologous cryo-EM structure of human PM CA presumably in an E1 - M g ${ }^{2+}$ state (pdb: 6a69)146. A (Actuator), P (phosphorylation), and N (nucleotide binding) domains of the head group are indicated by orange dashed circles. The orientation in $\mathbf{A}$ is similar to figure 16 , while $\mathbf{B}$ is rotated by $130^{\circ}$. Cross-links and $\mathrm{C} \alpha-\mathrm{C} \alpha$ distances that had increased and decreased intensities in an excited state are depicted as red and blue lines, respectively. Cross-linked lysine positions are indicated. Flexible loops that were not resolved in the crystal structure were drawn schematically. Crosslinks to the unresolved C-terminus are drawn to the last amino acid shown in the structure, instead, which is 17 and 38 amino acids off, respectively. 
A different distribution of significantly changed cross-links was observed for $\mathrm{Na}^{+} / \mathrm{K}^{+} \mathrm{ATPase}$, for which model structures in the E1-P $3 \mathrm{Na}^{+}$and E2-P $2 \mathrm{~K}^{+}$state are available(Figure 18)115, 148. Under excited conditions the gradient of sodium and potassium ions across the membrane is disturbed. The ion antiporter is constantly shuttling ions, because it almost instantly binds potassium ions on the extracellular and sodium ions on the cytoplasmic side after completing a translocation cycle. Accordingly, the cross-linking pattern in an excited state was neither matching the E1 nor the E2 state unanimously.

As a reminder, cross-linked residues that had a high abundance in an excited state (marked red) should have a relatively short $\mathrm{C} \alpha-\mathrm{C} \alpha$ atom distance in a model structure in order that the structure is representative for the excited state. Accordingly, lower abundant cross-links in the excited state (marked blue) should have long or overlength $\mathrm{C} \alpha$-C $\alpha$ atom distances in a model structure in order that the structure is representative for the excited state.

On the one hand, there were two cross-links between the $A$ and $N$ domain that supported the $\mathrm{E} 1$ state similar to PM CA and SERCA. One cross-link was less abundant (figure $18, K 45-K 487$, blue line, $36 \AA$ in $A, 30 \AA$ in $B$ ) and one cross-link was more abundant (figure 18, K67-K434, red line, $19 \AA$ in $A, 45 \AA$ in $B$ ) on the right side of the $A$ and $N$ domain. In addition, three cross-links to the $\mathrm{N}$-terminus that were less abundant upon stimulation were longer in E1 state than in E2 state (figure 18, K726/K727 to $\mathrm{K} 21 / \mathrm{K} 36 / \mathrm{K} 37,27-28 \AA$ in $\mathrm{A}, 13-16 \AA$ in $\mathrm{B}$ ), which supports the $\mathrm{E} 1$ state being representative for the excited state.

On the other hand, several cross-links supported the E2 state to be predominant upon stimulation. four cross-links from the $P$ to the $N$ and $A$ domain that were less abundant in the excited state were longer in E2 state (figure 18, K625/K671/K377 to K487, 18-24 $\AA$ in A, 27-35 $\AA$ in B).

A high abundant cross-link between the $N$ and $A$ domain (figure $18 A, K 212-K 487,17.6 \AA$ in $A, 18.9 \AA$ in $B$; the link disappears behind another link) was detected in the excited state that had a similar $\mathrm{C} \alpha$ - $\mathrm{C} \alpha$ atom distance in the E1 and E2 state. Interestingly, this cross-link was almost within the cross-link distance of DSS when mapped as an inter-protein cross-link (figure 18C). Assuming that the $\mathrm{Na}^{+} / \mathrm{K}^{+}$ ATPase alpha subunit dimerizes ${ }^{149}$ in the same fashion as visible in the crystal lattice in E1-P state, the $\mathrm{C} \alpha$ - $\mathrm{C} \alpha$ atom distance of these cross-links are only slightly longer than $30 \AA$ (figure 18C, K202-K487 and K212-K477, red lines of 31.6 and $32.7 \AA$ length; respective intra-links are depicted in salmon-red). In addition, one cross-link was identified to the same amino acid residue in two different isoforms, which necessarily was an inter-protein cross-link (figure 18C, K202-K212, red line of $21.7 \AA$ length). Another cross-link to the $\mathrm{N}$-terminus with a higher abundance under excited conditions was shorter, when plotted as inter-protein cross-link (figure 18C, K651-K11, K661-K21, red lines of 34.7 and $38.2 \AA$ length). These three cross-links suggest that excited conditions favour the dimerization of the alpha subunit of $\mathrm{Na}^{+} / \mathrm{K}^{+}$ATPase. A dimerization of the alpha subunit was described in the past ${ }^{149}$. 

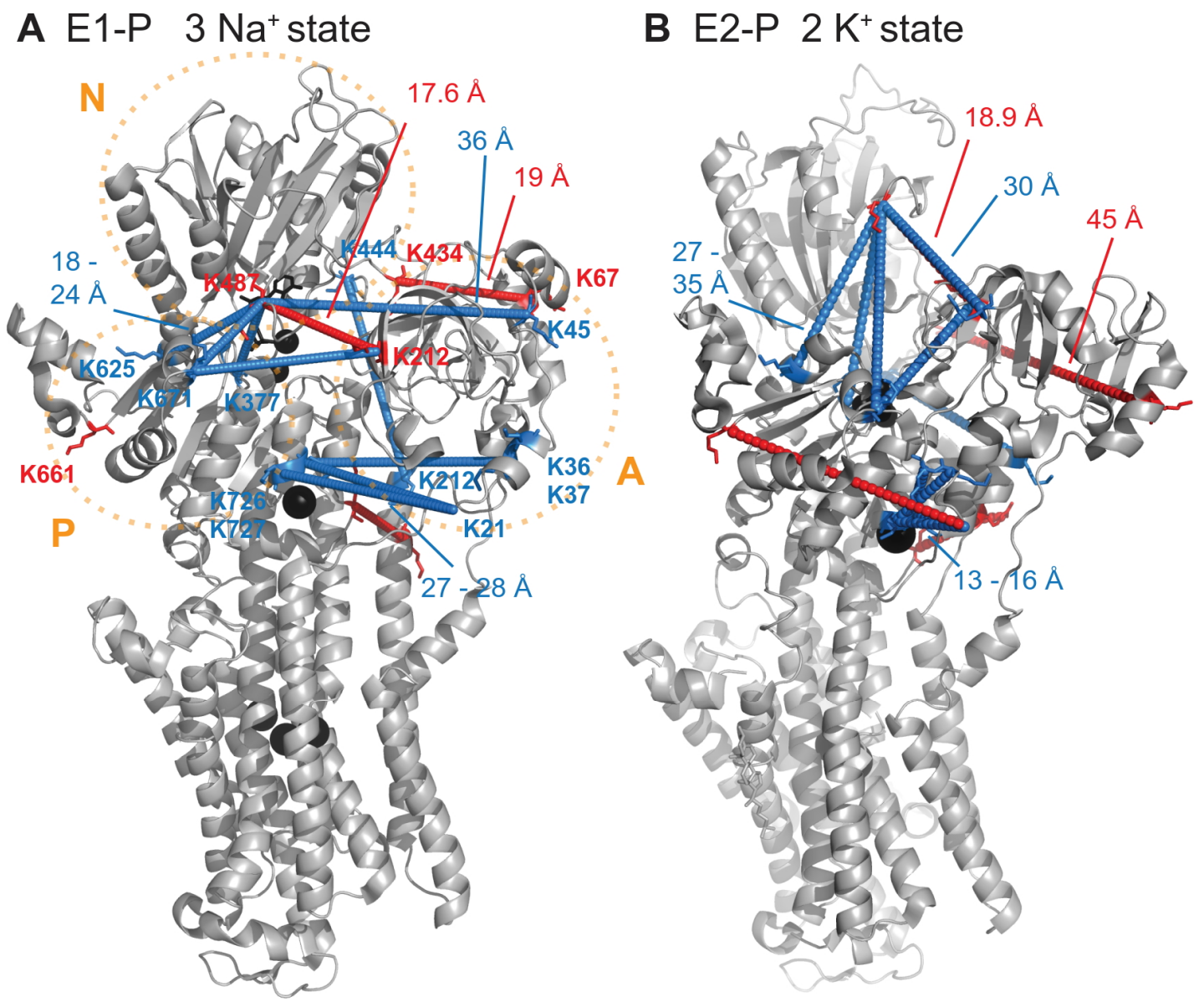

C top view of E1-P state as dimer Isoform 3

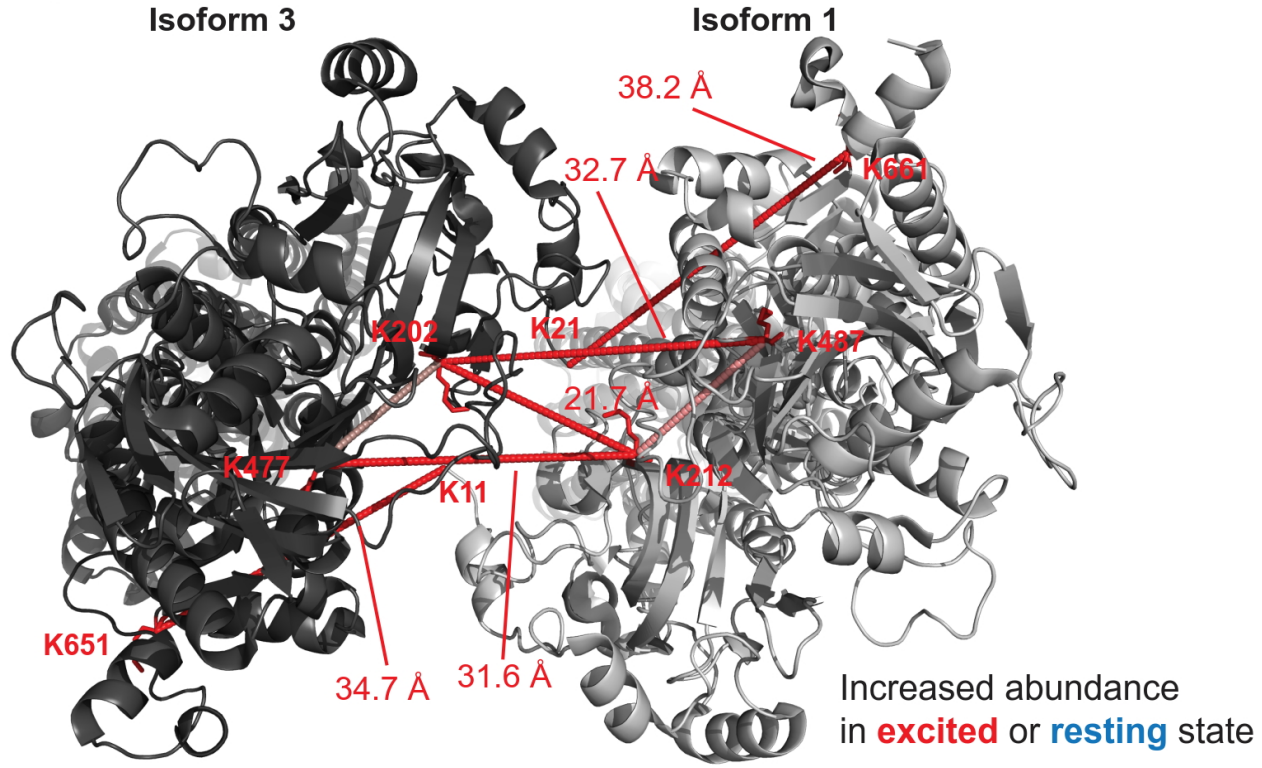

Figure 18: Significantly changed cross-links for $\mathrm{Na}^{+} / \mathrm{K}^{+} \mathrm{ATP}$ ase. Cross-links from table 9 were plotted with pyM OL on a highly homologous crystal structure of pig Na+/K+ATPase alpha subunit in a Na+-bound state preceding E1-P (A, pdb: $3 w g v)^{115}$ and a $\mathrm{Na}^{+} / \mathrm{K}^{+} \mathrm{ATPase}$ alpha subunit in E2-P - $2 \mathrm{~K}^{+}$state (B, pdb: 2zxe) ${ }^{148}$ from spiny dogfish. A (Actuator), $\mathrm{P}$ (phosphorylation), and $\mathrm{N}$ (nucleotide binding) domains of the head group are indicated by orange dashed circles. The orientation in $\mathbf{A}$ and $\mathbf{B}$ is similar to figure 16. Cross-linked lysine positions are indicated. Cross-links and $\mathrm{C} \alpha$ - $\mathrm{C} \alpha$ atom distances that had increased and decreased intensities in an excited state are depicted as red and blue lines, respectively. C: Suggested and actually identified inter-links were exemplary plotted onto the E1-P structure115 from A, which was present as homodimer in the crystal lattice. The respective intra-links are displayed in salmon-red. Bound ions and ligands are shown as black spheres and sticks. 
Apart from P-type ATPases, other calcium channels displayed significantly changed cross-links, as well, for example inositol-1,4,5-trisphosphate receptor (I3PR). I3PR is a homotetrameric calcium channel residing in the membrane of intracellular calcium stores like the endoplasmic reticulum ${ }^{150}$. Upon binding its ligand inositol-1,4,5-trisphosphate as a result of cellular signaling, it is undergoing a conformation change in the C-terminal alpha-helix, which together with the other three subunits constitutes the ion pore. Subsequently, several hydrophobic amino acid residues are not protruding into the ion channel anymore leading to an ion-conducting state (figure 19, view on top of I3PR, four central alpha-helices with a closed pore on the left and open pore on the right) ${ }^{114}$. The biological condition in the present study was not a trigger leading to an immediate effect on I3PR via its ligand. Nonetheless, several significantly changed cross-links were identified (figure 19). Most of the crosslinks that were significantly less abundant in an excited state were close to the ligand binding pocket indicating a conformational change upon ligand binding. Surprisingly, two cross-links were quantified with a higher abundance under excited conditions, which by far exceeded the distance constraints of the cross-linker. These cross-links were not explained by both available structures of I3PR in a closed and open state thereby implying a possible third conformation for I3PR that includes a major domain movement towards the ligand binding pocket.

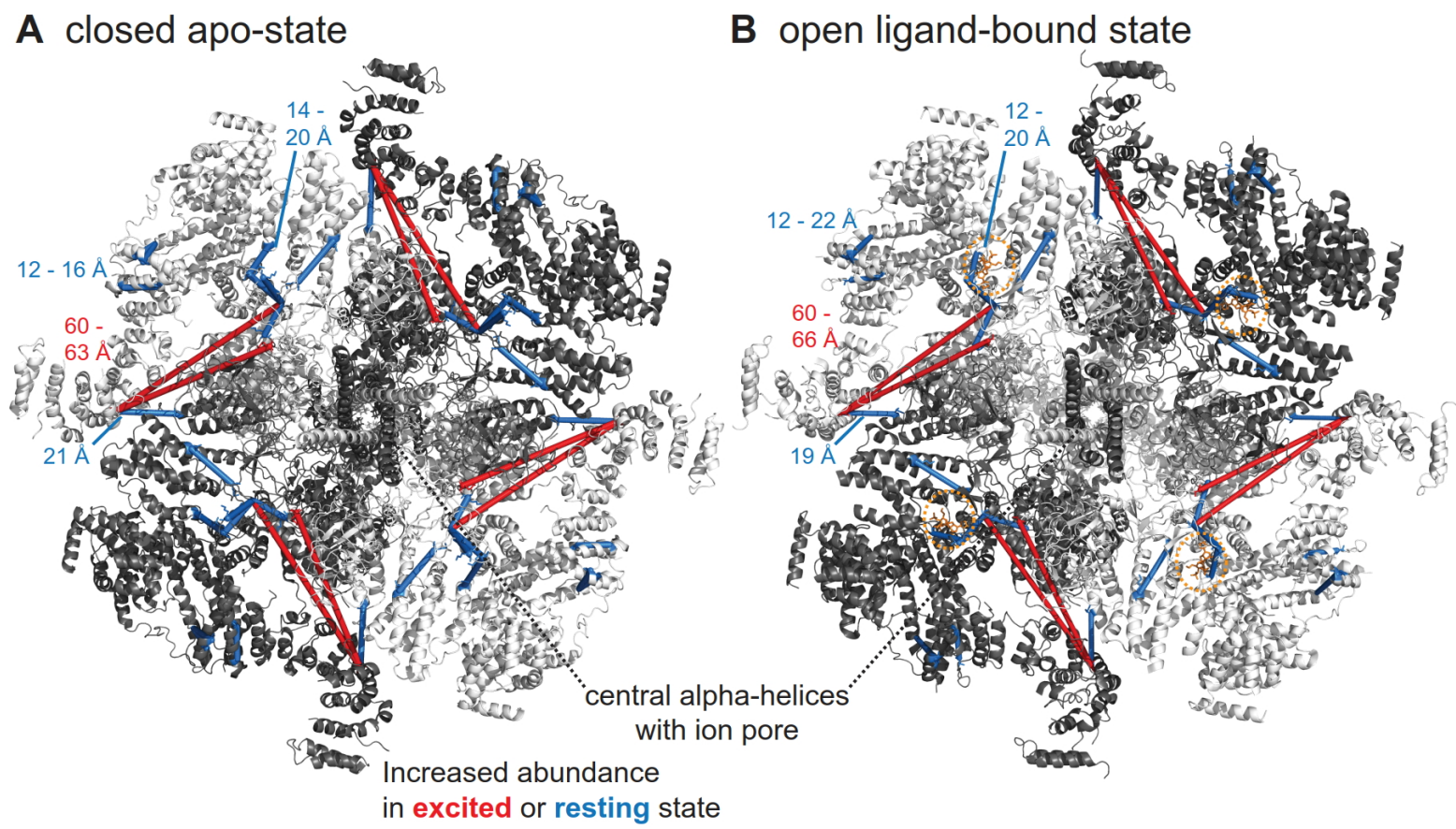

Figure 19: Significantly changed cross-links within homotetrameric inositol-1,4,5-trisphosphate receptor (I3PR). Cross-links from table 9 were plotted with pyMOL on a cryo-EM structure of rat I3PR in a closed apo-state (A, pdb: 6mu2, left) ${ }^{114}$ and open ligand-bound state (B, pdb: 6mu1, right) ${ }^{114}$. Cross-links and $\mathrm{C} \alpha-\mathrm{C} \alpha$ distances that had increased and decreased intensities in an excited state are depicted as red and blue lines, respectively. The bound ligand (orange) is encircled by orange dashes. 


\subsubsection{Significantly changed cross-links within calcium-binding proteins}

Several significantly changed cross-links were observed in proteins that are binding calcium either directly or indirectly via binding to calcium/calmodulin complex. Calmodulin itself exhibited two interlinks to neuromodulin that were 50 to 100 times less abundant during stimulation in comparison to resting state (table 9). Neuromodulin is a protein with a high concentration in neuronal growth cones and is associated with axon elongation and synaptic plasticity ${ }^{100}$. Neuromodulin binds to calmodulin with a higher affinity in the absence of calcium and with a lower affinity in presence of calcium and potassium $^{100}$. This property of neuromodulin would explain the observed significantly lower abundant inter-protein cross-links between neuromodulin and calmodulin under excited conditions. Although calmodulin is known to interact with a wide variety of effector proteins in a calcium-dependent manner, no other significantly changed inter-links were detected involving calmodulin. Nonetheless, several cross-links were quantified in calmodulin effector proteins like calcium/calmodulin-dependent protein kinase II (CamkII), spectrins, and Camkv.

Various inter- and intra-links were identified between and within the four Camkll chains A, B, G, and D (figure 12), which are known to oligomerize to homo- and heterododecamers with their hub domains, while the kinase domains are flexibly moving around the central hub-complex (figure 20) ${ }^{102}$. Apart from two significantly higher abundant cross-links under excited conditions with a ratio below 1.5 (supplementary table 4), two significantly less abundant cross-links were identified (figure 20, right). One cross-link was spanning from the kinase to the hub domain, which is overlength in case of an extended kinase domain but would be within the cross-linker-distance in a compact formation of the monomer due to a flexible unstructured cross-linker. Such a compact structure of Camkll is associated with an auto-inhibited state, because the regulatory alpha-helix is buried between the kinase and hub domain ${ }^{151}$. The other cross-link involves K43, which enables ATP-binding prior to substrate phosphorylation in the catalytic core of the protein ${ }^{152}$. It might be explained by the sterical obstruction by a bound ATP molecule or conformational changes that are accompanied by ATP binding. The decrease of both cross-links under excited conditions are therefore in accordance with a catalytically active Camkll complex upon stimulation. Camk2 also exhibited a significantly more intense inter-link to neutral ceramidase in a stimulated state. Although only one cross-link was significantly changed, in total three unique cross-links were identified to two different residues K344 and 371 within Camk2A and to a homologous peptide within Camk2B at position K408. 


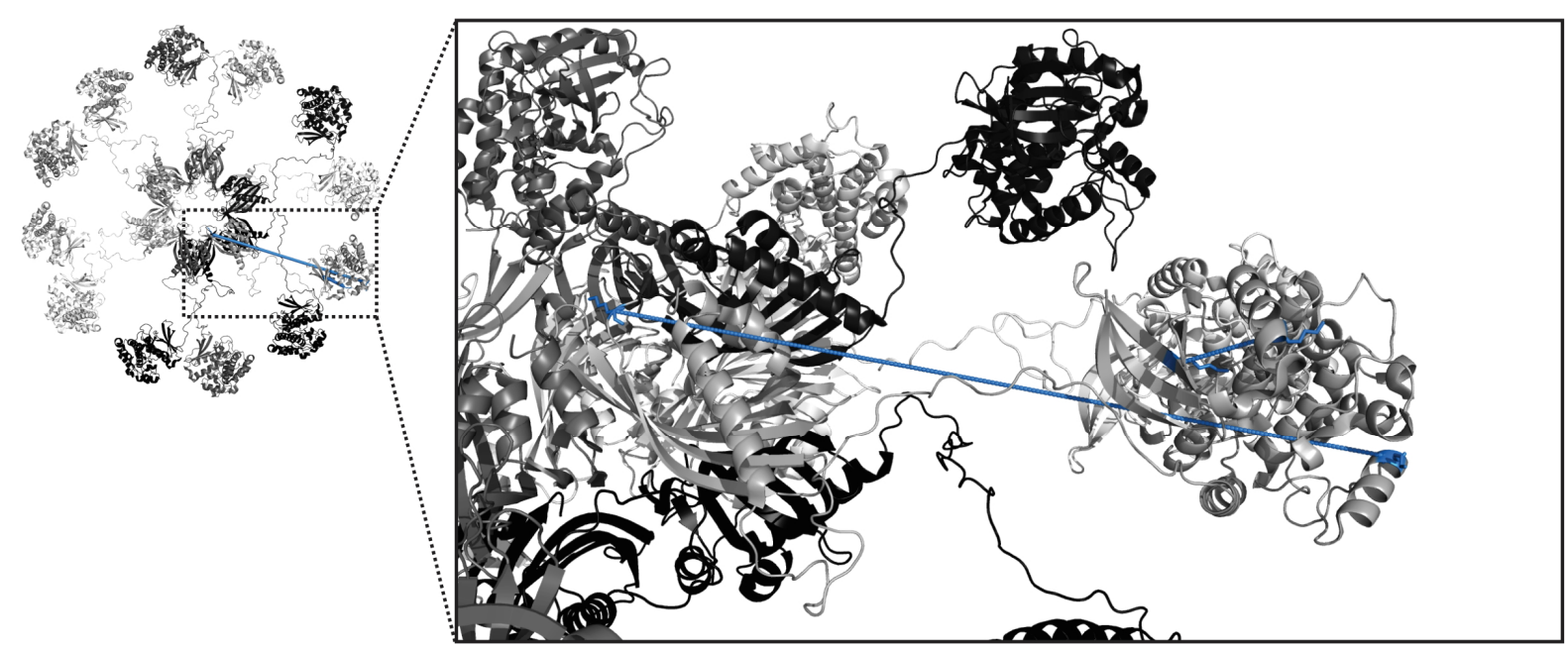

Figure 20: Significantly changed cross-links within calcium/calmodulin-dependent protein kinase II (CamkII). Cross-links from table 9 were plotted with pyM OL on a cryo-EM structure of rat CamkIIA (pdb: 5u6y) ${ }^{102}$. Cross-link distances that had decreased intensities in an excited state are depicted as blue lines.

Another calmodulin effector protein exhibiting a significantly changed cross-link was Camkv, which is a vesicle- and membrane-associated protein. Although, it is binding calmodulin in presence of calcium, there was no kinase activity detectable in vitro ${ }^{153}$ and mutation of this pseudo-kinase domain had no effect on its regulatory effects in dendritic spine morphogenesis ${ }^{154}$. However, the less abundant crosslink under stimulatory conditions between residues K260 and K294 was located within the ATPase domain (residues 24 to 286), which is N-terminal of the calmodulin binding domain. The decreasing intensity of this cross-link therefore indicates a calcium-induced structural change that involves the catalytically inactive ATPase domain of Camkv.

Many siginificantly changing inter- and intra-links were observed for spectrin alpha chain and its interactors spectrin beta chain, as well as ankyrin-2 (figure 21). All significantly changed cross-links clustered between residue 700 and 1,300 of the alpha chain of spectrin. Spectrin alpha is known to bind both calmodulin and calcium. The EF-hand calcium binding domains are at the C-terminus of spectrin alpha, though, while the calmodulin binding domain is closer to the changing cross-links between residue 1,187 and $1,210^{155,156}$. The proximity to the calmodulin binding site indicates that the changing cross-links might rather be an effect of calcium/calmodulin binding than binding of calcium alone. Under excited conditions, one cross-link from spectrin alpha to beta chain and to ankyrin-2 increased in its abundance implying that interactions were affected upon stimulation. Then again, only one cross-link between the two spectrin chains exhibited a significant change, while many more were insignificant. Inter-protein cross-links between spectrin alpha and beta span from the $\mathrm{N}$-terminus of one subunit to the $\mathrm{C}$-terminus of the other and vice versa (figure 21 , grey dashed lines). This confirms an antiparallel binding of the two chains that was described before ${ }^{157}$. 


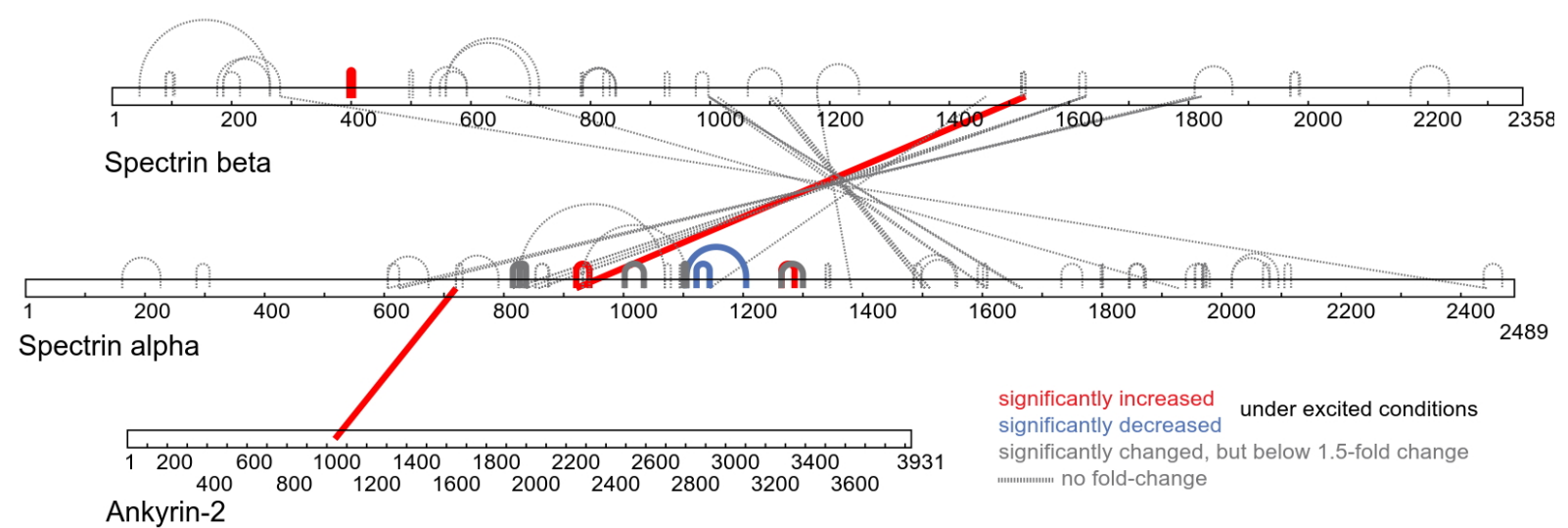

Figure 21: Significantly changed cross-links within and between spectrin alpha, beta and ankyrin-2. Significant cross-links from table 9 (red for more and blue for less abundant in an excited state), cross-links with a fold-change below 1.5 (grey) and insignificant or merely identified cross-links (dashed, electronic supplementals) were visualized with xiNET.

The main calcium sensor in SNARE mediated exocytosis Syt1 revealed two significantly decreased cross-links under stimulatory conditions (figure 22). The cross-link between K332 and K366 was identified to the loop on top of the C2B domain responsible for calcium binding ${ }^{158}$ and was possibly decreasing under stimulation due to sterical hindrance from calcium ions binding to the domain (figure 22 , left). Another cross-link between nearby lysine residues in the C2A domain had a slightly reduced intensity ratio of 0.72 in the excited state in comparison to resting state (supplementary table 4, figure 22 , right).

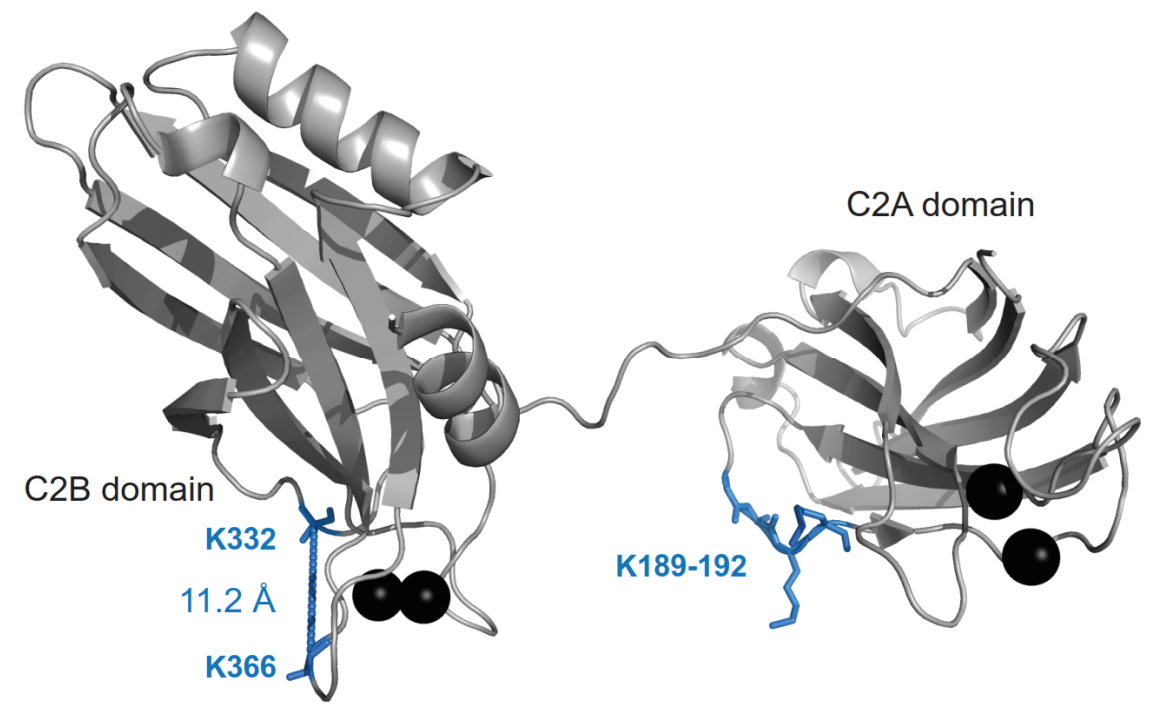

Figure 22: Significantly changed cross-links within synaptotagmin I (Syt1). Cross-links from table 9 were plotted with pyM OL on a crystal structure of rat Synaptotagmin I (pdb: $5 \mathrm{ccg})^{158}$. The cross-link between lysine 332 and 366 had a decreased intensity in an excited state and is depicted as blue line. One cross-link with a significant fold-change below 1.5 was identified between neighboring lysines residues (K189-192). Bound calcium ions are depicted as black spheres. 
Similar to Syt1, annexins are able to bind to phospholipids in a calcium-dependent manner ${ }^{159}$. Anxa6 consists of two perpendicular lobes $A$ and $B$, each containing four annexin repeats, each of which is potentially able to bind two calcium ions ${ }^{159}$. In presence of high calcium concentrations, loops on top of annexin repeats are binding ions, which promote the binding to phospholipid head groups. M embrane binding is encompassed by a conformational change of the lobes from a perpendicular to a co-planar orientation to each other. Once bound to membranes, a calcium channel activity was observed for Anxa6 $6^{160}$, which was also reported for other proteins of the annexin family ${ }^{161}$. The largest conformational change upon calcium influx under excited conditions was expected to be the switch from perpendicular to co-planar orientation of the two lobes. Such a movement was not identified by quantitative cross-linking in this thesis. Instead, two significantly changed cross-links between annexin repeat domains were observed (figure 23). Similar to Syt1, the cross-link with a lower abundance under excited conditions between K75 and K102 could be explained with a sterical obstruction by the neighbouring calcium ion (figure 23B). Interestingly, the two cross-links are virtually identical, spanning between the central left to the lower annexin repeat domain within the context and orientation of the lobes in figure 23. The cross-links imply that apart from the relative movement of the two lobes, the annexin repeat domains might also move relative to each other in response to calcium influx and this movement is contrary for the two lobes.

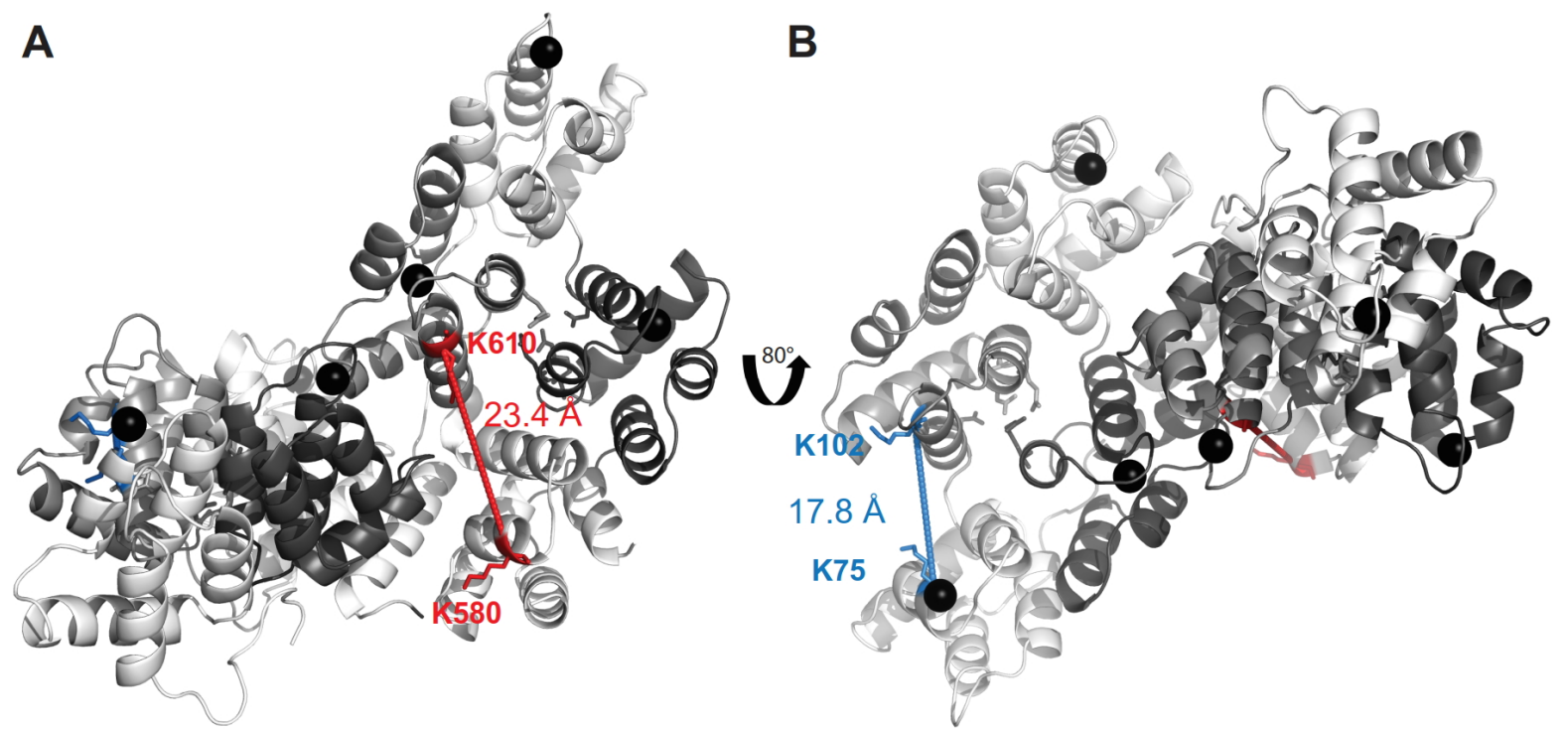

Figure 23: Significantly changed cross-links within annexin A6 (Anxa6). Cross-links from table 9 were plotted with pyM OL on a crystal structure of bovine Anxa6 (pdb: 1avc) ${ }^{159}$. A: a perpendicular view of lobe $A$, the central ion pore and its constituting amino acid residues (right and above the cross-link). B: View is rotated by $80^{\circ}$ along the vertical axis with the similar view of lobe B. Cross-linked lysine positions are indicated. Cross-links that had increased and decreased intensities in an excited state are depicted as red and blue lines, respectively. Bound calcium ions are depicted as black spheres, while separate annexin repeat domains are colored in different shades of grey.

Apart from I3PR, the $\mathrm{Ca}^{2+}$ stimulus applied in this study had an impact on two other proteins residing in the ER, i.e. calcium binding chaperone calreticulin ${ }^{162}$ and its co-chaperone endoplasmic reticulum resident protein 29 (ERp29) ${ }^{163}$. These significantly changed cross-links are further discussed in chapter 4.4.3. 


\subsubsection{Significantly changed cross-links in proteins, which do not directly bind calcium}

$M$ any significantly changed inter-links were observed among proteins that are not known to directly bind calcium ions or being involved in calcium signaling. Cross-links between alpha- and beta-subunits of tubulin and centractin, respectively, were more abundant in response to stimulation. With regard to a cross-link between alpha- und beta-spectrin that also increased in its abundance (figure 21), there is a common trend of higher abundant cross-links among cytoskeletal proteins under excited conditions. In addition, a cross-link between actin and spectrin beta chain was also quantified with a significant but minor 1.29 fold-change upon excitation (supplementary table 4). Thus, stimulation of synapses seemed to influence cytoskeletal proteins and their interactions among each other.

The cell cycle exit and neuronal differentiation protein 1 (Cend1) manifested a peculiar behaviour. All cross-links that exhibited a significant change were lower abundant upon stimulation (figure 24A). Unless the protein lost all secondary and tertiary structural components and linearized completely, every conformational change that departs two lysines should bring two other lysines closer to each other. Cend 1 was initially descibed as a regulator that promotes cell cycle exit of neuronal progenitors and neuronal differentiation ${ }^{164}$. In addition, it was found to regulate calcium-release from intracellular stores. Overexpression of Cend1 led to a decreased ATP-mediated calcium-release from inositol-1,4,5trisphosphate-sensitive stores, while the overall calcium concentration in the cytoplasm was unaltered ${ }^{165}$. A direct sensing or binding of calcium ions was not described, though. Cend1 is a small protein with one transmembrane domain sitting in the outer membranes of mitochondria, the endoplasmic reticulum, and plasma membrane. It was described as a homodimer with a disulfide bridge between cysteines $119^{166}$ (figure 24). A possible explanation for exclusively decreasing crosslinks could therefore be the dissociation of the dimer upon excitation as proposed in figure 24B. However, no cross-link between exactly the same residue on two separate molecules was detected. Another reason could be the binding of an interaction partner, which is again not supported by identified inter-links.
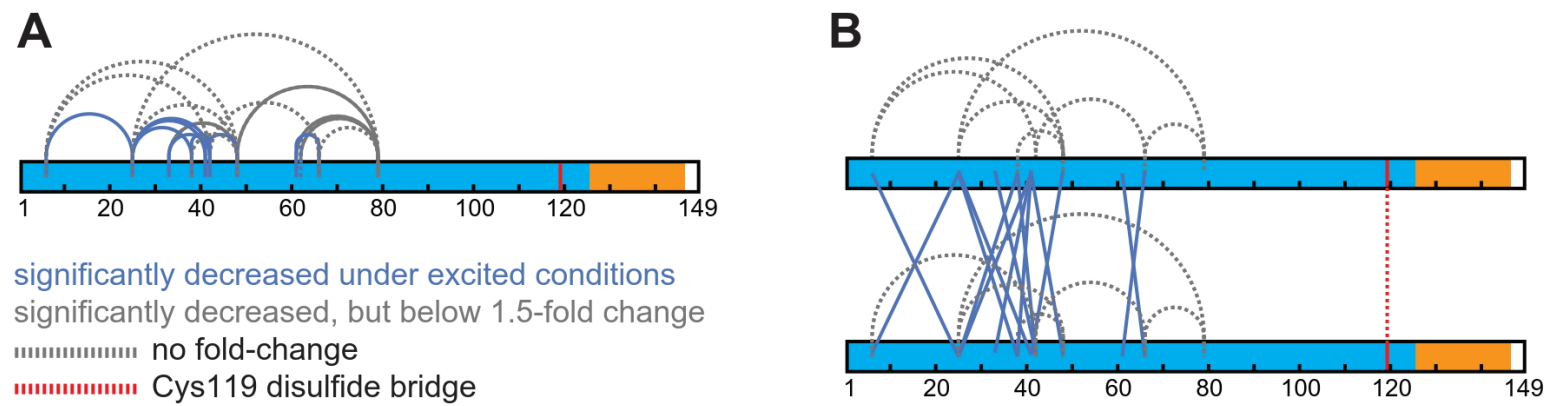

significantly decreased under excited conditions significantly decreased, but below 1.5-fold change ..............." no fold-change ."............ Cys119 disulfide bridge transmembrane domain cytoplasmic/ domain

Figure 24: Significantly changed cross-links under excited conditions within cell cycle exit and neuronal differentiation protein 1 (Cend1). A: N-terminal cytoplasmic domain is colored in blue, while the transmembrane domain is colored orange. (A) Significantly less abundant links within Cend1 upon stimulation are plotted as blue lines, significant links below 1.5-fold change are shown as grey lines and insignificant links are shown as grey dashed lines. B: A dissociating dimer is proposed, see text for explanation.

Similar to Cend1, disintegrin and metalloproteinase domain-containing protein 23 (ADAM 23) is an integral membrane protein with the single transmembrane domain at the C-terminus and the majority 
of the protein protruding into the extracellular region ${ }^{167}$. A dimer cross-link was identified for ADAM 23 between the same residue and peptide with a higher abundance in response to stimulation. ADAM 23 could therefore have a preferred dimer conformation in response to excitation.

At last, several cross-links had a higher abundance within or between chaperone proteins like heat shock protein 90 alpha, heat shock cognate $71 \mathrm{kDa}$ protein, heat shock protein $105 \mathrm{kDa}$, and mitochondrial stress-70 protein. As described in chapter 3.4.4.2, calreticulin and ERp29 were also chaperones in the endoplasmic reticulum that exhibited changes. Thus, the excitatory conditions applied in this study apparently have an effect on the chaperone system in several organelles and the cytoplasm in terms of protein folding and complex assembly and disassembly. No cross-links to respective substrate proteins were identified, though.

\subsubsection{Significantly changed cross-links within mitochondrial proteins}

Despite the efforts made to decrease the dominance of mitochondria in the cross-linking analysis, it was not possible to completely deplete proteins of mitochondrial origin as visible in Supplemental figure 4. Accordingly, several mitochondrial proteins were identified with significantly changed crosslinks in response to stimulation. One of these proteins was the barrel-like membrane pore VDAC-1 residing in the mitochondrial outer membrane and plasma membrane ${ }^{144}$. Two significantly decreased cross-links were identified for the excited state. One intra-protein cross-link between K28 and K236 decreased approximately two-fold, while the other between K236 and K274 was below a 1.5-fold change (figure 25). In the model depicted in figure 25B, the cross-links were either close to or were clashing with a loop leading to the N-terminal alpha-helix that is not part of the barrel consisting of beta-sheets. No clashes of cross-links with the peptide backbone were observed in the model of figure $25 \mathrm{~A}$, which indicates that the $\mathrm{N}$-terminal domain was adopting a conformation similar to the one shown in figure 25B during excitation, hence the cross-links had a lower abundance. The $\mathrm{N}$-terminus is rather flexible and is thought to be involved in channel gating depending on the membrane potential ${ }^{168}$. Interestingly, both models depicted in figure 25 presumably adopt an open conformation. Several other structural models of VDAC were reported in the past, all of which are similar to figure $25 \mathrm{~B}$ with the $\mathrm{N}$-terminal alpha-helix close to the pore wall. Ujwal and colleagues proposed that channel closure could be enabled by a movement of the alpha-helix towards the center of the pore lumen along a hinge in the loop close to the helix ${ }^{169}$. Such a state would still be incompatible with the cross-links observed here. The $\mathrm{N}$-terminus in figure $25 \mathrm{~A}$ does not clash with the cross-links, because the unstructured loop originating from the beta-sheet bends in the opposite direction compared to figure 25B and then traverses the pore centrally. In summary, the quantitatively changing cross-links upon excitation are probably due to a changed conformation of the $\mathrm{N}$-terminal domain of VDAC as a response to an altered membrane potential. Due to a so far missing structure of closed VDAC, no assessment is possible about the VDAC gating state in response to synaptic stimulation. 

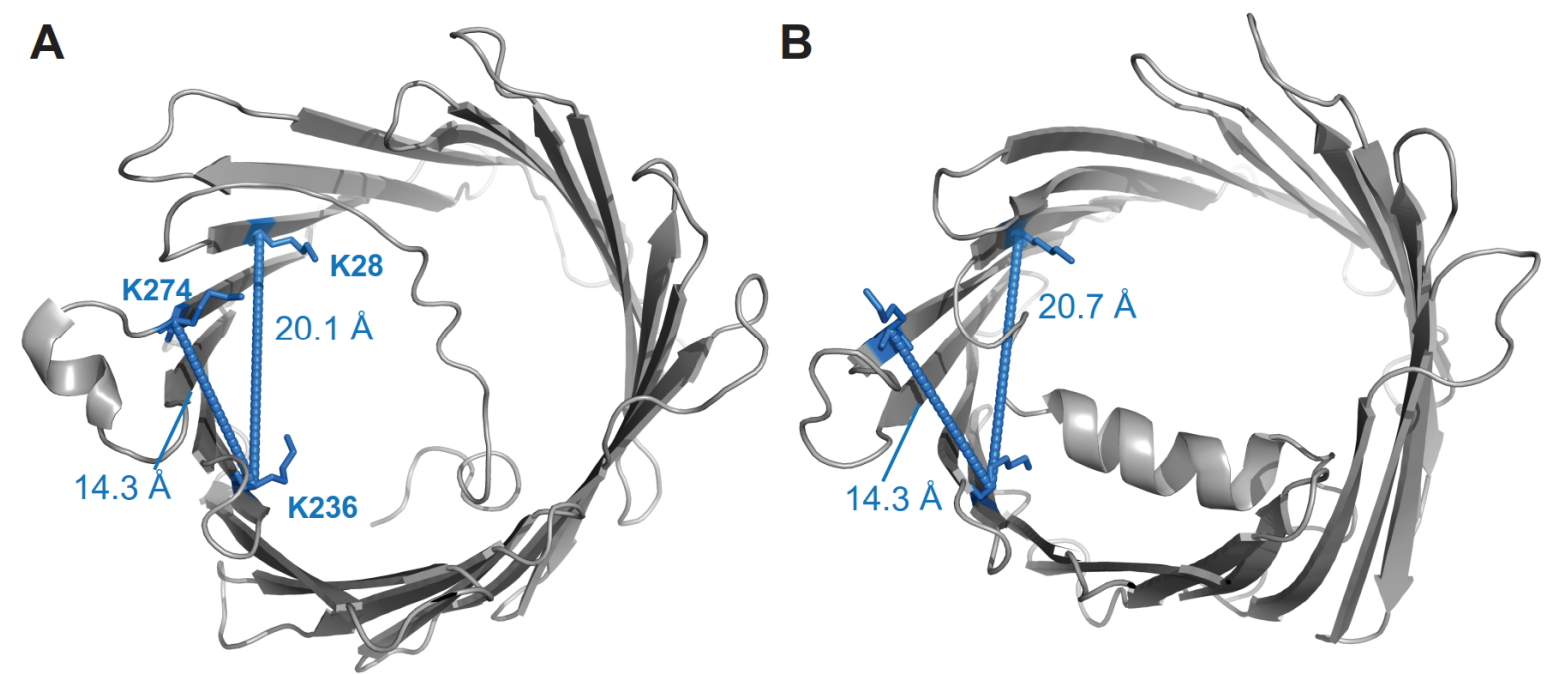

Figure 25: Significantly changed cross-links within voltage-dependent anion channel (VDAC). Cross-links from table 9 and the supplementary table 4 were plotted with pyM OL on an NM R- (A, pdb: 2k4t) ${ }^{170}$ and both NMR and X-ray based (B, pdb: 2jk4) 168 models of rat VDAC-1. Cross-linked lysine positions are indicated. Cross-links that had a decreased intensity in an excited state are depicted as blue line. The cross-link between lysine 236 and 274 was significant but exhibited a fold-change below 1.5.

Apart from transporting ions like calcium, VDAC is the central channel for ATP across the mitochondrial outer membrane ${ }^{171}$. Interestingly, two other mitochondrial proteins that exhibited cross-links with a significantly changed abundance upon stimulation were involved in ATP transport and metabolism, i.e. creatine kinase and ADP/ATP translocase 1/2. The latter exchanges ADP from the intermembrane space with ATP from the matrix, which then leaves the mitochondrium through VDAC and is available for all energy-dependent processes in the cell. Creatine kinase is a homooctameric complex that resides in the intermembrane space and transfers a phosphogroup from ATP onto creatine, thereby sustaining a high availability of ADP for both ADP/ATP translocase, as well as ATP synthase ${ }^{172}$. VDAC, ADP/ATP translocase and creatine kinase are therefore functionally linked to each other. A direct and indirect protein interaction was also described in the past ${ }^{173}$, but no inter-links were identified or quantified with significantly altered intensities. A significantly altered intra-link was also exhibited within the alpha subunit of ATP synthase and succinate dehydrogenase, which as components of the electron transport chain are directly involved in ATP generation.

\subsubsection{Proteins with no significantly changed cross-links}

Naturally, most intra- and inter-links did not change upon stimulation of synaptosomes. However, some type of intra-protein conformational change, complex assembly, or dissociation was expected for some proteins that are involved in synaptic transmission processes. After decade-long research efforts to understand the mechanisms behind transmitter release, the behaviour of many synaptic proteins in response to excitation is fairly well understood. Three examplary proteins are shown in figure 26 , for which several cross-links were identified but the ratio between excited and resting state was insignificant. As the protein name suggests, voltage-dependent L-type calcium channel is responding to a change in membrane potential by channel opening and calcium conduction. Although 
it is a calcium channel, no cross-link changes were observed as described for other channels in chapter 3.4.4.1.

Stxbp1/M unc18-1 is known to bind Stx1, lock it in a closed conformation and sequester it from its interaction partners of the SNARE complex. On the other hand, it can bind to the pre-assembled SNARE complex and promote vesicle fusion. The switch from a closed to an open conformation necessitates an extensive movement of the $\mathrm{H} 3$ domain in Stx ${ }^{88}$, which was not represented in the quantified crosslinks in the present study.

At last, the AP-2 complex is able to bind specific motifs on cargo proteins in the plasma membrane that derive from fused synaptic vesicles. M otif binding is accompanied by a major conformational change of the $\mathrm{C}$-terminal $\mu$ subunit. Once bound to its cargo and to the membrane in an open conformation, it can bind to the clathrin heavy chain and other scaffolding proteins to promote clathrin coat assembly and finally clathrin mediated endocytosis ${ }^{145}$. The endocytotic machinery must have been active during the applied experimental conditions, but no conformational changes within AP-2 complex were detected in the manner of significantly changed cross-links. 
A Syntaxin \& Syntaxin-binding protein

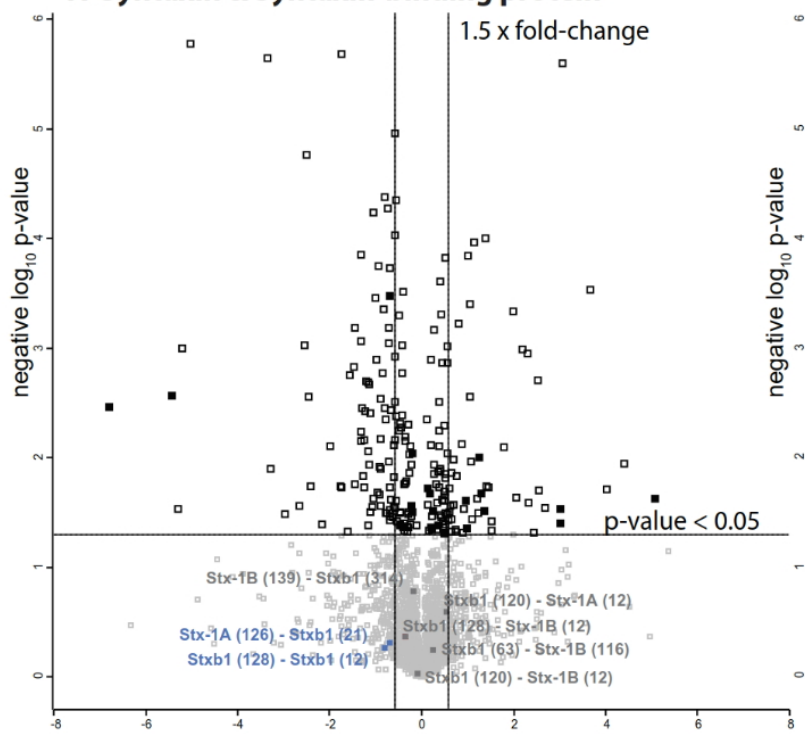

$\log _{2}$ ratio of unique cross-link sites, excited to resting state

C Voltage-dependent calcium channel

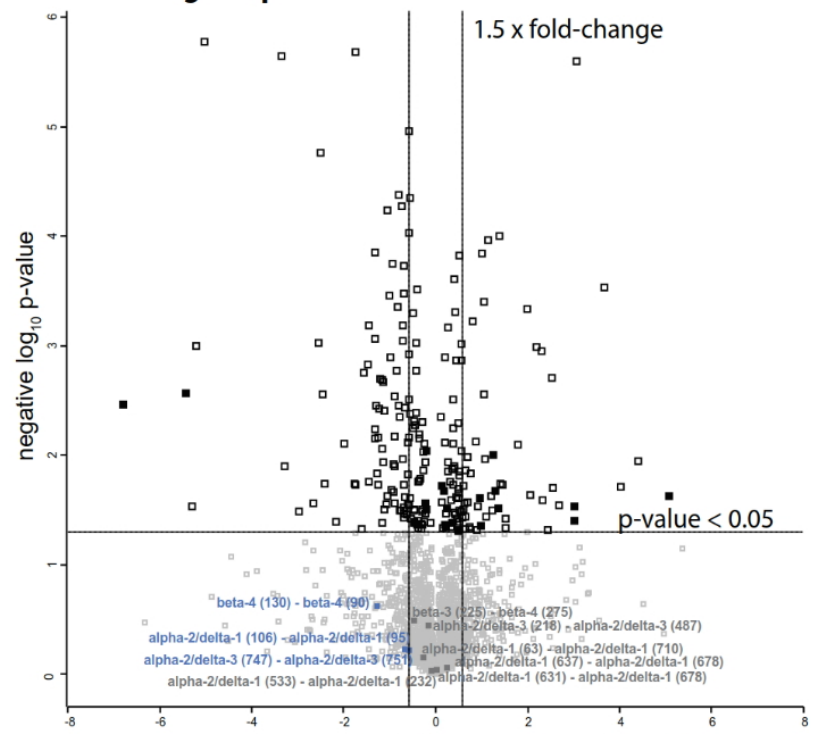

$\log _{2}$ ratio of unique cross-link sites, excited to resting state
B AP-2 complex

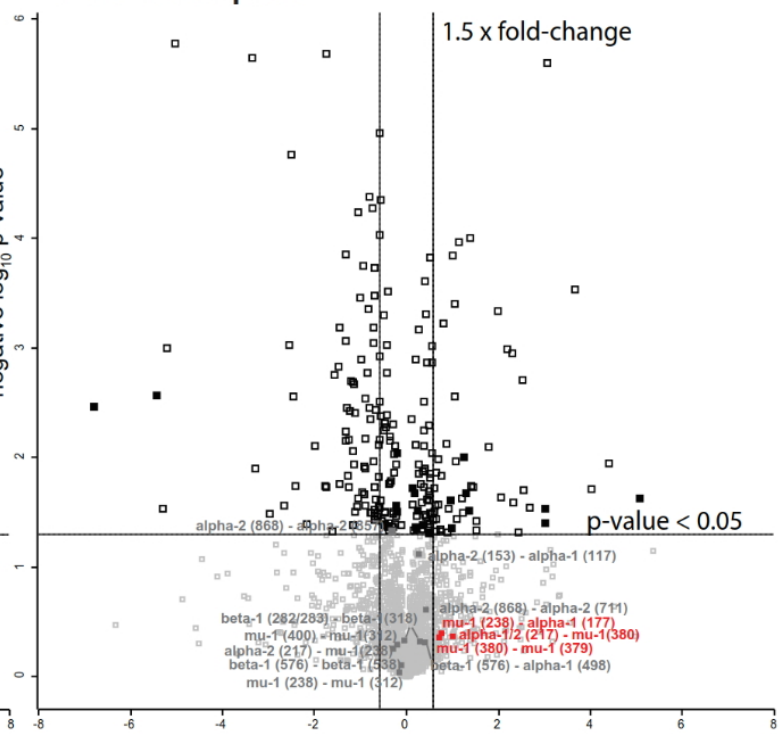

$\log _{2}$ ratio of unique cross-link sites, excited to resting state

Figure 26: Scatter plot of fold-changes of unique cross-link sites under excited conditions in comparison to resting conditions plotted against the negative $\log _{10} p$-value for three exemplary complexes that did not change or were insignificant. Synaptosomes were prepared from rat brains and cross-linked with isotopically labelled linkers in an excited and resting state, respectively (chapter 2.2.4.4). Samples were pooled and synaptosomal plasma membranes were enriched (chapter 2.2.4.5). Cross-linked peptides were analysed by LC-M S and identified with plink 1 at $1 \%$ FDR against a peptide database (chapter 2.4.3), followed by quantification with $\mathrm{XiQ}^{1}$ (chapter 2.4.4). Results from three bioreplicates and nine cross-linking replicates were merged, filtered for at least three valid values and a t-test was performed with a p-value cut-off below 0.05 (chapter 2.4.5). 


\section{Discussion}

The aim of this thesis was to identify protein interactions in the neuronal synapse by chemical crosslinking mass spectrometry (XL-MS) and to quantify changes in protein complex dynamics upon chemically evoked neurotransmission. Main challenges emerge from the $n^{2}$ problem, which is associated with the XL-M S analysis of complex samples. With increasing number of peptide sequences derived from a protein database, the search space for the identification of cross-linked peptide pairs increases exponentially. The $n^{2}$ problem effectively limits the analysis of complex samples to the most abundant proteins. In addition, proteomic analyses of synaptosomes are dominated by myelin and mitochondrial proteins. For this reason, a workflow was established to obtain a cleaner synaptosome fraction and to deplete mitochondria following the cross-linking reaction and synaptosomal lysis. Furthermore, a peptide-focused database search approach was developed to address current database search limitations in XL-MS. The new method overcomes the focus on the most abundant proteins and was validated with purified cross-linked complexes and bacteria as model systems. Taken together, the efforts led to the construction of an XL-MS based protein interaction map of the synapse. Furthermore, many significantly changing inter- and intra-protein cross-links were detected within, e.g. ion channels (SERCA, PMCA, I3PR) and $\mathrm{Ca}^{2+}$-binding proteins (Spectrin alpha, synaptotagmin, Annexin 6), upon chemical stimulation.

\subsection{Advantages and disadvantages of applying a peptide-focused database search approach for XL-MS}

Yilmaz and colleagues ${ }^{47}$ reasoned that algorithms tailored towards the analysis of non-cleavable crosslinked peptides are not reliable on a proteome-wide scale due to lack of robustness. Even if the time component of an exponential search space increase would be overcome computationally, the vast number of peptide candidates would likely lead to false positive identifications and thus less sensitivity and reliability ${ }^{47}$. The peptide-focused approach presented here is a biochemical strategy to overcome these problems. Instead of considering all theoretically cross-linkable peptides of an entire proteome, the approach focuses on those peptides, which were identified with a cleaved and alkylated crosslinker and were therefore proven to participate in a cross-link. Naturally, dead-end cross-links, i.e. the cross-linker reacted with one lysine residue and hydrolyzed on the other side of the molecule, are indistinguishable from cross-links after cleavage of the disulphide bridge and would contribute to the database. However, they were depleted to some extent for bacteria and synaptosomes by sizeexclusion chromatography prior to cleavage. The focus on peptides reduced the database search space by a factor of ten to 40 for bacteria and synaptosomes. The search space reduction allowed less chance for false positives by random matches, yielded more identified CSMs for large proteomes by an increased sensitivity, and took a fraction of search time at the same $1 \%$ FDR (false-discovery rate) cutoff (table 7 and table 8). In addition, it enabled the cross-linking analysis to move away from the most abundant proteins that are typically considered with non-cleavable cross-linkers and allowed the identification of cross-links to less abundant proteins. 
4.1 Advantages and disadvantages of applying a peptide-focused database search approach for XLMS

\subsubsection{Validation of the peptide-focused approach on purified cross-linked complexes}

The application of a peptide-focused approach on purified cross-linked complexes with known composition and structure enabled a straightforward performance comparison to a conventional database search approach. The latter is a database search against the sequences of proteins that are present in the sample. Model structures are available for yeast polymerase $\|^{125}$ (Pol II), transcription factor ${ } \mathrm{H}^{125}(\mathrm{TFIIH})$, human $20 \mathrm{~S}$ proteasome ${ }^{126}$ and transitional endoplasmic reticulum ATPase ${ }^{138}$ (P97). Cross-linked lysine residues identified with both approaches were mapped on model structures and the $\mathrm{C} \alpha-\mathrm{C} \alpha$ atom distance was measured. These distances provided an independent means to evaluate whether identified cross-links were physically feasible in the context of a static model structure, i.e. if cross-links did not exceed a distance of $30 \AA$. A comparison of high confident identifications with multiple CSM s (cross-link to spectrum matches) and/or a high pLink score derived from both approaches was performed (chapter 3.2, figure 3 and supplemental figure 1). Indeed, the number of cross-linked residues and the $\mathrm{C} \alpha$ - C $\alpha$ atom distance distribution was virtually identical with slight variations depending on the complex. It is concluded that the peptide-focused approach reports equally accurate identifications as the conventional approach and is therefore suitable for XL-MS analysis of cross-linked protein complexes as well as bacteria and synaptosomes.

More CSMs were identified for all complexes using a conventional database search approach. However, it was not expected that the peptide-focused approach would outperform the conventional approach, since the effect of the exponential search space increase is not significant with a database size of ten to fourteen proteins. After all, many algorithms for peptide cross-linking were developed to efficiently analyze protein complexes of up to 50 to 100 components $^{39,47,174}$.

\subsubsection{Generation of a peptide database}

The examination of the peptides, which were identified as cross-linked with the conventional but not the peptide-focused approach, granted the possibility to improve the peptide-focused workflow and to estimate its weaknesses. It is not possible to consider and identify cross-links to the protein $\mathrm{N}$ terminal amino group with the peptide-focused approach. The reason for this limitation was the fact that the search algorithm would interpret each provided peptide as a distinct protein and would consider a cross-link to its N-terminus, although it was not a possible cross-link site at the time of the reaction. Peptides, which were missing in the generated peptide database but were identified as crosslinked with the conventional approach, tended to be very short or very long. This unusual sequence length distribution was not accommodated by the default settings of common proteomics database search algorithms. Peptides below seven amino acids are usually excluded ${ }^{135}$, because they fragment poorly and often cannot be assigned to one single protein due to their non-unique sequence. An upper limit is applied on peptide length to decrease the search space. The distribution of lysines and arginines throughout proteins and the cleavage efficiency of trypsin leads to a low and negligible number of tryptic peptides above 35 amino acids. For example, only 3.2\% of all peptides are longer than 35 amino acids in an in silico tryptic digest of the rat proteome ${ }^{175}$. However, tryptic peptides cross-linked with an NHS-based cross-linker always contain at least one non-cleaved lysine residue, which reacted with the cross-linker, and are thus on average two times longer than regular tryptic peptides. Furthermore, 
cross-linked peptides are regularly identified with a long, well-fragmenting alpha and a short, poorly fragmenting beta peptide of four to six amino acids length that are true-positive hits.

Because of these two observations, the database search settings for peptide database generation were adjusted to accommodate short and long peptides. However, it only partially reduced the number of peptides, which were missing in the peptide database but were identified as cross-linked by the conventional approach. Apparently, some peptides could only be identified in the context of a crosslink. Their properties rendered them unfavorable for identification as a linear peptide and only the cross-linked peptide increased the probability of their identification. A similar observation was also made by Buncherd and colleagues ${ }^{176}$. Unfavorable properties likely involve peptide chromatographic behavior and ionization and fragmentation efficiency.

A bias was observed for the identification of very short peptides of four to five amino acids length. Due to their hydrophilicity, they might have failed to efficiently bind to $\mathrm{C} 18$ reverse phase material, which is used as stationary phase for desalting and liquid chromatography prior to MS analysis (LC-M S), and were lost. As mentioned above, such short peptides are very stable and fragment poorly, in addition to the limited number of fragment ions they can produce. Their identification as linear species was therefore difficult, whereas they were often identified cross-linked to long well-fragmenting peptides. Correspondingly, the identified cross-link was mostly based on fragment ions derived from the longer peptide, which affected the confidence of the identification negatively. On the other hand, very long peptides might have been too hydrophobic and were not eluted from the C18 material after desalting ${ }^{23}$. Only when cross-linked to a shorter peptide, the longer peptide was hydrophilic enough to be detectable by LC-M S. Similar to peptides with short sequences, peptides, which for example contain several proline residues, fragment inefficiently as a linear species but might be identified along with a peptide with more favorable MS properties cross-linked to it. At last, the cleaved cross-link tag consisting of one half of the DSP cross-linker and a carbamidomethyl group certainly had an influence on the ionization of the peptide.

The considerations listed above might have contributed to missing peptides, which were cross-linked under experimental conditions but were absent from the database of cross-linking peptide candidates. Therefore, the peptide-focused approach could not identify as many CSMs as the conventional approach. However, CSMs that were exclusively identified by the conventional database search approach and not by the peptide-focused approach had a low pLink score on average as described in chapter 3.2. The low score was probably due to their unfavorable properties regarding ionization and fragmentation efficiency mentioned above. While they constituted a significant fraction of identified cross-links for small protein databases, their proportion decreased with increasing target-decoy competition that accompanies a growing search space. Accordingly, with increasing search space the peptide-focused approach identified more CSMs and unique cross-linked residues for bacteria and synaptosomes than the conventional database search approach (table 7 and table 8).

The importance of a peptide database covering all peptides that were able to cross-link was underlined by the false-positive identification that was only identified with the peptide-focused approach for $20 \mathrm{~S}$ proteasome (figure 3). If a peptide involved in a cross-link was not present in the peptide database, the search algorithm either would not identify it or would report the second best and wrong hit with partial sequence similarity and a lower pLink score. Furthermore, a peptide database generated from two replicates of DSP cross-linked and cleaved Pol II identified $43 \%$ more CSM s than a peptide database generated from one replicate (chapter 3.2). It was therefore reasonable to perform and merge multiple DSP cross-linking replicates of synaptosomes to generate a near-complete peptide database. Indeed, a new subset of unique peptides was identified with each replicate as shown in 

MS

figure 10. With increasing number of replicates, less unique peptides were identified indicating that all putative cross-linking peptide candidates were assembled in a near-complete peptide database.

The peptide database size was very small in comparison to the number of theoretically cross-linkable peptides derived from the respective proteome (table 7 and table 8). Therefore, the question of undersampling arises, i.e. there were not enough target and decoy sequences in the peptide database to accurately calculate the false-discovery rate. However, due to exponential search space increase, tryptic peptide pairs originating from 50 proteins would already correspond to the entire linear peptide database search space of the human proteome ${ }^{36}$. In silico digestion of 50 proteins approximately generates 2,500 theoretically cross-linkable peptides, which is by far below the size of peptide databases used in this study for bacteria, as well as for synaptosomes. Thus, it is assumed that enough peptide candidates were present in the peptide database for an accurate target-decoy competition and FDR calculation.

Another strategy to ensure that the peptide database represents the peptides, which cross-link under the actual experimental conditions, as much as possible was to use thiol-cleavable DSP for both the cross-linking analysis and peptide database generation (chapter 3.2). Cross-linking with DSP and splitting one sample would circumvent the possible different cross-linker reactivity and any variations arising throughout the workflow. The random cross-linking depicted in figure 4 led to the abandonment of this approach. However, it is possible to minimize disulphide scattering by keeping the $\mathrm{pH}$ slightly acidic and/or by adding $\mathrm{N}$-ethylmaleimide to quench any reduced thiol groups that are contributing to the aforementioned scattering ${ }^{139}$. N-ethylmaleimide was used as alkylation reagent in the past to identify disulphide bridges on a proteomic scale ${ }^{177}$. The reagent is also membrane permeable and could therefore be used for ex vivo cross-linking of synaptosomes. However, reduced cysteines are often essential for enzymatic activity of proteins, for example cysteine proteases or most prominently the $\mathrm{N}$-ethylmaleimide sensitive factor NSF that cannot disassemble SNARE complexes after treatment with $\mathrm{N}$-ethylmaleimide ${ }^{178,}{ }^{179}$. Therefore, DSP cross-linking of synaptosomes in presence of $\mathrm{N}$-ethylmaleimide will probably negatively affect the excitability of synaptosomes and impede one of the major aims of this thesis, which is capturing conformational changes upon stimulation.

A disadvantage of the presented approach is the necessity to generate a peptide database in parallel to the actual cross-linking experiment, which means additional work in terms of sample preparation, LC-M S acquisition, and data processing. In addition, it has to be generated separately for each sample type and is limited to cross-linkers with a thiol-cleavable and non-cleavable form. However, as soon as the peptide database is generated from several cross-linking replicates for a specific sample type like synaptosomes, it is presumably suited for all forthcoming experiments on that particular sample type and does not necessitate a DSP cross-linked replicate in parallel each time (figure 10). In addition, comparative studies in the past have shown that NHS-ester based reactivity towards primary amines and a cross-linker comprised of six carbon atoms, as is the case for DSS, are the most successful in terms of identifications ${ }^{27}, 180$. Therefore, a focus on the chemical cross-linkers DSP and DSS for the peptide-focused approach is well motivated.

The principle of search space reduction by using peptides with known engagement in cross-links can also be expanded further. Other strategies for peptide database generation could be tested, for example NHS-acetate for tagging of lysines that are solvent-accessible. Other thiol-cleavable and noncleavable cross-linker pairs are possible, as well as cross-linkers with other cleavage chemistries, for example the above mentioned bis-(succinimidyl-)-3-azidomethyl glutarate, MS-cleavable DSSO, or disuccinimidyl tartrate that is cleavable by sodium periodate. The principle is also extendable to applications beyond XL-M S. For example, a peptide database could be used for the identification and 
quantification of post-translational modifications that were identified in previous experiments. Similar to XL-MS, the consideration of multiple post-translational modifications increases the search space, which is accompanied by decreased sensitivity. Therefore, using a peptide-focused approach for the identification of known post-translational modifications on these peptides might be helpful to increase sensitivity.

\subsubsection{Comparison of the peptide-focused approach to alternative strategies for database search space reduction in XL-MS}

The usage of an M S-cleavable cross-linker like DSSO in connection with the software XlinkX ${ }^{48,49}$ enables the separate identification of peptides in a cross-link. During fragmentation in the collision cell, the breakage of the cross-linker leads to characteristic reporter ion pairs that correspond to the linear peptides and enable the calculation of the linear peptide mass. By selecting the cleaved peptide ions in MS2 for an MS3 fragmentation event, linear peptides can be identified independently from their respective cross-linked peptide pair. The $n^{2}$ problem of XL-MS is converted to a $2 n$ problem thereby facilitating whole-proteome cross-linking and analysis ${ }^{48,49}$. An approach based on MS-cleavable crosslinkers is more straight-forward than the approach presented here, because it does not necessitate parallel experiments to generate a peptide database. However, the approach relies on the presence of high-abundant reporter ion pairs of cleaved cross-linked peptides in the M S2 spectrum to calculate the mass of the linear peptides, which are not always present ${ }^{181}$. In addition, efficient identification of cross-linked peptides benefits from complementary fragmentation strategies including collision induced dissociation on MS2 and MS3 level as well as electron transfer dissociation. However, an advanced "Tribrid" mass spectrometer (a tripartite hybrid mass spectrometer consisting of a quadrupole, linear ion trap, and an orbitrap $)^{182}$ is necessary with capabilities for sequential ion selection and fragmentation $\left(\mathrm{MS}^{n}\right)$ as well as precursor fragmentation with electron transfer dissociation. Furthermore, the time spent on the analysis of each precursor during LC-M S acquisition is very long, which impairs sensitivity that is crucial for the analysis of complex samples ${ }^{49,51}$. Therefore, a strategy based on M S-cleavable cross-linker was not utilized in this thesis. Another reason was the difficulty to quantify cross-links obtained with DSSO, because the quantification of changing cross-links and interactions was one major goal of this thesis. Although a deuteration strategy was reported for an MS-cleavable analogue of DSSO, termed DMDSSO, so far it is not readily available by commercial suppliers ${ }^{183}$.

During the course of the thesis, an approach for the quantification of MS-cleavable DSSO cross-links was presented ${ }^{59}$. It is based on TMT labelling ${ }^{21}$ and was benchmarked on relatively simple protein mixtures ${ }^{59}$. However, its applicability on more complex samples might be limited by the need for sample fractionation and cross-link enrichment. Since TMT labelling is usually performed as one of the last steps prior to LC-MS analysis, variabilities introduced during sample processing or cross-link enrichment could impair quantification.

Similar to the approach presented in this thesis, two other strategies were described in the past that reduced the search space for cross-linking analysis by using prior knowledge about peptides being prone to cross-linking. The first one is the computational approach of SIM-XL developed by Lima and colleagues $^{184}$ that employs a dynamic database reduction step. A preliminary search for dead-end cross-links is performed, followed by a cross-linking search considering peptides identified with dead- 
end cross-link and all peptides containing a reactive residue, e.g. a lysine in case of DSS ${ }^{184}$. One disadvantage of this approach is that often not all cross-linked peptides are identified with a hydrolyzed dead-end cross-link. A possible reason might be the presence of two very accessibly positioned lysines on the surface of a protein that are almost always cross-linked together. These reactive residues would lead to high abundant cross-linked peptide products but would not be identifiable by the approach of SIM -XL. In addition, LC-M S acquisition settings for cross-linked samples are optimized for higher masses and charges, which might lead to a low number of identified deadend cross-links. In case of the peptide-focused approach, peptide candidates are generated from actual cross-links that are cleaved and the analysis is performed in a separate experiment, which is optimized for the identification of linear peptides.

The other strategy was developed by Buncherd and colleagues ${ }^{176}$. Protein samples are cross-linked with bis-(succinimidyl-)-3-azidomethyl glutarate, proteolytically digested, fractionated by SCX chromatography and obtained fractions are incubated with a reducing agent. By reduction with tris(2-carboxyethyl)-phosphine, the azido group of the cross-linker is either reduced to a primary amine, or the cross-link is cleaved to an unchanged peptide $A$ and peptide $B$ containing the remainder of the cross-linker as a lactone group. Subsequently, the SCX fraction is submitted to another SCX chromatography run. Reduced cross-linked peptide pairs have a higher, while cleaved peptides have a lower charge state and elute at different time points in comparison to the first SCX run. Finally, cleaved peptides are identified and are taken as input for the cross-linking analysis of intact cross-links ${ }^{176}$. The approach of Buncherd et al. ${ }^{176}$ is very similar to the peptide-focused approach but it has the advantage that peptide search candidates are obtained from the same sample and thus, represent the crosslinked peptides optimally. However, generation of peptide candidates by cross-link cleavage happens at the expense of actual cross-links: Either the yield of cross-links is high, and the number of peptide candidates is low, or vice versa. Furthermore, their approach depends on two SCX chromatography runs, which are sources for sample loss, to distinguish between cross-links and cleaved linear peptides in addition to a custom analysis pipeline. In contrast, the peptide-focused approach is compatible with any fractionation method and almost any cross-linking database search algorithm and is therefore more versatile.

\subsection{In vivo XL-MS analysis of bacteria}

The purpose of in vivo cross-linking of Bacillus subtilis and cereus cells was to examine the performance of the peptide-focused approach on a complex system.

In order to obtain more biological conclusions from cross-linked bacteria, sample preparation could have been further optimized to increase identification numbers, e.g. by using more material and improve protein extraction. Nonetheless, many inter-protein cross-links between known protein interaction partners were identified (supplemental figure 2). In addition, many inter-protein cross-links were identified between proteins, which were hitherto not known to interact, but an interaction is coherent in the context of the respective protein function (supplementary table 1). A focus was placed on cross-links obtained between ribosomal subunits and elongation factor Tu (EF-Tu, figure 5).

The latter exhibited cross-links to ribosomal subunits RL14, RL17 and RL19 that are proximal to EF-Tu when it is bound to the aminoacyl-tRNA in the A site. However, multiple cross-links in one or both species, i.e. Bacillus subtilis and cereus, were identified between EF-Tu and RS5, RS8, and RS9 that 
reside at the bottom of the ribosomal small subunit $(30 \mathrm{~S})^{143}$. Interestingly, such a localization of EF-Tu was predicted before the emergence of near-atomic crystal and cryo-EM structures of the ribosome. Utilizing immunoelectron microscopy, i.e. antibody binding to its epitope within a large complex and visualization by negative staining electron microscopy, Langer et al. ${ }^{185}$ pinpointed the position of EFTu to the cytoplasm-exposed 'neck' region of the $30 \mathrm{~S}$ subunit far off the A site. These findings are in agreement with the cross-links observed in this thesis. The positioning was even functionally connected to subunit RS5, besides RS4 and RS12 ${ }^{185}$. Another study by Girshovich and colleagues ${ }^{25}$ employed the same technique in combination with cross-linking of $70 \mathrm{~S}$ ribosomal particles. They also placed EF-Tu to the exterior and frontal side of the $30 \mathrm{~S}$ subunit but closer to the interface between the $30 \mathrm{~S}$ and $50 \mathrm{~S}$ ribosomal subunits. Besides antibody binding to the $30 \mathrm{~S}$ subunit, they have observed binding to the base of the L7/L12 (RL7) stalk of the $50 \mathrm{~S}$ subunit ${ }^{25}$. Therefore, their proposed placement of EF-Tu to a certain extent agreed with contemporary atomic structures as shown in figure $5^{143}$. The reason for the different placement of EF-Tu could be the use of different samples. Langer and colleagues $^{185}$ have examined only the $30 \mathrm{~S}$ subunit, whereas Girshovich and colleagues ${ }^{25}$ utilized $_{70 \mathrm{~S}}$ ribosomes stabilized by cross-linking. They could therefore observe the association of EF-Tu with both the $30 \mathrm{~S}$ and $50 \mathrm{~S}$ subunit. A possible explanation might be that EF-Tu is binding the bottom side of the 30 S subunit while the latter is not bound by the 50 Slarge subunit. Thus, EF-Tu could have a functional role at this binding position in a non-assembled ribosome only. Langer and colleagues $^{185}$ also experienced that the complex consisting of the $30 \mathrm{~S}$ subunit, mRNA, EF-Tu and aminoacyl-tRNA was unstable with up to $70 \%$ of the complex dissociating during sucrose gradient centrifugation ${ }^{185}$. Such a transient interaction, which is only present in subcomplexes of a ribosomal preparation, could have been missed in subsequent structural studies of the ribosome. However, the in vivo cross-linking performed in this thesis could have been able to trap such transient interactions in a subcomplex, since no artificial conditions are applied apart from the washing steps after bacterial cultivation.

Recently, de Jong and colleagues ${ }^{141}$ have cross-linked Bacillus subtilis cells in vivo. This thesis has reported significantly more inter- and intra-links than de Jong et al. ${ }^{141}$, but both studies differ in terms of sample preparation, number of replicates, LC-M S instrumentation, and data analysis. Similar to this thesis, the majority of cross-links was identified within transcriptional and translational complexes, which was expected for exponentially growing bacteria. However, they have not observed such a high interconnectivity of EF-Tu, especially to the bottom of the $30 \mathrm{~S}$ subunit ${ }^{141}$. This is probably due to the different cross-linker properties and maybe a lower sensitivity of their approach, since more crosslinks were identified in this thesis.

\subsection{XL-M S analysis of purified and functional synaptosomes}

\subsubsection{Depletion of mitochondrial and myelin proteins for a stronger focus on synaptic proteins}

Due to the focus of this thesis on the XL-MS analysis of synaptic proteins, mitochondrial and myelin proteins were considered as contaminant proteins. However, signal transmission between neuronal cells is an energy-demanding process, therefore ATP-producing mitochondria are an essential 
component the presynapse. They provide energy for processes like, among others, maintenance of plasma membrane potential, synaptic vesicle priming, endocytosis and neurotransmitter loading ${ }^{68}$. Proteomic analyses of synaptosome preparations are therefore dominated by proteins of mitochondrial origin. Wilhelm and colleagues ${ }^{120}$ characterized synaptosome preparations by electron microscopy and estimated the intra- and extra-synaptosomal mitochondrial fraction of total material to 10 and $20 \%$, respectively. By determining absolute copy numbers of proteins, they estimated the fraction of mitochondrial proteins to $25 \%$. In addition, approximately $10 \%$ of observed structures in electron microscopic analyses of synaptosome preparations were assigned to myelin and myelin fragments. Myelin proteins therefore represented $3.45 \%$ of total protein in synaptosome preparations ${ }^{120}$.

Correspondingly, synaptosomal subfractions obtained by biochemical purification are also contaminated by mitochondrial proteins. As an example, Boyken and colleagues ${ }^{130}$ performed a relative quantification study of docked and free synaptic vesicle fractions from glutamatergic and GABAergic synapses. $45 \%$ of all abundance ratios were obtained for mitochondrial proteins, which indicated that mitochondria were unintentionally co-purified during immunoisolation of synaptic vesicles ${ }^{130}$. More than LC-M S based proteomics, XL-MS is challenged to detect low-abundant crosslinking products that occur sub-stoichiometrically during the cross-linking reaction. For example, in an analysis of the mitochondrial cross-linking interactome with DSSO and an unbiased whole proteome database search, $60 \%$ of all identified cross-linked residues were found within the $10 \%$ most abundant proteins $^{52}$. The dominance of the most abundant proteins observed for purified mitochondria exemplifies the sensitivity issue that XL-M Sfaces. It was therefore critical to deplete mitochondrial and myelin proteins in this thesis. Cross-linked peptides originating from myelin and mitochondrial proteins compete with cross-linked peptides from synaptic proteins for detection during LC-MS analysis. As shown in figure 6 and figure 7, contaminant proteins were successfully depleted by additional purification steps, which also reduced the number of CSM $s$ identified within these proteins (chapter 3.4.1.1 and 3.4.1.2). Myelin proteins were depleted from intact synaptosomes four- to 16-fold by continuous sucrose density gradient centrifugation (figure 6). M itochondrial proteins were depleted two- to four-fold by discontinuous sucrose density gradient centrifugation with a single $1.05 \mathrm{M}$ sucrose cushion after synaptosome cross-linking and lysis (figure 7B, right). To monitor depletion, the sucrose concentration for density gradient centrifugation was lowered step-wise from $1.2 \mathrm{M}$ to $1.05 \mathrm{M}$ sucrose. Cushions with concentrations below $1.05 \mathrm{M}$ sucrose were also tested but did not enable a stronger depletion of mitochondrial proteins (data not shown). A possible explanation for the comigration of mitochondrial with synaptic proteins after cross-linking observed in figure 7A could be the localization of mitochondria within synapses. Electron tomography experiments have shown that a subset of mitochondria resided close to active zones and were anchored to the synaptic membrane by cytoskeletal structures. Furthermore, this anchor was interconnected with the cytoskeleton of the synapse, which also tethered synaptic vesicles close to the active zone ${ }^{186}$. It is therefore conceivable that this anchored subgroup of mitochondria was covalently cross-linked to synaptosomal membrane proteins after cross-linking and co-migrated in subsequent purification steps. Nonetheless, the two implemented purification steps significantly reduced contaminant myelin and mitochondrial proteins and thereby enabled a stronger focus on cross-linked synaptic proteins. 


\subsubsection{The protein interaction network of functional synaptosomes captured by XL-MS}

The purification workflows for the depletion of myelin and mitochondrial proteins were applied in combination with the peptide-focused approach for the XL-MS analysis of cross-linked synaptosomes. In total, eleven cross-linking reaction replicates corresponding to four biological replicates of resting and excited synaptosomes were analyzed together and identified cross-linked residues were combined.

Based on these identified cross-linked residues, an interaction map was constructed covering proteins that are involved in neuronal signal transmission (figure 12), membrane channels and receptors (figure 13), and cell adhesion and cytoskeleton proteins (figure 14). As shown in figure 12 and figure 13, XLMS was able to primarily capture stable protein interactors like multi-subunit complexes, e.g. AP-2 complex, $\mathrm{Na}^{+} / \mathrm{K}^{+}$-transporting ATPase, V-type proton-transporting ATPase, Camkll, and GABA receptor. In addition, cytoskeletal proteins that can form stable interactions cross-linked strongly in between each other, e.g. spectrin, septin and tubulin subunits, interactors of actin, and cell adhesion molecules surrounding contactin 1 (figure 14). Among others, spectrin alpha and beta chain, actin, and ankyrin-2 cross-linked to different membrane channels suggesting a role for these cytoskeletal proteins in localizing channels to specific areas of the membrane (figure 12). Spectrin and ankyrin were described to immobilize voltage-dependent $\mathrm{Na}^{+}$channel ${ }^{187}$ in the membrane and bind to $\mathrm{Na}^{+} / \mathrm{K}^{+}$-transporting ATPase ${ }^{188}$ in the past, but not to PM CA. Globular non-polymerized actin was also found to bind $\mathrm{Na}^{+} / \mathrm{K}^{+}-$ transporting ATPase ${ }^{189}$.

However, several proteins that are important components of the synapse and are known to interact were not identified with inter-protein cross-links among each other. Most prominently, the subunits of the SNARE complex consisting of syntaxin-1 (Stx1), synaptobrevin/VAM P2, and synaptosomalassociated protein 25 (Snap25) were not cross-linked together ${ }^{86}$. However, they cross-linked separately to accessory regulators, e.g. Stx1 to Stxbp1/M unc18-1 ${ }^{190}$, and Snap25 to complexin-2 ${ }^{191}$ (figure 12). Further known interacting proteins, for which no inter-protein cross-links were identified, include pre-synaptic active zone-specific scaffold proteins RIM, M unc13, RIM-BP, ELKS, ERC2, and Liprin-1 $\alpha^{80}$. Only two known interacting proteins belonging to the active zone scaffold complex were identified with inter-protein cross-links, i.e. bassoon and piccolo and CASK and Lin-7B (also known as vertebrate homolog of Lin-7, Veli) (figure 12). Bassoon and piccolo have a redundant role in vesicle clustering at the synapse ${ }^{81}$ and were shown to co-localize ${ }^{192,}{ }^{193}$. CASK and Lin-7B form a complex together with Mint1 and were proposed to have a bridging role between cell adhesion and synaptic vesicle exocytosis by binding to both Stxbp1 and cell adhesion molecule neurexin ${ }^{85}$. In addition, the CASK-Mint1-Lin-7 complex is also important for potassium channel localization ${ }^{194}$, and neurotransmitter release ${ }^{195,196}$. The absence of inter-protein cross-links between the majority of active zone scaffold proteins was probably due to their low abundance. For example, in LC-M S based labelfree quantification analyses performed within the course of this thesis, M unc13, RIM, and RIM-BP regularly ranked at positions around 1,500 relating to abundance. They often failed to be quantified due to the identification of a single peptide only or were not identified at all (data not shown). The additional discontinuous sucrose gradient after synaptosome lysis could be a reason for the absence of the aforementioned proteins, since e.g. Munc-13 was significantly depleted 1.5-fold (figure 8). Although exhibiting a significant depletion (figure 8), piccolo, bassoon and complexin-2 were identified with inter-protein cross-links in the subsequent cross-linking analysis. The size of the protein might play a role whether inter-protein cross-links were identifiable, since large proteins often give rise to 
more tryptic peptides and contain more lysines that can cross-link. For example, bassoon and Piccolo have a size of 420 and $550 \mathrm{kDa}$ according to uniprot (www.uniprot.org).

Regarding the lack of a cross-linked SNARE complex, one has to consider that only a subset of synaptic vesicles is docked to the active zone and primed. Therefore, the number of vesicles ready for calciumdependent fusion is less than the total number of vesicles. Accordingly, only a minority of SNARE subunits would be present in a primed, pre-assembled, pre-fusion state consisting of Snap25, Vamp2, Stx1, Syt1, and complexin as suggested by Zhou and colleagues ${ }^{90}$. After synaptic vesicle fusion, fully zippered SNARE complexes are quickly bound by NSF and soluble NSF attachment proteins (SNAPS) and are disassembled within five to six seconds in vitro ${ }^{197}$ and probably much faster in vivo. In contrast, cross-linking of protein samples is usually performed in the time range of tens of minutes, e.g. crosslinking of synaptosomes was performed for $10 \mathrm{~min}$. Although the molecular reaction itself is sufficiently fast to capture assembled SNAREs, one needs to consider that the cross-linker takes time to diffuse from the buffer into the organelle and the reaction products have to accumulate to pass the detection limit of the mass spectrometer. In the meantime, the cross-linker shuts down the metabolism and fixates proteins in the respective conformation at the time of the cross-linking reaction. Therefore, only a minor fraction of SNARE proteins would be captured in an assembled SNARE complex.

In this line of arguments, other protein interactions that regulate synaptic functions at the spatiotemporal scale are only transient and might be difficult to capture by XL-MS. Examples are among others: binding of adaptor proteins (AP-2, stonin, epsin and AP180) to vesicular proteins (syt1, vesicular glutamate transporter) as well as clathrin for clathrin coat nucleation, interaction of actin and dynamin with myosin $\mathrm{VI}$ and $1 \mathrm{E}$ for vesicle fission, and disassembly of the clathrin cage by $\mathrm{Hsc} 70^{94}$. However, if only a small subset of a particular protein species was involved in protein interactions or the interaction is only short-lived, the number of respective inter-protein cross-links would be substoichiometric. Accordingly, the resulting tryptic cross-linked peptides would be low abundant. Their detection would be challenging by LC-M S because of a vast excess of competing linear peptides and cross-linked peptides, which were derived from intra- and inter-protein cross-links of stable complexes.

The question, whether cross-linker reaction kinetics are sufficiently fast to capture changing protein interactions during synaptic vesicle exocytosis is further discussed in chapter 4.4 and 4.4.7.

A more extensive fractionation could aid in identifying low-abundant cross-links, e.g. a second dimension of peptide fractionation by strong cation exchange (SCX) chromatography prior to peptide size exclusion chromatography (PSEC) (chapter 3.4.1.3). One replicate of cross-linked and mitochondria-depleted synaptosomes was submitted to additional cross-linked peptide fractionation by polymeric SCX chromatography. Fractions were eluted in two-steps with increasing concentration of methanol and ammonium acetate and were submitted to PSEC and LC-MS. The additional fractionation led to the detection of three times more unique cross-linked residues than PSEC alone (figure 9). A three- to four-step elution would have been performed for all replicates, if the protein yield of synaptosomal membrane preparations had been consistently high. Further scale-up and optimization of the fractionation protocol could enable a deeper proteomic coverage of cross-linked proteins in the synapse in the future.

Another reason for the lack of cross-links between proteins known to interact could be an unfavorable cross-linker chemistry. Interacting proteins might not have lysine residues on both partner proteins in close proximity to each other so that DSS could covalently cross-link them together. A cross-linker with an alternative reaction specificity and spacer length could be able to capture protein interactions that were not identified in this thesis. For instance, additional structural data was obtained for single proteins and purified complexes with the use of multiple cross-linkers in the past ${ }^{27,}{ }^{180}$. Furthermore, 
the Bruce and Heck lab have performed independent cross-linking interactome studies of mice mitochondria ${ }^{52,198}$. The latter used M S-cleavable DSSO with a spacer length of $10.3 \AA$, while the former used a trifunctional cross-linker called protein interaction reporter with a flexible cross-linker that can span up to $43 \AA^{199}$. Both cross-linker have the same reactivity, but the overlap of identified cross-linked residues between both studies was surprisingly low indicating that data obtained with both crosslinkers is complementary to each other ${ }^{52}$.

However, a complementary cross-linker would require the availability of a thiol-cleavable analogue to be fully utilizable with the peptide-focused approach. In addition, an isotopically labelled version of the cross-linker is necessary to enable a quantitative analysis of changing cross-links upon stimulation.

\subsubsection{Novel protein interactions captured by XL-MS}

A multitude of inter-protein cross-links were identified indicating previously unknown and compelling protein interactions (figure 12, figure 13, figure 14). Hereafter, some examples of cross-linked proteins were chosen and discussed in detail.

\section{Pyruvate kinase and ATPases}

The ATP-generating glycolytic enzyme pyruvate kinase cross-linked to ion channel subunits of $\mathrm{Na}^{+} / \mathrm{K}^{+}-$ transporting ATPase and V-type proton ATPase (figure 12, and figure 13). These inter-protein crosslinks implied that the energetic coupling of ATP generation and ion transport across membranes was facilitated by spatial proximity and even protein interactions. By measuring ATP consumption and mitochondrial respiration in cardiomyocytes, it was shown that $\mathrm{Na}^{+} / \mathrm{K}^{+}$-transporting ATPase exclusively used ATP generated from glycolysis, especially pyruvate kinase, and not from oxidative phosphorylation ${ }^{200}$. Furthermore, earlier studies ${ }^{201}$ showed an enrichment of glycolytic enzymes glyceraldehyde 3-phosphate dehydrogenase and 3-phosphoglycerate kinase in synaptic vesicle preparations. This enrichment was functionally connected to the proton gradient-driven glutamate uptake enabled by V-type proton ATPase ${ }^{201}$. No participation of pyruvate kinase was described for ATPsupply to V-type proton ATPase, though. The cross-links observed in this thesis agree with the functional coupling of glycolytic ATP generation and ATP-dependent ion channels described in the past ${ }^{200,} 201$ and provide evidence for a direct protein interaction. Such an interaction would bring ATP generation and consumption in close spatial proximity and facilitate efficient transporting processes of ions across membranes.

Septin-7 and nucleotide-binding proteins

Interestingly, Septin-7 displayed cross-links to two uncharacterized proteins, a zinc finger domain containing protein and a ribosomal protein L30-like, both of which possess a nucleic acid binding function according to uniprot (www.uniprot.org). Septin-7 is associated with presynaptic membranes ${ }^{202}$, localizes at the base of dendritic spines and is important for dendrite branching and morphology ${ }^{203}$ but was also found in synaptic vesicle preparations ${ }^{75}$. It is a matter of an ongoing debate, whether there is local translation at synapses and how transcripts, ribosomes and translation factors are transported to synapses and dendrites ${ }^{204,205}$. The cross-links observed in this thesis could be a result of septin-7-mediated localization of these nucleic acid-binding proteins to axons and dendrites and enabling a local translation of bound mRNAs. For example, a zinc-finger domain 
containing protein was found to bind and transport mRNAs on endosomes in fungal hyphae, which renders local translation of proteins at the tips of hyphae ${ }^{206}$. However, this protein complex could have been co-purified during synaptosome preparation and could thus be a contaminant. After all, septin 7 is not exclusively localized in dendrites but is also involved in cytokinetic processes like e.g. meiosis ${ }^{207}$. Therefore, it is difficult to draw any conclusions based on these findings.

\section{Contactin and Lgi1}

Leucine-rich glioma-inactivated protein 1 (Lgi1) was identified with cross-links to contactin 1 and 2, which implied an interaction that was not described before. Lgi1 is a secreted protein that is known to form a tripartite complex with ADAM 22 and postsynaptic density-95 protein and to regulate the number of postsynaptic glutamate receptors that are expressed on the surface ${ }^{208}$. M utations in genes encoding for Lgi1 and ADAM 22 are associated with epilepsy ${ }^{208}$. An interaction between the two proteins was also identified by cross-linking in this thesis (figure 14). Contactin is a cell adhesion molecule that heterodimerizes with contactin-associated protein in a cis-formation and is mostly known to anchor myelin sheets to the axon in the paranodal region of a myelinated axon ${ }^{209}$. An interaction was also verified by identified cross-links (figure 14). In addition, contactin is also expressed in both dendrites and axons of inhibitory interneurons. Accordingly, the phenotype of a mutant mouse strain lacking contactin exhibited less dendrites and aberrant axon guidance and dendritic projections. Therefore it was proposed that contactin promotes contacts between dendrites and axons during synaptogenesis ${ }^{210}$. Since Lgi1 is secreted and binds ADAM 22 on the postsynaptic side and contactin is expressed on the extracellular side of both dendrites and axons, an interaction of these two proteins is spatially possible and might have a functional relevance.

\section{Ndrg and ATPases}

$\mathrm{N}$-myc downstream regulated gene ( $\mathrm{Ndrg}$ ) protein 2 and 3 cross-linked to two alpha subunits of $\mathrm{Na}^{+} / \mathrm{K}^{+}$ ATPase as well as to PM CA (figure 13). Ndrg 2 transcription was found to be suppressed in glioblastoma cells and its overexpression reduced proliferation ${ }^{211}$. Furthermore, based on its crystal structure, Ndrg2 was proposed to be an enzymatically non-active member of a hydrolase family that is rather involved in molecular interactions than enzymatic activity. A role as a tumor suppressor was also observed in many other cancer types ${ }^{212}$. Next to its role in cell signaling, a nonsense mutation in Ndrg1 was described to be the source of a hereditary neuropathy of the peripheral nervous system ${ }^{213}$. mRNA levels of Ndrg2 are rising in parallel to neurite outgrowth during neuronal differentiation of PC12 cells and the protein localized to the plasma membrane of the cell soma and axonal growth cones. Overexpression of Ndrg2 did not change the number of neurites per cell, but they were significantly longer, which implicated a role in neurite outgrowth for Ndrg2 during neuronal differentiation ${ }^{214}$. No direct interaction or a functional connection of Ndrg with ion channels was described in the literature in the past. However, Ndrg was detected to associate with the membrane ${ }^{214}$, so from a spatial perspective the cross-links observed in this thesis are reasonable. In addition, both $\mathrm{Na}^{+} / \mathrm{K}^{+} \mathrm{ATPase}$ and PM CA belong to the family of structurally similar P-type ATPases ${ }^{109}$. It is therefore possible that the cross-links between these ion channels and Ndrg2 and 3 are an indication for a hitherto unknown interaction that might be functionally important.

\section{4-3-3 proteins and $\mathrm{Na}^{+} / \mathrm{K}^{+}$-transporting ATPase}

Similar to Ndrg, two members of the 14-3-3 adaptor protein family cross-linked to three isoforms of the alpha subunit of $\mathrm{Na}^{+} / \mathrm{K}^{+} \mathrm{ATPase}$. The 14-3-3 adaptor protein family consists of seven isoforms that form homo- and heterodimers ${ }^{215}$, which was also apparent from the identified cluster of 14-3-3 
proteins cross-linking with each other (figure 14). 14-3-3 proteins bind phosphorylated serine motifs in proteins and modulate their function ${ }^{215}$. Hundreds of interactors are known ranging from kinases, receptors, enzymes, and scaffold proteins to transcription factors ${ }^{215}$. Subsequent effects are thus multifaceted depending on the target proteins and include apoptosis, cell division, changes in metabolism, transcription and translation of effector proteins, cellular trafficking and cytoskeletal dynamics ${ }^{215}$. A general regulation of ion channel localization on the cell surface was also observed for several ion channels in different tissues and species ${ }^{216}$. Such a function was shown for 14-3-3 proteins in the case of $\mathrm{Na}^{+} / \mathrm{K}^{+}$ATPase by Efendiev and colleagues ${ }^{217}$. They have shown in coimmunoprecipitation experiments that 14-3-3 protein isoforms bind to $\mathrm{Na}^{+} / \mathrm{K}^{+} \mathrm{ATPase}$ depending on the phosphorylation state of serine 18 within the ATPase. Phosphorylation of the latter was identified to be crucial for clathrin-mediated endocytosis-dependent internalization of $\mathrm{Na}^{+} / \mathrm{K}^{+}$ATPase and reduction of its activity on the surface of renal tubule epithelial cells $s^{217}$. Phosphorylation of $\mathrm{Na}^{+} / \mathrm{K}^{+}$ ATPase leads to binding of a 14-3-3 protein to the phosphorylated serine, subsequent recruitment of phosphatidyl-inositol-3-phosphate kinase and generation of phosphatidyl-inositol-3-phosphate on the membrane. The latter increases the affinity of AP- 2 to $\mathrm{Na}^{+} / \mathrm{K}^{+}$ATPase serving as an adaptor for clathrin cage assembly and finally endocytosis $\mathrm{s}^{217}$. The same pathway of $\mathrm{Na}^{+} / \mathrm{K}^{+} \mathrm{ATPase}$ internalization might be important with regard to synaptic plasticity and excitability and the cross-links observed in this thesis are proof for an interaction of 14-3-3 protein with $\mathrm{Na}^{+} / \mathrm{K}^{+} \mathrm{ATPase}$ in brain synapses.

\section{G-proteins}

Subunits of the guanine nucleotide binding protein complex (G-protein) behaved promiscuously and cross-linked to many different ion channels like $\mathrm{Na}^{+} / \mathrm{K}^{+}$ATPase, ryanodine receptor, VDAC3 (figure 12figure 13), and V-type proton ATPase, as well as synaptic proteins like NSF and Stxbp1/Munc18-1 (figure 12). G-proteins are heterotrimeric complexes that associate with heptahelical receptors ${ }^{218}$. In response to a stimulus specific for each receptor, e.g. neurotransmitters, the G-protein coupled receptor (GPCR) catalyzes the exchange of GDP that is bound by the alpha subunit against GTP218. Subsequently, the alpha and beta/gamma components dissociate from each other and from the receptor and activate further effectors, for example downstream signaling cascades, enzymes, and ion channels ${ }^{218}$. An intrinsic GTPase activity of the alpha subunit leads to GTP hydrolysis and a restoration of the heterotrimeric complex, which ends its activity ${ }^{218}$. Ion channels can be regulated by G-proteins indirectly, for example an active G-protein can stimulate adenylate cyclase to generate CAMP, which in turn activates CAM P-dependent protein kinases to phosphorylate and modulate calcium channels in the heart. A direct regulation of channel gating would be by binding of an active G-protein subunit ${ }^{219}$. Such a regulation by direct interaction was not described in the literature for the proteins that crosslinked to G-protein subunits in this thesis. However, the identified cross-links indicate that the respective proteins are regulated by G-proteins or are involved GPCR signaling. NSF for example was shown to bind to the C-terminal tail of approximately a dozen G-protein coupled receptors and was proposed to be involved in receptor recycling after agonist stimulation, desensitization, and internalization of the receptor ${ }^{220}$. In addition, Stx1 but not Stxbp1/M unc18-1 co-purified with a Gprotein coupled receptor in a previous study ${ }^{221}$. Stx1 directly bound to G-protein beta/gamma complex, as well and might be involved in calcium channel modulation by G-protein signalling ${ }^{222}$. Since Stx1 and Stxbp1 are interacting at both resting and excited conditions in the synapse ${ }^{190}$, Stxbp1 nonetheless might be involved in these interactions, but was missed under the experimental conditions applied by Krasnoperov and colleagues ${ }^{221}$. Interestingly, no cross-links between G-proteins and metabotropic glutamate receptors were identified (figure 12). The latter are G-protein coupled receptors, which localize on the pre- or postsynaptic side, depending on the isoform ${ }^{223}$. However, 
during synaptosome preparation the post-synapse is often ruptured leaving only a postsynaptic density attached opposite of the active zone ${ }^{118}$. Thus, cytosolic G-proteins associated to metabotropic glutamate transporters on the postsynaptic side were possibly partially depleted before cross-linking.

Ras-related proteins

Two Ras-related proteins Rab3A and Rab10 were cross-linking to two proteins of the synaptic vesicle exocytosis machinery, i.e. Stxbp1/M unc18-1 and NSF (figure 12). Rabs are small GTP-binding proteins that are binding to synaptic vesicles in a GTP-bound form and dissociate from the vesicle upon GTPhydrolysis ${ }^{119}$. Rab3a, a member of the Rab family, is supposedly involved in synaptic vesicle recruitment from the reserve pool, as well as vesicle docking, priming and fusion ${ }^{224}$. No cross-links from Rab3a to its active zone interactors were identified, i.e. M unc13 and RIM ${ }^{83}$, which probably is due to their low abundance, as discussed above. Huang and colleagues ${ }^{224}$ have performed experiments that suggest a role for Stxbp1/Munc18-1 in Rab-GDP dissociation from the vesicle membrane, which is consistent with the cross-linking results ${ }^{224}$. NSF interacts with Rab6 in an ATP-dependent manner as shown by yeast two-hybrid system and co-immunoprecipitation ${ }^{225}$. Thus, an interaction with Rab10 as demonstrated by obtained cross-links might be possible, as well.

\section{Stxbp1/Munc18-1}

Stxbp1/M unc18-1 exhibited inter-protein cross-links to vacuolar protein sorting-associated protein 33B (Vp33B), tyrosine-protein phosphatase non-receptor type substrate 1 (Shps-1), and synaptojanin (figure 12). An interaction with Vp33B makes sense, since they both belong to the same family of Sec1/M unc18 proteins and interact with SNARE complexes ${ }^{226}$. Synaptojanin is a phosphatidyl inositol phosphatase that localizes with coated synaptic vesicles. It is involved in a late step of endocytosis where its enzymatic activity decreases the affinity of clathrin adaptors to the vesicle membrane and thus promotes clathrin coat disassembly. Synaptojanin mutants exhibit an impaired vesicle recycling ${ }^{95}$. Due to the function of synaptojanin and Stxbpl at the active zone and the surface of early endosomes, respectively, an interaction is spatially possible. Stxbpl was also shown to co-enrich with synaptic vesicles, which might be nonspecific but could enable an interaction between Stxbp1 and synaptojanin ${ }^{75}$. Shps-1 was initially identified in neuronal cells as a cell adhesion molecule that promotes neurite outgrowth in plated cells ${ }^{227}$. Domain analysis has shown the presence of immunoglobulin domains and tyrosine phosphorylation activation motifs indicating that Shps-1 is involved in cell interactions and signaling processes ${ }^{228}$. No functional connection to Stxbp1 was described in the literature so far. Thus, this interaction that is indicated by inter-protein cross-links might be an interesting target for follow-up experiments.

\section{Synapsin}

At last, two isoforms of synapsin exhibited cross-links to an uncharacterized protein, a cell adhesion molecule and a ubiquitin carboxyl C-terminal hydrolase (figure 12). Synapsins are phosphoproteins that reside on the membrane of synaptic vesicles and are thought to regulate the reserve pool of synaptic vesicles not bound to the active zone. They are binding to phospholipids, cytoskeletal proteins and oligomerize with themselves, thereby tethering synaptic vesicles to each other and maintaining the reserve poo $\mathrm{I}^{77}$. An oligomerization of synapsin isoforms was also observed in the cross-linking analysis. A contrary role for synapsins was described for inhibitory GABAergic synapses, where a synapsin knockout led to less synaptic vesicles, but the readily releasable pool at the active zone was more affected than the reserve poo ${ }^{229}$. The cross-link between synapsin 1 and neural cell adhesion molecule 1 might be due to the role of synapsin in synaptic vesicle tethering that could also involve 
cell adhesion molecules on the plasma membrane. The respective cross-link was located on the cytoplasmic side of the cell adhesion protein. Synapsin was identified with several ubiquitination sites in a proteomics study ${ }^{230}$, therefore, an interaction with a ubiquitinase as indicated by identified crosslinks might play a role in its regulation.

All of the inter-protein cross-links that indicate novel interactions discussed above are supported by multiple CSM s, but many are based on only one cross-linked residue pair. Inter-protein cross-links are frequently less abundant than intra-protein cross-links, because the respective residues within a protein are more consistently in close proximity to each other and the cross-link yield is therefore high. The distance between residues on two different proteins are on average more often subject to changes in case of e.g. transient interactions, thus the yield is low. Larger amounts of starting material and more extensive fractionation can aid to identify low-abundant inter-protein cross-links as discussed above. Nonetheless, the protein interactions identified in this cross-linking study are starting points for further experiments on these interactions and can be extended by complementary methods like coimmunoprecipitations, yeast two-hybrid system, immunofluorescence experiments, functional experiments in vivo and with purified proteins and others. However, validation of protein interactions by complementary methods might be difficult due to their respective inherent limitations. The great advantage of XL-MS is its ability to capture interacting proteins in their native environment at a specific time point under in vivo conditions. It furthermore allows the analysis of changing protein interactions within a system in response to a stimulus. Established methods for the detection of protein interactions often rely on the purification of proteins missing native posttranslational modifications, introduction of modifications to the protein, e.g. by adding a fusion-tag, and the usage of buffers that inadequately mimic cellular conditions. Furthermore, interactions are often examined without molecular crowding and the cellular context of the protein. Therefore, XL-MS is one of the most promising and unbiased methods to study synaptic protein interactions in their native environment

\subsection{Quantitative XL-MS applied on synaptosomes in resting and excited state}

XL-M S enables the identification of protein interactions in complex systems like synapses (chapter 4.3). However, biological functions of proteins are often mediated by transient protein interactions that change in response to stimuli, e.g. the protein machinery that enables the synaptic vesicle cycle in synapses. Synaptosomes are model systems for synapses that are biochemically active and excitable. Quantitative XL-MS analysis of synaptosomes under resting and excited conditions was therefore conducted to capture changing protein interactions and conformations in response to a stimulus. Nine cross-linking reaction replicates (corresponding to three biological replicates) of resting and excited synaptosomes were performed with isotopically labelled cross-linkers. Cross-linked residues were identified with the peptide-focused approach (identified cross-linked residues are discussed in chapter 4.3), and intensity ratios derived from the isotopic label were quantified. The abundance of numerous cross-linked residues significantly changed upon excitation, which indicated changes in protein interactions and conformations and is discussed in the following chapters 4.4.3 to 4.4.6. A majority of significantly changed cross-linked residues was observed in calcium-binding proteins (chapter 3.4.4.2) and ion channels (chapter 3.4.4.1), especially calcium channels. They were most prominently affected 
by a changed membrane potential, changed ion gradients across the membrane and the influx of calcium.

\subsubsection{General considerations regarding quantitative XL-MS of stimulated synaptosomes}

The stimulus applied in this study shifted synaptosomes in a permanently excited state. Cross-linking of stimulated synaptosomes for a prolonged time could have introduced artifacts, i.e. conformational changes that rather represented a seizure-like state than native exocytosis. Therefore, cross-linking for 30 to 60 min, as done by the Bruce lab ${ }^{60,61}$ and others was not feasible. Cross-linking of synaptosomes was performed for $10 \mathrm{~min}$ at $37^{\circ} \mathrm{C}$ instead, which resembled the glutamate release assay that was usually performed to monitor synaptosome excitability. Due to the increased cross-linker reactivity at higher temperatures, the cross-link yield was comparable to cross-linking for $1 \mathrm{~h}$ on ice according to SDS-PAGE (data not shown). Synaptosomes typically exhibited a saturation curve in the glutamate release assay with the bulk of glutamate being released within the first 2 min and barely any detectable release in the last $2 \mathrm{~min}$. Therefore, it is arguable whether $10 \mathrm{~min}$ cross-linking could already be too long to detect cross-links that are exclusive for the excited state. However, the saturation trend observed in the glutamate release assay is in part due to the enzymatic turnover of glutamate and NADPH generation, which is spectrophotometrically monitored. It eliminates glutamate from the cycle of transmitter release, reuptake by excitatory amino acid transporters and synaptic vesicle loading. During cross-linking of synaptosomes, no glutamate dehydrogenase is added. Thus, glutamate is not removed from the cycle, except for the glutamate that reacts with the cross-linker, and synaptic vesicle release is probably ongoing for a longer time period. In addition, it is hypothesized that the membranepermeable cross-linker diffuses into synaptosomes, shuts the metabolism down and freezes existing protein conformations quickly, followed by an accumulation of cross-links that further stabilize once frozen conformations. For example, once protein A and B are cross-linked together by one cross-linked residue, they are caught in close proximity, which facilitates further cross-linking events. It is therefore argued that cross-linking for 10 min captured protein conformations representative for the respective stimulation state, followed by the accumulation of representative cross-link species. The results presented in chapter 3.4.4 made sense in the context of synaptosome resting and excited state. Therefore, the presented approach was able to capture changing protein interactions and conformations that happened in a sub-second time frame in response to a change of membrane potential and calcium influx.

\subsubsection{Alternative approaches for quantitative XL-MS}

The Bruce $L a b^{60,61}$ also developed a quantitative approach for cross-linked complex samples. They utilized cells that were grown in SILAC medium containing either isotopically light or heavy $\left({ }^{13} \mathrm{C} /{ }^{15} \mathrm{~N}\right)$ lysine and arginine to differentially label proteins in the experimental and control condition. Subsequently, cells from both conditions were pooled, cross-linked, and analyzed jointly. Due to the differential label, they could quantify relative abundance ratios of the proteins as well as identified cross-linked peptides and residues deriving from the control and experimental condition ${ }^{60,61}$. The 
Bruce Lab employed this approach to analyze long-lasting interferences of physiological conditions, i.e. a chemoresistant HeLa cell line ${ }^{93}$ and the effect of an HSP90 inhibitor ${ }^{94}$.

In comparison to the Bruce lab, the present quantitative cross-linking approach to study conformational changes in synaptosomes in response to stimulation was novel in two aspects.

First, a chemical stimulus was investigated that had immediate effects on the conformation of e.g. calcium-binding proteins, as shown in chapter 3.4.4.2. Furthermore, the stimulation of synaptosomes triggered a complex sequence of multiply changing protein interactions. For example, a cis-SNARE complex is assembled in a sub-millisecond time frame, which is accompanied by vesicle fusion and followed by disassembly of the complex. Since the conformational changes were highly transient, prolonged cross-linking for an hour as done by the Bruce lab ${ }^{60,61}$ would not have been feasible due to the danger of introducing artifacts (see also discussion below).

Second, the cross-linker was used as a vehicle to introduce the isotopic label to a complex sample, instead of labelling the sample prior to the experiment. Therefore, the quantitative approach is much more versatile and can be applied to systems, for which introduction of a metabolic label is not readily available, e.g. synaptosomes. However, it lacks the information of changing protein abundances, which is helpful for the analysis of conditions that not only influence protein conformations but also protein expression. It should be noted that isotopically labelled cross-linkers were frequently used in the past for identification of cross-links in combination with the XQuest software ${ }^{40}$. Unlike this thesis, previous quantitative cross-linking efforts using isotopically labelled cross-linkers have focused on rather simple complexes compared to the synapse, for example chloroplast F-type ATPase ${ }^{55}$, bovine TRiC complex ${ }^{231}$, and complement protein $\mathrm{C} 3\left(\mathrm{H}_{2} \mathrm{O}\right)^{232}$.

An issue in the quantification approach at hand were missing values, i.e. cross-linked residues that were not persistently quantified in all replicates. As outlined in chapter 3.4.4, only 1,848 of overall 3,818 unique cross-linked residues (48\%) were quantified in at least three of nine cross-linking reaction replicates. Only 579 (15\%) unique cross-linked residues were identified in all nine replicates. Since cross-linked peptides are usually low abundant, they were especially prone to being missed in data-dependent LC-M Sacquisition. The issue with missing values was also the reason for nine analyzed replicates and a low cut-off filter of three valid values per cross-link prior to one-sample t-test.

Several strategies were developed in the past to overcome the missing value problem in proteomics quantification workflows. As mentioned in chapter 4.1.3, multiplex TMT labelling and quantification was recently established for cross-linked peptides ${ }^{59}$. Due to the isobaric TM T label, peptides originating from different conditions are selected together for MS2. Relative abundances can be quantified on MS2 level due to the cleavage of the mass reporter from the mass normalizer group within the TMT $\operatorname{tag}^{21}$. Therefore, an identified peptide is also quantified across almost all conditions and the share of missing values is very low.

Another approach is a label-free data-independent acquisition (DIA) analysis of cross-linked peptides, which was recently established ${ }^{58}$. However, $M$ üller and colleagues ${ }^{58}$ experienced increased coefficients of variation for label-free DIA quantified cross-linked peptides that were mixed with tryptic peptides originating from an E. coli lysate. They argued that a higher complexity of the sample led to interferences by co-eluting precursor and fragment ion masses ${ }^{58}$. Therefore, label-free DIA quantification is challenging for complex cross-linked samples like synaptosomes in terms of reproducibility.

The workflow applied here quantified cross-linked residues within and between the most abundant proteins. Quantitative results were dominated by intra-protein cross-links due to the on average lower abundance of inter-protein cross-links as discussed in chapter 4.3.3. Low signal intensity of cross-linked peptides is especially detrimental for quantification, since it leads to a low number of data points over 
the retention time, incomplete isotope envelopes, and a high signal to noise ratio ${ }^{54}$. Thus, quantification can suffer a high variability, which leads to fewer identified cross-linked residues that changed significantly.

Altogether, the quantitative analysis of changing protein interactions in synaptosomes as presented in this thesis was not attempted before. This thesis therefore analyzed the most complex and transiently changing sample by quantitative XL-MS, to date.

\subsubsection{Proteins residing in intracellular $\mathrm{Ca}^{2+}$-storage organelles}

Significantly changed cross-linked residues were observed for proteins residing in the endoplasmic reticulum (ER) and mitochondria. These intracellular organelles are involved in $\mathrm{Ca}^{2+}$-storage and are also present in the pre- and postsynapse ${ }^{69-71}$. This implies that proteins residing in these intracellular compartments also experienced consequential effects of membrane depolarization. Calcium influx occurs locally through voltage dependent calcium channels at the active zone. Ions diffuse 10 to 100 $\mathrm{nm}$ into the synapse where they are sequestered by calcium-binding proteins and are quickly cleared from the synapse by ion channels ${ }^{233}$. The bulk of calcium is cleared by potassium-dependent and independent sodium/calcium exchangers and plasma membrane $\mathrm{Ca}^{2+}$-transporting ATPase (PM CA) on the surface of the plasma membrane ${ }^{234}$. Although the ER and mitochondria are important intracellular calcium stores, the latter seem to only play a role if the calcium load is large, for example after a prolonged sequence of action potentials ${ }^{234,235}$. In addition, higher transient calcium concentrations were observed after stimulation in mitochondria-free synapses in comparison to synapses containing mitochondria ${ }^{113}$. Since the chemical stimulation applied in this study sent synaptosomes in a permanently excited state, high calcium concentrations were probably present in the synapse, thereby leading to a mitochondrial participation in calcium clearance and elevated calcium concentrations in the mitochondrial matrix. This could be a reason for the significantly changed cross-links observed in mitochondrial proteins. A regulatory effect of intramitochondrial calcium on ATP synthesis and transport was described for cardiomyocytes ${ }^{236}$, including a stimulatory effect on ATP synthase ${ }^{237}$. A similar effect would make sense for synaptosomal mitochondria with regard to the increased ATPdemand of ion transporting ATPases to restore the ion gradient across the membrane. However, only few intra-protein cross-links were significantly changed in each VDAC, creatine kinase, ADP/ATP translocase, succinate dehydrogenase and ATP synthase, which does not allow conclusions on a structural level. The lack of additional significantly changed cross-linked residues was probably a consequence of the successful depletion efforts described in chapter 3.4.1.2. Since mitochondria exhibited significant changes in response to stimulation of synaptosomes, they could be examined in a follow-up study. Synaptosomes could be processed and stimulated along the lines of this study with a focus on mitochondrial proteins by biochemical enrichment, e.g. as observed for the pellet fraction below the $1.2 \mathrm{M}$ sucrose phase in figure 7.

In contrast to mitochondria, the ER seems to be negligible in calcium clearance. It was shown that the sarcoendoplasmic reticulum $\mathrm{Ca}^{2+}$-transporting ATPase (SERCA) on the surface of the ER is not contributing to presynaptic calcium clearance ${ }^{234}$. However, significantly changed cross-linked residues were identified for SERCA and were located in the head group of the ATPase, which exhibits the greatest conformational changes during an ion translocation cycle (figure 16). Naturally, SERCA is active at both resting and excited conditions and adopts all conformational E1 and E2 states of the 
translocation cycle $\mathrm{e}^{112}$. The difference between the two states probably is which conformations are predominating the cycle due to e.g. lack of calcium in the cytoplasm. The latter condition was present in synaptosomes during resting conditions. Accordingly, the one cross-link that was high abundant at resting condition had a $\mathrm{C} \alpha$ - $\mathrm{C} \alpha$ distance of $56.1 \AA$ (K205-K492, blue line in figure 16B) and is overlength in the E1 state of SERCA that binds $2 \mathrm{Ca}^{2+}$ ions. This cross-link implied that SERCA was predominantly in the $\mathrm{E} 2$ state under resting conditions, i.e. after calcium translocation, because this was the only state where lysine C $\alpha$ atoms were within the physically possible distance of the DSS spacer plus two lysine side chains. Similarly, one cross-link between K352 and K492 was enriched at excited conditions and had the shortest $\mathrm{C} \alpha$ - $\mathrm{C} \alpha$ atom distance in the E1-P state (16.3 $\AA$, figure 16C). Therefore, SERCA was predominantly in the E1 state at excited conditions and was presumably binding calcium. This observation indicated that the large rotation of the actuator domain from E1 to E2 state ${ }^{112}$ could be a bottleneck that takes a relatively long time, thus leading to the observed enriched cross-link. Large movements also occur in the transmembrane helices of SERCA during the translocation cycle, which was not detected in this thesis. However, hydrophobic transmembrane domains usually contain few charged residues like lysine. Tryptic and cross-linked peptides in particular have therefore unfavorable properties for detection by LC-MS. Altogether, the observed cross-links that changed their abundance implicate domain movements that agree with an ion pump that was actively transporting calcium ions from the cytoplasm to the lumen of the ER.

Apart from SERCA, several cross-linked residues with a significantly changed abundance were identified in inositol-1,4,5-trisphosphate receptor (I3PR). I3PR is also a calcium channel in the ER, but is regulated by binding of its ligand inositol-1,4,5-trisphosphate. Accordingly, several less abundant cross-linked residues at excited conditions were identified near the ligand binding pocket (figure 19). However, the conformational changes that are accompanied by ligand binding and channel opening are not large enough to explain the decreased cross-link abundance, because the residues are in crosslinking distance in both conformations (figure 19). The cross-links could be less abundant due to a sterical hindrance by a large domain movement indicated by the two overlength cross-links not explained by the closed and open models (red lines, figure 19). Since cytoplasmic calcium levels are high during stimulation, it is anticipated that I3PR would adopt a closed or inhibited state to prevent a further increase in calcium levels. In addition to a closed and open state, patch-clamp recordings of single I3PR by lonescu and colleagues ${ }^{238}$ have indicated that the channel could adopt a third inactive or inhibited state that turned it nonresponsive to both ligand and cargo. This state was reversible by wash-out of the ligand from the bath solution. Eventually, every I3PR became inactive during patch clamp recordings, but inactivation was accelerated by an order of magnitude at low inositol-1,4,5triphosphate- and high calcium concentrations ${ }^{238}$. Cytosolic calcium concentrations are probably high during excitation and no further perturbations were applied to the sample that could raise the inositol1,4,5-triphosphate concentration. Therefore, the high abundant cross-links under excited conditions observed in this thesis could be associated with such an inactive or inhibited I3PR mediated by a large domain movement not described by cryo-EM or other structural biology methods, so far.

At last, the two intralumenal ER proteins calreticulin and ERp29 also exhibited significantly changed cross-links. The former is a calcium-binding chaperone that is involved in folding of glycoproteins in the endoplasmic reticulum. Calreticulin is considered as a major ion storage protein binding up to $50 \%$ of calcium in the endoplasmic reticulum ${ }^{162}$. A cross-link from lysine residue 164 to 351 was identified with a significantly higher abundance in an excited state indicating a conformational change. This observation was in agreement with previous studies, in which calreticulin was trypsin-sensitive at low calcium concentrations but was partly protected from complete digestion in presence of calcium ions. 
A $27 \mathrm{kDa}$ N-terminal fragment that covers lysine 164 of the quantified cross-link was resistant to digestion even after prolonged incubation with trypsin ${ }^{239}$.

ERp29 on the other hand does not have calcium binding properties in vitro ${ }^{163}$, but exhibited a significantly less abundant cross-link during excitation of synaptosomes. ERp29 belongs to a family of protein disulphide isomerase-like chaperones, which includes several members known to bind to calreticulin and assist in protein folding. Although calreticulin co-eluted during ERp29 purification, ERp29 did not seem to aid calreticulin in its chaperone function ${ }^{163}$. Nonetheless, ERp29 was shown to bind calreticulin in a 1:1 stoichiometry ${ }^{240}$ and is present as a homodimer in solution ${ }^{163}$. Since it is not binding calcium, the significant change in cross-linking might be the effect of an altered binding behaviour to e.g. calreticulin.

As discussed above, Kim and colleagues ${ }^{234}$ have measured the contribution of SERCA and in extension the ER to calcium clearance in synapses and deduced it to be negligible ${ }^{234}$. However, significantly changed cross-links indicate that SERCA was active, I3PR possibly exhibited an inactive conformation and calcium-binding calreticulin experienced a conformational change in response to stimulation. These observations imply that cyto plasmic calcium was pumped into the ER. In light of the observations of Kim and colleagues ${ }^{234}$, this might be due to the tetanic chemical stimulation applied in this thesis and therefore partially artificial. Because of permantly increased membrane potential, higher calcium levels were present in the cytoplasm than during naturally occurring depolarization events, thereby leading to calcium uptake by the ER. On the other hand, Kim and colleagues ${ }^{234}$ have performed their assays on the calyx of Held ${ }^{234}$, a giant synapse in the auditory system with unique morphological features that facilitate electrophysiological recordings ${ }^{241}$. It cannot be excluded that the ER might participate in $\mathrm{Ca}^{2+}$-clearance in other types of synapses.

\subsubsection{Plasma membrane ion channels}

The P-type $\mathrm{Na}^{+} / \mathrm{K}^{+}$ATPase and plasma membrane $\mathrm{Ca}^{2+}$-transporting ATPase (PMCA) both exhibited significantly changed cross-linked residues that were similarly distributed in the head group as quantified for SERCA (chapter 4.4.3). Unfortunately, no crystal structures exist for all possible conformations of these two ATPases, but major domain movements will probably resemble those reported for SERCA. Accordingly, PMCA also exhibited significantly changed cross-links that suggest the E1 state, presumably phosphorylated and binding two calcium ions, to be predominant in response to stimulation (figure 17). Cross-links accumulated in a state of the translocation cycle right before a major conformational movement that enables the release of calcium to the extracellular side. Unlike SERCA, PMCA also exhibited cross-links to unresolved loops of the cryo-EM structure, which are associated with regulatory functions. The unresolved C-terminus contains an auto-inhibitory domain that binds to acidic phospholipids and calcium/calmodulin ${ }^{146,147}$. The three significantly less abundant cross-links to this domain indicated that the flexible C-terminus was further away from the body of PMCA under excited and high calcium conditions, thereby not inhibiting the activity of the pump. Disinhibition is mediated by calcium/calmodulin binding ${ }^{96}$, but no cross-links between PM CA and calmodulin were identified. The unresolved loop shown as a black dashed line in figure 17 also binds acidic phospholipids, which enhances PM CA activity synergistically with the C-terminal domain ${ }^{96}$. The significantly changed cross-links suggest that conformational changes occurred in these domains, 
which possibly shifted PMCA in a more active state and enabled a faster clearance of calcium from the cytoplasm.

Contrary to PM CA and SERCA, the $\mathrm{Na}^{+} / \mathrm{K}^{+}$ATPase did not exhibit significantly changed cross-links that agreed with exclusively one preferred state under excited conditions. As already mentioned in chapter 3.4.4.1, the ion pump is an antiporter that experienced high concentrations of cargo on both sides, i.e. high potassium concentrations in the extracellular space and high sodium concentrations in the cytosol. Therefore, significantly changed cross-links fit to both the E1 state as well as the E2 state. Interestingly, one inter-protein cross-link to the same lysine residue between two isoforms was significantly more abundant under excited conditions (K202-K212, figure 18C). Assuming that the alpha subunit dimerized as observed in the crystal lattice reported by Kanai and colleagues ${ }^{115}$ (figure $18 \mathrm{C}$ ), two other cross-linked residues that were higher abundant upon stimulation were possibly interprotein cross-links (K202-K487, K212-K477, figure $18 \mathrm{C}$ ). $\mathrm{Na}^{+} / \mathrm{K}^{+}$ATPase was reported to assemble to dimers and even tetramers ${ }^{149}$. Therefore, other cross-linked residues interpreted as intra-protein cross-links could be in fact inter-protein cross-links. $\mathrm{Na}^{+} / \mathrm{K}^{+}$ATPase is more active under excited conditions, because cargo is binding to the pump almost instantly after each translocation step, in contrast to resting conditions defined by low levels of potassium and sodium on the extracellular and cytoplasmic side, respectively. Significantly higher abundant inter-links under excited conditions could therefore be a result of cooperativity, i.e. dimer interactions could facilitate ion translocation or make it more efficient.

PMCA associates with basigin or neuroplastin ${ }^{146}$, whereas the catalytically active alpha subunit of $\mathrm{Na}^{+} / \mathrm{K}^{+}$ATPase oligomerizes with a beta and gamma subunit ${ }^{149}$. No significantly changed cross-links were identified between catalytically active and accessory subunits, although the latter have regulatory functions for ion transport ${ }^{146,242}$ or are supposedly involved in the formation of oligomers of $\mathrm{Na}^{+} / \mathrm{K}^{+}$ATPase holoenzymes ${ }^{149}$.

\subsubsection{Calcium and calmodulin-binding proteins}

Anxa6 exhibited a significantly changed cross-link in each of its two perpendicular lobes that are virtually the same cross-link (figure 23). Upon calcium-binding, Anxa6 undergoes a conformational change to a co-planar orientation of the two lobes, binds to the plasma membrane and presumably functions as an ion channel ${ }^{159,}{ }^{160}$. A less abundant cross-link upon calcium-binding in lobe $B$ could be explained by sterical hindrance by the bound ion or plasma membrane, but the same should be the case for lobe A, which exhibited a more abundant cross-link (figure 23). The two cross-links rather indicated that the annexin repeat domains move relative to each other in response to stimulation. Such a movement of annexin repeat domains was predicted for a calcium- and membrane-bound annexin $\mathrm{V}$ in a previous study ${ }^{161}$, because the protein is strongly dipolar in terms of electrostatic potential. An electrical field across the membrane and an asymmetric charge distribution would therefore lead to differentially acting forces on annexin repeat domains after plasma membrane binding ${ }^{161}$. If the lobes of Anxa6 had similar properties as Annexiv V, it could explain the contrary quantified cross-link in the two lobes as a result of relative movement of annexin repeat domains to each other. Benz et al. ${ }^{160}$ performed patch clamp ion channel recordings of membrane- and calciumbound wildtype Anxa6, in addition to lobe $A$ and lobe $B$ in isolation. Lobe $B$ displayed stronger ion 
currents than lobe $A$, which is possibly in accordance with the significantly decreasing cross-link observed in this study and a somewhat more open ion channel in lobe $B^{160}$.

Syt1 was identified with several inter-links to other proteins involved in exocytosis, but no significantly changed inter-links were quantified apart from two less abundant intra-links upon stimulation (figure 22). However, these two cross-links indicated that the $\mathrm{Ca}^{2+}$-sensor Syt $1^{243}$ was responding to elevated calcium levels. One cross-link in the C2B domain cross-linked to a loop that coordinates calcium ions ${ }^{90}$ and was possibly less abundant due to sterical hindrance upon calcium binding or accompanying conformation changes. The other cross-link in the C2A domain was significantly less abundant with a fold-change below 1.5 but cross-linked neighboring lysine residues, which are limited in conformational flexibility that can explain significantly changed cross-links. It was shown before that the Syt1 C2A domain is involved in calcium-mediated phospholipid binding ${ }^{79}$. Negatively charged amino acid residues in the C2A domain loops bind positively charged calcium ions electrostatically, which again bind to negatively charged phospholipid head groups. The calcium binding sites are furthermore enclosed by positively charged amino acid residues that contribute to phospholipid binding ${ }^{79}$. Although the lysine stretch from 189 to 192 involved in the decreasing cross-link was not examined in the study of Zhang et al. ${ }^{79}$, it could also be involved in phospholipid binding in presence of calcium. The cross-link was possibly decreasing due to sterical obstruction by the plasma or synaptic vesicle membrane, irrespective whether or not these lysine residues are directly contributing to binding.

A significantly less abundant cross-linked residue was observed in the catalytically inactive kinase domain of calcium/calmodulin-kinase-like vesicle-associated protein (Camkv) ${ }^{153}$ (chapter 3.4.4.2). Since the changing cross-link was not observed in the calmodulin binding domain, which is located Cterminal of the kinase domain, it could be a consequence of an induced conformational change instead of e.g. sterical hindrance by bound calcium/calmodulin. The precise mechanism of Camkv function is still not clear. Several somatic mutations were identified in the Camkv gene in different cancer genomes ${ }^{244}$. Expression of Camkv in dendrites was increased upon local synaptic activity and overexpression led to an increased number of dendrites. This effect was independent of a possibly undetected kinase activity, but reportedly depends on calmodulin binding ${ }^{154}$. The obtained data on Camkv in this thesis was too scarce to draw conclusions on its function. However, Camkv seemed to undergo a conformational change upon calcium influx and probably calmodulin binding, which might mediate downstream signaling ${ }^{154}$.

Two significantly less abundant intra-links within Camkll indicated that the enzyme was enzymatically active in response to stimulation (figure 20). One cross-link between the hub and kinase domain indicated that the kinase was extended and not in an auto-inhibited state ${ }^{151}$. Another cross-link involving lysine 43 was probably less abundant due to sterical hindrance by ATP binding to the residue or a conformational change induced by ATP binding ${ }^{152}$. These cross-links therefore agree with the notion that Camkll is activated by calcium and calmodulin binding. Its activity is important for synaptic plasticity, learning and memory ${ }^{102}$. However, Camkll A was also described to regulate synaptic vesicle docking independent of its kinase activity ${ }^{245}$. Camkll also displayed compelling inter-protein cross-links to a neutral ceramidase. Three cross-links clustered to the hub domain of two Camkll isoforms and one cross-link was two-fold more abundant indicating an association of both proteins upon stimulation. Neutral ceramidase is an important enzyme in sphingolipid metabolism, whose activity also generates the second messenger sphingosine. The enzyme was reported to be glycosylated, which mediates an association with the plasma membrane ${ }^{246}$. The interaction with the enzyme might enable Camkll to localize closer to phosphorylation targets in the synapse during stimulation. Neutral ceramidase is also important for the regulation of synaptic vesicle exocytosis. M utation of a neutral ceramidase homolog 
in Drosophila melanogaster led to a reduced synaptic vesicle cycling and reserve pool and impaired synaptic vesicle release ${ }^{247}$. These effects are probably due to its role in sphingolipid metabolism and regulation of lipid rafts, which mediate protein trafficking and aggregation of proteins to membrane complexes $^{248}$. Camkll and neutral ceramidase both fulfil important roles in synaptic transmission, but an interaction was not described in the past. Thus, it is an interesting target for a follow-up analysis, whether this interaction has a functional relevance.

\subsubsection{Cytoskeleton and proteins that do not bind calcium}

Two integral membrane spanning proteins that are not known to directly bind calcium have changed in their dimerization behavior in response to excitation. Cend1 seemed to monomerize, because all significantly changed intra-protein cross-links were less abundant upon stimulation. However, no dimer cross-links to the same residue and peptide were identified. Since Cend1 itself is not known to bind calcium, the mechanism of monomerization remains ambiguous and could involve other calciumsensing proteins. Cend1 could also bind to another protein, thereby masking the protein surface and preventing the formation of the significantly changed cross-links. Cend1 is expressed on the surface of mitochondria and the ER, two organelles with a role in calcium storage ${ }^{166}$, and was also identified in the docked synaptic vesicle fraction by Boyken and colleagues ${ }^{130}$. Its overexpression led to a decreased release of calcium from intracellular stores ${ }^{165}$. The findings of this thesis could be a result of Cend1 regulatory mechanism on calcium release. Therefore, it might be an interesting target for follow-up experiments.

ADAM 23 on the other hand appeared to dimerize upon excitation, because one dimer-link to the same residue and peptide was significantly more abundant. So far, the protein was described as a negative regulator of integrin-mediated cell proliferation and the silencing of its gene is often observed in different cancer types ${ }^{167}$. A direct connection to calcium signaling is not apparent, but ADAM 23 is also binding to integrins, which modulate ligand binding and affinity via divalent cations like calcium ${ }^{167}$. The dimerization could therefore be a result of an altered binding mode to integrin molecules on neighbouring cells.

Several cytoskeletal proteins exhibited significant changes indicating a regulation by calcium. First and foremost, Spectrin alpha and beta and ankyrin, had more abundant inter-protein cross-links between each other. Spectrin alpha is also binding calcium ions ${ }^{156}$, but it is discussed here and not in chapter 4.4.5, because other cytoskeleton proteins also exhibited more abundant inter-links, i.e. between alpha- and beta-tubulin, alpha- and beta-centractin, and actin and spectrin beta. A possible explanation could be a calcium-driven regulation of the cytoskeleton system. Actin and microtubules are the main components of morphological plasticity for axon growth and guidance, dendritic branch dynamics, and dendritic spine morphogenesis ${ }^{249}$. Cytoskeleton reorganization is not directly regulated by calcium influx but is a result of a cell signaling cascade. In the post-synapse for example, NMDA receptor activation by binding of glutamate leads to calcium influx and activation of Camkll. Downstream of CamkII, Cdc42 and Rac, two small GTPases of the Rho family, are activated by guanine exchange factors and in return activate nucleation promoting factors, which leads to actin reorganization and actin-filament branching via Arp2/3 complexes $^{106,250}$. M urakoshi and colleagues ${ }^{106}$ have shown that the dendrite morphology changed within seconds upon activation of this cascade and 
that changes persisted for more than $30 \mathrm{~min}^{106}$, which is well in the time frame of cross-linking performed in this thesis.

Similarly, microtubules enter and polymerize into dendritic spines in a targeted manner when the latter receive synaptic calcium signals. M icrotubule entry is also depending on filamentous actin and drebrin, which connects microtubules and filamentous actin ${ }^{107}$. Similar signaling cascades regulating the cytoskeleton are also present in the presynapse ${ }^{103}$.

Alpha and beta centractin are cytoskeleton proteins with 50 to $70 \%$ sequence homology to actin. They mostly localize to the centrosome in the perinuclear region ${ }^{251}$. When overexpressed, centractin also formed filaments and was shown to interact with spectrin within the dynactin complex. Centractin was proposed to enable the dynein-mediated transport of organelles and vesicles along microtubules by binding to spectrin on the surface of the membrane ${ }^{252}$. Increased interactions between alpha and beta centractin, as indicated by more abundant cross-links, could be a result of altered transport dynamics in response to stimulation.

Calcium/calmodulin binding to spectrin alpha was shown to render the protein and an adjacent spectrin beta molecule in the spectrin heterotetramer susceptible to calpain cleavage. Calpain is a calcium-dependent cysteine protease, which is activated by NMDA receptor activation. Spectrin cleavage by calpain is associated with synaptic and neuronal plasticity ${ }^{108}$.

At last, three chaperones exhibited significantly more abundant cross-links, one intra-link within heat shock protein 90 alpha, and two inter-links from heat shock cognate $71 \mathrm{kDa}$ protein (Hsc70) to heat shock protein $105 \mathrm{kDa}$, and mitochondrial stress-70 protein. As mentioned above, ER chaperones ERp29 and calreticulin also displayed significantly changed intra-links indicating a general pattern of conformational changes in chaperones upon stimulation. $\mathrm{Hsc70}$ is involved in clathrin cage disassembly with the help of auxilin ${ }^{96}$. However, no inter-links were identified or quantified that indicated increased interactions between the two proteins in response to excitation and increased synaptic vesicle fusion and clathrin-mediated endocytosis events. Instead, the inter-link between Hsc70 and heat shock protein $105 \mathrm{kDa}$ suggested a less active chaperone. The latter was reported to inhibit the ATPase activity of $\mathrm{Hsc70}$, thereby negatively regulating the $\mathrm{Hsc70}$ chaperone system ${ }^{253}$.

\subsubsection{Synaptic protein complexes that did not display conformational changes upon excitation}

Cross-linked residues were not identified or quantified for many proteins and protein complexes, whose behavior upon synaptic stimulation is well-known. Unfortunately, no conclusions on missing interactions can be made based on missing cross-links. Proteins might have been low abundant and were not detected by LC-M S. Specific cross-links could have formed during the cross-linking reaction, but the cross-linked peptides were not selected for fragmentation or had a poor fragmentation pattern during LC-MS analysis. In contrast, three protein complexes were identified with several inter- and intra-protein cross-links that did not change significantly, i.e. voltage-dependent calcium channel, adaptor protein AP-2 complex, and Stx1 bound by Stxbp1/M unc18-1 (chapter 3.4.4.5, figure 26). A possible reason could be that conformational changes are too transient to be detected by XL-M S with an NHS-based cross-linker. Other cross-linker chemistries, e.g. diazirine cross-linkers, could be more successful due to faster cross-linking kinetics and inducibility by UV light, respectively ${ }^{254}$. In addition, reactive residues could be missing on domains that are changing their conformation in response to 
stimulation. The developed workflow could be unsuitable for detecting particular significant changes due to some steps in the procedure that might lead to a loss in transient protein interactions. Those steps might include, e.g. the duration of cross-linking, or the continuous sucrose density gradient centrifugation. The osmotic shock by the high sucrose concentration in the gradient might be an additional stress factor for synaptosome viability.

Furthermore, only a subset of docked and primed synaptic vesicles react to calcium influx at the active zone during excitation. The reserve and cycling pool is not responsive but available for subsequent action potentials. Therefore, cross-linking of stimulated synaptosomes might capture the majority of synaptic proteins in a conformation similar to the resting state. At last, the variability between biological and reaction replicates might have prevented the detection subtle changes in cross-linking abundance, e.g. due to varying synaptosome preparations. 


\subsection{Summary and conclusions}

The present thesis enabled the identification of an extensive protein interaction network covering many known interactions, in addition to novel interactions. Examples were cross-links between pyruvate kinase and two ion transporting ATPases, synapsin and a ubiquitinase, the interconnetivity of Ndrg, 14-3-3 scaffold proteins and G-proteins to different ion channels, as well as interactions observed for Stxbp1/Munc18-1. Furthermore, abundance changes of cross-linked residues and thereby changing protein interactions as well as protein conformational changes were quantified in response to synaptosome stimulation. Many significantly changed cross-linked residues were observed in $\mathrm{Ca}^{2+}$ - and $\mathrm{Ca}^{2+} /$ calmodulin-binding proteins, e.g. synaptotagmin, Anxa6, alpha spectrin, and Camkv. Ion channels in the plasma membrane (PMCA, $\mathrm{Na}^{+} / \mathrm{K}^{+} \mathrm{ATPase}$ ) and in the membrane of the ER (SERCA, I3PR) and mitochondria (VDAC) exhibited significantly changed cross-linked residues under excited conditions. The implied conformational changes agreed with the respective ion channel function and reported behaviour in response to a changing membrane potential, altered ion gradients and $\mathrm{Ca}^{2+}$ influx. Moreover, previously unknown conformational changes were observed in this thesis, e.g. a major domain movement in I3PR that might turn the channel inactive, a possible monomerization of Cend1, and an enhanced interaction between Camkll and neutral ceramidase.

The aforementioned results were facilitated by the development of three novel strategies for the analysis of synaptosomes. Two biochemical approaches were established for synaptosome preparation to deplete co-migrating proteins originating from myelin fragments and intrasynaptosomal mitochondria. These depletion steps enabled a stronger focus on cross-linked residues within synaptic proteins. In addition, a peptide-focused database search approach was developed for the analysis of cross-linked residues in complex samples like synaptosomes. The approach circumvented the problem of an exponentially increasing database search space in the XLMS analysis of complex samples. Peptides that evidently participate in the cross-linking reaction were identified and used for database search, instead of considering all theoretically cross-linkable peptides derived from a proteome. The approach was benchmarked on purified cross-linked complexes, as well as on in vivo cross-linked bacteria and was shown to be suitable for XL-M S applications. The focus on peptides reduced the database search search space of cross-linked synaptosomal samples and enabled their analysis by XL-MS in a fraction of time and with a higher sensitivity.

$M$ any more significantly changed cross-linked residues were observed in synaptosomes covering diverse functions like the cytoskeleton, energy metabolism and cell adhesion. However, the output of significantly changed cross-linked residues per protein was often too low for a confident interpretation of its meaning. In addition, only few significantly changed inter-protein cross-links were identified. This thesis was only able to capture the most abundant and most profound changes in protein interactions and conformations in response to stimulation. Some reasons for the limited scope were probably due to the limitations of XL-MS, e.g. cross-linker chemistry and kinetics, an unfavorable distribution of reactive residues on proteins, sensitivity limitations of mass spectrometry and others.

However, XL-MS offers an unprecedented possibility for the proteome-wide analysis of protein structure and interaction dynamics in vivo in contrast to other structural methods like $X$-ray crystallography and cryo-EM. The method is still in its infancy and will probably further improve in its application scope, analytical possibilities, and depth of obtained information. Furthermore, XL-M S will benefit from continuously improving MS instrument sensitivity and the development of better algorithms in the future. 


\subsection{Outlook}

With regard to the XL-MS analysis of synaptosomes, a more exhaustive fractionation by SCX chromatography in combination with pSEC could help to identify more cross-linked residues and interprotein cross-links. In addition, adoption of a quantification protocol based on TM T-labelling could help reduce the dominance of missing values that impaired the quantification within this thesis.

Several novel protein interactions and conformational changes in response to stimulation were identified and quantified. As discussed in chapter 4.3.3, these observations can be further pursued and verified by complementary methods, which was not the scope of this thesis.

At last, significant conformational changes were apparently present in mitochondrial proteins but were not comprehensive due to the efforts to deplete mitochondrial proteins. A follow-up study based on workflows established here could focus on the influence of chemical stimulation on intrasynaptosomal mitochondria. 


\section{Appendix}

\subsection{Supplementary figures}
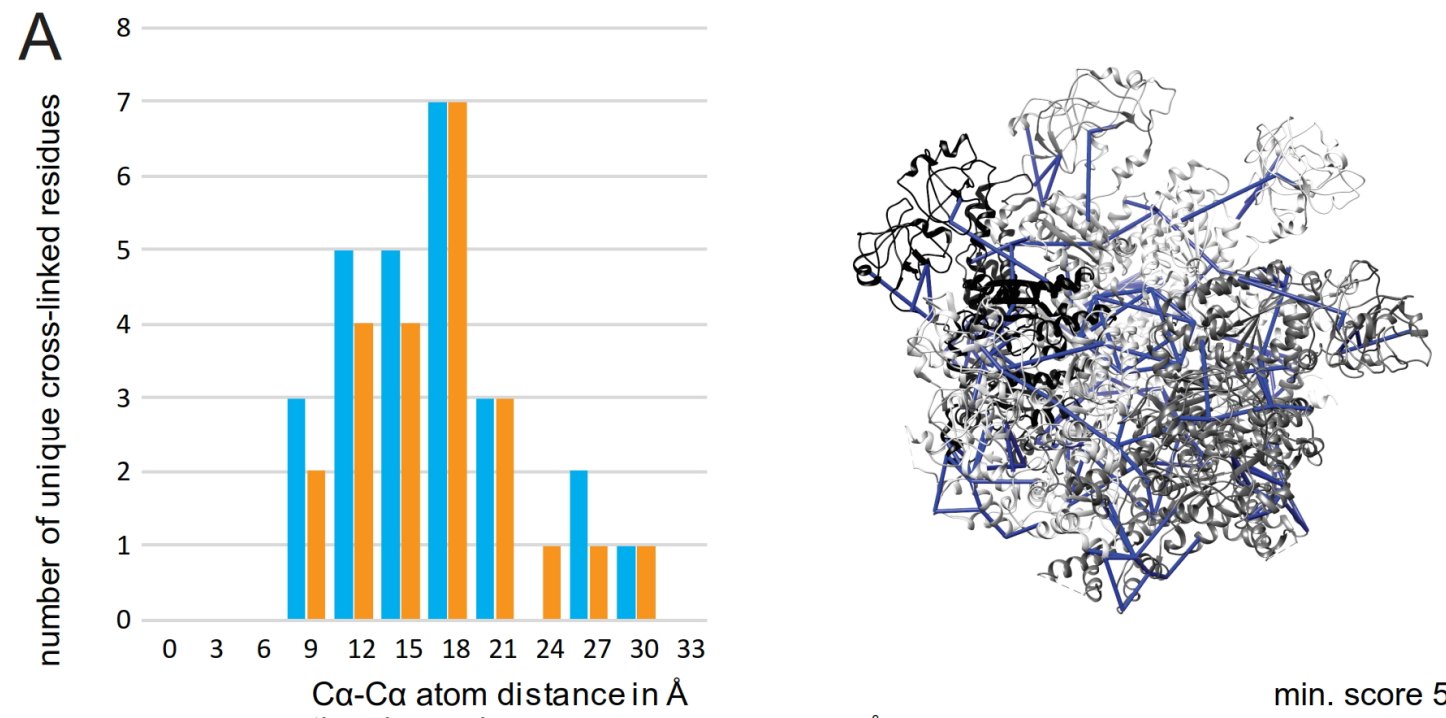

conventional search

(26 links, $0.0 \%>30 \AA)$

peptide-focused search (23 links, $0.0 \%>30 \AA$ )

$\min$. score 5

B

30

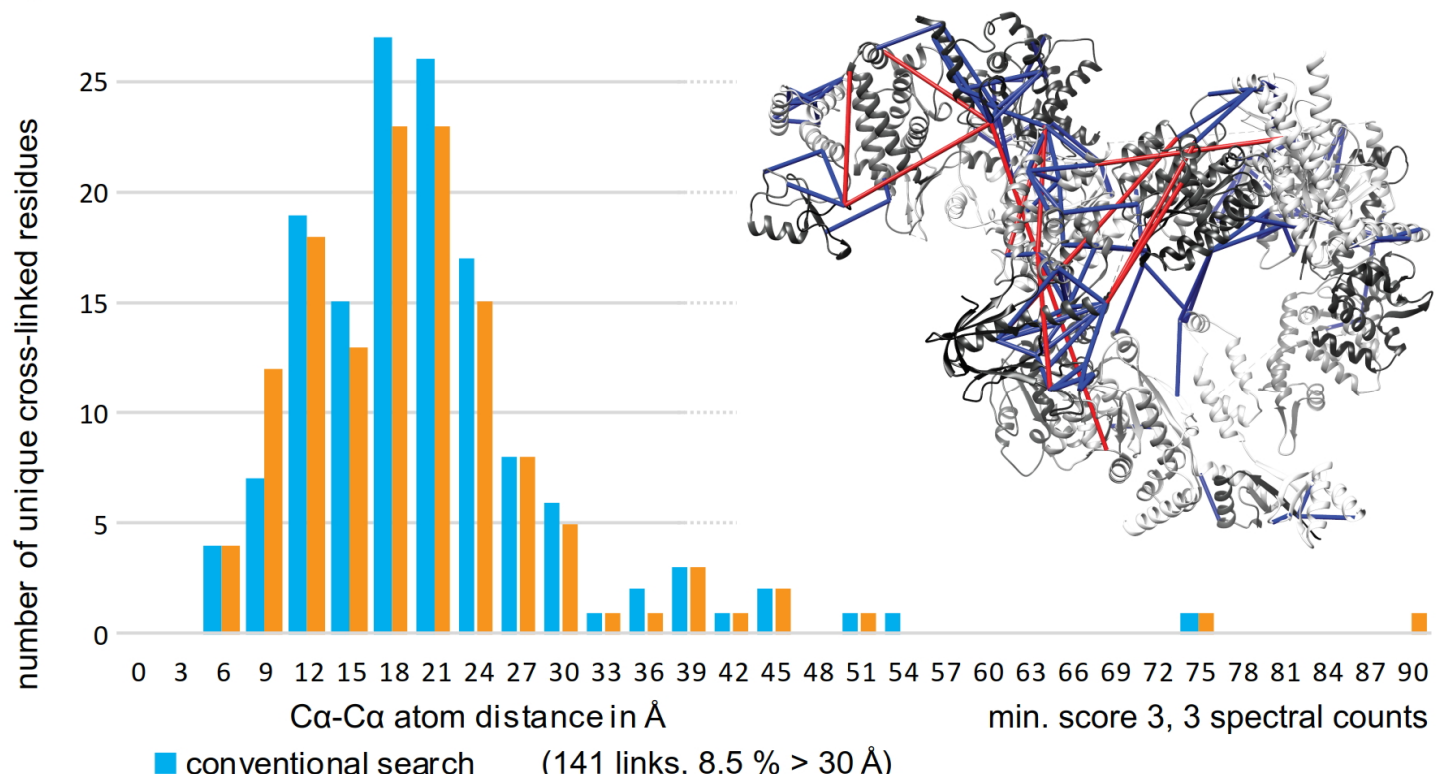

conventional search (141 links, $8.5 \%>30 \AA)$

peptide-focused search (132 links, $8.3 \%>30 \AA$ )

Supplemental figure 1: Comparison of conventional cross-linking with the peptide-focused approach on purified proteins. Transitional endoplasmic reticulum ATPase (p97, A, pdb: 5FTN) ${ }^{138}$, and transcription factor IIH (TFIIH, B, pdb: 50QM ) ${ }^{125}$ were processed as outlined in chapter 3.1 and resulting cross-links were filtered for a minimum pLink score of 5 (p97) or 3 and at least 3 identified spectra (TFIIH). C $\alpha$-C $\alpha$ atom distances between linked lysines are plotted as histogram and are exemplary shown on the respective crystal structure (grey to black) for the peptide-focused approach. Links greater than $30 \AA$ are considered overlength and are depicted in red. 


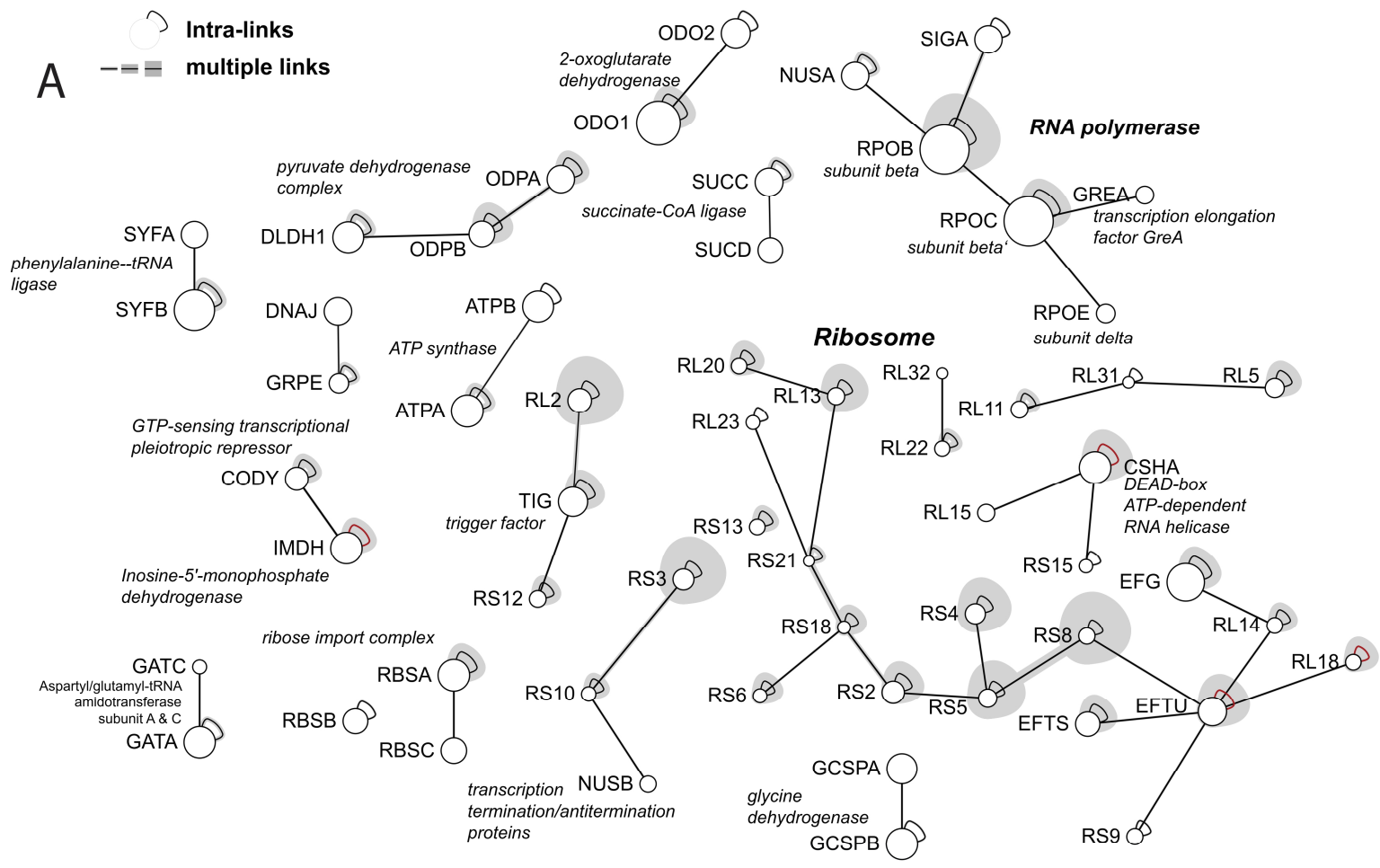

B

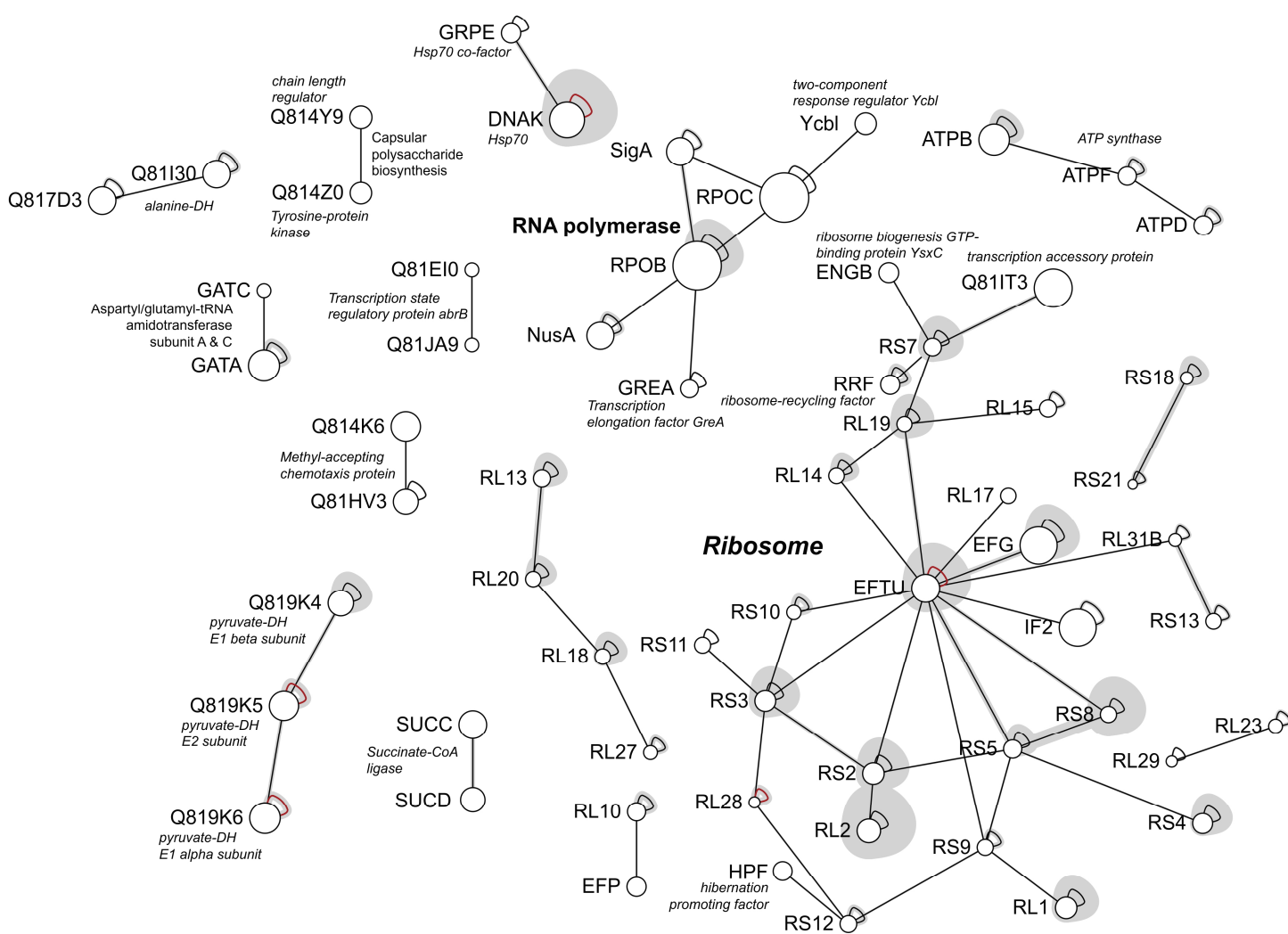

Supplemental figure 2: Cross-link interaction map of Bacillus subtilis (A) and cereus (B). Bacteria were grown in LB medium, harvested in exponential growth phase and cross-linked without prior cell disruption. Cross-links identified with the peptidefocused approach were manually checked, whether interactions are known, and were visualized with xiNET. 
5.1 Supplementary figures 

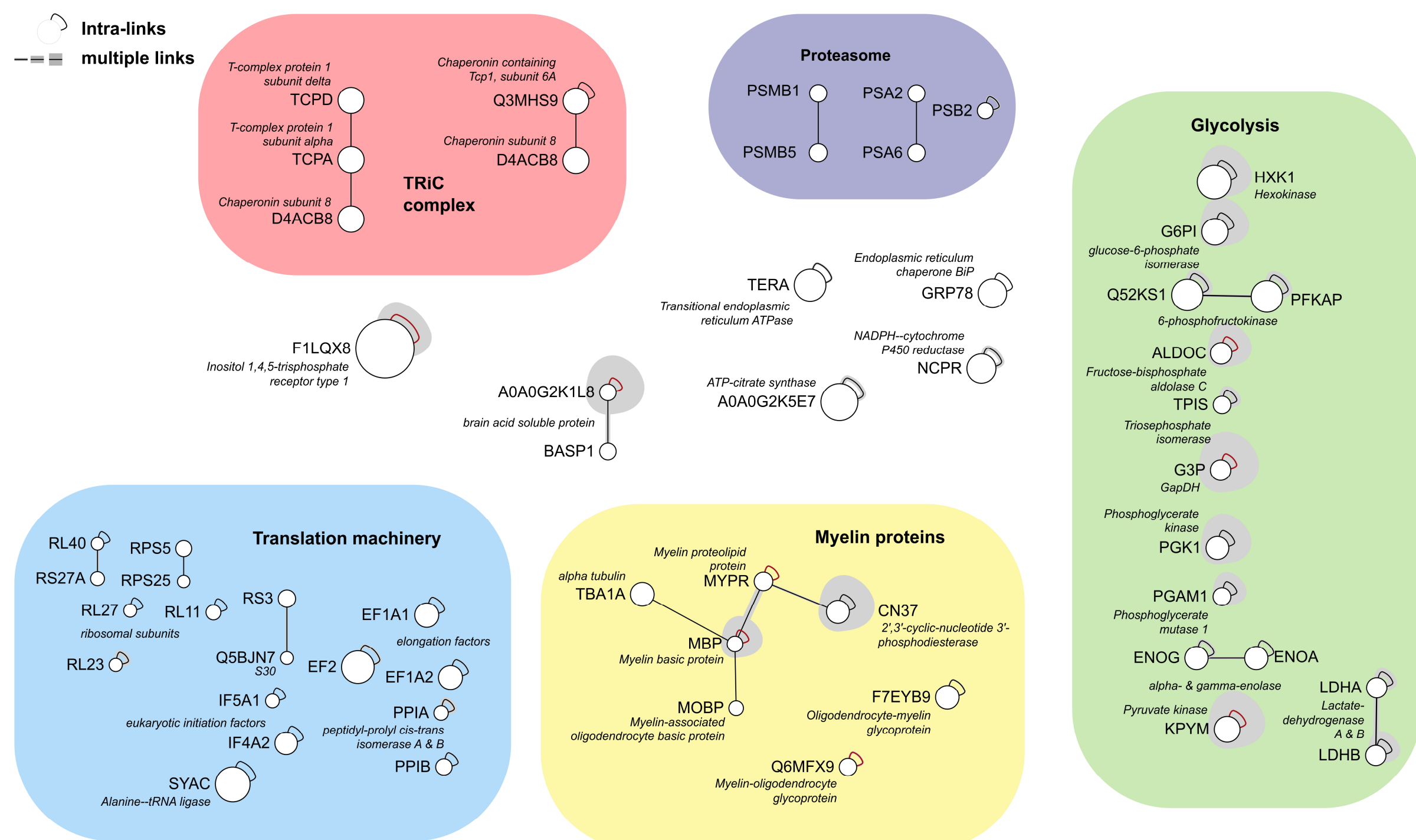

Supplemental figure 3: Cross-linking interaction map of identified unspecific proteins. Synaptosomes were prepared from rat brains, cross-linked and mitochondria were depleted after crosslinking. Samples were analyzed by LC-M S and cross-linked peptides were identified with plink 1 at $1 \%$ FDR with the peptide-focused approach. 


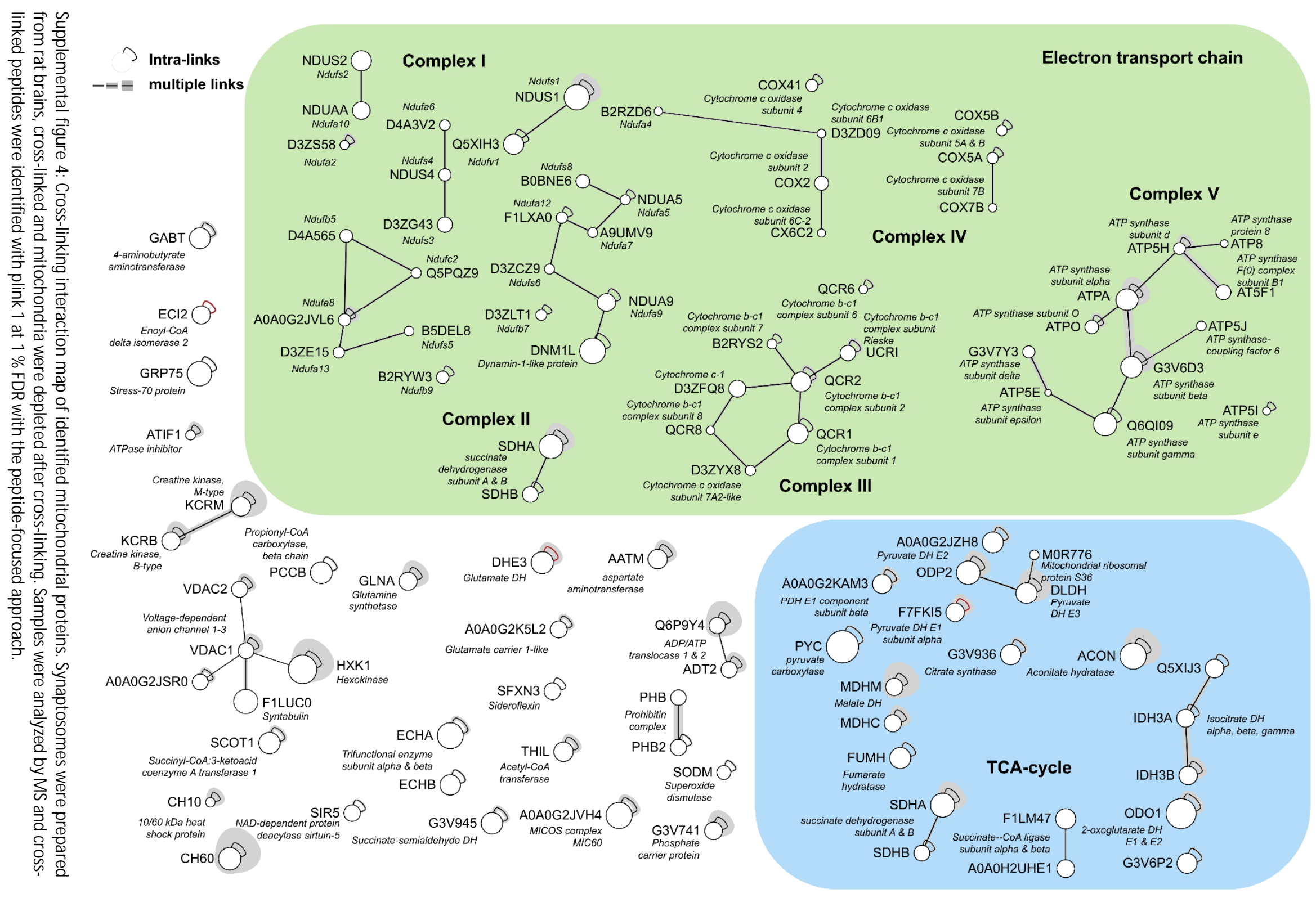




\subsection{Supplementary tables}

The CD-ROM enclosed to this work contains the following files:

\begin{tabular}{ll}
\hline File name & Description \\
\hline Supplementary table 1 & Identified cross-linked residues of in vivo cross-linked \\
& Bacillus subtilis and cereus \\
Supplementary table 2 & Label-free quantification analysis of mitochondria \\
& depleted synaptosome samples \\
Supplementary table 3 & Identified cross-linked residues of myelin- and \\
& mitochondria-depleted synaptosomes \\
Supplementary table 4 & Quantitative analysis of cross-linked residues of \\
& myelin- and mitochondria-depleted excited and \\
& resting synaptosomes \\
\hline
\end{tabular}




\section{References}

1. Fischer, L., Chen, Z.A. \& Rappsilber, J. Quantitative cross-linking/mass spectrometry using isotope-labelled cross-linkers. J Proteomics 88, 120-128 (2013).

2. Aebersold, R. \& Mann, M. M ass spectrometry-based proteomics. Nature 422, 198-207 (2003).

3. Aebersold, R. \& Mann, M. Mass-spectrometric exploration of proteome structure and function. Nature 537, 347-355 (2016).

4. Fenn, J.B., M ann, M., M eng, C.K., Wong, S.F. \& Whitehouse, C.M. Electrospray ionization for mass spectrometry of large biomolecules. Science 246, 64-71 (1989).

5. Hu, Q. et al. The Orbitrap: a new mass spectrometer. J M ass Spectrom 40, 430-443 (2005).

6. Michalski, A. et al. M ass spectrometry-based proteomics using $Q$ Exactive, a high-performance benchtop quadrupole Orbitrap mass spectrometer. Mol Cell Proteomics 10, M 111011015 (2011).

7. Kelstrup, C.D. et al. Performance Evaluation of the Q Exactive HF-X for Shotgun Proteomics. J Proteome Res 17, 727-738 (2018).

8. Eng, J.K., M cCormack, A.L. \& Yates, J.R. An approach to correlate tandem mass spectral data of peptides with amino acid sequences in a protein database. J Am Soc Mass Spectrom 5, 976989 (1994).

9. Cox, J. \& Mann, M. M axQuant enables high peptide identification rates, individualized p.p.b.range mass accuracies and proteome-wide protein quantification. Nat Biotechnol 26, 13671372 (2008).

10. Cox, J. et al. Andromeda: a peptide search engine integrated into the M axQuant environment. J Proteome Res 10, 1794-1805 (2011).

11. Elias, J.E., Haas, W., Faherty, B.K. \& Gygi, S.P. Comparative evaluation of mass spectrometry platforms used in large-scale proteomics investigations. Nat M ethods 2, 667-675 (2005).

12. Liu, T. et al. High dynamic range characterization of the trauma patient plasma proteome. Mol Cell Proteomics 5, 1899-1913 (2006).

13. Righetti, P.G., Castagna, A., Herbert, B., Reymond, F. \& Rossier, J.S. Prefractionation techniques in proteome analysis. Proteomics 3, 1397-1407 (2003).

14. Shevchenko, A., Tomas, H., Havlis, J., Olsen, J.V. \& Mann, M. In-gel digestion for mass spectrometric characterization of proteins and proteomes. Nat Protoc 1, 2856-2860 (2006).

15. Wang, Y. et al. Reversed-phase chromatography with multiple fraction concatenation strategy for proteome profiling of human M CF10A cells. Proteomics 11, 2019-2026 (2011).

16. Wang, H. \& Hanash, S. Multi-dimensional liquid phase based separations in proteomics. J Chromatogr B Analyt Technol Biomed Life Sci 787, 11-18 (2003).

17. Cox, J. et al. Accurate proteome-wide label-free quantification by delayed normalization and maximal peptide ratio extraction, termed MaxLFQ. M ol Cell Proteomics 13, 2513-2526 (2014).

18. Ong, S.E. \& Mann, M. M ass spectrometry-based proteomics turns quantitative. Nat Chem Biol 1, 252-262 (2005).

19. Ong, S.E. et al. Stable isotope labeling by amino acids in cell culture, SILAC, as a simple and accurate approach to expression proteomics. Mol Cell Proteomics 1, 376-386 (2002).

20. Bantscheff, M., Schirle, M., Sweetman, G., Rick, J. \& Kuster, B. Quantitative mass spectrometry in proteomics: a critical review. Anal Bioanal Chem 389, 1017-1031 (2007).

21. Thompson, A. et al. Tandem mass tags: a novel quantification strategy for comparative analysis of complex protein mixtures by M S/MS. Anal Chem 75, 1895-1904 (2003).

22. Gillet, L.C. et al. Targeted data extraction of the MS/MS spectra generated by dataindependent acquisition: a new concept for consistent and accurate proteome analysis. Mol Cell Proteomics 11, 0111016717 (2012).

23. Rappsilber, J. The beginning of a beautiful friendship: cross-linking/mass spectrometry and modelling of proteins and multi-protein complexes. J Struct Biol 173, 530-540 (2011). 
24. Peters, K. \& Richards, F.M. Chemical cross-linking: reagents and problems in studies of membrane structure. Annu Rev Biochem 46, 523-551 (1977).

25. Girshovich, A.S., Bochkareva, E.S. \& Vasiliev, V.D. Localization of elongation factor Tu on the ribosome. FEBS Lett 197, 192-198 (1986).

26. Sinz, A. Chemical cross-linking and mass spectrometry to map three-dimensional protein structures and protein-protein interactions. Mass Spectrom Rev 25, 663-682 (2006).

27. Leitner, A. et al. Chemical cross-linking/mass spectrometry targeting acidic residues in proteins and protein complexes. Proc Natl Acad Sci U S A 111, 9455-9460 (2014).

28. Singh, P., Panchaud, A. \& Goodlett, D.R. Chemical cross-linking and mass spectrometry as a low-resolution protein structure determination technique. Anal Chem 82, 2636-2642 (2010).

29. Rozbesky, D. et al. Impact of Chemical Cross-Linking on Protein Structure and Function. Anal Chem 90, 1104-1113 (2018).

30. Leitner, A., Walzthoeni, T. \& Aebersold, R. Lysine-specific chemical cross-linking of protein complexes and identification of cross-linking sites using LC-MS/MS and the XQuest/xProphet software pipeline. Nat Protoc 9, 120-137 (2014).

31. Rappsilber, J., Siniossoglou, S., Hurt, E.C. \& Mann, M. A generic strategy to analyze the spatial organization of multi-protein complexes by cross-linking and mass spectrometry. Anal Chem 72, 267-275 (2000).

32. Schmidt, C. \& Urlaub, H. Combining cryo-electron microscopy (cryo-EM) and cross-linking mass spectrometry (CX-MS) for structural elucidation of large protein assemblies. Curr Opin Struct Biol 46, 157-168 (2017).

33. Yan, C. et al. Structure of a yeast spliceosome at 3.6-angstrom resolution. Science 349, 11821191 (2015).

34. Chen, ZA. et al. Architecture of the RNA polymerase II-TFIIF complex revealed by cross-linking and mass spectrometry. EM BO J 29, 717-726 (2010).

35. Iacobucci, C. et al. First Community-Wide, Comparative Cross-Linking Mass Spectrometry Study. Anal Chem 91, 6953-6961 (2019).

36. Yilmaz, S. et al. Cross-linked peptide identification: A computational forest of algorithms. M ass Spectrom Rev 37, 738-749 (2018).

37. Tran, B.Q., Goodlett, D.R. \& Goo, Y.A. Advances in protein complex analysis by chemical crosslinking coupled with mass spectrometry (CXMS) and bioinformatics. Biochim Biophys Acta 1864, 123-129 (2016).

38. Maiolica, A. et al. Structural analysis of multiprotein complexes by cross-linking, mass spectrometry, and database searching. Mol Cell Proteomics 6, 2200-2211 (2007).

39. Panchaud, A., Singh, P., Shaffer, S.A. \& Goodlett, D.R. xComb: a cross-linked peptide database approach to protein-protein interaction analysis. J Proteome Res 9, 2508-2515 (2010).

40. Rinner, 0 . et al. Identification of cross-linked peptides from large sequence databases. Nat M ethods 5, 315-318 (2008).

41. Yang, B. et al. Identification of cross-linked peptides from complex samples. Nature methods 9, 904 (2012).

42. Hoopmann, M.R. et al. Kojak: efficient analysis of chemically cross-linked protein complexes. Journal of proteome research 14, 2190-2198 (2015).

43. Walzthoeni, T. et al. False discovery rate estimation for cross-linked peptides identified by mass spectrometry. Nat M ethods 9, 901-903 (2012).

44. lacobucci, C. \& Sinz, A. To Be or Not to Be? Five Guidelines to Avoid Misassignments in CrossLinking/M ass Spectrometry. Anal Chem 89, 7832-7835 (2017).

45. Merkley, E.D. et al. Distance restraints from crosslinking mass spectrometry: mining a molecular dynamics simulation database to evaluate lysine-lysine distances. Protein Sci 23, 747-759 (2014).

46. Kosinski, J. et al. Xlink Analyzer: software for analysis and visualization of cross-linking data in the context of three-dimensional structures. Journal of structural biology 189, 177-183 (2015).

47. Yilmaz, S. et al. Xilmass: A New Approach toward the Identification of Cross-Linked Peptides. Anal Chem 88, 9949-9957 (2016). 
48. Kao, A. et al. Development of a novel cross-linking strategy for fast and accurate identification of cross-linked peptides of protein complexes. M ol Cell Proteomics 10, M 110002212 (2011).

49. Liu, F., Rijkers, D.T., Post, H. \& Heck, A.J. Proteome-wide profiling of protein assemblies by cross-linking mass spectrometry. Nat M ethods 12, 1179-1184 (2015).

50. Syka, J.E., Coon, J.J., Schroeder, M.J., Shabanowitz, J. \& Hunt, D.F. Peptide and protein sequence analysis by electron transfer dissociation mass spectrometry. Proc Natl Acad Sci U S A 101, 9528-9533 (2004).

51. Liu, F., Lossl, P., Scheltema, R., Viner, R. \& Heck, A.J.R. Optimized fragmentation schemes and data analysis strategies for proteome-wide cross-link identification. Nat Commun 8, 15473 (2017).

52. Liu, F., Lossl, P., Rabbitts, B.M., Balaban, R.S. \& Heck, A.J.R. The interactome of intact mitochondria by cross-linking mass spectrometry provides evidence for coexisting respiratory supercomplexes. Mol Cell Proteomics 17, 216-232 (2018).

53. Fasci, D., van Ingen, H., Scheltema, R.A. \& Heck, A.J.R. Histone Interaction Landscapes Visualized by Crosslinking Mass Spectrometry in Intact Cell Nuclei. Mol Cell Proteomics 17, 2018-2033 (2018).

54. Chen, Z.A. \& Rappsilber, J. Protein Dynamics in Solution by Quantitative Crosslinking/M ass Spectrometry. Trends Biochem Sci 43, 908-920 (2018).

55. Schmidt, $C$. et al. Comparative cross-linking and mass spectrometry of an intact F-type ATPase suggest a role for phosphorylation. Nat Commun 4, 1985 (2013).

56. Chen, Z.A., Fischer, L., Cox, J. \& Rappsilber, J. Quantitative Cross-linking/Mass Spectrometry Using Isotope-labeled Cross-linkers and M axQuant. M ol Cell Proteomics 15, 2769-2778 (2016).

57. Muller, F., Fischer, L., Chen, Z.A., Auchynnikava, T. \& Rappsilber, J. On the Reproducibility of Label-Free Quantitative Cross-Linking/M ass Spectrometry. J Am Soc M ass Spectrom 29, 405412 (2018).

58. Muller, F., Kolbowski, L., Bernhardt, O.M., Reiter, L. \& Rappsilber, J. Data-independent Acquisition Improves Quantitative Cross-linking Mass Spectrometry. Molecular \& Cellular Proteomics 18, 786-795 (2019).

59. Yu, C. et al. Developing a Multiplexed Quantitative Cross-Linking M ass Spectrometry Platform for Comparative Structural Analysis of Protein Complexes. Anal Chem 88, 10301-10308 (2016).

60. Chavez, J.D. et al. Quantitative interactome analysis reveals a chemoresistant edgotype. Nat Commun 6, 7928 (2015).

61. Chavez, J.D., Schweppe, D.K., Eng, J.K. \& Bruce, J.E. In Vivo Conformational Dynamics of Hsp90 and Its Interactors. Cell Chem Biol 23, 716-726 (2016).

62. Rutecki, P.A. Neuronal excitability: voltage-dependent currents and synaptic transmission. J Clin Neurophysiol 9, 195-211 (1992).

63. Chua, J.J., Kindler, S., Boyken, J. \& Jahn, R. The architecture of an excitatory synapse. J Cell Sci 123, 819-823 (2010).

64. Schoch, S. \& Gundelfinger, E.D. Molecular organization of the presynaptic active zone. Cell Tissue Res 326, 379-391 (2006).

65. Owens, D.F. \& Kriegstein, A.R. Is there more to GABA than synaptic inhibition? Nat Rev Neurosci 3, 715-727 (2002).

66. Landis, D.M., Hall, A.K., Weinstein, L.A. \& Reese, T.S. The organization of cytoplasm at the presynaptic active zone of a central nervous system synapse. Neuron 1, $201-209$ (1988).

67. Jahne, S., Rizzoli, S.O. \& Helm, M.S. The structure and function of presynaptic endosomes. Exp Cell Res 335, 172-179 (2015).

68. Ly, C.V. \& Verstreken, P. M itochondria at the synapse. Neuroscientist 12, 291-299 (2006).

69. $W u, Y$. et al. Contacts between the endoplasmic reticulum and other membranes in neurons. Proc Natl Acad Sci U S A 114, E4859-E4867 (2017).

70. Bouchard, R., Pattarini, R. \& Geiger, J.D. Presence and functional significance of presynaptic ryanodine receptors. Prog Neurobiol 69, 391-418 (2003). 
71. Terasaki, M., Slater, N.T., Fein, A., Schmidek, A. \& Reese, T.S. Continuous network of endoplasmic reticulum in cerebellar Purkinje neurons. Proc Natl Acad Sci U S A 91, 7510-7514 (1994).

72. Sudhof, T.C. The synaptic vesicle cycle. Annu Rev Neurosci 27, 509-547 (2004).

73. Rizzoli, S.O. \& Betz, W.J. Synaptic vesicle pools. Nat Rev Neurosci 6, 57-69 (2005).

74. Rizzoli, S.O. \& Jahn, R. Kiss-and-run, collapse and 'readily retrievable' vesicles. Traffic 8, 11371144 (2007).

75. Takamori, S. et al. M olecular anatomy of a trafficking organelle. Cell 127, 831-846 (2006).

76. Inoue, T. et al. Structure and regulation of the V-ATPases. J Bioenerg Biomembr 37, 393-398 (2005).

77. Hilfiker, S. et al. Synapsins as regulators of neurotransmitter release. Philos Trans R Soc Lond B Biol Sci 354, 269-279 (1999).

78. Wiedenmann, B. \& Franke, W.W. Identification and localization of synaptophysin, an integral membrane glycoprotein of $\mathrm{M} \mathrm{r}$ 38,000 characteristic of presynaptic vesicles. Cell 41, 1017-1028 (1985).

79. Zhang, X., Rizo, J. \& Sudhof, T.C. M echanism of phospholipid binding by the C2A-domain of synaptotagmin I. Biochemistry 37, 12395-12403 (1998).

80. Sudhof, T.C. The presynaptic active zone. Neuron 75, 11-25 (2012).

81. Mukherjee, $\mathrm{K}$. et al. Piccolo and bassoon maintain synaptic vesicle clustering without directly participating in vesicle exocytosis. Proc Natl Acad Sci U S A 107, 6504-6509 (2010).

82. Hallermann, S. et al. Bassoon speeds vesicle reloading at a central excitatory synapse. Neuron 68, 710-723 (2010).

83. Dulubova, I. et al. A M unc13/RIM/Rab3 tripartite complex: from priming to plasticity? EM BO J 24, 2839-2850 (2005).

84. Kaeser, P.S. et al. RIM proteins tether Ca2+channels to presynaptic active zones via a direct PDZ-domain interaction. Cell 144, 282-295 (2011).

85. Butz, S., Okamoto, M. \& Sudhof, T.C. A tripartite protein complex with the potential to couple synaptic vesicle exocytosis to cell adhesion in brain. Cell 94, 773-782 (1998).

86. Sutton, R.B., Fasshauer, D., Jahn, R. \& Brunger, A.T. Crystal structure of a SNARE complex involved in synaptic exocytosis at 2.4 A resolution. Nature 395, 347-353 (1998).

87. Sollner, T., Bennett, M.K., Whiteheart, S.W., Scheller, R.H. \& Rothman, J.E. A protein assemblydisassembly pathway in vitro that may correspond to sequential steps of synaptic vesicle docking, activation, and fusion. Cell 75, 409-418 (1993).

88. Burkhardt, P., Hattendorf, D.A., Weis, W.I. \& Fasshauer, D. M unc18a controls SNARE assembly through its interaction with the syntaxin N-peptide. EM BO J 27, 923-933 (2008).

89. Ma, C., Li, W., Xu, Y. \& Rizo, J. Munc13 mediates the transition from the closed syntaxinM unc18 complex to the SNARE complex. Nat Struct M ol Biol 18, 542-549 (2011).

90. Zhou, Q. et al. The primed SNARE-complexin-synaptotagmin complex for neuronal exocytosis. Nature 548, 420-425 (2017).

91. Sudhof, T.C. Neurotransmitter release: the last millisecond in the life of a synaptic vesicle. Neuron 80, 675-690 (2013).

92. Rajappa, R., Gauthier-Kemper, A., Boning, D., Huve, J. \& Klingauf, J. Synaptophysin 1 Clears Synaptobrevin 2 from the Presynaptic Active Zone to Prevent Short-Term Depression. Cell Rep 14, 1369-1381 (2016).

93. Soykan, T., M aritzen, T. \& Haucke, V. M odes and mechanisms of synaptic vesicle recycling. Curr Opin Neurobiol 39, 17-23 (2016).

94. Ungewickell, E.J. \& Hinrichsen, L. Endocytosis: clathrin-mediated membrane budding. Curr Opin Cell Biol 19, 417-425 (2007).

95. Verstreken, P. et al. Synaptojanin is recruited by endophilin to promote synaptic vesicle uncoating. Neuron 40, 733-748 (2003).

96. Bocking, T., Aguet, F., Harrison, S.C. \& Kirchhausen, T. Single-molecule analysis of a molecular disassemblase reveals the mechanism of Hsc70-driven clathrin uncoating. Nat Struct M ol Biol 18, 295-301 (2011). 
97. Hoopmann, P. et al. Endosomal sorting of readily releasable synaptic vesicles. Proc Natl Acad Sci U SA 107, 19055-19060 (2010).

98. Zhu, S. \& Gouaux, E. Structure and symmetry inform gating principles of ionotropic glutamate receptors. Neuropharmacology 112, 11-15 (2017).

99. Chin, D. \& M eans, A.R. Calmodulin: a prototypical calcium sensor. Trends Cell Biol 10, 322-328 (2000).

100. Liu, Y.C. \& Storm, D.R. Regulation of free calmodulin levels by neuromodulin: neuron growth and regeneration. Trends Pharmacol Sci 11, 107-111 (1990).

101. Kennedy, M.B., Beale, H.C., Carlisle, H.J. \& Washburn, L.R. Integration of biochemical signalling in spines. Nat Rev Neurosci 6, 423-434 (2005).

102. Myers, J.B. et al. The CaM KII holoenzyme structure in activation-competent conformations. Nat Commun 8, 15742 (2017).

103. Dillon, C. \& Goda, Y. The actin cytoskeleton: integrating form and function at the synapse. Annu Rev Neurosci 28, 25-55 (2005).

104. Xu, K., Zhong, G. \& Zhuang, X. Actin, spectrin, and associated proteins form a periodic cytoskeletal structure in axons. Science 339, 452-456 (2013).

105. Goldstein, L.S. \& Yang, Z. M icrotubule-based transport systems in neurons: the roles of kinesins and dyneins. Annu Rev Neurosci 23, 39-71 (2000).

106. Murakoshi, H., Wang, H. \& Yasuda, R. Local, persistent activation of Rho GTPases during plasticity of single dendritic spines. Nature 472, 100-104 (2011).

107. Merriam, E.B. et al. Synaptic regulation of microtubule dynamics in dendritic spines by calcium, F-actin, and drebrin. J Neurosci 33, 16471-16482 (2013).

108. Simonovic, M., Zhang, Z., Cianci, C.D., Steitz, T.A. \& M orrow, J.S. Structure of the calmodulin alphall-spectrin complex provides insight into the regulation of cell plasticity. J Biol Chem 281, 34333-34340 (2006).

109. Periasamy, M \& \& Kalyanasundaram, A. SERCA pump isoforms: their role in calcium transport and disease. Muscle Nerve 35, 430-442 (2007).

110. Toyoshima, C., Nakasako, M., Nomura, H. \& Ogawa, H. Crystal structure of the calcium pump of sarcoplasmic reticulum at 2.6 A resolution. Nature $405,647-655$ (2000).

111. Olesen, $C$. et al. The structural basis of calcium transport by the calcium pump. Nature $\mathbf{4 5 0}$, 1036-1042 (2007).

112. Toyoshima, C. et al. Crystal structures of the calcium pump and sarcolipin in the $\mathrm{Mg} 2+$-bound E1 state. Nature 495, 260-264 (2013).

113. Kwon, S.K. et al. LKB1 Regulates M itochondria-Dependent Presynaptic Calcium Clearance and Neurotransmitter Release Properties at Excitatory Synapses along Cortical Axons. PLoS Biol 14, e1002516 (2016).

114. Fan, G. et al. Cryo-EM reveals ligand induced allostery underlying InsP3R channel gating. Cell Res 28, 1158-1170 (2018).

115. Kanai, R., Ogawa, H., Vilsen, B., Cornelius, F. \& Toyoshima, C. Crystal structure of a Na+bound $\mathrm{Nat}, \mathrm{K}+$ ATPase preceding the E1P state. Nature 502, 201 (2013).

116. Hebb, C.O.\& Whittaker, V.P. Intracellular distributions of acetylcholine and choline acetylase. J Physiol 142, 187-196 (1958).

117. Whittaker, V.P. Thirty years of synaptosome research. J Neurocytol 22, 735-742 (1993).

118. Gray, E.G. \& Whittaker, V.P. The isolation of nerve endings from brain: an electron-microscopic study of cell fragments derived by homogenization and centrifugation. J Anat 96, 79-88 (1962).

119. Fischer von Mollard, G., Sudhof, T.C. \& Jahn, R. A small GTP-binding protein dissociates from synaptic vesicles during exocytosis. Nature 349, 79-81 (1991).

120. Wilhelm, B.G. et al. Composition of isolated synaptic boutons reveals the amounts of vesicle trafficking proteins. Science 344, 1023-1028 (2014).

121. Barondes, S.H. Synaptic Macromolecules - Identification and Metabolism. Annual Review of Biochemistry 43, 147-168 (1974). 
122. Ahnert-Hilger, G., Bhakdi, S. \& Gratzl, M. M inimal requirements for exocytosis. A study using PC 12 cells permeabilized with staphylococcal alpha-toxin. J Biol Chem 260, 12730-12734 (1985).

123. Gurd, J.W., Jones, L.R., M ahler, H.R. \& M oore, W.J. Isolation and partial characterization of rat brain synaptic plasma membranes. J Neurochem 22, 281-290 (1974).

124. Sydow, J.F. et al. Structural basis of transcription: mismatch-specific fidelity mechanisms and paused RNA polymerase II with frayed RNA. M ol Cell 34, 710-721 (2009).

125. Schilbach, S. et al. Structures of transcription pre-initiation complex with TFIIH and M ediator. Nature 551, 204-209 (2017).

126. Schrader, J. et al. The inhibition mechanism of human $20 \mathrm{~S}$ proteasomes enables nextgeneration inhibitor design. Science 353, 594-598 (2016).

127. Wessel, D. \& Flugge, U.I. A method for the quantitative recovery of protein in dilute solution in the presence of detergents and lipids. Anal Biochem 138, 141-143 (1984).

128. Milo, R. What is the total number of protein molecules per cell volume? A call to rethink some published values. Bioessays 35, 1050-1055 (2013).

129. M ilo, R., Jorgensen, P., M oran, U., Weber, G. \& Springer, M. BioNumbers--the database of key numbers in molecular and cell biology. Nucleic Acids Res 38, D750-753 (2010).

130. Boyken, J. et al. M olecular profiling of synaptic vesicle docking sites reveals novel proteins but few differences between glutamatergic and GABAergic synapses. Neuron 78, 285-297 (2013).

131. Carlin, R.K., Grab, D.J., Cohen, R.S. \& Siekevitz, P. Isolation and characterization of postsynaptic densities from various brain regions: enrichment of different types of postsynaptic densities. J Cell Biol 86, 831-845 (1980).

132. Schmidt, R. \& Sinz, A. Improved single-step enrichment methods of cross-linked products for protein structure analysis and protein interaction mapping. Anal Bioanal Chem 409, 2393-2400 (2017).

133. Leitner, A. et al. Expanding the chemical cross-linking toolbox by the use of multiple proteases and enrichment by size exclusion chromatography. Mol Cell Proteomics 11, M 111014126 (2012).

134. Rappsilber, J., Mann, M.\& Ishihama, Y. Protocol for micro-purification, enrichment, prefractionation and storage of peptides for proteomics using StageTips. Nat Protoc 2, 1896-1906 (2007).

135. Cox, J. \& Mann, M. M axQuant enables high peptide identification rates, individualized p.p.b.range mass accuracies and proteome-wide protein quantification. Nature Biotechnology 26, 1367 (2008).

136. MacCoss, M.J., Noble, W.S. \& Käll, L. Fast and accurate protein false discovery rates on largescale proteomics data sets with percolator 3.0. Journal of the American Society for Mass Spectrometry 27, 1719-1727 (2016).

137. Combe, C.W., Fischer, L. \& Rappsilber, J. xiNET: cross-link network maps with residue resolution. M olecular \& Cellular Proteomics, mcp. 0114. 042259 (2015).

138. Banerjee, S. et al. 2.3 A resolution cryo-EM structure of human p97 and mechanism of allosteric inhibition. Science 351, 871-875 (2016).

139. Zhang, W., M arzilli, L.A., Rouse, J.C. \& Czupryn, M.J. Complete disulfide bond assignment of a recombinant immunoglobulin G4 monoclonal antibody. Anal Biochem 311, 1-9 (2002).

140. Trnka, M.J., Baker, P.R., Robinson, P.J., Burlingame, A.L. \& Chalkley, R.J. M atching cross-linked peptide spectra: only as good as the worse identification. Mol Cell Proteomics 13, 420-434 (2014).

141. de Jong, L. et al. In-Culture Cross-Linking of Bacterial Cells Reveals Large-Scale Dynamic Protein-Protein Interactions at the Peptide Level. J Proteome Res 16, 2457-2471 (2017).

142. Tao, H., Bausch, C., Richmond, C., Blattner, F.R. \& Conway, T. Functional genomics: expression analysis of Escherichia coli growing on minimal and rich media. J Bacteriol 181, 6425-6440 (1999).

143. Loveland, A.B., Demo, G., Grigorieff, N. \& Korostelev, A.A. Ensemble cryo-EM elucidates the mechanism of translation fidelity. Nature 546, 113-117 (2017). 
144. De Pinto, V., M essina, A., Lane, D.J. \& Lawen, A. Voltage-dependent anion-selective channel (VDAC) in the plasma membrane. FEBS Lett 584, 1793-1799 (2010).

145. Jackson, L.P. et al. A large-scale conformational change couples membrane recruitment to cargo binding in the AP2 clathrin adaptor complex. Cell 141, 1220-1229 (2010).

146. Gong, D. et al. Structure of the human plasma membrane $\mathrm{Ca}(2+)-A T P a s e ~ 1$ in complex with its obligatory subunit neuroplastin. Nat Commun 9, 3623 (2018).

147. Brodin, P., Falchetto, R., Vorherr, T. \& Carafoli, E. Identification of two domains which mediate the binding of activating phospholipids to the plasma-membrane Ca2+pump. Eur J Biochem 204, 939-946 (1992).

148. Shinoda, T., Ogawa, H., Cornelius, F. \& Toyoshima, C. Crystal structure of the sodiumpotassium pump at $2.4 \AA$ resolution. Nature 459,446 (2009).

149. Donnet, C., Arystarkhova, E. \& Sweadner, K.J. Thermal denaturation of the Na,K-ATPase provides evidence for alpha-alpha oligomeric interaction and gamma subunit association with the C-terminal domain. J Biol Chem 276, 7357-7365 (2001).

150. Fan, G. et al. Gating machinery of InsP3R channels revealed by electron cryomicroscopy. Nature 527, 336-341 (2015).

151. Chao, L.H. et al. A mechanism for tunable autoinhibition in the structure of a human Ca2+/calmodulin- dependent kinase II holoenzyme. Cell 146, 732-745 (2011).

152. Yamagata, Y. et al. Kinase-dead knock-in mouse reveals an essential role of kinase activity of $\mathrm{Ca} 2+/$ calmodulin-dependent protein kinase llalpha in dendritic spine enlargement, long-term potentiation, and learning. J Neurosci 29, 7607-7618 (2009).

153. Godbout, M. et al. 1G5: a calmodulin-binding, vesicle-associated, protein kinase-like protein enriched in forebrain neurites. J Neurosci 14, 1-13 (1994).

154. Liang, Z. et al. The pseudokinase CaM Kv is required for the activity-dependent maintenance of dendritic spines. Nat Commun 7, 13282 (2016).

155. Leto, T.L., Pleasic, S., Forget, B.G., Benz, E.J., Jr. \& Marchesi, V.T. Characterization of the calmodulin-binding site of nonerythroid alpha-spectrin. Recombinant protein and model peptide studies. J Biol Chem 264, 5826-5830 (1989).

156. Dubreuil, R.R., Brandin, E., Reisberg, J.H., Goldstein, L.S. \& Branton, D. Structure, calmodulinbinding, and calcium-binding properties of recombinant alpha spectrin polypeptides. J Biol Chem 266, 7189-7193 (1991).

157. Ursitti, J.A., Kotula, L., DeSilva, T.M., Curtis, P.J. \& Speicher, D.W. Mapping the human erythrocyte beta-spectrin dimer initiation site using recombinant peptides and correlation of its phasing with the alpha-actinin dimer site. J Biol Chem 271, 6636-6644 (1996).

158. Zhou, Q. et al. Architecture of the synaptotagmin-SNARE machinery for neuronal exocytosis. Nature 525, 62-67 (2015).

159. Avila-Sakar, A.J., Creutz, C.E. \& Kretsinger, R.H. Crystal structure of bovine annexin VI in a calcium-bound state. Biochim Biophys Acta 1387, 103-116 (1998).

160. Benz, J. et al. The structure of recombinant human annexin VI in crystals and membranebound. J M ol Biol 260, 638-643 (1996).

161. Huber, R., Schneider, M., Mayr, I., Romisch, J. \& Paques, E.P. The calcium binding sites in human annexin $\mathrm{V}$ by crystal structure analysis at $2.0 \mathrm{~A}$ resolution. Implications for membrane binding and calcium channel activity. FEBS Lett 275, 15-21 (1990).

162. Nakamura, K. et al. Functional specialization of calreticulin domains. J Cell Biol 154, 961-972 (2001).

163. Hubbard, M.J., M angum, J.E. \& M CHugh, N.J. Purification and biochemical characterization of native ERp29 from rat liver. Biochem J 383, 589-597 (2004).

164. Georgopoulou, N. et al. BM 88 is a dual function molecule inducing cell cycle exit and neuronal differentiation of neuroblastoma cells via cyclin D1 down-regulation and retinoblastoma protein hypophosphorylation. J Biol Chem 281, 33606-33620 (2006).

165. Masgrau, R. et al. BM 88/Cend1 regulates stimuli-induced intracellular calcium mobilization. Neuropharmacology 56, 598-609 (2009). 
166. Patsavoudi, E. et al. Characterization and localization of the BM 88 antigen in the developing and adult rat brain. J Neurosci Res 40, 506-518 (1995).

167. Verbisck, N.V. et al. ADAM 23 negatively modulates alpha(v)beta(3) integrin activation during metastasis. Cancer Res 69, 5546-5552 (2009).

168. Bayrhuber, M. et al. Structure of the human voltage-dependent anion channel. Proc Natl Acad Sci U SA 105, 15370-15375 (2008).

169. Ujwal, R. et al. The crystal structure of mouse VDAC1 at 2.3 A resolution reveals mechanistic insights into metabolite gating. Proc Natl Acad Sci U SA 105, 17742-17747 (2008).

170. Hiller, S. et al. Solution structure of the integral human membrane protein VDAC-1 in detergent micelles. Science 321, 1206-1210 (2008).

171. Choudhary, O.P. et al. Structure-guided simulations illuminate the mechanism of ATP transport through VDAC1. Nat Struct M ol Biol 21, 626-632 (2014).

172. O'Gorman, E. et al. The role of creatine kinase in inhibition of mitochondrial permeability transition. FEBS Lett 414, 253-257 (1997).

173. Schlattner, U. et al. C-terminal lysines determine phospholipid interaction of sarcomeric mitochondrial creatine kinase. J Biol Chem 279, 24334-24342 (2004).

174. Gotze, M. et al. StavroX--a software for analyzing crosslinked products in protein interaction studies. J Am Soc Mass Spectrom 23, 76-87 (2012).

175. Cagney, G., Amiri, S., Premawaradena, T., Lindo, M. \& Emili, A. In silico proteome analysis to facilitate proteomics experiments using mass spectrometry. Proteome Sci 1, 5 (2003).

176. Buncherd, $\mathrm{H}$. et al. Isolation of cross-linked peptides by diagonal strong cation exchange chromatography for protein complex topology studies by peptide fragment fingerprinting from large sequence databases. J Chromatogr A 1348, 34-46 (2014).

177. Lu, S. et al. Mapping native disulfide bonds at a proteome scale. Nat M ethods $\mathbf{1 2}$, 329-331 (2015).

178. Diaz, R., Mayorga, L.S., Weidman, P.J., Rothman, J.E. \& Stahl, P.D. Vesicle fusion following receptor-mediated endocytosis requires a protein active in Golgi transport. Nature 339, 398400 (1989).

179. Otto, H., Hanson, P.I. \& Jahn, R. Assembly and disassembly of a ternary complex of synaptobrevin, syntaxin, and SNAP-25 in the membrane of synaptic vesicles. Proc Natl Acad Sci U S A 94, 6197-6201 (1997).

180. Ding, Y.H. et al. Increasing the Depth of Mass-Spectrometry-Based Structural Analysis of Protein Complexes through the Use of Multiple Cross-Linkers. Anal Chem 88, 4461-4469 (2016).

181. Lu, L. et al. Identification of MS-Cleavable and Noncleavable Chemically Cross-Linked Peptides with M etaM orpheus. J Proteome Res 17, 2370-2376 (2018).

182. Senko, M.W. et al. Novel parallelized quadrupole/linear ion trap/Orbitrap tribrid mass spectrometer improving proteome coverage and peptide identification rates. Anal Chem $\mathbf{8 5}$, 11710-11714 (2013).

183. Yu, C., Kandur, W., Kao, A., Rychnovsky, S. \& Huang, L. Developing new isotope-coded mass spectrometry-cleavable cross-linkers for elucidating protein structures. Anal Chem $\mathbf{8 6}, \mathbf{2 0 9 9 -}$ 2106 (2014).

184. Lima, D.B. et al. SIM-XL: A powerful and user-friendly tool for peptide cross-linking analysis. J Proteomics 129, 51-55 (2015).

185. Langer, J.A. \& Lake, J.A. Elongation factor Tu localized on the exterior surface of the small ribosomal subunit. J Mol Biol 187, 617-621 (1986).

186. Perkins, G.A. et al. The micro-architecture of mitochondria at active zones: electron tomography reveals novel anchoring scaffolds and cristae structured for high-rate metabolism. J Neurosci 30, 1015-1026 (2010).

187. Srinivasan, Y., Elmer, L., Davis, J., Bennett, V. \& Angelides, K. Ankyrin and spectrin associate with voltage-dependent sodium channels in brain. Nature 333, 177-180 (1988).

188. Nelson, W.J. \& Veshnock, P.J. Ankyrin binding to (Na++K+)ATPase and implications for the organization of membrane domains in polarized cells. Nature 328, 533-536 (1987). 
189. Cantiello, H.F. Actin filaments stimulate the $\mathrm{Na(+)-K(+)-ATPase.} \mathrm{Am} \mathrm{J} \mathrm{Physiol} \mathrm{269,} \mathrm{F637-643}$ (1995).

190. Dulubova, I. et al. Munc18-1 binds directly to the neuronal SNARE complex. Proc Natl Acad Sci U SA 104, 2697-2702 (2007).

191. Chen, X. et al. Three-dimensional structure of the complexin/SNARE complex. Neuron 33, 397409 (2002).

192. Fenster, S.D. et al. Piccolo, a presynaptic zinc finger protein structurally related to bassoon. Neuron 25, 203-214 (2000).

193. Limbach, C. et al. M olecular in situ topology of Aczonin/Piccolo and associated proteins at the mammalian neurotransmitter release site. Proc Natl Acad Sci U SA 108, E392-401 (2011).

194. Leonoudakis, D., Conti, L.R., Radeke, C.M., M cGuire, L.M \& \& Vandenberg, C.A. A multiprotein trafficking complex composed of SAP97, CASK, Veli, and Mint1 is associated with inward rectifier Kir2 potassium channels. J Biol Chem 279, 19051-19063 (2004).

195. Olsen, O. et al. Neurotransmitter release regulated by a MALS-liprin-alpha presynaptic complex. J Cell Biol 170, 1127-1134 (2005).

196. Olsen, O., Moore, K.A., Nicoll, R.A. \& Bredt, D.S. Synaptic transmission regulated by a presynaptic M ALS/Liprin-alpha protein complex. Curr Opin Cell Biol 18, 223-227 (2006).

197. Cipriano, D.J. et al. Processive ATP-driven substrate disassembly by the N-ethylmaleimidesensitive factor (NSF) molecular machine. J Biol Chem 288, 23436-23445 (2013).

198. Schweppe, D.K. et al. M itochondrial protein interactome elucidated by chemical cross-linking mass spectrometry. Proc Natl Acad Sci U S A 114, 1732-1737 (2017).

199. Tang, X., M unske, G.R., Siems, W.F. \& Bruce, J.E. M ass spectrometry identifiable cross-linking strategy for studying protein-protein interactions. Anal Chem 77, 311-318 (2005).

200. Sepp, M. et al. Tight coupling of $\mathrm{Na}+\mathrm{K}+\mathrm{ATPase}$ with glycolysis demonstrated in permeabilized rat cardiomyocytes. PLoS One $\mathbf{9}$, e99413 (2014).

201. Ikemoto, A., Bole, D.G. \& Ueda, T. Glycolysis and glutamate accumulation into synaptic vesicles. Role of glyceraldehyde phosphate dehydrogenase and 3-phosphoglycerate kinase. J Biol Chem 278, 5929-5940 (2003).

202. Kinoshita, A., Noda, M . \& Kinoshita, M. Differential localization of septins in the mouse brain. J Comp Neurol 428, 223-239 (2000).

203. Xie, Y. et al. The GTP-binding protein Septin 7 is critical for dendrite branching and dendriticspine morphology. Curr Biol 17, 1746-1751 (2007).

204. Fernandez-M oya, S.M., Bauer, K.E. \& Kiebler, M.A. M eet the players: local translation at the synapse. Front M ol Neurosci 7, 84 (2014).

205. Jung, H., Gkogkas, C.G., Sonenberg, N. \& Holt, C.E. Remote control of gene function by local translation. Cell 157, 26-40 (2014).

206. Pohlmann, T., Baumann, S., Haag, C., Albrecht, M . \& Feldbrugge, M . A FYVE zinc finger domain protein specifically links mRNA transport to endosome trafficking. Elife 4 (2015).

207. Li, S. et al. Septin 7 is required for orderly meiosis in mouse oocytes. Cell Cycle 11, 3211-3218 (2012).

208. Fukata, Y. et al. Epilepsy-related ligand/receptor complex LGI1 and ADAM 22 regulate synaptic transmission. Science 313, 1792-1795 (2006).

209. Rios, J.C. et al. Contactin-associated protein (Caspr) and contactin form a complex that is targeted to the paranodal junctions during myelination. J Neurosci 20, 8354-8364 (2000).

210. Berglund, E.O. et al. Ataxia and abnormal cerebellar microorganization in mice with ablated contactin gene expression. Neuron 24, 739-750 (1999).

211. Deng, Y. et al. N-M yc downstream-regulated gene 2 (NDRG2) inhibits glioblastoma cell proliferation. Int J Cancer 106, 342-347 (2003).

212. Hwang, J. et al. Crystal structure of the human N-M yc downstream-regulated gene 2 protein provides insight into its role as a tumor suppressor. J Biol Chem 286, 12450-12460 (2011).

213. Kalaydjieva, L. et al. N-myc downstream-regulated gene 1 is mutated in hereditary motor and sensory neuropathy-Lom. Am J Hum Genet 67, 47-58 (2000). 
214. Takahashi, K., Yamada, M., Ohata, H., Honda, K. \& Yamada, M. Ndrg2 promotes neurite outgrowth of NGF-differentiated PC12 cells. Neurosci Lett 388, 157-162 (2005).

215. M hawech, P. 14-3-3 proteins--an update. Cell Res 15, 228-236 (2005).

216. Berg, D., Holzmann, C. \& Riess, 0. 14-3-3 proteins in the nervous system. Nat Rev Neurosci 4, 752-762 (2003).

217. Efendiev, R. et al. The 14-3-3 protein translates the NA+,K+ATPase \{alpha\}1-subunit phosphorylation signal into binding and activation of phosphoinositide 3-kinase during endocytosis. J Biol Chem 280, 16272-16277 (2005).

218. Lambright, D.G. et al. The 2.0 A crystal structure of a heterotrimeric G protein. Nature 379, 311-319 (1996).

219. Brown, A.M . \& Birnbaumer, L. Direct G protein gating of ion channels. Am J Physiol 254, H401410 (1988).

220. Heydorn, A. et al. A library of 7TM receptor C-terminal tails. Interactions with the proposed post-endocytic sorting proteins ERM -binding phosphoprotein 50 (EBP50), N-ethylmaleimidesensitive factor (NSF), sorting nexin 1 (SNX1), and G protein-coupled receptor-associated sorting protein (GASP). J Biol Chem 279, 54291-54303 (2004).

221. Krasnoperov, V.G. et al. alpha-Latrotoxin stimulates exocytosis by the interaction with a neuronal G-protein-coupled receptor. Neuron 18, 925-937 (1997).

222. Jarvis, S.E., M agga, J.M., Beedle, A.M ., Braun, J.E. \& Zamponi, G.W. G protein modulation of Ntype calcium channels is facilitated by physical interactions between syntaxin $1 \mathrm{~A}$ and Gbetagamma. J Biol Chem 275, 6388-6394 (2000).

223. Shigemoto, R. et al. Differential presynaptic localization of metabotropic glutamate receptor subtypes in the rat hippocampus. J Neurosci 17, 7503-7522 (1997).

224. Huang, C.C., Yang, D.M., Lin, C.C. \& Kao, L.S. Involvement of Rab3A in vesicle priming during exocytosis: interaction with M unc13-1 and M unc18-1. Traffic 12, 1356-1370 (2011).

225. Han, S.Y., Park, D.Y., Park, S.D. \& Hong, S.H. Identification of Rab6 as an N-ethylmaleimidesensitive fusion protein-binding protein. Biochem J 352 Pt 1, 165-173 (2000).

226. Lobingier, B.T. \& Merz, A.J. Sec1/M unc18 protein Vps33 binds to SNARE domains and the quaternary SNARE complex. M ol Biol Cell 23, 4611-4622 (2012).

227. Chuang, W. \& Lagenaur, C.F. Central nervous system antigen P84 can serve as a substrate for neurite outgrowth. Dev Biol 137, 219-232 (1990).

228. Sano, S., Ohnishi, H., Omori, A., Hasegawa, J. \& Kubota, M. BIT, an immune antigen receptorlike molecule in the brain. FEBS Lett 411, 327-334 (1997).

229. Gitler, D. et al. Different presynaptic roles of synapsins at excitatory and inhibitory synapses. J Neurosci 24, 11368-11380 (2004).

230. Wagner, S.A. et al. Proteomic analyses reveal divergent ubiquitylation site patterns in murine tissues. Mol Cell Proteomics 11, 1578-1585 (2012).

231. Walzthoeni, T. et al. xTract: software for characterizing conformational changes of protein complexes by quantitative cross-linking mass spectrometry. Nat Methods 12, 1185-1190 (2015).

232. Chen, Z.A. et al. Structure of Complement $\mathrm{C} 3(\mathrm{H} 2 \mathrm{O})$ Revealed By Quantitative CrossLinking/M ass Spectrometry And M odeling. M ol Cell Proteomics 15, 2730-2743 (2016).

233. Guerrier, C. \& Holcman, D. The First $100 \mathrm{~nm}$ Inside the Pre-synaptic Terminal Where Calcium Diffusion Triggers Vesicular Release. Front Synaptic Neurosci 10, 23 (2018).

234. Kim, M.H., Korogod, N., Schneggenburger, R., Ho, W.K. \& Lee, S.H. Interplay between $\mathrm{Na}+\mathrm{Ca} 2+$ exchangers and mitochondria in $\mathrm{Ca} 2+$ clearance at the calyx of Held. J Neurosci 25, 6057-6065 (2005).

235. Zenisek, D. \& Matthews, G. The role of mitochondria in presynaptic calcium handling at a ribbon synapse. Neuron 25, 229-237 (2000).

236. Glancy, B. \& Balaban, R.S. Role of mitochondrial Ca2+in the regulation of cellular energetics. Biochemistry 51, 2959-2973 (2012).

237. Das, A.M \& \& Harris, D.A. Control of mitochondrial ATP synthase in heart cells: inactive to active transitions caused by beating or positive inotropic agents. Cardiovasc Res 24, 411-417 (1990). 
238. Ionescu, L. et al. Graded recruitment and inactivation of single InsP3 receptor Ca2+release channels: implications for quantal [corrected] Ca2+release. J Physiol 573, 645-662 (2006).

239. Corbett, E.F. et al. The conformation of calreticulin is influenced by the endoplasmic reticulum luminal environment. J Biol Chem 275, 27177-27185 (2000).

240. Sakono, M., Seko, A., Takeda, Y. \& Ito, Y. PDI family protein ERp29 forms 1:1 complex with lectin chaperone calreticulin. Biochem Biophys Res Commun 452, 27-31 (2014).

241. Baydyuk, M., Xu, J. \& Wu, L.G. The calyx of Held in the auditory system: Structure, function, and development. Hear Res 338, 22-31 (2016).

242. Beguin, P. et al. FXYD7 is a brain-specific regulator of Na,K-ATPase alpha 1-beta isozymes. EMBO J 21, 3264-3273 (2002).

243. Geppert, M. et al. Synaptotagmin I: a major $\mathrm{Ca} 2+$ sensor for transmitter release at a central synapse. Cell 79, 717-727 (1994).

244. Greenman, C. et al. Patterns of somatic mutation in human cancer genomes. Nature $\mathbf{4 4 6}, \mathbf{1 5 3 -}$ 158 (2007).

245. Hojjati, M.R. et al. Kinase activity is not required for alphaCaM KII-dependent presynaptic plasticity at CA3-CA1 synapses. Nat Neurosci 10, 1125-1127 (2007).

246. Tani, M., lida, H. \& Ito, M. O-glycosylation of mucin-like domain retains the neutral ceramidase on the plasma membranes as a type II integral membrane protein. J Biol Chem 278, 1052310530 (2003).

247. Rohrbough, J. et al. Ceramidase regulates synaptic vesicle exocytosis and trafficking. J Neurosci 24, 7789-7803 (2004).

248. Hering, H., Lin, C.C. \& Sheng, M. Lipid rafts in the maintenance of synapses, dendritic spines, and surface AM PA receptor stability. J Neurosci 23, 3262-3271 (2003).

249. Luo, L. Actin cytoskeleton regulation in neuronal morphogenesis and structural plasticity. Annu Rev Cell Dev Biol 18, 601-635 (2002).

250. Spence, E.F. \& Soderling, S.H. Actin Out: Regulation of the Synaptic Cytoskeleton. J Biol Chem 290, 28613-28622 (2015).

251. Clark, S.W. \& M eyer, D.I. Centractin is an actin homologue associated with the centrosome. Nature 359, 246-250 (1992).

252. Holleran, E.A., Tokito, M.K., Karki, S. \& Holzbaur, E.L. Centractin (ARP1) associates with spectrin revealing a potential mechanism to link dynactin to intracellular organelles. J Cell Biol 135, 1815-1829 (1996).

253. Yamagishi, N., Ishihara, K. \& Hatayama, T. Hsp105alpha suppresses Hsc70 chaperone activity by inhibiting Hsc70 ATPase activity. J Biol Chem 279, 41727-41733 (2004).

254. Gomes, A.F. \& Gozzo, F.C. Chemical cross-linking with a diazirine photoactivatable cross-linker investigated by M ALDI- and ESI-M S/MS. J M ass Spectrom 45, 892-899 (2010). 


\section{Acknowledgements}

I want to express my gratitude to everybody, who helped with the creation of this thesis and supported me on my rocky path to a doctoral degree.

First and foremost, I want to thank Prof. Dr. Henning Urlaub. I learned the craft of mass spectrometry in his lab years before I started my PhD and he was involved in sparking my enthusiasm for this method. I am grateful for the challenging project, given to a microbiologist, who had little clue about eukaryotes let alone mammals or brains in the beginning. Thank you Henning for your belief and excitement for this project, for letting me work quite independently, the possibility to go to numerous conferences and for cheering me up again, when I sometimes lost track of the bigger picture.

I would like to thank my committee members Prof. Dr. Reinhard Jahn and Prof. Dr. Silvio Rizzoli for their time and helpful discussions and suggestions in committee meetings and at scientific retreats in Ringberg and Teistungen. Furthermore, I am grateful to Dr. Juliane Liepe, Dr. Vlad Pena and Dr. Alexis Faesen for their agreement to be part of the extended thesis committee.

I am greatly indebted to Dr. Momchil Ninov for being my neurobiological Consigliere. He helped me multiple times over the years: He taught me how to prepare synaptosomes, prepared synaptosomes and checked their viability for me when the workflow grew to a continuous $20 \mathrm{~h}$ monster, performed all continuous sucrose density gradient centrifugations, gave me advice when I was cluelessly stumbling through tower $\mathrm{VI}$, and proof-read and commented $80 \%$ of this thesis. Momo, you are simply the best!

I am grateful to all the people, who provided me with biological samples, so I could focus on the mass spec side of sample preparation: Prof. Dr. Jörg Stülke for the Bacillus subtilis strain, Dr. Fabian Henneberg for the $20 \mathrm{~S}$ proteasome, and Dr. Sandra Schilbach for Pol II and TFIIH. I am especially thankful for Sandra's efforts to obtain on-bead assembled PIC CM ed TFIIH complexes, which we initially wanted to cross-link.

I want to thank the staff from the animal facility, especially Sascha Krause and Thomas Gundlach, for providing animals and for helping me with the sample harvest.

I am thankful to Dr. Ingo Wohlgemuth and Dr. Niels Fischer for helpful discussions regarding my weird bacterial ribosome cross-links. Ingo also provided an E. coli M G1655 strain that I used for in vivo crosslinking, but did not include in this thesis.

I am also grateful for the GGNB and the people, who fill it with life. It is providing the best support a $\mathrm{PhD}$ candidate can ask for.

I am grateful for everybody, who proof-read this thesis, corrected and improved its readability, i.e. Prof. Dr. Henning Urlaub, Dr. M aria Tauber, Dr. Alexandra Stützer, and Dr. M omchil Ninov. If the reader thinks that it is still hard to follow the flow of thoughts, he/she should read the initial Dostoevsky 'notes from the underground' version of this thesis with two sentences per page.

It is not possible to estimate the amount of help I have received from all members of the Bioanalytical mass spectrometry group over the years: Stimulating discussions in the downstairs office, at seminars, and in the hallway, and help with data analysis from Alexandra, Andreas, Juliane, Alexander, Olex, Aleks, Sofia, Ivan, Sunit, Kuan-Ting, and others; technical assistance especially from Monika Raabe, 
Uwe Plessmann, Annika Reinelt, Dr. Ralf Pflanz, Dr. Sabine König, and several student assistants. This group is awesome and I am very grateful for being a part of it.

I also want to thank everybody, who brought me back down to earth when the lab was about to swallow me, i.e. my climbing buddies Christian, Kuan-Ting, Flo and Caro, my fellow Biomolecules, my party buddy Andreas, and all other members of the mass spec group, who spent time with me outside of the lab.

At last, I am grateful to M aria, my partner in crime, for basically everything. 